Florida International University FIU Digital Commons

\title{
Carbon Nanotube- and Gold Nanoparticle-Based Materials For Electrochemical and Colorimetric Sensing Applications
}

Janak Paudyal 9255967

Florida International University, jpaud001@fiu.edu

DOI: 10.25148 /etd.FIDC001226

Follow this and additional works at: https://digitalcommons.fiu.edu/etd

Part of the Analytical Chemistry Commons, and the Materials Chemistry Commons

\section{Recommended Citation}

Paudyal, Janak 9255967, "Carbon Nanotube- and Gold Nanoparticle-Based Materials For Electrochemical and Colorimetric Sensing Applications" (2016). FIU Electronic Theses and Dissertations. 2996.

https://digitalcommons.fiu.edu/etd/2996 


\title{
FLORIDA INTERNATIONAL UNIVERSITY
}

\author{
Miami, Florida
}

\section{CARBON NANOTUBE- AND GOLD NANOPARTICLE-BASED MATERIALS FOR ELECTROCHEMICAL AND COLORIMETRIC SENSING APPLICATIONS}

\author{
A dissertation submitted in partial fulfillment of \\ the requirements for the degree of \\ DOCTOR OF PHILOSOPHY \\ in \\ CHEMISTRY \\ by \\ Janak Paudyal
}

2016 
To: Dean Michael R. Heithaus

College of Arts, Sciences and Education

This dissertation, written by Janak Paudyal, and entitled Carbon nanotube- and Gold Nanoparticle-based Composite Materials for Electrochemical and Colorimetric Sensing Applications, having been approved in respect to style and intellectual content, is referred to you for judgment.

We have read this dissertation and recommend that it be approved.

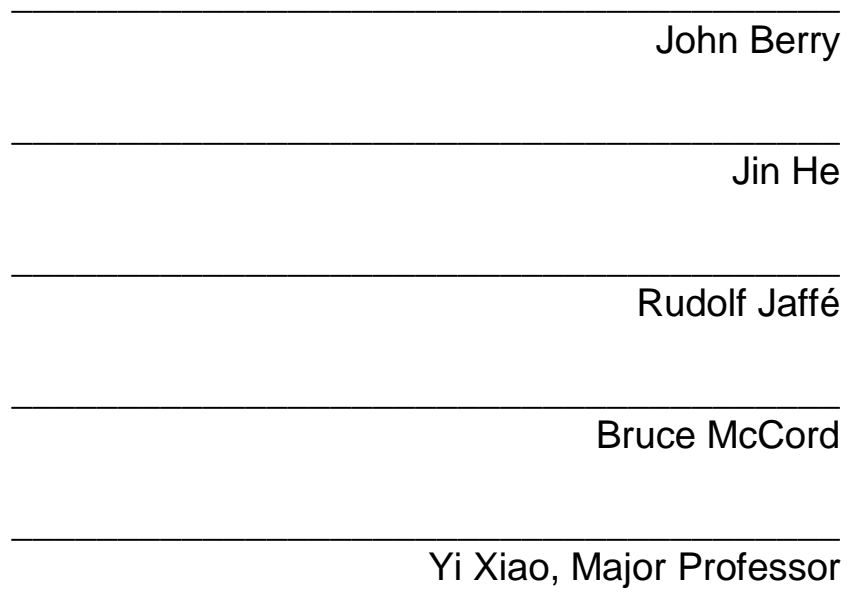

Date of Defense: November 9, 2016

The dissertation of Janak Paudyal is approved.

Dean Michael R. Heithaus

College of Arts, Sciences and Education

Andrés G. Gil

Vice President for Research and Economic Development and Dean of the University Graduate School

Florida International University, 2016 
(C) Copyright 2016 by Janak Paudyal

All rights reserved. 


\section{DEDICATION}

I dedicate this work to my spouse Ajeeta Bhattarai, son Sushant Paudyal, my parents, brothers, and sisters for their support and motivation throughout this work. 


\section{ACKNOWLEDGMENTS}

I would like to express my sincere appreciation to the individuals that help me directly or indirectly to accomplish this achievement.

At first, I am indebted to my major professor Dr. Yi Xiao; thank you for letting me work under your supervision. I would like to express my gratitude for constant encouragement, motivation, and support. Thank you for your tolerance and tireless efforts from the very beginning.

I very much possess to Dr. John Berry, Dr. Jin he, Dr. Rudolf Jaffe and Dr. Bruce McCord for serving as a committee member. I would like to thank for your valuable time, guidance and suggestions during last five years.

I would like to acknowledge Department of Chemistry and Biochemistry for the financial assistance; Graduate and professional student committee for travel funding and Florida International University for copyright permissions. I would like to thank Dr. Yong Cai and Wen bin Cui for letting me use ICPMS and DLS instruments and Dr. Wenzhi Li for AFM imaging; Thanks to all the faculty and staff members in Department of Chemistry and Biochemistry for the help.

I am grateful to all my lab mates for your helping hands both on and off the lab. Thank you very much Dr. Jianyuan Dai and Bhargav Guntupalli for assistance in my CNT project; Dr. Po Wang for PtNP characterization and Dr. Ping Ping Liang for AuNP experiments. Thank you again for wonderful memories.

At last, I thank all others who give me a helping hand during last five years but could not mention here personally. 


\title{
ABSTRACT OF THE DISSERTATION
}

\section{CARBON NANOTUBE- AND GOLD NANOPARTICLE-BASED MATERIALS FOR ELECTROCHEMICAL AND COLORIMETRIC SENSING APPLICATIONS}

by

\author{
Janak Paudyal
}

Florida International University, 2016

Miami, Florida

Professor Yi Xiao, Major Professor

Carbon nanotubes (CNTs) and gold nanoparticles (AuNPs) are widely used for sensing applications because of their distinctive electrical and optical properties, and we have explored the development of methods that enable the incorporation of these nanomaterials into new and improved sensing devices.

As a means for fabricating simple, low-cost and fast detection platforms for various applications, we have developed paper-based electrochemical detection platforms using CNTs or platinum nanoparticle (PtNP)-CNT composite materials. We describe the use of a paper-based, low density, three-dimensional thin film of interconnected CNTs as an electrode material. We studied the electrochemical properties of these paper-based CNT electrodes and demonstrated their use as an electrochemical sensor for the sensitive detection of guanine-based nucleotides. We further describe the functionalization of this paper-based electrode by fabricating a PtNP-SWCNT hybrid film via a vacuum filtrationbased method. The interconnected PtNP structure formed on top of the CNT-coated paper was directly used as an electrocatalyst for methanol oxidation. Compared to paper-based PtNP-SWCNT hybrid films formed by electrochemical deposition, hybrid films formed by vacuum filtration showed a higher electrochemical surface area and enhanced electrocatalytic response to methanol oxidation. 
We have also developed methods based around DNA-modified AuNPs, which offer an excellent colorimetric platform for target detection. The DNA density on the surface of modified AuNPs affects enzymatic activity, colloidal stability of AuNPs, the orientation of the probe DNA and its hybridization efficiency. The combination of all these factors ultimately dictates the reaction time and sensitivity of colorimetric assays. we demonstrate the use of dithiothreitol (DTT) as a modulator to control DNA surface coverage on the surface of AuNPs. Using this DTT treatment and a novel probe for exonuclease III activity, we have developed a colorimetric assay using DTT-treated, DNA-modified AuNPs that can achieve sensitive and rapid detection of DNA and enzymes relative to existing sensor platforms. 
Chapter 1. Introduction 1

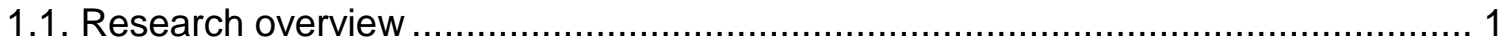

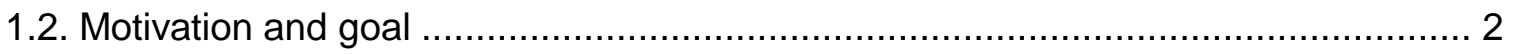

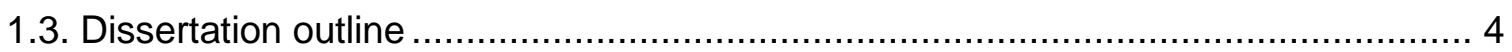

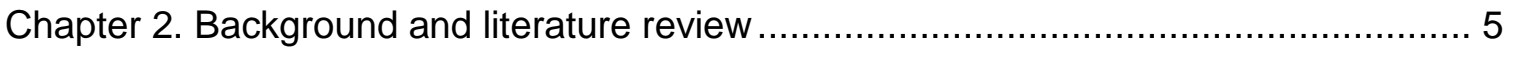

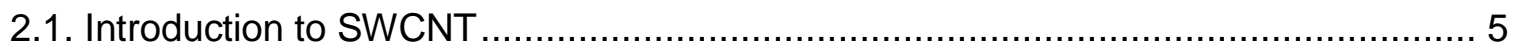

2.2. Electrochemical properties of SWCNT ....................................................... 6

2.3. Electrochemical properties of SWCNT ensembles ............................................ 6

2.4. Fabrication of CNT-based electrodes ........................................................ 8

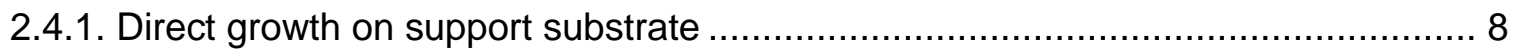

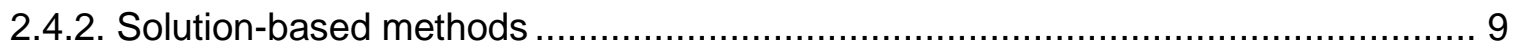

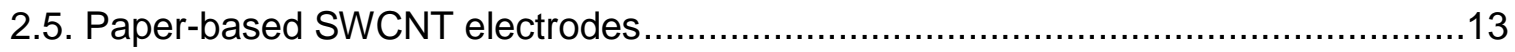

2.6. Paper-based Vacuum Filtered SWCNT (VF- SWCNT) thin film as a sensing

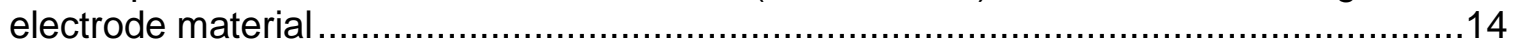

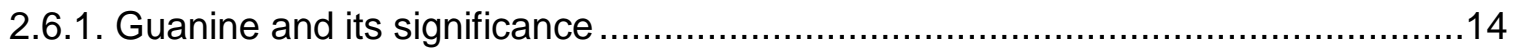

2.6.2. Current approaches to guanine detection ...................................................15

2.6.3. CNT-based electrochemical methods for guanine detection ..............................16

2.7. VF-SWCNT thin film as a conducting supporting material...................................18

2.8. SWCNT thin film as a conducting support for platinum .......................................19

2.8.1. Solution-based synthesis of Pt-CNT hybrid electrodes .....................................19

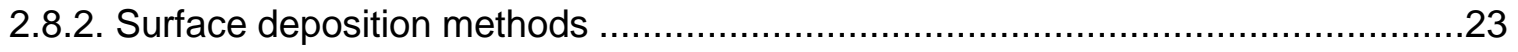

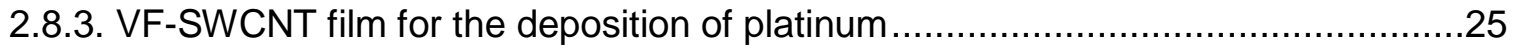

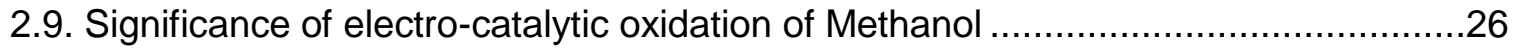

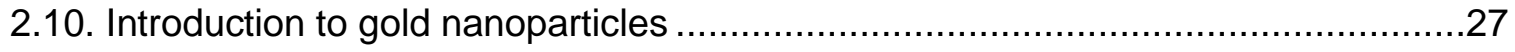

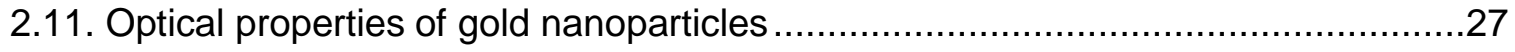

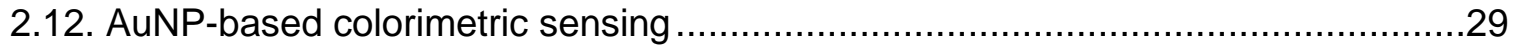

2.12.1. Functionalization of gold nanoparticles for enzyme activity …..........................29

2.13. DNA-functionalized AuNPs for colorimetric enzyme detection .............................30

2.14. DNA-AuNP as a substrate for exonuclease III ...................................................32 


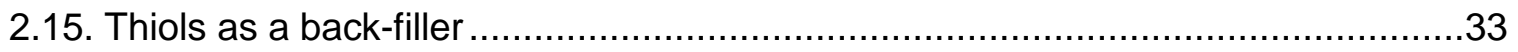

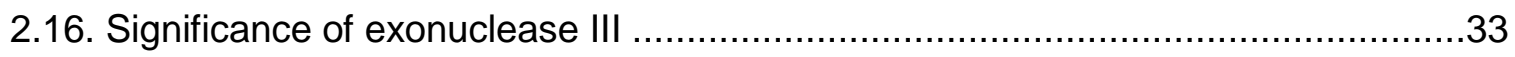

2.17. Current approaches to Exo III detection...................................................

Chapter 3. Paper-Based, Carbon Nanotube (CNT) Thin Film for Electrochemical

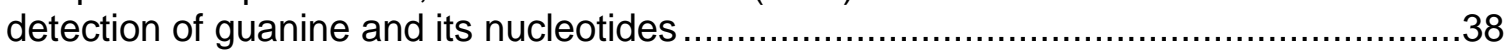

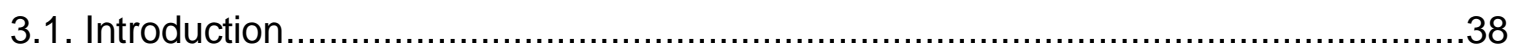

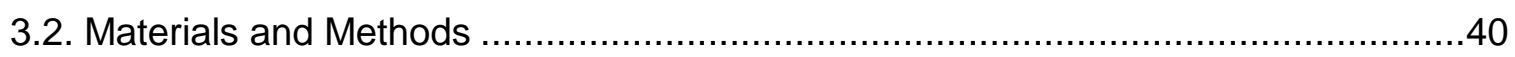

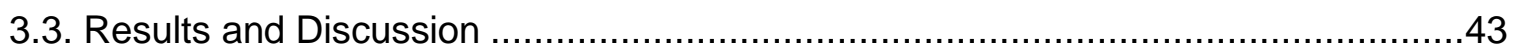

3.3.1. Fabrication of paper-based P-SWCNT thin film ..........................................43

3.3.2. Characterization of paper-based P-SWCNT thin films .....................................43

3.3.3. Fabrication of paper-based P-SWCNT film electrodes ...................................46

3.3.4. Electrochemical properties of paper-based P-SWCNT film electrode ..................48

3.3.5. Electrocatalytic response of paper-based SWCNT thin film electrode.................49

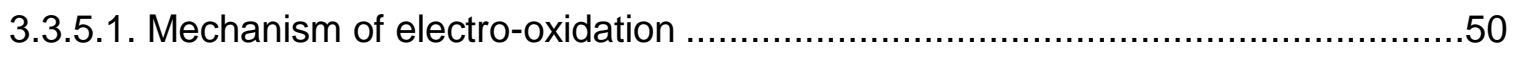

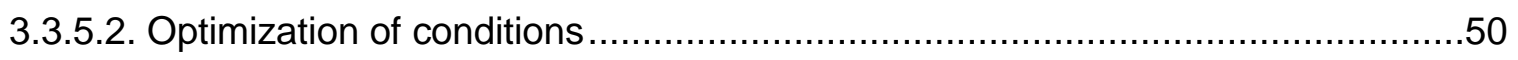

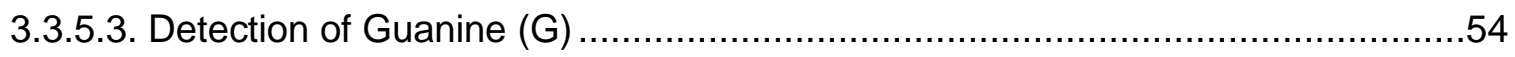

3.3.5.4. Detection of Guanosine monophosphate (GMP) .....................................55

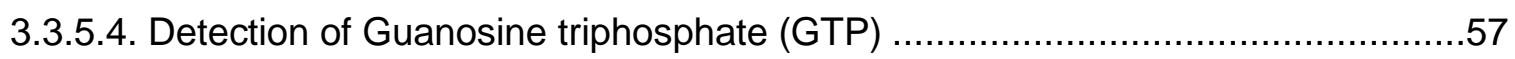

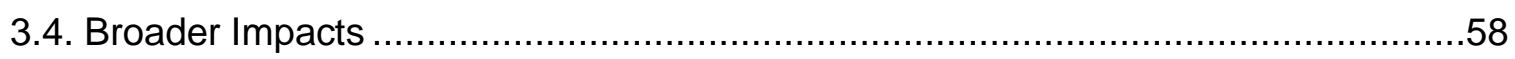

Chapter 4. A paper-based porous, three-dimensional Pt-CNT hybrid film electrode

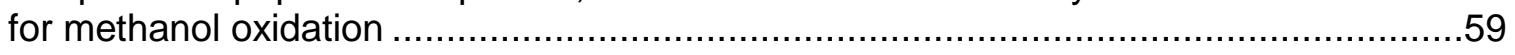

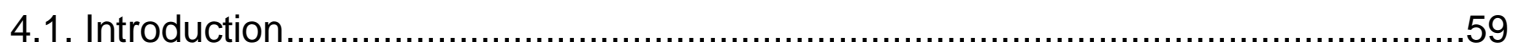

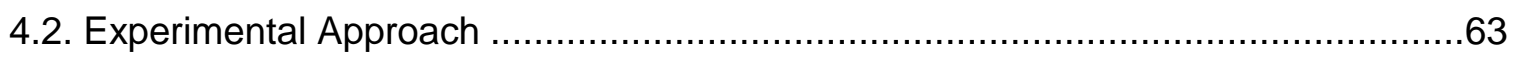

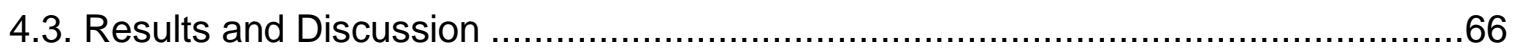

4.3.1. Fabrication of paper-based PtNP/SWCNT hybrid film by electrodeposition

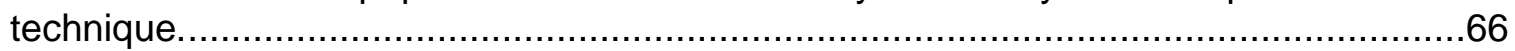

4.3.2. Characterization of the PtNP/SWCNT film prepared by electro-deposition. .........68

4.3.3. Evaluation of paper-based PtNP/SWCNT hybrid film electrode for electrochemical oxidation of methanol ............................................................ 71

4.3.4. Fabrication of paper-based PtNP/SWCNT hybrid film using vacuum filtration......73 4.3.5. Characterization of paper-based PtNP/SWCNT hybrid film prepared by vacuum filtration 
4.3.5.1. Pt concentration of the paper-based PtNP/SWCNT hybrid film .......................77

4.3.5.2. Morphology of PtNPs on the surface of PtNP/SWCNT hybrid film ..................78

4.3.5.3. State of Platinum on the surface of paper-based PtNP/SWCNT hybrid film ......79

4.3.6. Comparison of methanol oxidation of PtNP/SWCNT hybrid films .......................80

4.3.7. Stability and reproducibility of PtNP/SWCNT hybrid film .................................82

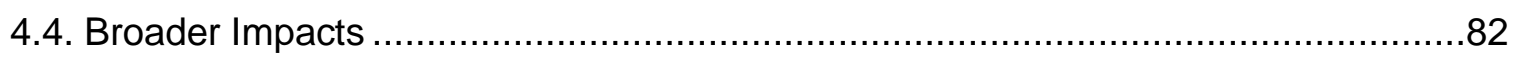

Chapter 5. A colorimetric assay for detecting DNA and exonuclease III based

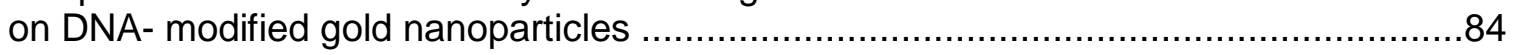

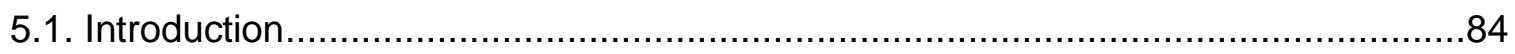

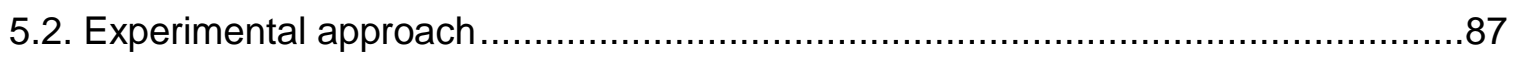

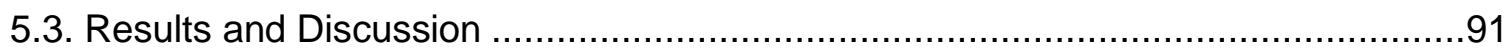

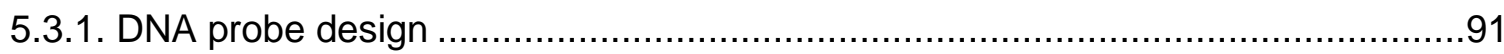

5.3.2. DNA modification on gold nanoparticles ...................................................93

5.3.3. Principle of colorimetric assay based on exonuclease III assisted target recycling for determining the activity of Exonuclease III ........................................96

5.3.4. DTT treatment of the DNA-AuNP probe.................................................98

5.3.4.1. DTT as a back-filler........................................................................ 98

5.3.4.2. Kinetics of DTT-displacement for DNA-modified AuNPs ................................100

5.3.4.3 Effect of DTT treatment on surface coverage of the DNA-modified AuNPs ......101

5.3.5. Activity of Exonuclease III on DTT-treated DNA-modified AuNPs ......................102

5.3.5.1. Effect of DTT treatment on the activity of Exonuclease III .............................102

5.3.5.2. Effect of DTT treatment on rate of Exo III catalyzed reaction .........................103

5.3.6. Effect of concentration of target DNA on Exo III-catalyzed reaction ...................104

5.3.7. Performance of newly designed DNA probe ..............................................106

5.3.8. Exonuclease assay based on DTT-treated DNA-modified AuNPs ...................108

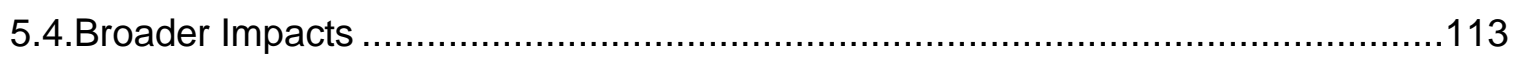

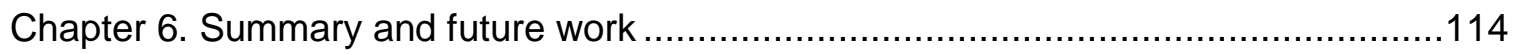

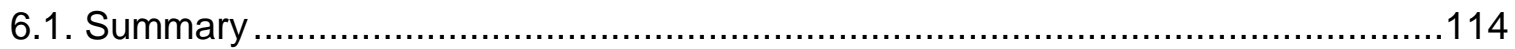

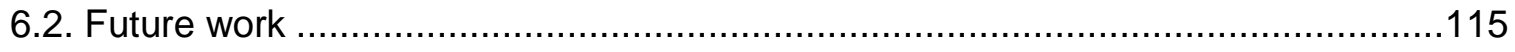

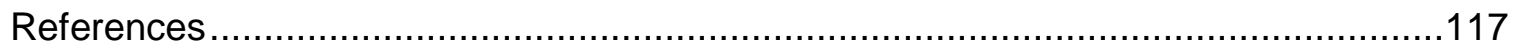

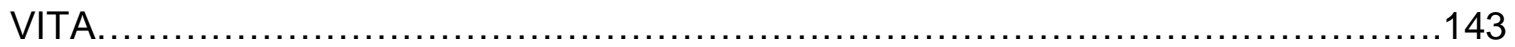




\section{LIST OF FIGURES}

FIGURE

PAGE

1. Diagram of the conceptual structure of CNTs formed from graphene. 5

2. SWCNT exhibit variable electrochemical properties based on the manner in which the graphene sheet is rolled up.

3. Chemical structure of guanine and its derivatives. 15

4. Schematic representation of oxidation of methanol on the surface of platinum. .26

5. Schematic representation of AuNP preparation through citrate reduction. .27

6. The shifting of color of mercury ion coated AuNPs from red to blue due to aggregation caused by target Lysin.

7. Cross-linked DNA-AuNPs as a colorimetric assay for Dnase I endonuclease enzyme.

8. Non-crosslinking-based mechanism for measuring DNase I enzyme activity with DNA-modified AuNPs in a colorimetric assay

9. X-ray crystal structure of exonuclease III from E. coli.

10. Hydrolysis of phosphodiester bonds at the $3^{\prime} \mathrm{OH}$ group by Exo III. .35

11. Fabrication of paper-based P-SWCNT thin film. (A) Schematic representation of vacuum filtration (VF) method. (B) photograph of paper-based P-SWCNT thin film.

12. Surface morphology of paper-based P-SWCNT thin films. Atomic force microscopy images of P-SWCNT thin films with different surface densities varying from $2.2 \mu \mathrm{gg} / \mathrm{cm}^{2}$ to $17.6 \mu \mathrm{gg} / \mathrm{cm}^{2}$. 
13. Characterization of thickness of paper based P-SWCNT thin film. (A) Height profile of P-SWCNT from AFM image. (B) Thickness of various P-SWCNT films calculated from height profile as a function of surface density.....

14. Characterization of sheet resistance of paper-based P-SWCNT thin film.

15. Fabrication of paper-based P-SWCNT electrode. Schematic representation of production of P-SWCNT film electrode from paper-based SWCNT thin film.......48

16. Electrochemical properties of paper-based P-SWCNT electrode. (A) Representative cyclic voltammetry of the paper-based P-SWCNT film electrode in potassium ferricyanide solution and $(\mathrm{B})$ variation of electron transfer rate as a function of surface density of SWCNT.

17. Electrochemical oxidation of guanine. Reaction mechanism, showing the formation of intermediates during the electrochemical oxidation of guanine. ......50

18. Effect of surface density of P-SWCNT film electrodes on electrochemical oxidation of guanine.

19. Effect of surface density on electrochemical oxidation of guanine nucleotides using the paper-based P-SWCNT film electrodes. (A) Variation of peak potential and $(B)$ signal to noise ratio as a function of surface density....

20. Effect of $\mathrm{pH}$ on electrochemical oxidation of guanine using paper-based $\mathrm{P}$ SWCNT film electrode. (A) Background-subtracted DPV of guanine oxidation using paper-based $\mathrm{P}$-SWCNT film electrode at different $\mathrm{pH}$ and (B) the variation of peak potential and peak current as a function of $\mathrm{pH}$.

21. Electrochemical detection of guanine on the paper-based P-SWCNT film electrode. (A) Background-subtracted DVP of various concentrations of guanine. (B) A plot of peak current against the guanine concentration.

22. Electrochemical detection of GMP using paper-based P-SWCNT film electrode. (A) Background-subtracted DVP of various concentrations of GMP. (B) A plot of peak current against the concentration showing linear range.

23. Electrochemical detection of GTP on the paper-based P-SWCNT film electrode. (A) Background-subtracted DVP of various concentrations of GTP. (B) A plot of peak current against the concentration of GTP showing a linear range 
24. Schematic representation of formation of paper-based SWCNT thin film and deposition of PtNPs on the SWCNT film by electrodeposition method.

25. Current transients of SWCNT paper electrode during electrodeposition of platinum in $2.0 \mathrm{mM} \mathrm{H}_{2} \mathrm{PtCl}_{6}$ containing $0.5 \mathrm{M} \mathrm{H}_{2} \mathrm{SO}_{4}$

26. Calibration curve of standard ${ }^{95} \mathrm{Pt}$ for the determination of the amount of electrodeposited Pt from the surface of paper-based SWCNT thin film electrode.

27. Morphology of various films. FE-SEM images of (A) Mixed cellulose ester filter paper (B) paper-based SWCNT thin films prepared by vacuum filtration method, (C) PtNPs on the SWCNT surface, and (D) size distribution of PtNPs for the paper-based PtNP/SWCNT film analyzed using imageJ software.

28. State of Pt on the surface of paper-based SWCNT thin film. (A) XPS spectra showing oxidation state of platinum and (B) XRD spectra showing the crystalline state of PtNPs on the surface of paper-based SWCNT film.

29. Evaluation of methanol oxidation on various electrodes. Cyclic voltammetry responses of (a) paper-based SWCNT film electrode, (b) commercially purchased Pt electrode, (c) PtNP-deposited GCE (d) PtNP-deposited FTO and (e) paper-based PtNP/SWCNT hybrid film electrode in $0.1 \mathrm{M} \mathrm{H}_{2} \mathrm{SO}_{4}$ containing $0.1 \mathrm{M}$ methanol at the scan rate of $50 \mathrm{mV} / \mathrm{s}$.

30. Electroactive surface area of different electrodes. Cyclic voltammetry responses of (a) commercially purchased Pt electrode, (b) PtNP-deposited GCE, (c) PtNP-deposited FTO, and (d) paper-based PtNP/SWCNT film paper in $0.5 \mathrm{M} \mathrm{H}_{2} \mathrm{SO}_{4}$ at the scan rate of $50 \mathrm{mVs}^{-1}$.

31. Schematic representation of the preparation of paper-based PtNP/SWCNT hybrid thin film by vacuum filtration technique.

32. Characterization of PtNPs by dynamic light scattering (DLS) experiments .75

33. Calibration curve of standard ${ }^{95} \mathrm{Pt}$ for the determination of the amount of Pt of synthesized PtNPs. 
34. Calibration curve of of standard ${ }^{95} \mathrm{Pt}$ for determination of the amount of $\mathrm{Pt}$ on the surface of paper-based PtNP/SWCNT thin film prepared by vacuum filtration technique.

35. Morphology of of PtNP on the surface of PtNP/SWCNT hybrid film prepared by vacuum filtration. (A) FE-SEM images of paper-based PtNP/SWCNT hybrid film. (B) Size distribution of PtNPs analyzed using an imageJ software...78

36. State of Pt on the surface of paper-based PtNP/SWCNT hybrid thin film prepared by vacuum filtration technique. (A) XPS spectra showing oxidation state of platinum and (B) XRD spectra showing the crystalline state of PtNP on the surface of SWCNT.

37. Comparison of electrocatalytic performance of PtNPs prepared by vacuum filtration and electrodeposition methods.

38. Stability and reproducibility of paper-based PtNP/SWCNT hybrid film prepared by vacuum filtration.

39. The design of DNA probes for determining the activity of Exonuclease III. (A) Traditional probe without an abasic site is the substrate for its 3' to 5' exonuclease activity. (B) New probe substrate incorporated with AP site.

40. Characterization of gold nanoparticles.

41. Schematic representation of DTT displacement fluorescence assay for the determination of the concentration of fluorescence labeled DNA on the AuNPs.

42. The calibration curve of the standard solution of $1 X$ _FAM probe in the range of 0 to $200 \mathrm{nM}$ for the calculation of DNA probe density on the surface of AuNPs.

43. Schematic representation of target recycling in colorimetric assay of exonuclease III.

44. DTT as a back-filler for controlling DNA surface coverage on the DNA-modified AuNPs.

45. Kinetics of displacement of FAM-labeled DNA from the surface of the gold nanoparticle by DTT. 
46. Effect of DTT treatment on the surface coverage of DNA on the surface of AuNP. (A) Calibration curve to determine the change in surface coverage with different concentration of DTT and (B) change in surface coverage as a function of DTT treatment.

47. Increase in specificity of exonuclease III by treating with different concentrations of DTT.

48. Kinetics of exonuclease III on DTT treated DNA-AuNP probes: $(A)$ kinetics of AuNP aggregation and (B) plot of rate of reaction vs DTT concentration

49. Effect of target concentration on the rate of exonuclease catalyzed reaction: The plot of the rate of aggregation (absorbance ratio) against the target concentration in the range of 0 to $200 \mathrm{nM}$.. .104

50. Effect of Effect of DTT treatment on target concentration on the rate of exonuclease catalyzed reaction: The plot of rate of aggregation (absorbance ratio) against the target concentration in the range of 0 to $250 \mathrm{nM}$ for $0,20,60 \mu \mathrm{M}$ DTT treated probe-AuNP substrates.

51. Comparison of performance of newly designed probe with the dual activity of exonuclease III (1X) with the traditional probe with 3' to 5' exonuclease activity (OX): the kinetics of exonuclease III reaction for $(\mathrm{A}) \mathrm{OX}$ and (B) $1 \mathrm{X}$ probe.

52. Schematic representation of DTT displacement fluorescence assay for the determination of the concentration of fluorescence labeled DNA on the surface of AuNP

53. Calibration curve of of DTT treated 1X_NoFAM probe in the range of 0 to $150 \mathrm{nM}$ for the determination of surface coverage by DTT displacement method

54. Effect of different concentration of $\mathrm{NaCl}(0,25,50$ and $100 \mathrm{mM})$ on the activity of Exonuclease III on DTT treated gold nanoparticle substrate in the presence (+) and the absence (-) of target DNA (A, B, C and D respectively). 
55. Effect of different concentration of $\mathrm{MgCl}_{2}(0,3,6$ and $9 \mathrm{mM})$ on the activity of Exonuclease III on DTT-treated probe-AuNP substrate in the presence (+) and in the absence (-) of target DNA (A, B, C and D respectively).

56. The plot of the ratio of absorbance against different concentration of exonuclease III in the range of 0 to 100 units $/ \mathrm{ml}$. The absorbance ratio was measured after 15 minutes of the reaction of different concentrations of exonuclease III with DTT-treated $1 \mathrm{X}$ probe DNA on the surface of the gold nanoparticle in the presence of $50 \mathrm{nM}$ of target DNA in $10 \mathrm{mM}$ Tris-acetate buffer $\mathrm{pH} 7.9$ containing $6 \mathrm{mM}$ of $\mathrm{MgCl}_{2}$. 


\section{LIST OF ABBREVIATIONS}

$\begin{array}{ll}\% & \text { Percentage } \\ \text { Abs } & \text { Absorbance } \\ \text { AFM } & \text { Atomic Force Microscope } \\ \text { Ag/AgCl } & \text { Silver/Silver Chloride } \\ \text { AP } & \text { Apurinic/ Apyridimic endonuclease } \\ \text { AuNP } & \text { Gold nanoparticle } \\ \text { CNT } & \text { Carbon nanotube } \\ \text { CV } & \text { Cyclic voltammetry } \\ \text { DI } & \text { deionized water } \\ \text { DLS } & \text { dynamic light scattering } \\ \text { DNA } & \text { deoxyribonucleic acid } \\ \text { DNA-AuNPs } & \text { DNA modified gold nanoparticles } \\ \text { DNase I } & \text { deoxyribonuclease I } \\ \text { DPV } & \text { Differential pulse voltammetry } \\ \text { DTT } & \text { Dithiothreitol } \\ \text { ECSA } & \text { electrochemical surface area } \\ \text { Exo III } & \text { Exonuclease III } \\ \text { FAM } & \text { Fluorescein } \\ \text { FTO } & \text { Fluorine-doped tin oxide } \\ \text { g } & \text { gram } \\ \text { G } & \text { Guanine } \\ \text { GCE } & \text { Glassy carbon electrode } \\ & \end{array}$




\begin{tabular}{|c|c|}
\hline GMP & Guanosine monophosphate \\
\hline GTP & Guanosine triphosphate \\
\hline $\mathrm{hr}$ & hour \\
\hline ICP & inductively coupled plasma \\
\hline L & litre \\
\hline $\mathrm{m}$ & milli \\
\hline M & moles per litre \\
\hline MCE & mixed cellulose ester \\
\hline $\mathrm{MCH}$ & 6-mercapto-1-hexanol \\
\hline $\min (s)$ & minute(s) \\
\hline MS & mass spectrometry \\
\hline MWCNT & Multi-walled carbon nanotubes \\
\hline $\mathrm{NADH}$ & dihydronicotinamide adenine dinucleotide \\
\hline $\mathrm{nM}$ & nano molar \\
\hline $\mathrm{nm}$ & nanometer \\
\hline P-SWCNT & Pure single-walled carbon nanotube \\
\hline PBS & Phosphate buffer saline \\
\hline PtNP & Platinum nanoparticle \\
\hline s & second \\
\hline SAM & Self-Assembled Monolayers \\
\hline SDS & Sodium dodecyl sulfate \\
\hline SEM & Scanning electron microscope \\
\hline SWCNT & Single-walled carbon nanotube \\
\hline Tris & Tris (hydroxymethyl)aminomethane \\
\hline UV-Vis & ultraviolet-visible \\
\hline
\end{tabular}




$\begin{array}{ll}\text { V } & \text { Volt } \\ \text { VF } & \text { Vacuum filtration } \\ \text { w/ } & \text { with } \\ \text { w/o } & \text { without } \\ \text { XPS } & \text { X-ray photoelectron spectroscopy } \\ \text { XRD } & \text { X-ray diffraction } \\ \beta & \text { beta } \\ \mu & \text { micro } \\ { }^{0} \mathrm{C} & \text { degree Celsius }\end{array}$




\section{Chapter 1. Introduction}

\subsection{Research overview}

Nanomaterials such as carbon nanotubes (CNTs), graphene, and gold nanoparticles (AuNPs) have been used in many detection platforms because of their distinctive sizeand shape-dependent physical, chemical, electronic and optical properties. These properties have led to improved selectivity, sensitivity, and rapid response compared to bulk material detection platforms. There is increasing interest in the fabrication of simple, cost-effective, portable, and disposable nanomaterial-based detection platforms for various sensing applications.

Carbon nanotubes have been used as both recognition elements and transducers for electrochemical sensing applications. Electrochemical detection itself offers a very simple, cheap, and portable platform for the detection of target analytes. To further simplify electrochemical detection, we describe a paper-based electrode material in which nanomaterials are loaded onto the paper substrate. Specifically, CNTs were used to fabricate paper-based electrodes with a simple vacuum filtration apparatus. This paper-based CNT electrode provides a flexible, versatile and disposable alternative to traditional electrodes. The use of these paper-based CNT electrodes for sensitive detection of guanine-based nucleotides is described in Chapter Three. These paperbased CNT electrodes were further functionalized with platinum nanoparticles (PtNPs) to form a paper-based PtNP-SWCNT hybrid film. Platinum nanoparticles assemble to form a continuous, porous, three-dimensional structure that is directly fabricated on top of a paper-based single-walled carbon nanotube (SWCNT) thin film through vacuum filtration assembly. The morphology, distribution and oxidation state of the PtNPs were studied in detail, and we have evaluated the performance of paper-based PtNP-SWCNT hybrid films using methanol as a model molecule in Chapter Four. 
Gold nanoparticles have been used as a colorimetric probe in combination with different recognition elements. Deoxyribonucleic acid-modified AuNPs have been most commonly used for target detection, with DNA acting as a recognition element and AuNPs acting as a colorimetric probe. The simplicity of the method lies in the fact that the presence of the target analyte can be detected by the naked eye without the need for instruments. However, this platform usually requires long detection times to achieve sensitive target detection. For fast and sensitive analysis, we have proposed new colorimetric assays for the detection of DNA and exonuclease III in Chapter Five. A DNA substrate that is targeted by both the 3'-to-5' exonuclease and AP endonuclease activity of exonuclease III was conjugated onto the surface of AuNPs. Dithiothreitol (DTT) was used as a diluent to modulate the surface coverage of DNA on the surface of AuNPs to obtain fast and sensitive detection of DNA and enzyme.

\subsection{Motivation and goal}

The overall aim was to fabricate simple, low-cost, portable and efficient nanomaterial-based platforms for electrochemical and colorimetric sensing applications. Carbon nanotubes have well-defined electrochemical properties and have been widely used for modification of traditional electrode surfaces to improve electron transfer rate and increase electrode surface area. On the basis of the excellent conductivity of CNTs, we have explored the feasibility of using them as an electrode material on a paper substrate. Paper-based electrochemical devices have proven to be a simple, cheap, portable and disposable alternative to traditional analytical methods for target detection. On the basis of the established electroactivity of CNTs for many analytes, the fabrication of paper-based CNT electrodes could be a valuable asset for next-generation sensing platforms. We have demonstrated the direct use of paper-based CNT electrodes as an electrochemical sensor, using guanine-based nucleotides as a model because of their 
significance in the mechanistic study of oxidative damage and various metabolic processes.

To further demonstrate the potential of paper-based CNT electrode, we functionalized these films with PtNPs to form paper-based PtNP-SWCNT hybrid films using two different methods: electrodeposition and vacuum filtration. Platinum was selected because of its excellent catalytic activity for hundreds of chemical reactions. We characterized the state of the Pt layer on the surface of the CNTs, and evaluated its performance using methanol as a model molecule.

Gold nanoparticles have been used as a simple, sensitive probe for colorimetric detection of target analytes as they generate a color change that is 1,000 -fold stronger than most strongly absorbing color dyes. The combination of AuNPs with DNA as a recognition element enables the naked-eye colorimetric detection of various analytes without the need for instruments. The difference in color of DNA-conjugated AuNPs (red) and aggregated AuNPs (purple or blue) arises from the removal of DNA from the surface of the gold nanoparticles as a consequence of direct or indirect interaction with the target. The DNA density on the surface of modified AuNPs is known to affect the activity of DNA-cleaving enzymes, as well as the colloidal stability of AuNPs and the orientation of the probe DNA and its hybridization efficiency with complementary targets. To achieve rapid and sensitive detection, we used DTT to adjust the coverage of thiol-conjugated DNA on the surface of the AuNPs. We used exonuclease III as a target to demonstrate the faster and sensitive detection achieved using DTT-treated AuNP-conjugated probes due to its biological significance as both an AP endonuclease and a 3'-to-5' exonuclease. 


\subsection{Dissertation outline}

Chapter Two presents a general literature review of the electrochemical properties of CNTs and the optical properties of gold nanoparticles. It also provides a complete state of the art literature review of the fabrication of CNT-based electrode materials for sensing applications, PtNP-CNT hybrid materials for catalysis applications, and DNA-modified AuNPs for colorimetric sensing applications. Furthermore, it discusses the importance of paper-based electrode materials. The significance and current methods of detection for three different targets-guanine-based nucleotides, methanol and exonuclease III-are also discussed. Chapter Three describes the fabrication, structural and electrochemical characterization of CNT-fabricated paper electrodes and their use for sensitive detection of guanine-based nucleotides. Chapter Four discusses the fabrication of PtNP-SWCNT hybrid material on a paper substrate, and its characterization and application for electrochemical methanol oxidation. Chapter Five describes the development of fast, sensitive colorimetric assays for DNA and enzyme detection based on DTT-treated, DNA-modified AuNPs. Chapter Six gives a summary of the dissertation and proposes future plans. 


\section{Chapter 2. Background and literature review}

\subsection{Introduction to SWCNT}

Carbon nanotubes (CNTs) are hollow, cylindrical allotropes of $\mathrm{sp}^{2}$-hybridized carbon atoms discovered in 1991 by lijima. ${ }^{1}$ Conceptually, CNTs can be represented as a cylinder obtained from folding a one-dimensional sheet of carbon atoms (graphene) as shown in figure 1. CNTs exhibit a high aspect ratio and surface area as a consequence of their long, hollow cylindrical structure. They are good conductors of heat and electricity and are chemically and thermally stable..$^{2,3}$
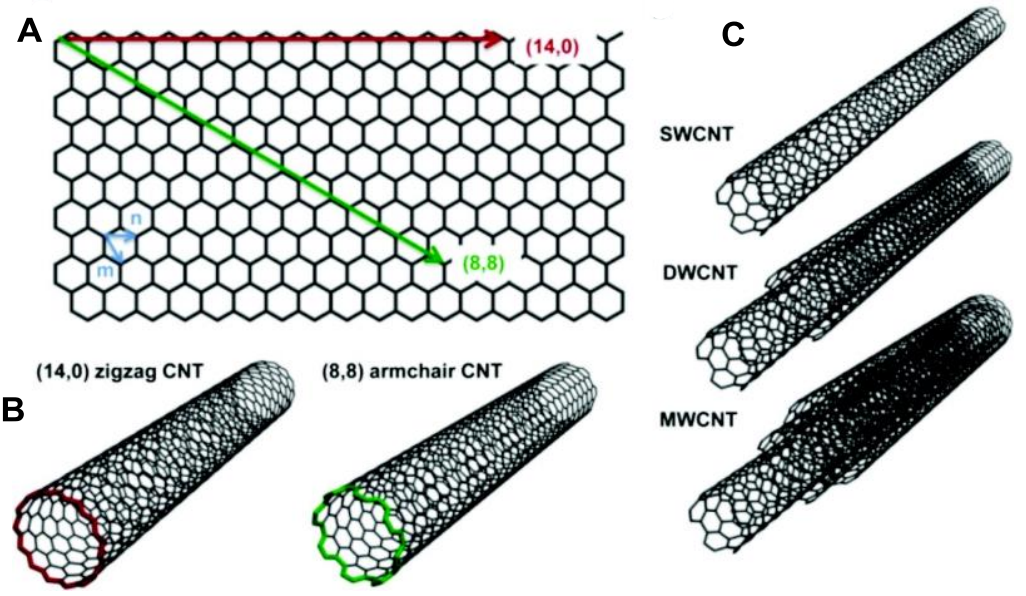

Figure 1. Diagram of the conceptual structure of CNTs formed from graphene. ${ }^{4}(A) A$ one-dimensional honeycomb-like graphene sheet can be folded in different manners to generate (B) electronically distinct CNTs, such as the zigzag CNT $(n=14, m=0)$ or the armchair CNT $(n=8, m=8)$. (C) single-walled (SWCNT), double-walled (DWCNT) and multi-walled (MWCNT) nanotubes.

Different ways of graphene sheet wrapping along the honeycomb lattice can be represented by its "roll up" vector na+mb, where, $\mathrm{n}$ and $\mathrm{m}$ are integers, and a and b are translational vector of graphene (Figure 1A). Different quantities of integers will yield CNTs with distinct electronic properties. For example, if $\mathrm{m}=0$, wrapping produces zigzag CNTs, whereas if $\mathrm{n}$ is equal to $\mathrm{m}$, it produces armchair nanotubes (Figure 1B). Considering the number of cylinders, CNTs may be single-walled (SWCNTs), double- 
walled (DWCNTs), or multi-walled (MWCNTs), as shown in figure 1C. The diameter of SWCNTs lies in the range of 1-2 nm whereas the diameter of MWCNTs lies in the range of $2-25 \mathrm{~nm}$, with lengths typically ranging from 0.2 to $5 \mu \mathrm{m}$. The high surface area-tovolume ratio, mechanical strength, chemical stability along with its unique electronic properties makes SWCNT especially attractive for electrochemical applications.

\subsection{Electrochemical properties of SWCNT}

Graphene is a zero-bandgap semiconductor because of its direction dependent conducting behavior. The low energy band structure of graphene can be represented by six Dirac cones (Figure 2B). The conducting states $\left(E_{f}\right)$ are present only at specific points along certain direction of momentum space but not in all directions as shown in figure $2 \mathrm{~B} .{ }^{5}$ On the basis of the direction of these momentum states with respect to the direction of conduction (chirality), rolling of graphene may produce either metallic or semiconductor CNT. ${ }^{3,6,7}$ If the rolling of graphene results in SWCNT with straight chiral vector, the CNT is called armchair and it will most likely be metallic in nature (Figure 2C).

Similarly, if graphene rolls with straight translational vector, the resulting CNT will be chiral or zigzag and it may act as a semiconductor (Figure 2D).

Unless separated, random samples of SWCNT contain a mixture of metallic and semiconducting SWCNTs, irrespective of the method of synthesis. Theoretical predictions have revealed that the typical ratio of semiconducting to metallic SWCNTs is approximately $3: 1$; therefore, SWCNT samples behave overall as a semiconducting nanomaterial. $^{8}$

\subsection{Electrochemical properties of SWCNT ensembles}

The purity, surface defects, size, and length of SWCNTs influence their electrochemical properties. Single-walled carbon nanotube ensembles are generally used as an electrode material, although there are reports of single SWCNTs being used 
as electrodes. The state of dispersion and their orientation on the electrode surface also greatly influence the electrochemical activities of SWCNTs.

A.

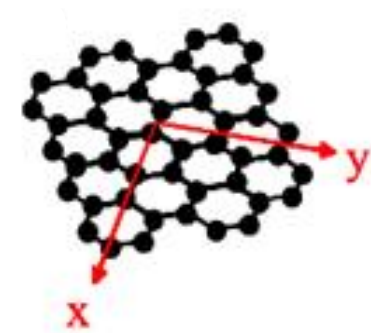

B.

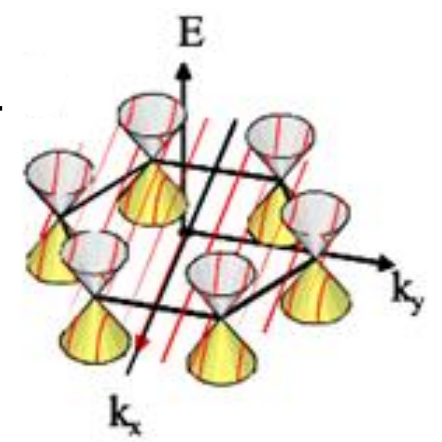

C.
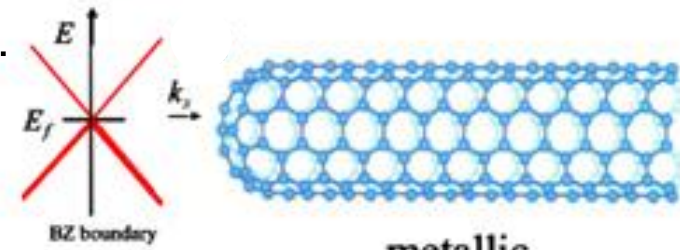

metallic

D.

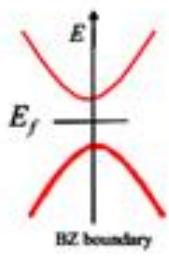

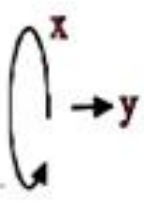

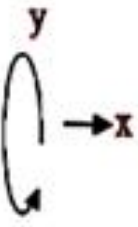

semiconducting

Figure 2. SWCNT exhibit variable electrochemical properties based on the manner in which the graphene sheet is rolled up. (A) The lattice structure of graphene, where the energy of conducting states varies as a function of the wave vector. (B) Six Dirac cones of graphene representing its low energy band structure. Graphene sheets can be rolled up to produce (C) metallic or (D) semiconducting SWCNTs. ${ }^{7}$

The tip and side wall of CNTs are respectively considered as the edge and basal plane of graphite..$^{9-11}$ Because of its edge plane-like structure, the tip of the CNT is believed to more electroactive compared to the side wall. Therefore, vertically-aligned CNTs are more electroactive compared to randomly-dispersed CNTs as a result of the edge plane-like structure.

The integrity of localized $\pi-\pi$ electrons is critical for maintaining CNT conductivity. ${ }^{2,12}$ The cleavage of a CNT's structure during purification or modification greatly reduces its 
conductivity. Conductivity is also diminished by carbonaceous and metallic surface impurities remaining after the growth process. Therefore, pure CNTs with intact structure are preferable for applications as an electrode material.

Carbon nanotubes are very inert, hydrophobic and insoluble in most solvents. ${ }^{13}$ They also have a tendency to form bundles due to $\pi-\pi$ interaction. The dispersed state of CNTs is another important criterion to conductivity, making it possible to effectively exploit their high surface area and minimize inter-tube junction resistance. Several methods have been used to construct SWCNT electrodes, with the aim of producing pure, highly dispersed, and minimally damaged CNTs for electrochemical applications.

\subsection{Fabrication of CNT-based electrodes}

Various methods have been used to develop CNT-based electrodes for different applications, such as electrochemical sensing,${ }^{14,15}$ electrocatalysis, ${ }^{16}$ super capacitors, ${ }^{17}$ lithium ion batteries, ${ }^{18}$ solar cells ${ }^{19}$ and fuel cells. ${ }^{20}$ Here, I focus on the fabrication of SWCNT-based electrodes for electrochemical sensing and electrocatalysis applications. The major challenge for those applications is to incorporate SWCNT into a working electrode without losing its pristine electrochemical properties. The electrochemical behavior of such CNT-based electrodes depends on their orientation, density, and spatial arrangement on the electrode substrate. However, the method of fabrication dictates both their orientation and the choice of the electrode substrate. In general, methods for the fabrication of CNT-based electrode can be defined in terms of direct growth on the substrate surface and solution-based methods.

\subsubsection{Direct growth on support substrate}

Direct growth of SWCNT on support substrates has been achieved by chemical vapor deposition (CVD) methods. The growth involves thermal decomposition of hydrocarbons in the presence of a metal catalyst at high temperature and pressure, ${ }^{21-23}$ 
For example, Viet et al. ${ }^{24}$ prepared a $\mathrm{Fe}_{2} \mathrm{O}_{3}$ catalyst by impregnating fumed alumina with $\mathrm{Fe}\left(\mathrm{NO}_{3}\right)_{3}$ dispersed in methanol solution for one hour. The silicon substrate was immersed in the catalyst solution for 10 minutes, heated at $130^{\circ} \mathrm{C}$, then transferred to a tube furnace and heated to $825^{\circ} \mathrm{C}$. Argon gas was passed through the tube, followed by ethanol as a carbon source at $1.25 \mathrm{~atm}$. Single-walled carbon nanotubes have been deposited successfully via similar methods on a variety of substrates, such as metal, ${ }^{25}$ carbon fiber, ${ }^{26}$ and carbon cloth. ${ }^{27}$

Single-walled carbon nanotube films obtained via CVD methods may be vertically aligned or entangled, depending on the distribution of catalyst on the surface of the substrate. ${ }^{28}$ Besides self- assembly ${ }^{14}$ and electrophoretic deposition, ${ }^{20}$ CVD is the only method to deposit vertically-aligned CNTs on a support substrate. The thickness of the film can also be controlled depending on the time of deposition. However, controlled deposition of catalyst on the substrate surface is still difficult and limited to certain substrates. For example, there is no straightforward method to deposit SWCNT films on the surface of traditional electrode substrates such as gold, glassy carbon (GC), and fluorine-doped tin oxide (FTO) for sensing and catalysis applications. Furthermore, the method is hard to scale to large areas and is not suitable for mass production, and the removal of carbonaceous and metal impurities is also difficult. Therefore, CVD is not preferred for fabrication of SWCNT-based electrodes for electrochemical sensing or electro-catalytic applications.

\subsubsection{Solution-based methods}

In order to prepare inexpensive CNT-based electrodes, SWCNT thin films can be prepared by depositing a suspension solution of pre-synthesized SWCNTs onto different substrates. Compared to methods that use direct growth on the substrate surface, solution-based methods do not require complex instrumentations, are compatible with 
various electrode substrates and only entail dispersion and deposition of SWCNTS on the substrate surface. The major challenges of these solution-based methods for the preparation of SWCNT electrodes include protecting the inherent electrochemical properties of the SWCNTs during fabrication, ensuring dispersion in a suitable solvent and subsequent removal of that solvent from the substrate, and promoting de-bundling of SWCNTs.

The methods of formation of SWCNT electrodes can be categorized into a variety of types, described in the following sections.

\subsubsection{SWCNT paste electrode}

Single-walled carbon nanotubes have been mixed with a binder to form a paste, which is then packed or sealed within a tube to make a SWCNT electrode. ${ }^{29,30}$ For example, Xuzhi et al. ${ }^{30}$ mixed SWCNTs with ionic liquid 1-butyl-3-methylimidazolium hexafluophosphate $\left(\mathrm{BMIMPF}_{6}\right)$ in a 1:1 ratio in a mortar and then packed the paste into a glass tube to make an electrode, with copper wire inserted inside the paste for the electrical connection. Such paste electrodes have been fabricated with other ionic liquids, ${ }^{30,31}$ paraffin oil, ${ }^{29}$ and mineral oil. ${ }^{32,33}$

\subsubsection{Deposition of SWCNT solution on conducting electrode surfaces}

Deposition of SWCNT on the electrode surface is the simplest method of electrode preparation, and there are several different versions of this approach.

In the 'dip coating' method, a support substrate is repeatedly immersed in a colloidal solution of SWCNTs using a dip coater. ${ }^{34-37}$ For example, Jang et al. ${ }^{34}$ prepared a colloidal solution of nitric acid-treated SWCNTs in 1,2-dichlorobenzene solution by sonicating for $10 \mathrm{hr}$. A glass substrate was prepared with a mixture of sulphuric acid and hydrogen peroxide to remove impurities and make it solventphilic, and then cleaned and dried before dipping into the colloidal solution. The step motor in the dip coater was 
mounted to allow the repeated movement of the platform containing the SWCNT solution upward and downward, while the glass substrate was held in place on the hanger. Dip coating has been used to prepare SWCNT films on glass, ${ }^{34,35}$ silicon oxide, ${ }^{36}$ and carbon fiber. ${ }^{37}$ However, dip coating results in a SWCNT layer on both sides of the support substrate, which is not desirable in all cases. The aggregation of SWCNTs is also hard to control, as shown in carbon fiber in common solvents. ${ }^{37}$ The use of more soluble surfactants than common solvents is limited by the requirement of extra steps to remove it. Moreover, surface adhesion is modest because of the inertness of SWCNT.

The 'drop casting' method involves dispersion of SWCNTs in a suitable solvent via ultra-sonication; the resulting solution is then dripped or pipetted onto the electrode surface, followed by the evaporation of the solvent. This seemingly simple process is complicated by the insolubility of non-functionalized SWCNTs in most solvents. Liu et al. ${ }^{38}$ have used solvents such as benzene, acetonitrile, acetone and sodium dodecyl sulfate (SDS) solution to disperse pristine SWCNTs prior to drop-casting on the surface of Pt and Au electrodes. Although these SWCNT films did not produce well-resolved voltammograms, they appear to be promising super-capacitors in an acetonitrile solution containing $0.1 \mathrm{M}$ tetra-n-butylammonium hexafluorophosphate $\left(\mathrm{TBAPF}_{6}\right)$. To improve the electro-catalytic behavior of drop-casted SWCNT thin films, Hongxia et al. ${ }^{39}$ prepared a SWCNT solution by ultrasonic agitation of carboxylic acid-functionalized SWCNTs in N$\mathrm{N}$ dimethylformamide (DMF) solution. The dispersed solution was directly cast onto the surface of the glassy carbon (GC) electrode. The solvent was then evaporated using an infrared lamp to prepare SWCNT-modified GC electrode. Compared to bare GC electrode, CNT-modified electrodes showed higher electrochemical surface area and electro-catalytic behavior toward the oxidation of dopamine, ascorbic acid, and epinephrine. Wang et al. ${ }^{40}$ prepared a cast SWCNT thin film by a similar method, with 
carboxylic acid-functionalized SWCNTs on the surface of GC electrodes. They found that the amount of cast SWCNT is critical, as too little casting solution did not produce observable voltammetric currents for the oxidation of $0.3 \mathrm{mg} \mathrm{ml}^{-1}$ natural DNA, while too much solution increased the background current so much that the redox peak became worse.

Although direct deposition methods are simple and economical, aggregation of CNTs is very common in these films. The resulting bundle-like morphology often produces high noise and low reproducibility. ${ }^{37,41}$ Moreover, the CNT layers prepared by such methods are usually not uniform, and contain densely-packed CNTs that decrease the total available surface area of CNTs. The use of solubility-enhancing surfactants is limited by the need to remove these chemicals as a result of their negative effects on electrochemical properties. Single-walled carbon nanotube dispersion can be enhanced by mixing with ionic liquids ${ }^{42-44}$ as a binder before casting on the electrode surface. Xiao et al. $^{42}$ showed superior performance of SWCNT-1-butyl-3-methylimidazolium $\left(\mathrm{BMIMPF}_{6}\right)$ paste-modified GC electrodes compared to conventional SWCNT-modified GC electrodes for the detection of xanthine. The superior performance was attributed to the interaction of $\mathrm{BMIMPF}_{6}$ with SWCNT, which forces heavily entangled SWCNT bundles to form finer bundles, increasing the effective area of the electrodes. Similarly, Zhang et $a l^{43}$ showed that the use of ionic liquid as a binder promoted entanglement of the SWCNTs to form cross-linking 3D networks that could not be achieved with other solvents such as dichlorobenzene, ethanol, and DMF. The three-dimensional structure of $\mathrm{SWCNT}-\mathrm{BMIMPF}_{6}$ composite materials greatly increased the effective electrode surface area. Casting methods have been used to fabricate SWCNT-based electrodes on the surface of $\mathrm{Au},{ }^{38} \mathrm{Pt}^{38} \mathrm{GC},{ }^{39,40}$ and graphite ${ }^{45,46}$ among other materials. 
Like drop-casting methods, 'spraying' methods also involve the sprinkling of a highlydispersed SWCNT solution onto the support substrate followed by solvent evaporation. For example, Kaempgen et al. ${ }^{17}$ suspended SWCNTs in pure water with a tip sonicator. The suspended solution was then sprayed onto a polyethylene-therephthalate (PET) substrate placed on a hot plate at $100^{\circ} \mathrm{C}$. The water evaporated quickly, leaving a very thin film of SWCNT on the PET surface. That film was used as a supercapacitor. Similarly, Kim et al. ${ }^{47}$ sprayed a SWCNT solution in dodecylbenzenesulfonate (SDBS) onto a glass substrate to chemically reduce Pt for solar cell applications. Sprayed SWCNT films have been produced on silicon, ${ }^{48}$ glass, ${ }^{47}$ and PET substrates. ${ }^{17}$

Finally, deposition can be achieved via 'vacuum filtration' methods. The vacuum filtration involves dispersion of SWCNTs in aqueous surfactant solutions; these are then trapped on the surface of a porous filter membrane through vacuum-induced flow, and the surfactant is washed away with copious amounts of solvent. Vacuum filtration forms thin, uniform and porous SWCNT films on porous substrates. The solvent used for dispersion can be washed away easily, in contrast to other methods where this step can pose a challenge.

The density of CNTs used strongly affects the characteristics of the resulting film. ${ }^{49,50}$ Low density (close to percolation density) thin films are preferable for sensing applications, as it reduces the background current. The use of low-density CNTs for electrochemical sensing is permissible when used to modify conducting electrode surfaces such as gold, platinum, or glassy carbon electrodes. However, this is not suitable for non-conducting surfaces such as paper.

\subsection{Paper-based SWCNT electrodes}

Paper is an abundant, inexpensive, biocompatible, disposable and environmentfriendly substrate that could enable the development of simple, low-cost, portable, 
quantitative sensors for the analysis of different target molecules. Paper has been used in the fabrication of paper-based analytical devices, ${ }^{51}$ colorimetric ${ }^{52}$ and electrochemical sensors, ${ }^{53}$ and electrodes. ${ }^{54}$ For electrochemical applications, the use of paper rather than traditional electrode materials such as GC, gold or graphite not only reduces the costs but also simplifies the fabrication process. The use of paper as a substrate for electrochemical applications requires the deposition of a conductive layer, and CNTs, graphene or nano-composites of those materials with metal nanoparticles or polymers are materials of choice because of their large surface area and conducting behavior. Several conducting nanomaterials such as $\mathrm{CNTs}^{55,56}$ and CNT-gold ${ }^{57}$ have been deposited onto the surface of the paper, and the resulting electrodes have been used directly for electrochemical applications. For example, Da Costa et al. ${ }^{56}$ used inkjet printing to print SDS-dispersed MWCNTs onto paper. We have chosen SWCNTs and SWCNT composites with metal nanoparticles $(\mathrm{Pt})$ as a conducting material, because of their utility for electro-catalysis and electrochemical sensors.

\subsection{Paper-based Vacuum Filtered SWCNT (VF- SWCNT) thin film as a sensing electrode material}

\subsubsection{Guanine and its significance}

We selected guanine and its derivatives as target molecules to demonstrate the use of paper-based VF-SWCNT thin film as an electrochemical sensor. Guanine is one of the four bases found in DNA and RNA and contains the fusion of pyridine and imidazole rings as shown as shown in figure 3A. Guanine is linked to pentose, a five-carbon sugar molecule, to form the guanosine nucleoside, as shown in Figure 3B. Guanosine can be phosphorylated via esterification at its 5' hydroxyl group to produce guanosine nucleotides such as guanosine monophosphate (GMP; Fig. 3C) and triphosphate (GTP; Fig. 3D). 
<smiles>Nc1nc2[nH]cnc2c(=O)[nH]1</smiles>

A. Guanine<smiles>Nc1nc2c(ncn2[C@@H]2O[C@H](CO)[C@@H](O)[C@H]2O)c(=O)[nH]1</smiles>

B. Guanosine<smiles>Nc1nc2c(ncn2[C@@H]2O[C@H](COP(=O)(O)O)[C@@H](O)[C@H]2O)c(=O)[nH]1</smiles>

C. Guanosine monophosphate<smiles>Nc1nc2c(ncn2[C@@H]2O[C@H](COP(=O)(O)OP(=O)(O)OP(=O)(O)O)[C@@H](O)[C@H]2O)c(=O)[nH]1</smiles>

D. Guanosine triphosphate

Figure 3. Chemical structure of guanine and its derivatives.

Besides nucleic acid synthesis, guanine-based molecules play important roles in intracellular signaling and as part of the energy reservoir in protein synthesis. ${ }^{58,59} \mathrm{An}$ abnormal change in guanine concentration is related to the deficiency of immunity system. Guanine concentration changes are also considered as indicative of certain diseases such as cancer ${ }^{60}$ and HIV infection. ${ }^{61}$ Guanine is also the most susceptible base to oxidative stress, ${ }^{45,59}$ and the detection of guanine levels in response to various compounds can be used to study mechanisms of oxidative stress.

\subsubsection{Current approaches to guanine detection}

Various methods have been developed to detect guanine and its derivatives. With the exception of a few techniques such as fluorescence ${ }^{62}$ or chromatography with an electrochemical detector, ${ }^{63}$ most methods rely on direct electrochemical detection at the 
electrode surface because of the relative ease with which guanine is oxidated as compared to other nucleobases. Direct electrochemical detection has several advantages-it is inexpensive, requires only a small sample volume and enables rapid analysis compared to other methods. Different electrodes materials such as nanocarbon, ${ }^{64}$ boron-doped diamond ${ }^{65}$ glassy carbon $^{66}$ or nanomaterials such as graphene, ${ }^{67,68}$ and CNT-modified electrodes have all been used for detection of guanine and its derivatives.

\subsubsection{CNT-based electrochemical methods for guanine detection}

The CNT-based electrodes have attracted enormous interest due to their excellent electrochemical properties. ${ }^{2,69}$ Various analytes such as $\mathrm{NADH},{ }^{70}$ hydrazine,$^{71}$ dopamine,$^{72}$ and uric acid, ${ }^{72}$ have been detected by electrodes modified with CNTs. Carbon nanotube-based electrodes are especially attractive for guanine detection because they can amplify the sensing signal produced when this molecule directly adsorbs onto the CNT surface via П-П interaction.

Pedano et al. ${ }^{33}$ prepared carbon nanotube paste electrodes by mixing MWCNT powder with mineral oil and then packing the paste firmly into a Teflon tube. The prepared electrode was used for the detection of guanine by chrono-potentiometric stripping analysis. Similarly, Balan et al. ${ }^{73}$ packed a Teflon tube with a paste of MWCNTs with cobalt pthalocyanine in mineral oil, and used the prepared electrode for the oxidation of guanine bases in single-stranded DNA using differential pulse voltammetry (DPV). Zhang et al..$^{30}$ prepared electrodes by mixing SWCNTs with an ionic liquid $\left(\mathrm{BMIMPF}_{6}\right)$ and packing the hand-mixed paste into a glass tube and wiring with copper. The electrode was then used for DNA detection using the oxidation signal from guanine.

In another format, the CNT solution was used to modify electrode surfaces such as graphite ${ }^{74} \mathrm{GC},{ }^{75}$ carbon paste, ${ }^{75}$ edge-plane pyrolytic graphite, ${ }^{45}$ and screen-printed 
carbon electrodes ${ }^{76}$ to enhance the electrochemical signal produced by nucleobases. The CNTs were dispersed in solvents including DMF, ${ }^{77}$ water, ${ }^{78}$ and dihexadecyl hydrogen phosphate. ${ }^{79}$ Wang et al. ${ }^{40}$ cast SWCNT-modified GC electrodes to detect DNA using the oxidation signal of guanine, using DPV and an accumulation time of five minutes. Similarly, Erdem et al. ${ }^{77}$ cast SWCNT dispersed in DMF onto the surface of GCE for direct oxidation of guanine using DPV to monitor nucleic acids and biomolecular interaction. The same group also prepared ${ }^{80}$ electrodes by casting MWCNT dispersed in DMF onto the surface of screen-printed graphite electrode for direct oxidation of guanine, using DPV to monitor DNA hybridization. Wang et al. ${ }^{75}$ prepared electrodes by casting carboxylic acid-functionalized MWCNT aqueous solution onto the surface of GCE. The prepared electrode was directly used for oxidation of guanine using cyclic voltammetry. The surface-confined MWCNTs were found to facilitate the adsorptive accumulation of guanine. Ye et al. ${ }^{81}$ also cast an aqueous solution of oxidized MWCNTs onto the surface of screen-printed carbon electrodes to prepare MWCNT-modified electrodes, which they used for direct oxidation of guanine and adenine in ssDNA and RNA via cyclic voltammetry. Erdem et al. ${ }^{74}$ demonstrated the use of MWCNT-modified graphite pencil electrodes for the detection of guanine by DPV. Increased porosity contributes to the superior performance of graphite pencil electrodes compared to commonly-used glassy carbon electrodes for the detection of guanine signal. Deng et al. ${ }^{78}$ cast an aqueous solution of boron-doped CNTs onto a GC surface for electrochemical detection of guanine and other bases.

To enhance electrochemical detection, CNTs has been combined with other chemicals such as ionic liquids, ${ }^{82-84}$ polymers, ${ }^{85}$ cyclodextrin,${ }^{86,87}$ gold ponanoparticles ${ }^{88}$ for the detection of guanine. For example, Abbaspour et al. ${ }^{76}$ casted oxidized-MWCNT on the surface of screen-printed carbon electrodes and further modified with cyclodextrin 
and polymer to prepare CNT-modified composite material for the detection of DNA. Similarly, Cai et al. $^{89}$ modified GC electrodes by casting carboxyl-functionalized MWCNTs on their surface. The functionalized MWCNTs were then used to immobilize $\mathrm{NH}_{2}$-modified probe DNA, and the difference in oxidation signal of guanine was used to detect the target DNA. Bollo et al..$^{90}$ casted O-MWCNTs dispersed in chitosan onto the surface of GCE for the detection of DNA using oxidation signal by DPV. Similarly, Liu et al. ${ }^{88}$ prepared polythionine/AuNP/MWCNT-modified GC electrodes by casting a mixture of MWCNTs and AuNPs followed by electro-polymerization of thionine for the detection of guanine using cyclic voltammetry. Wang et $a .^{83}$ modified GCE by casting DMFdispersed oxidized MWCNTs onto positively-charged GCE (due to electrodeposition of choline) for the sensitive detection of guanine along with other nucleotides.

\subsection{VF-SWCNT thin film as a conducting supporting material}

Single-walled carbon nanotube films prepared by vacuum filtration are a promising conducting support material for different catalysts as a result of several advantages over other such materials. Functionalization does not involve harsh treatment with chemicals, protecting the SWCNT structure, and the resulting porous structure both increases access to reactant material and facilitates removal of the products away from the catalyst surface. Additionally, the catalyst is located mainly on its external surface that is significant for the catalyst utilization efficiency. Finally, SWCNT thin films can act as both a conducting support as well as an electrode material, eliminating the need for extra electrode materials such as GC or gold or indium tin oxide (ITO) electrodes. To utilize these advantages, we deposited platinum nanoparticles (PtNPs) on the surface of SWCNT films to prepare a composite electrode material. The utility of VF SWCNT thin film as a platinum support was demonstrated by evaluating the performance of PtNP on its surface for the oxidation of methanol. 


\subsection{SWCNT thin film as a conducting support for platinum}

Because of their extraordinary electronic properties, SWCNTs have been used as a conducting support of metal catalysts to increase their efficiency. It is considered a nearideal conducting support for platinum catalysts, mainly because of its high surface area for PtNP dispersal, good electrical conductivity for faster heterogeneous electron transfer, and a smaller number of defects and edge-active atoms that prevent electrochemical corrosion. ${ }^{91,20,92}$ Much research is focused on Pt-SWCNT-based composite materials for sensing, ${ }^{92}$ fuel cell, ${ }^{4,5}$ and solar cell ${ }^{96}$ applications. The formation of CNT-metal nanoparticle composites was pioneered by Planeix et al., ${ }^{97}$ who deposited ruthenium on the surface of SWCNTs. The organic ruthenium salt was directly reduced by hydrogenation. Various methods have subsequently been used to deposit other metals, such as platinum, on the surface of SWCNTs. Those methods fall into several categories, described below.

\subsubsection{Solution-based synthesis of Pt-CNT hybrid electrodes}

In this approach, Pt-SWCNT composite material is first prepared in solution. The solution-based method is further discussed by categorizing it into following types.

\subsubsection{Impregnation and reduction of Pt precursor on CNT surfaces}

Impregnation is the most commonly used method of metal deposition. It involves covalent attachment of PtNPs onto the surface of functionalized CNTs by in situ reduction of a precursor salt. First, CNTs are functionalized with $-\mathrm{COOH}$ or $-\mathrm{OH}$ groups by chemical oxidation or dispersion with ionic liquids, ${ }^{98}$ surfactants (SDS solution, ${ }^{99}$ PSMA), ${ }^{100}$ polymers (polyvinylpyrrolidone (PVP)), ${ }^{101}$ or other compounds (e.g., 1aminopyrene, ${ }^{102}$ perylene tetracarboxylic acid). ${ }^{103}$ The functionalized CNTs are then dispersed in a suitable solvent-such as glycerol, ${ }^{104}$ ethylene glycol, ${ }^{105}$ a mixture of ethylene glycol and acetone, ${ }^{106}$ or 1 -propanol ${ }^{95}$ — containing precursor salt and reducing 
agents in the presence or absence of stabilizing agents (sodium citrate) or other chemicals required for the reduction of precursor salt. The reduction is usually carried out at $100-150{ }^{\circ} \mathrm{C}$ or with the aid of microwaves, using a suitable reducing agent such as ethylene glycol, ${ }^{106-108}$ formaldehyde, ${ }^{109,110}$ sodium citrate, ${ }^{111}$ citric acid, ${ }^{111}$ glycerol, ${ }^{104}$ or sodium borohydride. ${ }^{100,112}$ The resulting Pt-CNT composite is then purified by multiple washing and centrifugation cycles. The mixture is then washed, dried, and mixed with $5 \%$ Nafion solution before casting. In a typical in situ reduction, Selvaraj et al. ${ }^{104}$ prepared Pt-SWCNT composite material by using glycerol as a reducing agent. The acid-oxidized SWCNT was mixed with aqueous glycerol solution. Pt precursor salt dissolved in aqueous solution was slowly added into the SWCNT solution. The mixture was then heated to reflux at $130-140^{\circ} \mathrm{C}$ for $12 \mathrm{hrs}$ to reduce the precursor salt on the surface of SWCNT, then washed with water and dried at $60-70^{\circ} \mathrm{C}$ under vacuum, and finally mixed with $5 \%$ Nafion solution before casting on the surface of the graphite electrode.

The impregnation method suffers from the poor dispersion of pristine CNTs in most solvents and insufficient binding sites for anchoring PtNPs and precursor salt due to the inertness of pristine CNTs. Therefore, CNTs are usually functionalized. The most common method of functionalization is to create $-\mathrm{COOH}$ groups by chemical oxidation with nitric acid. ${ }^{95,107,109,110}$ The oxidation entails refluxing CNTs in concentrated acid for few hours. The harsh procedure usually damages the CNT structure, leading to the loss of conductivity and corrosion resistance. Carbon nanotube damage can be mitigated with less destructive functionalization techniques such as dispersion in ionic liquid ${ }^{98}$ or surfactants $\left(\right.$ SDS solution, ${ }^{99}$ PSMA), ${ }^{100}$ wrapping with polymers such as polyvinylpyrrolidone (PVP), ${ }^{101}$ or through hydrophobic interaction or dispersion in 1- 
aminopyrene $^{102}$ or $3,4,9,10$ perylene tetracarboxylic acid $^{103}$ through $\pi-\pi$ interaction. Noncovalent functionalization creates different functional groups that not only disperse SWCNTs in solution but also act as anchoring sites for the binding of precursor salts and PtNPs, leading to formation of more dispersed and uniform PtNPs compared to nonfunctionalized SWCNTs. Protecting agents such as $\mathrm{Na}$ citrate ${ }^{106}$ or PVP ${ }^{105}$ have also been used in order to prevent PtNP aggregation during the reduction of precursor salts.

\subsubsection{Colloidal synthesis of PtNP and incorporation with CNTs}

Colloidal methods are another solution-based approach, in which PtNPs are synthesized separately by reducing the precursor salt in the presence of stabilizing agents or suitable solvents to form citrate-capped ${ }^{92}$ or triphenylphosphine $\left(\mathrm{PPh}_{3}\right)^{-}$ modified $^{113}$ or uncapped in ethylene glycol $^{91,114}$ PtNPs. The pre-synthesized PtNPs are then attached onto the surface of CNTs by mechanical mixing, or $\mathrm{pH}$-induced adsorption, or by using linkers or polymers or ionic liquids to form PtNP-SWCNT composite material. The composite material is then mixed with $5 \%$ Nafion solution before casting onto the surface of graphite electrodes.

Besides the need for functionalization, the other problem associated with the colloidal synthesis approach is the presence of organic stabilizers, or coating agents present on the surface of PtNPs during colloidal synthesis. Stabilizing agents are essential for colloidal synthesis of PtNPs in solution during reduction of Pt salt; once the Pt is deposited on the surface of the CNT electrode, they should be removed, as they tend to block the access of reactants to the catalytic surface of the platinum and may alter the physiochemical properties of nanoparticles and thereby change their catalytic efficiency. Colloidal PtNPs have been synthesized with various stabilizing agents such as citrate,${ }^{115}$ polymer, ${ }^{116}$ amine,${ }^{117}$ proline, ${ }^{118}$ polyvinyl pyridine (PVP). ${ }^{119}$ so far citrate 
coated, ${ }^{115}$ PVP coated, ${ }^{119}$ or uncoated but ethylene glycol dispersed PtNPs ${ }^{120}$ have been incorporated on the surface of the CNT. Those capping agents or solvents are removed after incorporation with the $\mathrm{CNT} .{ }^{120}$ Alternatively, the pre-formed PtNPs have been modified with triphenylphosphine $\left(\mathrm{PPh}_{3}\right)^{113}$ for linking CNT.

For example, Hrapovic et al. ${ }^{92}$ prepared citrate-capped PtNPs with a diameter of 2.7 $\pm 0.7 \mathrm{~nm}$ by reducing an aqueous solution of a chloroplatinic acid at $80^{\circ} \mathrm{C}$ using sodium citrate as both a reducing agent and a capping agent. The charge interaction between citrate-capped PtNPs and negatively-charged, Nafion-solubilized SWCNTs was then used to incorporate PtNPs onto the surface of SWCNTs. Mu et al. ${ }^{113}$ prepared PtNPs with a diameter of $1.9 \pm 0.5 \mathrm{~nm}$ by reducing an ethylene glycol solution of chloroplatinic acid in alkaline medium $(\mathrm{pH} 12)$ by refluxing at $160^{\circ} \mathrm{C}$ for three hours. Ethylene glycol acts as both a reducing agent and protection solvent to prevent aggregation of PtNPs. The PtNP solution was dissolved in ethanol and mixed with a toluene solution of triphenylphosphine $\left(\mathrm{PPh}_{3}\right)$ with stirring to functionalize the PtNPs. The $\mathrm{PPh}_{3}$-modified PtNPs were then physically separated from the aqueous layer and washed with deionized water three times before incorporation with CNTs. The $\mathrm{PPh}_{3}$ modified PtNP solution was then mixed with CNT solution in toluene and ultra-sonicated for five days to form Pt-CNT composite material. Triphenylphosphine acts as a linker to attach PtNPs to SWCNTs as well as a protecting layer against PtNP aggregation. Anusorn et al. ${ }^{114}$ prepared PtNPs in ethylene glycol solution at $\mathrm{pH} 12.5$ by refluxing at $160{ }^{\circ} \mathrm{C}$ for three hours, and then mixed the colloidal PtNPs (diameter $=2-3 \mathrm{~nm}$ ) in ethylene glycol with either poly(sodium 4-styrenesulphonate) or oxidized SWCNTs in water. The $\mathrm{pH}$ of the solution was brought to $\mathrm{pH} 4.0$ to attach PtNP to the SWCNTs, after which the SWCNTs were filtered and dried. Zhang et al..$^{91}$ prepared PtNPs in ethylene glycol solution at $\mathrm{pH}$ 
13 by refluxing at $130^{\circ} \mathrm{C}$ for $3 \mathrm{hrs}$. Colloidal PtNPs (diameter $=2-3 \mathrm{~nm}$ ) in ethylene glycol were adsorbed onto the surface of CNT. Carbon nanotube film prepared by the CVD method was immersed into the PtNP solution in ethylene glycol for 24 hrs at room temperature, after which the excess residue of PtNP and solvent were washed away with ethanol solution. The Pt-adsorbed CNT film was then spun to form a yarnlike PtNPCNT electrode material. For better attachment of $\mathrm{Pt}$, the yarn was annealed at $600^{\circ} \mathrm{C}$ for 30 minutes in a nitrogen atmosphere.

\subsubsection{Surface deposition methods}

Surface deposition methods offer an alternative to solution-based methods, where PtNPs are deposited on SWCNT thin films by electrochemical reduction ${ }^{121-124}$ or electrodeless reduction ${ }^{125,126}$ of precursor salt. These surface deposition methods result in the deposition of PtNPs on the most accessible external surface of the CNT electrode.

\subsubsection{Electrochemical deposition method}

Electrochemical deposition is a very simple and inexpensive approach for the deposition of PtNPs on the surface of pre-formed CNTs. First, CNT thin films are

prepared by different methods such as direct growth on $\mathrm{SiO}_{2}{ }^{124,127}$ or carbon cloth, ${ }^{127,128}$ electrophoretic deposition of functionalized SWCNTs on indium tin oxide, ${ }^{20}$ vacuum filtration of CNT suspensions on the paper substrate followed by subsequent transfer to $\mathrm{GC}^{129,130}$ and carbon fiber ${ }^{129,130}$ or casting of MWCNTs in $\mathrm{GC}^{122}$ onto different electrode substrates. The prepared CNT film is then modified to incorporate the PtNPs and overcome their inherent inertness. Electrochemical oxidation of CNTs, ${ }^{122}$ casting of prefunctionalized CNTs, ${ }^{123}$ dispersion of precursor salt in an organic solvent (e.g., ethanol), ${ }^{121}$ and hydrophilic treatment of CNT films with sulphuric acid ${ }^{127,128}$ have all been used to increase the binding of PtNPs to CNTs. Metal precursors are then adsorbed 
onto the surface of the SWCNT film and electrochemically reduced, produces PtNPs of high purity, which adhere to the surface via covalent bonds. Platinum nanoparticle size and morphology can be controlled by varying deposition time, the concentration of precursor salt and applied potential.

Very thick films of CNT prepared by vacuum filtration have also been used for electrochemical deposition of platinum after transferring into suitable electrode material. For example, Sieben et al. ${ }^{129}$ prepared films from both pristine and oxidized SWCNT suspensions in different liquid media by vacuum filtration onto $0.1 \mu \mathrm{m}$ Omnipore membranes. The membrane was then removed to isolate the resulting SWCNT bucky paper, which was then stuck onto a polished GC electrode using 5\% Nafion solution as a binder. Platinum-ruthenium biocatalyst was then deposited on its surface by pulse potentiometry. The platinum particle size, distribution and degree of agglomeration were highly dependent on the dispersant used during vacuum filtration. The dependency is because of the higher electrochemical surface area of dispersed CNT as compared to its agglomeration. Zhu et al. ${ }^{130}$ prepared a hybrid film by vacuum filtration of a mixture of SWCNTs and carbon nanofibers (CNFs) in $1 \%$ Triton on $0.45 \mu \mathrm{m}$ nylon membranes. The surfactant was washed away with an isopropanol solution. The membrane was then peeled away, and the isolated bucky paper was attached to carbon fiber paper as a current collector with a homemade sample holder. Platinum nanoparticles were then deposited by pulse potentiometry. The use of CNF to increase the pore size $(>10 \mathrm{~nm})$ greatly increased the Pt utilization efficiency. However, the Pt was not uniformly distributed, and electrochemical surface area (ECSA) could not be further improved because of the relatively large Pt particles $-6 \mathrm{~nm}$ diameter versus $\sim 2 \mathrm{~nm}$ in commercial Pt/C catalysts. However, the hybrid film showed higher electrochemical durability 
compared to commercial Pt/C electrode. The same group ${ }^{131}$ further studied the durability of Pt on the surface of a hybrid bucky paper in which only $43 \%$ of ECSA was lost, compared to $80 \%$ of ECSA for a Pt/C electrode. They concluded that the main reason for the loss of ECSA on commercial Pt catalysts is carbon corrosion. Compared to commercial Pt/C catalyst, the bucky paper exhibited a two-fold slower rate of corrosion.

\subsubsection{Electrodeless deposition}

In this method, a Pt precursor is directly reduced on the surface of SWCNTs by the galvanic reaction, because of the higher reduction potential of the metal precursor $\left(+0.775 \mathrm{~V}\right.$ for $\left.\mathrm{PtCl}^{4} / \mathrm{Pt}\right)$ compared to SWCNTs $(0.5 \mathrm{~V})$ without the use of a catalyst or

reducing agent. Choi et al. ${ }^{125}$ deposited PtNPs directly on the surface of SWCNTs grown on the surface of silicon oxide just by dipping the SWCNT film into a solution of $\mathrm{Na}_{2} \mathrm{PtCl}_{4}$.

\subsubsection{VF-SWCNT film for the deposition of platinum}

The VF-SWCNT films offer a promising conducting support for $\mathrm{Pt}$ for several reasons. First, it has a unique porous nano-structured network of SWCNTs that maximizes the three-phase boundary of the electrode, conducting support and the Pt catalyst. Second, the high porosity of bucky paper maximizes the mass transfer process within the catalyst layer that maximizes the Pt utilization. Finally, PtNPs can be applied to the most accessible external surface of the bucky paper.

Up to now, platinum has been used in bucky papers by electro-deposition methods. These methods involve the formation of SWCNT electrode material by transferring the bucky paper onto current collectors such as GC or carbon paper, followed by reduction of Pt precursor directly onto the surface of the electrode. Until now, there have been no alternative deposition methods that directly maximize the surface area to volume ratio. 
Various reports have shown that this ratio can be maximized either by depositing welldispersed, small Pt particles or by forming continuous porous structures. To achieve this, we use a very thin VF-SWCNT film as a porous support for the deposition of pre-formed PtNPs. This also facilitates the removal of the aqueous phase from PtNP solution by vacuum-assisted (VA) layer-by-layer (LBL) assembly.

\subsection{Significance of electro-catalytic oxidation of Methanol}

The electro-catalytic efficiency of Pt can be evaluated using different molecules such as hydrogen peroxide, ${ }^{132,133}$ methanol, ${ }^{113,134,135}$ and formic acid. ${ }^{104,136}$ We selected methanol as an electrolyte because of its potential utility for portable fuel cells. Methanol oxidation reactions (MOR) at the anode of fuel cells are catalyzed by Pt. We, therefore, evaluated the performance of paper-based SWCNT thin films as a Pt support for electrodes for the anodic reaction of methanol. The oxidation reaction follows a threestep mechanism (Figure 4). ${ }^{137-139}$ Methanol is first adsorbed onto the surface of the Pt. The adsorbed methanol then undergoes dehydrogenation due to dissociation of the $\mathrm{C}-\mathrm{H}$ bond, forming $\mathrm{CO}$ as an intermediate. $\mathrm{CO}$ is then oxidized with the help of oxygencontaining compounds, such as water.
1. $\mathrm{CH}_{3} \mathrm{OH}$
$\longrightarrow\left(\mathrm{CH}_{3} \mathrm{OH}\right)_{\mathrm{ads}}$
Methanol adsorption
2. $\left(\mathrm{CH}_{3} \mathrm{OH}\right)_{\text {ads }}$
$\longrightarrow \mathrm{CO}_{2}+4 \mathrm{H}^{+}+4 \mathrm{e}^{-}$
$\mathrm{C}-\mathrm{H}$ bond formation
3.

$\begin{array}{lll}(\mathrm{CO})_{\text {ads }}+\mathrm{H}_{2} \mathrm{O} \longrightarrow \mathrm{CO}_{2}+2 \mathrm{H}^{+}+2 \mathrm{e}^{-} & \text {CO oxidation } \\ \mathrm{CH}_{3} \mathrm{OH}+\mathrm{H}_{2} \mathrm{O} \longrightarrow \mathrm{CO}_{2}+6 \mathrm{H}^{+}+6 \mathrm{e}^{-} & \text {Overall reaction }\end{array}$

Figure 4. Schematic representation of oxidation of methanol on the surface of platinum. 


\subsection{Introduction to gold nanoparticles}

Gold nanoparticles (AuNPs) have significantly optical and electronic properties that differ from bulk metal or molecular compounds due to quantum size effects. As the size of the AuNP decreases to the same order of magnitude as the de Broglie wavelength of valence electrons, the electrons are confined in a metallic-like box. Because of this confinement, the interaction of electrons with the electromagnetic radiation is very different compared to bulk gold.

Gold nanoparticles are usually synthesized by the Turkevich method ${ }^{140,141}$ in aqueous solution or by the Brust-Schiffin method ${ }^{142}$ in organic solvents by reduction of precursor salt in the presence of a capping agent. In the Turkevich method, citrate was used both to reduce the gold salt and to stabilize the formed nanoparticle (Figure 5). The ratio of citrate to gold salt can be varied to synthesize AuNPs ranging in size from 3 120 $\mathrm{nm}$.

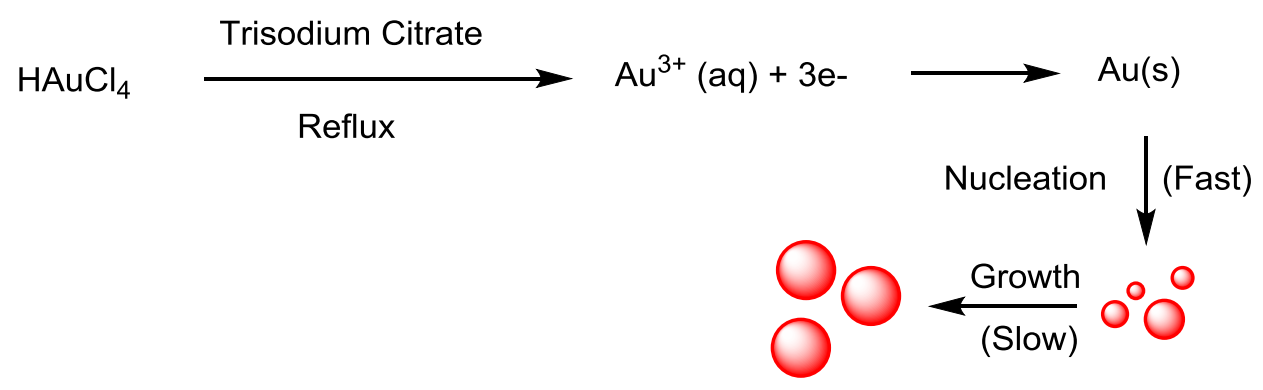

Figure 5. Schematic representation of AuNP preparation through citrate reduction.

\subsection{Optical properties of gold nanoparticles}

Citrate-capped 13-nm AuNPs are most commonly used, as a consequence of their strong absorption of green light at $520 \mathrm{~nm}$. The color of the AuNPs is governed by surface plasmon resonance (SPR) effects. ${ }^{14-145}$ When a metal nanoparticle is exposed to light, the oscillating electromagnetic field of the light induces collective coherent 
oscillation of electrons. The amplitude of oscillation reaches a maximum at a specific SPR frequency. AuNP absorbs and scatters light at the SPR wavelength; this wavelength, and thus the color produced by the AuNP, is affected by nanoparticle size, shape, dispersal in a solvent, and capping agent. When AuNP size increases from 13 to $100 \mathrm{~nm}$, its color changes from red to blue due to red-shifting and broadening of the surface plasmon band. ${ }^{146,147}$ Similarly red-shifting of about $130-180 \mathrm{~nm}$ relative to the spherical particle wavelength is seen with branched gold nanocrystals ${ }^{148}$ or nanostars. ${ }^{149}$ Surface plasmon resonance frequency is also sensitive to the nanoparticle aggregation state; when particles are sufficiently close together, red-shifting of wavelength results in a color change. The well-dispersed AuNPs can be brought together in the presence of targets such as lysine brings the mercury coated AuNPs to render AuNP aggregation (Figure 6).
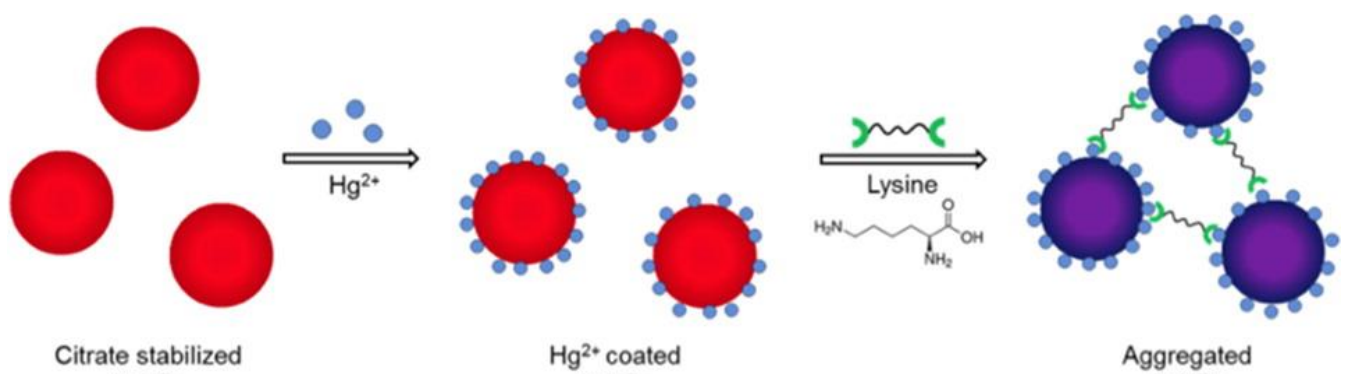

$\mathrm{Hg}^{2+}$ coated AuNPs

$$
\text { AuNPs }
$$
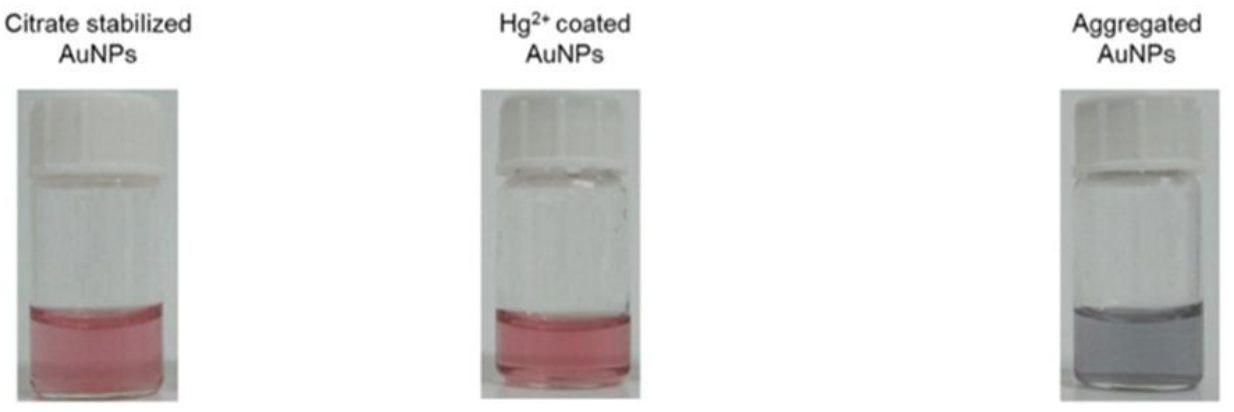

Figure 6. The shifting of color of mercury ion coated AuNPs from red to blue due to aggregation caused by target Lysin. ${ }^{150}$ 


\subsection{AuNP-based colorimetric sensing}

The color of AuNPs is five or six orders of magnitude stronger than most strongly absorbing organic dye molecules, which is enough to visualize by naked eye. The difference in color of individual AuNPs (red) and aggregated AuNPs (purple or blue) has been used as a platform for the colorimetric sensing of target analytes that trigger either the aggregation or re-dispersion of AuNPs via a variety of strategies.

\subsubsection{Functionalization of gold nanoparticles for enzyme activity}

One characteristic feature of AuNPs is the ease with which surface functionalization can be implemented for selective binding and detection of target molecules. Wellestablished methods have been developed for the modification of AuNPs with DNA, ${ }^{151}$ amino acids, ${ }^{152}$ peptides, ${ }^{152}$ antibodies, ${ }^{153}$ ATP. ${ }^{154}$ Such functionalized AuNPs have been used for various applications, including the sensing of enzyme activity and study of enzyme inhibitors for drug development. Such assays are designed so that the substrate and product of an enzymatic reaction have different effects on AuNP stability. Typically, the substrate is immobilized on the surface of the AuNP without affecting its stability, so that it retains its red color. The enzymatic reaction alters the structure of the substrate in a manner that affects AuNP stability, imparting purple or blue color. Substrates implemented to date include DNA ${ }^{155,156}$ for endonuclease activity and methyltransferase assay, peptides for kinase assays, ${ }^{157,158}$ and ATP ${ }^{154}$ for phosphatase activity assays.

Wang et al. ${ }^{157}$ used peptide-modified AuNPs for the colorimetric analysis of two different kinase enzymes and their inhibitors. The peptide substrate was biotinylated to link it with streptavidin-modified AuNPs. In the absence of enzyme or in the presence of inhibitor, the substrate remained intact on the AuNP surface, preserving their red color because of electrostatic repulsion between peptide molecules. In the presence of 
enzyme, the substrate was removed from the AuNPs, inducing aggregation and a color change from red to blue.

\subsection{DNA-functionalized AuNPs for colorimetric enzyme detection}

Deoxyribonucleic acid-functionalized gold nanoparticles (DNA-AuNPs) have been used in gene therapy, drug delivery, and sensing of DNA, ${ }^{159}$ metal ions (Pb), ${ }^{160}$ and small molecules. These DNA-AuNPs have been used in conjunction with different DNAmodifying enzymes either to enhance the target signal or to detect the enzyme itself. In theory, any DNA-modifying enzyme can be used, and assays described to date have employed exonuclease III, DNase $1,{ }^{161}$ and various endonucleases. ${ }^{155}$ Several different mechanisms have been used to monitor colorimetric enzymatic assays based on DNAAuNP assembly substrates.

\subsubsection{Cross-linking mechanisms}

Deoxyribonucleic acid substrate can be conjugated to the surface of AuNPs to enable inter-particle DNA crosslinking. The crosslinked DNA gives rise to DNA-AuNP aggregates which serve as the substrate for Dnase I endonuclease; enzymatic cleavage converts the aggregates into dispersed AuNPs, resulting in a purple-to-red color change, as shown in figure 7 . The color change offers a readout for the enzymatic assay.

Xu et al. ${ }^{155}$ conjugated two different DNA sequences onto the surface of the AuNPs using thiol-gold chemistry. One sequence was attached to one set of AuNPs by its 5' end, whereas the other was attached to another set of AuNPs by modifying its 3' end with a thiol group. These two sets of modified DNA-AuNPs were mixed and heated at $90^{\circ} \mathrm{C}$ for 10 minutes and slowly cooled to room temperature to form a crosslinked aggregate containing 30-nt duplexes that serve as a substrate for the DNase enzyme. Incubation with DNase led to dissociation of the crosslinking, enabling AuNP dispersal and a purple-to-red color change. To simplify this assay and to make it general for all 
endonucleases, Guangtao et al. ${ }^{156}$ used free DNA as a linker to crosslink a single type of ssDNA-modified AuNPs. The use of crosslink greatly simplified the assay, and the recognition sequence of the linker DNA can be changed to develop assays for other endonucleases.

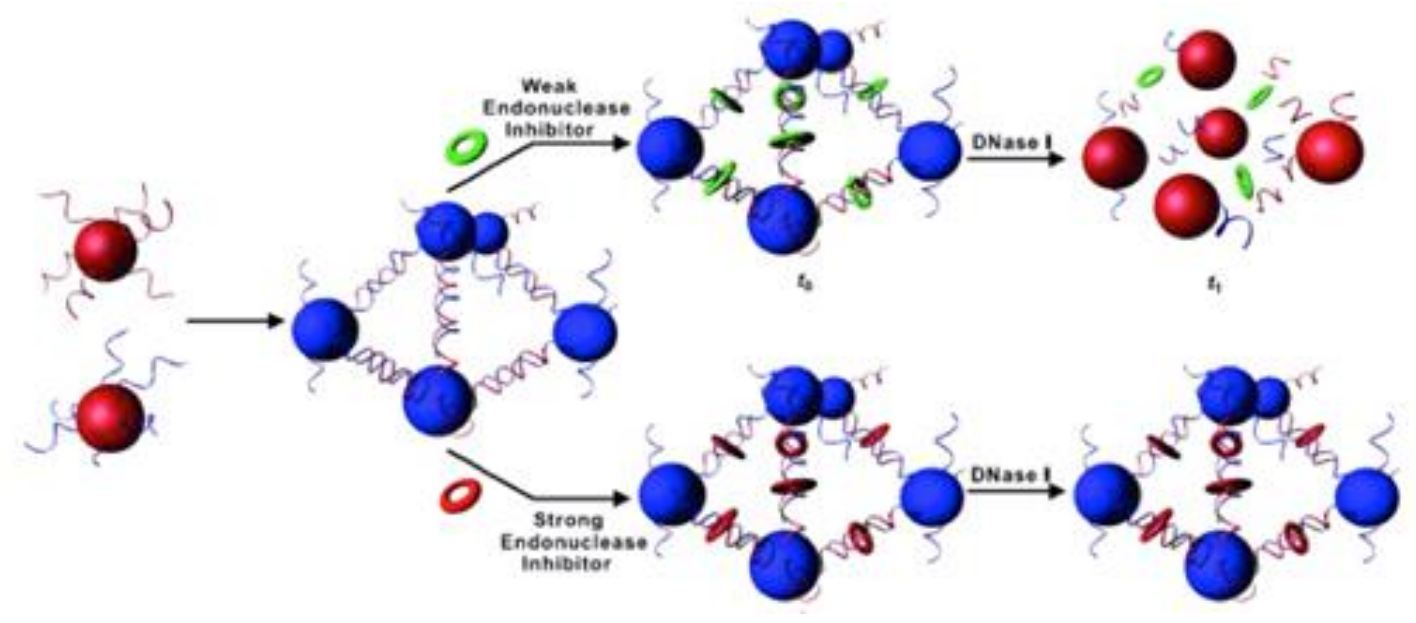

Figure 7. Cross-linked DNA-AuNPs as a colorimetric assay for Dnase I endonuclease enzyme. ${ }^{155}$

\subsubsection{Non-crosslinking-based mechanisms}

To further simplify the assay, one can also employ a non-cross-linking mechanism. Zhao et al. ${ }^{162}$ initially reported a colorimetric assay that used non-crosslinking-based aggregation of AuNPs to monitor phosphatase-mediated removal of phosphate groups. The same group extended the approach to analyze the endonuclease DNase I based on removal of enzyme-specific DNA substrates from the surface of the AuNPs by the enzyme. ${ }^{161}$ The assay was based on hybridization of a single type of DNA-AuNP with a complementary DNA (cDNA) strand. The DNA substrate is prepared directly on the AuNP surface by hybridizing the DNA-AuNP with the cDNA in solution. The AuNPs are well dispersed, imparting the characteristic red color due to electrostatic repulsion 
between the DNA molecules on its surface. In the presence of DNase I enzyme, the DNA substrate is degraded, leading to aggregation as a result of removal of electrostatic charge from the AuNP surface. The change in color from red to blue is used to measure the enzyme activity.

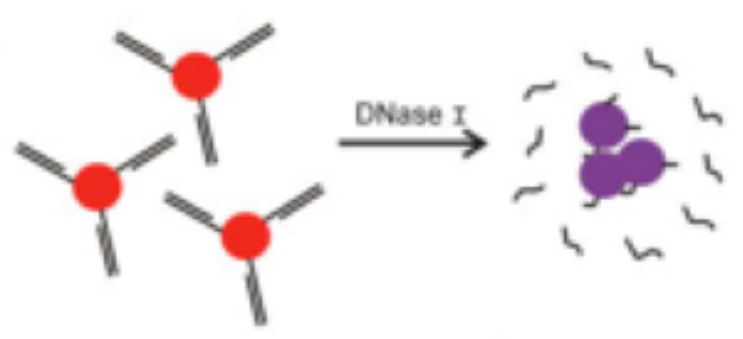

Figure 8. Non-crosslinking-based mechanism for measuring DNase I enzyme activity with DNA-modified AuNPs in a colorimetric assay. ${ }^{161}$

One problem associated with the use of DNA-AuNPs for colorimetric enzyme assays is the limited accessibility of conjugated DNA to enzymes compared to solution-based assays. However, DNA is more accessible to enzyme in non-crosslinking-based assays compared to crosslinking-based assays because of the greater degree of particle dispersal.

\subsection{DNA-AuNP as a substrate for exonuclease III}

Exonuclease III is a double-stranded (ds) DNA-specific enzyme. dsDNA-AuNP substrates for exonuclease III can be prepared by hybridization of ssDNA probemodified AuNPs with a complementary target. ${ }^{163-165}$ Usually, the 5' end of the DNA is modified with a thiol group and attached to citrate-coated AuNPs using standard goldthiol chemistry. This probe DNA-AuNP substrate is then hybridized with its cDNA. In addition to reaction conditions such as temperature, concentration of magnesium, and ionic strength of buffer, the performance of this enzymatic colorimetric assay depends 
mainly on the surface density of DNA probes on the AuNPs and their cDNA hybridization efficiency.

\subsection{Thiols as a back-filler}

DNA surface density affects the AuNP colloidal stability, ${ }^{166}$ DNA orientation, and steric hindrance ${ }^{167,168}$ and determines hybridization efficiency, ${ }^{169,170}$ and hence reaction time, in enzyme colorimetric sensors. Therefore, different thiols have been used to optimize surface coverage for faster AuNP aggregation.

The use of thiols as a backfiller is very common on gold surfaces. ${ }^{171,172}$ DNAmodified gold surfaces are immersed in mercaptohexanol $(\mathrm{MCH})$ to remove nonspecifically adsorbed DNA and to block the uncovered gold surface. Such treatment results in higher hybridization efficiency and improved sensitivity compared to untreated gold surfaces. For example, Luo et al. ${ }^{172}$ used $\mathrm{MCH}$ as a back filler after modifying gold electrodes with thiolated DNA to align the DNA monolayer and increase hybridization efficiency. Such MCH treatment has also been used on DNA-AuNP surfaces. Park et al. ${ }^{168}$ showed that $\mathrm{MCH}$ treatment of DNA-AuNPs gives rise to a hybridization-friendly stretched oligonucleotide conformation. Zhao et al. ${ }^{161}$ also showed that $\mathrm{MCH}$ treatment could selectively remove DNA molecules from the DNA-AuNP substrate to increase the sensitivity of the colorimetric reaction. Wu et al. have shown that medium surface density is required for optimum cDNA hybridization at the surface of gold electrodes, as hybridization efficiency decreases at higher probe density due to steric hindrance. ${ }^{173}$ Similarly, Oh et al. ${ }^{174}$ showed that moderate surface coverage significantly enhances the hybridization rates of DNA-AuNP relative to higher surface coverage.

\subsection{Significance of exonuclease III}

Exonuclease III is prokaryotic DNA-modifying enzyme. ${ }^{175}$ It is a major apurinic/apyrimidinic (AP) endonuclease and cleaves phosphodiester bonds in duplex 
DNA immediately 5' to abasic sites to produce a $3^{\prime}-\mathrm{OH}$ group. ${ }^{176-178}$ It has structural and functional similarity with other class II AP endonucleases such as human APE1 and yeast Apn1, and is often used as a model enzyme for mechanistic studies. ${ }^{179,180}$ Exo III is also a 3'-to-5' exonuclease and removes mononucleotides from the 3' end of nicks in dsDNA. ${ }^{178,181,182}$ It also has RNase $\mathrm{H}$ and 3' phosphatase activity.

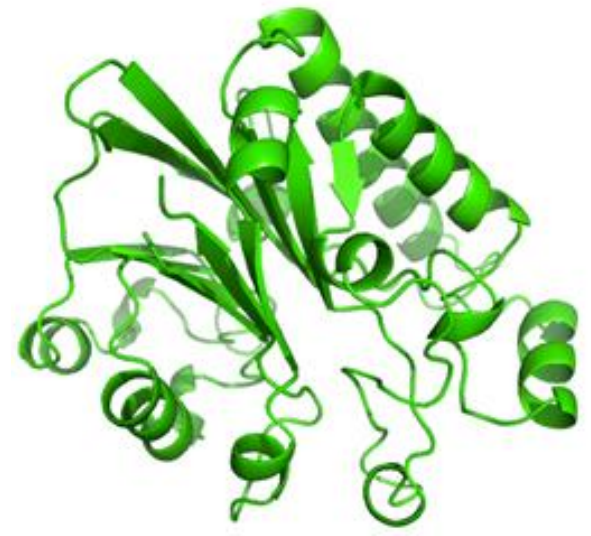

Figure 9. X-ray crystal structure of exonuclease III from E. coli. ${ }^{178}$

Figure 9 represents the X-ray crystal structure of E. coli exonuclease III. Exo III is a metal-dependent enzyme, and either $\mathrm{Mg}^{2+}$ or $\mathrm{Mn}^{2+}$ or $\mathrm{Ca}^{2+}$ is essential for its activity. ${ }^{183}$ Metal-bound Exo III achieves phosphate bond cleavage via nucleophilic attack. ${ }^{178}$ Accordingly, its 3'-to-5' exonuclease activity hydrolyses phosphodiester bonds in DNA from the 3' OH group, as shown in Figure 10. 


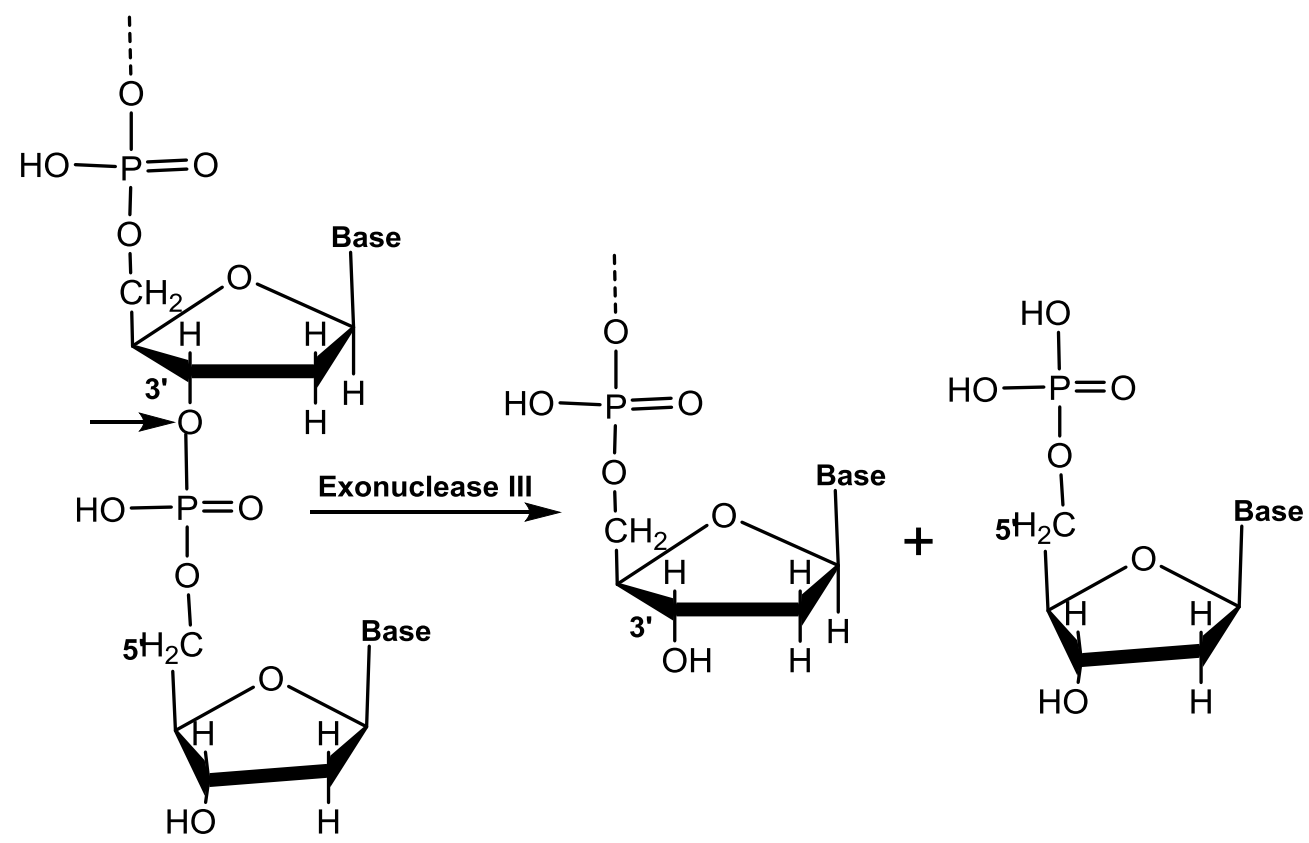

3' hydroxy terminus

Figure 10. Hydrolysis of phosphodiester bonds at the 3' OH group by Exo III.

Exo III's AP endonuclease activity hydrolyses phosphodiester bond immediately 5 ' to the abasic site to produce 3' $\mathrm{OH}$ and 5' -deoxyribose moieties, and is thus classified as a class II AP endonuclease. Enzymes from this class have been studied as attractive targets for drug development in some cancers because of their important role in proliferation, and they are viewed as a diagnostic marker for early cancer detection. Altered expression and localization of these enzymes have been established as common features in different neoplastic diseases, suggesting that these enzymes may have prognostic and/or predictive value in cancer. ${ }^{184-186}$ Similarly, overexpression of $3^{\prime}-5^{\prime}$ exonucleases in human cells is possibly related to increased longevity, owing to stabilization of cellular mutation rates, whereas organisms that lack 3'-5' exonucleases are more susceptible to cancer, especially under stress conditions. ${ }^{187,188}$ Exo III is also often used in enzyme-assisted target recycling (EATR) for the detection of DNA, protein, 
and small molecule targets. ${ }^{189-191}$ Although in principle APE1 can also be used in such assays, Exo III has been preferentially used for the development of colorimetric assays due to its commercial availability, higher catalytic efficiency and higher 3'-to-5' exonuclease activity relative to APE1.

\subsection{Current approaches to Exo III detection}

Several methods have been developed to detect Exo III activity. The most common traditional method is gel electrophoresis-based radiographic ${ }^{192,193}$ or fluorescence assays, in which labeled DNA is treated with exonuclease III and the digested products are then separated via gel electrophoresis. However, these assays require stringent safety procedures to control exposure to radiation or biohazard dyes. Furthermore, these assays are laborious, and requires lengthy time for gel preparation and analysis.

As an alternative, solution-based fluorescence assays have been developed for quick enzyme analysis. Wu et al. ${ }^{194}$ used fluorescently-labeled molecular beacons as a DNA substrate for the detection of exonuclease activity. The hairpin structure of the molecular beacon was modified with a fluorophore (FAM) at its 5' end and a quencher (BHQ1) at its 3' end. In the absence of enzyme, the fluorescence was quenched in the absence of enzyme due to Forster resonance energy transfer (FRET). However, in the presence of enzyme, it releases the quencher into due to Exo III's 3' to 5' activity, increasing its fluorescence in solution. This change in fluorescence was used as a measure of activity of exonuclease III. Alternatively, Su et al. ${ }^{195}$ used stacked guanines as a quencher instead of BHQ1 to reduce the cost of the assay. Label-free fluorescence assays have also been developed, based on formation of G-quadruplex structures upon Exo III reaction with linear DNA sequences. The enzyme activity was then monitored by measuring the fluorescence of G-quadruplex-specific crystal violet dye ${ }^{196}$ or $\mathrm{Tb}_{2} \mathrm{O}_{3}{ }^{197}$ 
The use of DNA-AuNP substrates for Exo III detection greatly simplifies the assay, as the activity of the enzyme can be monitored by the naked eye without the need for an instrument. The high extinction coefficient results in an assay with sensitivity comparable to the fluorescence assay. Exonuclease III has been used for target recycling in AuNPbased colorimetric assays, ${ }^{165}$ but no colorimetric assays have been developed to date for the detection of its activity. 


\section{Chapter 3. Paper-Based, Carbon Nanotube (CNT) Thin Film for Electrochemical Detection of Guanine and its Nucleotides}

\subsection{Introduction}

Carbon nanotubes (CNTs) have been widely used as an electrode material to promote electron transfer rate between electro-active molecules and underlying electrode materials. ${ }^{198-201}$ The single-walled carbon nanotube (SWCNT) has well-defined electronic properties with high surface area $\left(1600 \mathrm{~m}^{2} \mathrm{~g}^{-1}\right)$ and high current density $\left(109 \mathrm{Acm}^{-2}\right)$ that can form ensemble with high conductance. ${ }^{1-3,5,8}$ Therefore, it improves the electron transfer rate by increasing the electrode surface area and reduces electrode fouling, ${ }^{202}$ leading to sensitive and selective detection of target molecules.

The control of orientation and density are two key parameters for the performance of SWCNT electrodes. Vertically aligned nanotubes formed either by chemical vapor deposition, ${ }^{21-23}$ or by self-assembly, ${ }^{14}$ have better performance than randomly oriented SWCNTs as a result of the larger exposure of edge plane like structures (end) as compared to basal plane like structure (sidewall). ${ }^{9-11}$ However, it requires specialized equipment, restricted to certain substrates such as silicon, quartz, metals and carbon fibers. The process is complicated, expensive and is not suitable for mass production. As an alternative, randomly oriented SWCNT was either packed as a paste ${ }^{29-33}$ or deposited on electrode surface either by casting, ${ }^{39,40}$ or dip coating. ${ }^{34-37}$ As compared to vertically aligned tubes, it is simple, cost-effective, suitable for mass production and compatible to a wide variety of substrates. Usually, the active electrochemical sites exist on side walls, or its edge is utilized to achieve the catalytic response. ${ }^{203}$

Because of excellent electrochemical properties of SWCNT, it has been used as an electrode material to increase electron transfer rate between the electroactive molecule and electrode material on the surface of metals, ${ }^{25,38}$ glassy carbon electrode, ${ }^{39}$ quartz, ${ }^{204}$ 
and graphite. ${ }^{205}$ Although these electrode materials are widely used, there is growing interest on deposition of CNT on the paper substrate. The use of paper instead of traditional electrode materials has following advantages: 1) It eliminates the requirement of any pre- or post-treatment of electrodes; 2) It significantly reduces the cost of an electrode from $\sim \$ 100$ to $<\$ 1 ; 3)$ It is disposable and environment-friendly. Fabrication of such paper-based electrode material is key for the development of paper-based electrochemical sensors which have proven as a simple, low-cost, portable and disposable alternatives of traditional analytical methods of target detection. ${ }^{206}$

The Vacuum filtration method ${ }^{207}$ is one of the simplest and well-established method of making a CNT thin film on a porous paper substrate. The density can be controlled just by controlling the volume of the CNT used. It has been illustrated in various reports that this process can form very thin film $(\sim 50 \mathrm{~nm})$ with high homogeneity and integrity on paper substrates. ${ }^{55,207,208}$ Because of its simple fabrication, stability, and porous structure, it has been used as a gas sensor and as a filter. Although paper in general, is not considered to be a good substrate for an electrode material, there are few reports about the use of vacuum filtered SWCNT paper as electrode material. For example, Wang et al. ${ }^{209}$ investigated the capacitance of SWCNT paper in supporting electrolytes. Li et al. ${ }^{210}$ studied the electron transfer properties of SWCNT paper in ferricyanide redox probe. They showed that performance of the electrode depends on the surface density and oxides on the nanotube surface contribute to the capacitance current. $\mathrm{Hu}$ et $\mathrm{al}^{55}$ showed wide potential window of SWCNT paper and its successful use as a dopamine sensor.

It has been reported that electrochemical response of the modified electrode depends on the surface density of CNT as well as porosity of electrode material. Porosity has been attributed to the better performance of graphite pencil electrode as 
compared to commonly used glassy carbon electrode for the detection of guanine signal when both the electrode materials are modified with multi-walled CNT (MWCNT). ${ }^{211}$ The SWCNT thin film network by vacuum filtration method represents a system that can be easily prepared at very low fractional surface coverage on paper substrate with high porosity.

Herein, we studied the feasibility of vacuum filtered Pure Single-Walled Carbon Nanotube (P-SWCNT) thin film as an electrode on the paper substrate and used this paper-based P-SWCNT film for electrochemical sensing. An atomic force microscope (AFM) and sheet resistance were used to characterize the surface of SWCNT paper. We studied the change in electrochemical properties of P-SWCNT paper as a function of its density for sensor application. By lowering the surface density, we were able to reduce the background current, which is significant for electroanalytical applications. The low density and highly porous PSWCNT paper were used for sensitive detection of guanine and its nucleotides.

\subsection{Materials and Methods}

Chemicals and Apparatus. Guanine $(G)$, sodium salt of guanosine 5'monophosphate(GMP) and sodium salt of Guanosine 5'-triphosphate(GTP) were obtained from MP biochemical, Ohio USA. Puretubes SWCNTs were purchased from Nanolntegris (Menlo Park, CA, USA). Potassium chloride, sodium dodecyl sulfate (SDS), sodium hydrogen phosphate and dihydrogen sodium phosphate were purchased from Sigma-Aldrich. Mixed cellulose (MCE) membrane $(47 \mathrm{~mm}$ diameter, $100 \mathrm{~nm}$ pore size and $100 \mu \mathrm{m}$ thickness) was purchased from Millipore Corporation (Billerica, MA, USA). All other chemical reagents were purchased from Sigma-Aldrich and used without further purification. All aqueous solutions were prepared using deionized water from a Milli-Q water purification system (Millipore Corporation, Billerica, MA, USA). Guanine stock 
solution was prepared in $0.1 \mathrm{M} \mathrm{NaOH}$ whereas GMP and GTP stock solutions were prepared in $\mathrm{DI}$ water. All the solutions were stored in a refrigerator at $4^{\circ} \mathrm{C}$ before use.

Fabrication of thin P-SWCNT film. The P-SWCNT thin film was prepared by reported vacuum filtration method ${ }^{207}$ with slight modification. Briefly, concentrated pure Single-walled carbon nanotube (P-SWCNT) from Nano Integris (Arc discharge method, $1.4 \mathrm{~nm}$ diameter, 1-micron length) was diluted in 1\% SDS solution and dispersed using sonication $(335 \mathrm{~W}, 50 / 60 \mathrm{~Hz}$ ) for 10 minutes. The well-dispersed P-SWCNT solution was then poured onto MCE membrane placed in vacuum filtration apparatus. For optimum uniformity, the solution was left to sit for 5 minutes before applying vacuum. The vacuum was then applied slowly for 60 minutes to collect P-SWCNT network on the membrane surface. The surfactant was washed away using an excess of DI water. The film was finally dried in vacuum filtration apparatus overnight.

Surface characterization. The surface morphology of MCE membrane and PSWCNT thin film loaded on MCE membrane was characterized by using atomic force microscope (AFM) (Veeco Metrology, NY, USA) in tapping mode. To measure the thickness; the thin film was transferred into a glass substrate by reported acetone evaporation method. ${ }^{207}$ Average film thickness was obtained from AFM by measuring the height difference of P-SWCNT thin film and glass substrate at the edge of the thin film.

Transfer of CNT thin film on glass substrate. The P-SWCNT thin film was transferred from paper substrate to glass substrate by reported acetone evaporation method $^{207}$ to measure the thickness of the fabricated CNT films. The glass slide $(0.8 \mathrm{~cm}$ $X 1.6 \mathrm{~cm}$ ) was cleaned with acetone, water and ethanol using sonication each for 5 minutes. P-SWCNT films $(0.8 \mathrm{~cm} \times 0.8 \mathrm{~cm})$ was rinsed with DI water and placed on the glass substrate facing it down. The glass slide with samples was then placed in between two bigger glass slides and pressed with heavy weight for overnight to stick the P- 
SWCNT film onto the glass substrate. The glass substrate attached with the P-SWCNT film was carefully placed into a home-made acetone evaporation apparatus in a fume hood. The home-made device contains a $500 \mathrm{ml}$ beaker with $\sim 250 \mathrm{ml}$ of acetone in it along with metal mesh on top of acetone solution to hold the sample. The beaker was covered with a watch glass to prevent acetone evaporation during heating. The sample was heated at $85{ }^{\circ} \mathrm{C}$ for one hr. After one hour of gentle heating, the glass substrate containing P-SWCNT thin film was placed vertically and was heated further at $115^{\circ} \mathrm{C}$ for 4 hrs to completely dissolve the filter paper from P-SWCNT film. After that, the glass substrate containing P-SWCNT film was carefully taken out and subsequently dipped in fresh acetone and methanol each for 15 mins. The film was then air dried for $1 \mathrm{hr}$ before the measurement.

Sheet resistance measurement. Sheet resistance was measured using Keithley 4200 Semiconductor Characterization system using four-point probe set up. A thin layer of aluminum foil was used to connect the edge of the P-SWCNT film to complete the circuit with source meter.

Fabrication of electrode from the thin P-SWCNT film. One thin P-SWCNT film was used to make $\sim 24$ different working electrodes by cutting the film into small pieces $(0.3 \mathrm{~cm} \times 0.9 \mathrm{~cm})$. Electric contact was made at one end of the piece by using copper wire. Silver paint (TED PELLA) was used as a connector between copper wire and paper-based P-SWCNT film. The silver paint and copper wire were sealed using Parafilm. The Para-film covering was adjusted to define the geometrical surface area of working electrode as $0.3 \mathrm{~cm} \times 0.6 \mathrm{~cm}$.

Electrochemical experiments. All electrochemical experiments were performed using $\mathrm{CHI}$ electrochemical station ( $\mathrm{CH}$ Instruments, Inc., Austin, USA). The paper-based P-SWCNT thin film electrode, $\mathrm{Ag} / \mathrm{AgCl}$ electrode, and Pt wire were used as a working, 
reference and counter electrode, respectively. All solutions were prepared in $0.1 \mathrm{M}$ phosphate buffer at $\mathrm{pH} 7.0$ and used without purging with inert gas and without a Faraday cage at room temperature. The parameters for differential pulse voltammetry were as follows: pulse width $=0.125 \mathrm{~S}$, pulse period $=0.25 \mathrm{~S}$ and pulse amplitude $=$ $0.05 \mathrm{~V}$.

\subsection{Results and Discussion}

\subsubsection{Fabrication of paper-based P-SWCNT thin film}

The paper-based P-SWCNT thin film was prepared by depositing P-SWCNT on porous mixed cellulose ester membrane using vacuum filtration (VF) method (Figure 11A). The VF method was chosen because of its ability to form very thin and uniform carbon nanotube network on the porous paper substrate. The natural adjustment of local permeation and deposition rate of SWCNT solution due to vacuum force during filtration ensures the uniformity of the thin film (Figure 11B). The film thickness could be controlled just by varying the volume and/or amount of P-SWCNT solution during filtration procedure. Furthermore, the dispersing agents such as surfactants could easily be removed by washing with water.

\subsubsection{Characterization of paper-based P-SWCNT thin films}

Previous studies have shown that density of CNT can be used to manipulate electrochemical properties for various applications. ${ }^{55,209,210}$ Specifically, the studies have shown that lower density of CNT minimizes the capacitance current, which is very significant for detection of electroactive targets. To find the effective surface density, we prepared P-SWCNT thin films with the surface density ranged from 2.2 to $17.6 \mu \mathrm{g} / \mathrm{cm}^{2}$. The amount of P-SWCNT used was calculated with the assumption that no P-SWCNT was lost during the film preparation or washing. Note that our P-SWCNT density on the paper was at least $\sim 400 \mathrm{X}$ less than that prepared by Joseph, ${ }^{209} \sim 10 \mathrm{X}$ less than that 
used by $\mathrm{Li}^{210}$ and $\sim 6$ times less than that used by Chengguo $\mathrm{Hu}^{55}$

A

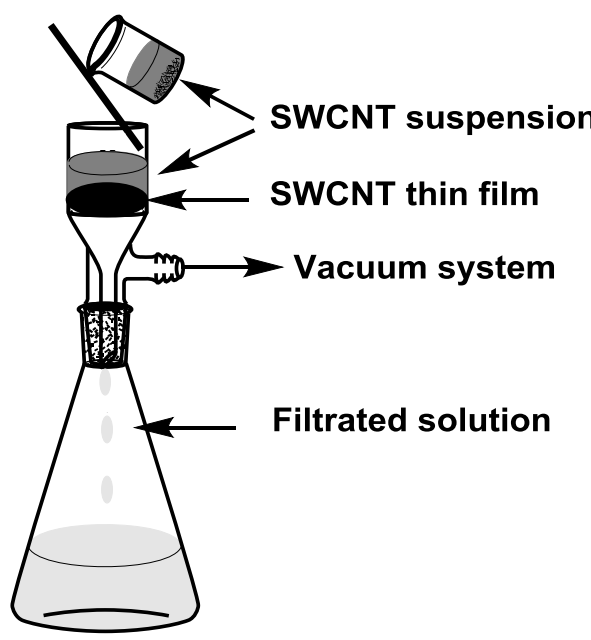

B

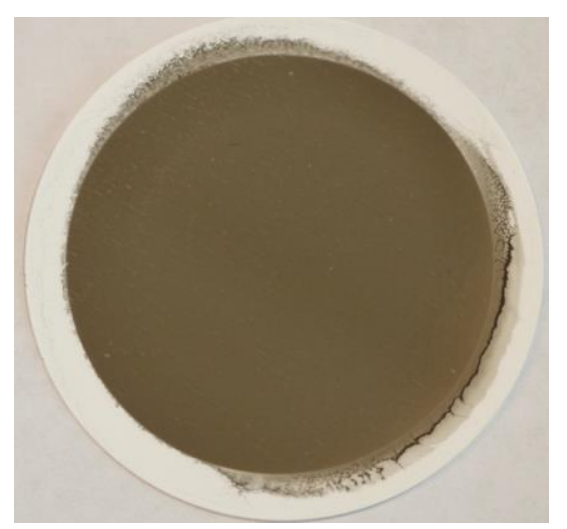

Figure 11. Fabrication of paper-based P-SWCNT thin film. (A) Schematic representation of vacuum filtration (VF) method. (B) photograph of paper-based P-SWCNT thin film.

\subsubsection{The morphology of paper-based P-SWCNT thin film}

We studied the morphology of our paper-based P-SWCNT using atomic force microscope (AFM). Figure 12 shows the tapping mode AFM images of the paper-based P-SWCNT thin films with different surface densities starting from 2.2 to $17.6 \mu \mathrm{g} / \mathrm{cm}^{2}$. Irrespective of densities, all images showed individual SWCNTs were randomly oriented and interconnected to form porous SWCNT network (Figure 12). The highly porous structure is in contrast to the compact structure of casted CNT films on glassy carbon electrode (GCE). ${ }^{41}$ The analysis of AFM pictures also showed that porous structure was more prominent in lower density films as compared to higher density films. The higher degree of asymmetry of surface height distribution of different films indicated that higher electrochemical surface area could be achieved with low-density SWCNT films. 


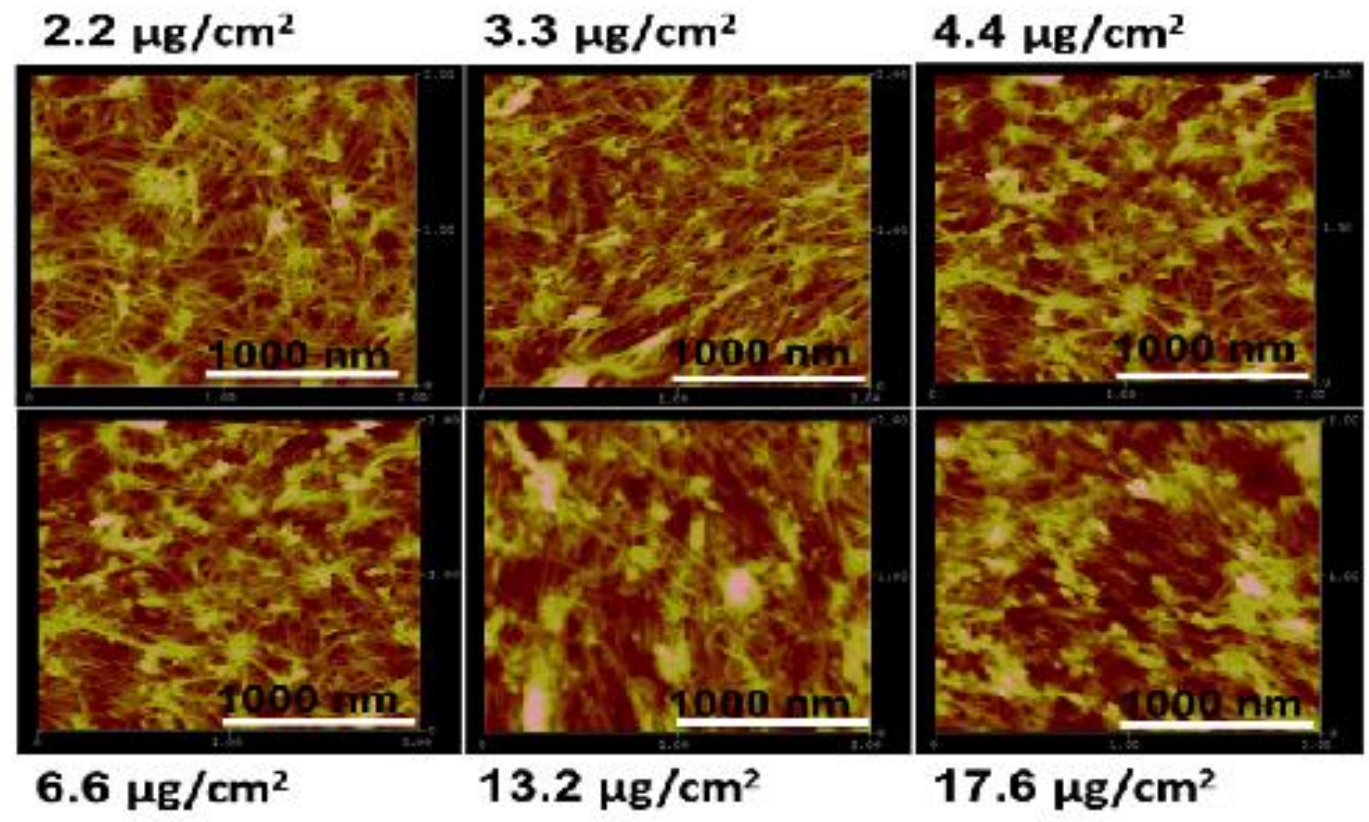

Figure 12. Surface morphology of paper-based P-SWCNT thin films. Atomic force microscopy images of P-SWCNT thin films with different surface densities varying from $2.2 \mu \mathrm{g} / \mathrm{cm}^{2}$ to $17.6 \mu \mathrm{g} / \mathrm{cm}^{2}$.

\subsubsection{The thickness of paper-based P-SWCNT thin film}

The thickness of the paper-based P-SWCNT thin film was further characterized. For this, the P-SWCNT thin film was transferred into a glass substrate by acetone evaporation method. The height profile difference of P-SWCNT film and glass substrate, during AFM imaging, was used to derive the thickness of the film. Figure $13 \mathrm{~A}$ indicates the representative height profile used to calculate the thickness of the P-SWCNT film. The height profile of each P-SWCNT film showed that all films were relatively uniform. The thickness of the films prepared with surface density in the range of 2.2 to17.6 $\mu \mathrm{g} / \mathrm{cm}^{2}$ lies in the range of $\sim 46$ to $230 \mathrm{~nm}$. Additionally, the linear relationship between thickness and surface density indicates the proper control of SWCNT density during the fabrication process. 
A

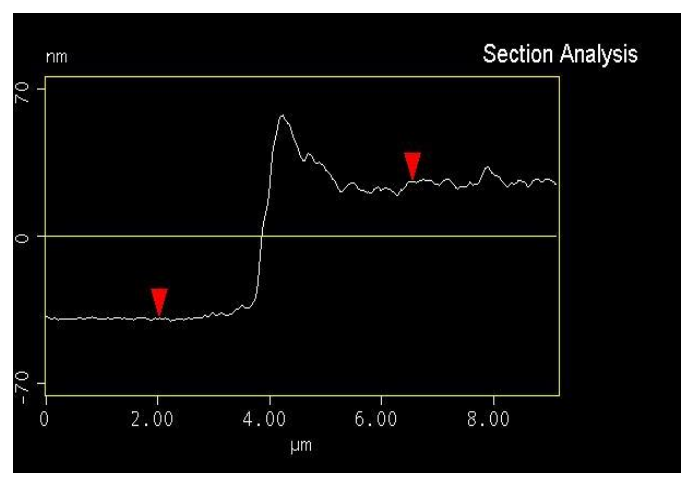

B

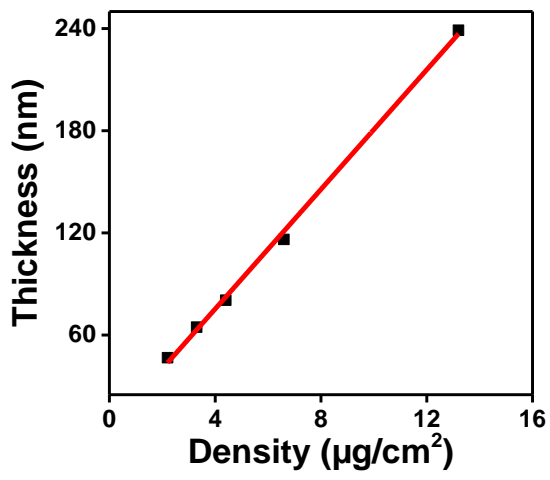

Figure 13. Characterization of thickness of paper based P-SWCNT thin film. (A) Height profile of P-SWCNT from AFM image. (B) Thickness of various P-SWCNT films calculated from height profile as a function of surface density.

\subsubsection{The sheet resistance of paper-based P-SWCNT thin film}

The use of paper-based low-density-SWCNT thin film as an electrode is limited by its film conductivity. Usually, the lower value of sheet resistance suggests a higher number of conductive pathways for electron transfer within the P-SWCNT film. To investigate the relationship between film resistance and surface density of the CNT film and to find out optimum P-SWCNT density required to achieve excellent conductance, we measured the sheet resistance of various paper-based P-SWCNT films. Figure14 represents the plot of sheet resistance as a function of the surface density of P-SWCNT in the range of 2.2 to $13.2 \mu \mathrm{g} / \mathrm{cm}^{2}$. It showed that sheet resistance decreases with the increase of surface density of SWCNT from 2.2 to $6.6 \mu \mathrm{g} / \mathrm{cm}^{2}$. The sheet resistance remained constant with further increase of SWCNT density above $6.6 \mu \mathrm{g} / \mathrm{cm}^{2}$. Therefore, $6.6 \mu \mathrm{g} / \mathrm{cm}^{2}$ was used as the minimum SWCNT density required to achieve excellent film conductance.

\subsubsection{Fabrication of paper-based P-SWCNT film electrodes}

The greatest challenge of solution-based methods for the preparation of CNT electrodes is the protection of its inherent electrochemical properties during electrode 


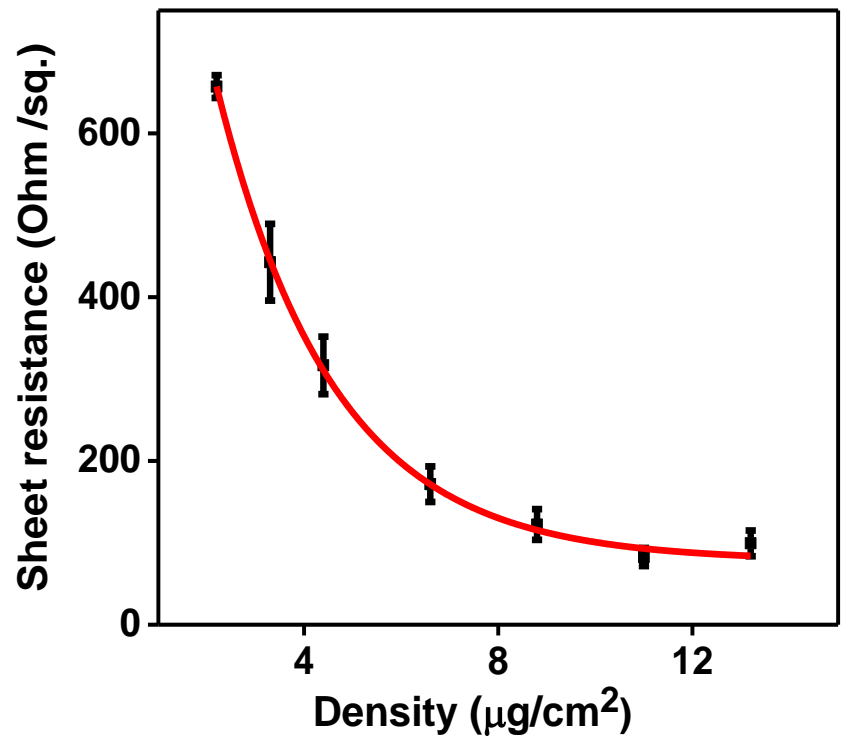

Figure 14. Characterization of sheet resistance of paper-based P-SWCNT thin film. Variation in sheet resistance as a function of surface density of P-SWCNT.

fabrication. In traditional manufacturing methods, CNT was oxidized before dissolving into less soluble aqueous or organic solvents. The use of more soluble surfactant solution was limited because of the difficulty in removing it during fabrication. In contrast, the surfactant molecules used for the CNT dispersion can be washed away easily in our VF method. Furthermore, uniform, porous, well-dispersed and overlapped structure of the P-SWCNT network is ideal for making the CNT-based electrode. To make our PSWCNT thin film electrodes, we cut the P-SWCNT thin film into small pieces and the paper-based thin film pieces were electrically connected with copper wire using silver paint. The geometrical surface area was defined by covering it with parafilm as shown in Figure15.

The fabrication of paper-based P-SWCNT film electrodes brings several advantages as compared to traditional casting methods. It involves just vacuum filtration of aqueous solution on a paper substrate and does not involve the tedious multistep polishing and 
cleaning of electrode material before deposition of the CNT layer. It also brings the possibility of using one film to make mass production of paper-based P-SWCNT (24 electrodes). Furthermore, the use of paper instead of traditional electrode materials significantly reduce the cost, as MCE filter paper is much cheaper than the most commonly used GCE.

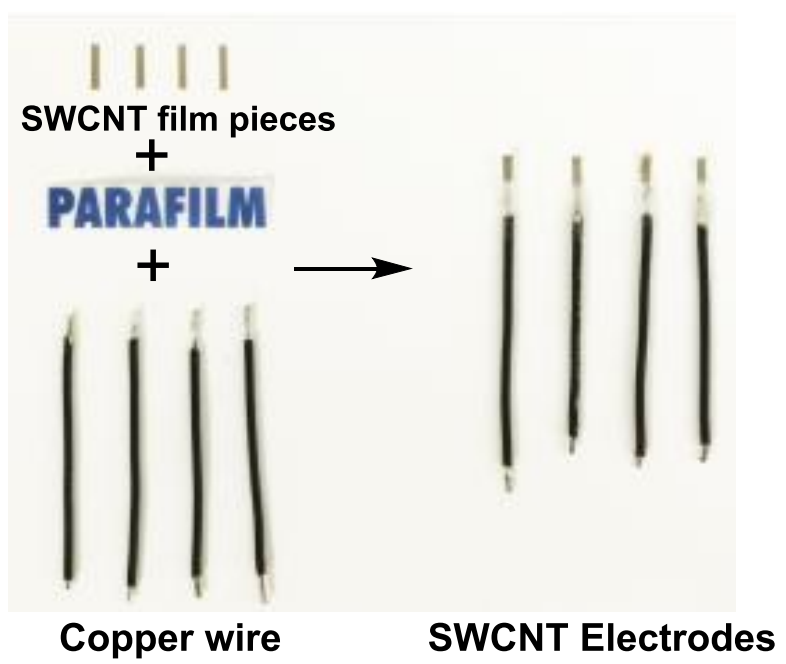

Figure 15. Fabrication of paper-based P-SWCNT electrode. Schematic representation of production of P-SWCNT film electrode from paper-based SWCNT thin film.

\subsubsection{Electrochemical properties of paper-based P-SWCNT film electrode}

To examine the effect of SWCNT density on heterogeneous charge transfer mechanism of the paper-based P-SWCNT film electrode, we calculated the electron transfer rate of our film electrode using cyclic voltammetry. Ferricyanide redox system, which is known to follow inner sphere mechanism, was chosen to account the electron transfer rate of the paper-based P-SWCNT film electrodes. Figure 16A is the representative cyclic voltammogram of the SWCNT thin film electrode obtained in $5 \mathrm{mM}$ $\mathrm{Fe}^{3+} / \mathrm{Fe}^{2+}$ containing $0.1 \mathrm{M} \mathrm{KCl}$ showing both cathodic and anodic peaks. The peak-topeak separation was used to calculate electron transfer rate by Nicholson method. ${ }^{212}$ 

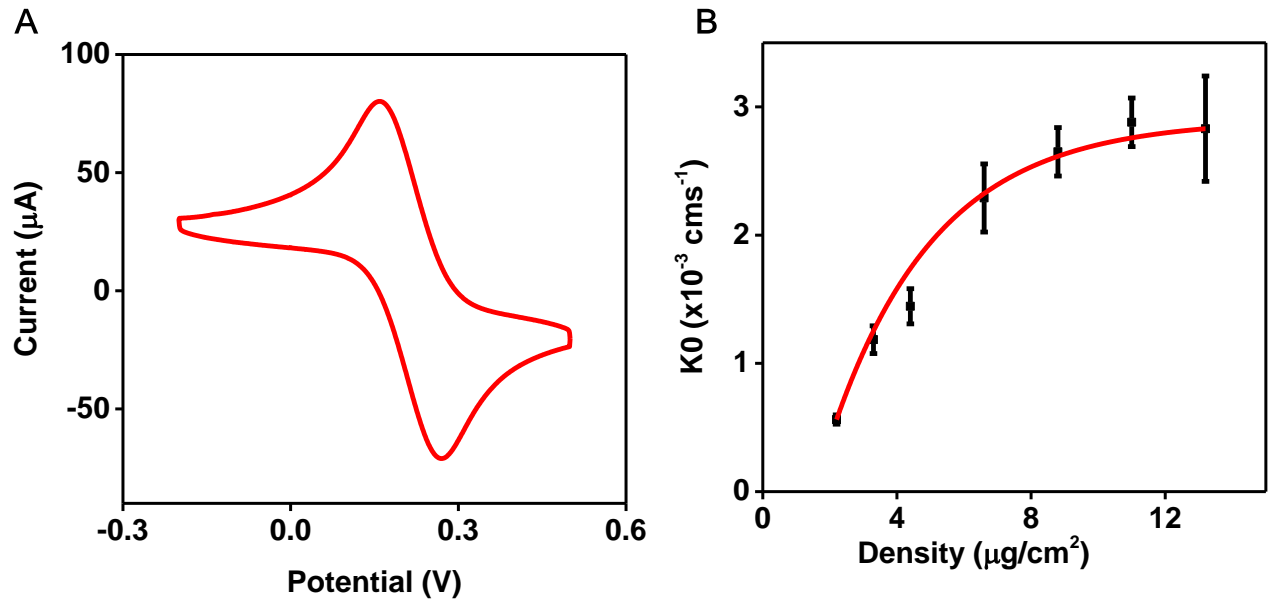

Figure 16. Electrochemical properties of paper-based P-SWCNT electrode. (A) Representative cyclic voltammetry of the paper-based P-SWCNT film electrode in potassium ferricyanide solution and (B) variation of electron transfer rate as a function of surface density of SWCNT.

Figure $16 \mathrm{~B}$ represents the variation in electron transfer rate as a function of the surface density of P-SWCNT. It showed that the maximum electron transfer rate of $2.8 \times 10^{-3} \mathrm{cms}^{-1}$ could be achieved from paper-based P-SWCNTfilm electrode. The electron transfer rate increases with the surface density of P-SWCNT. However, it gets saturated when $6.6 \mu \mathrm{g} / \mathrm{cm}^{2}$ of SWCNT or above was used to prepare the film. Therefore, $6.6 \mu \mathrm{g} / \mathrm{cm}^{2}$ is taken as optimum P-SWCNT density which is consistent with sheet resistance data.

\subsubsection{Electrocatalytic response of paper-based SWCNT thin film electrode}

The electrocatalytic properties of paper-based SWCNT thin film showed that it could be used as an electrode material for detection of an electroactive target. The porous feature of the paper-based P-SWCNT film may allow fast adsorption and/or diffusion of target molecules to/ from the surface of the electrode. These properties of the paperbased P-SWCNT film could be used for the detection of guanine and its nucleotides. 
Guanine and its nucleotides were chosen as target molecules because of their importance in the mechanistic study and diagnosis. The mechanistic study of the guanine can be useful in understanding the oxidative DNA damage and coding of genetic information. Due to their role in various metabolic processes such as enzymatic reactions, an abnormal change in their concentrations is an indicative of mutation of immunity system ${ }^{58,59}$ and often related to different diseases such as cancer ${ }^{60}$ and HIV infection ${ }^{61}$. These molecules are electrochemically active and can be oxidized on the electrode surfaces. It has been reported that those electro-active molecules can be adsorbed on the surface of CNT through $\Pi-\Pi$ interaction between carbon nanotube and benzene ring of nucleotides. ${ }^{213}$

\subsubsection{Mechanism of electro-oxidation}

The mechanism of electrochemical oxidation of guanine is well established. ${ }^{214,215}$ The oxidation involves two different steps. The first step is irreversible and involves loss of two electrons and two protons to form 8-hydroxyguanine. 8-hydroxyguanine further undergoes reversible oxidation step involving two electrons as shown in figure 17.

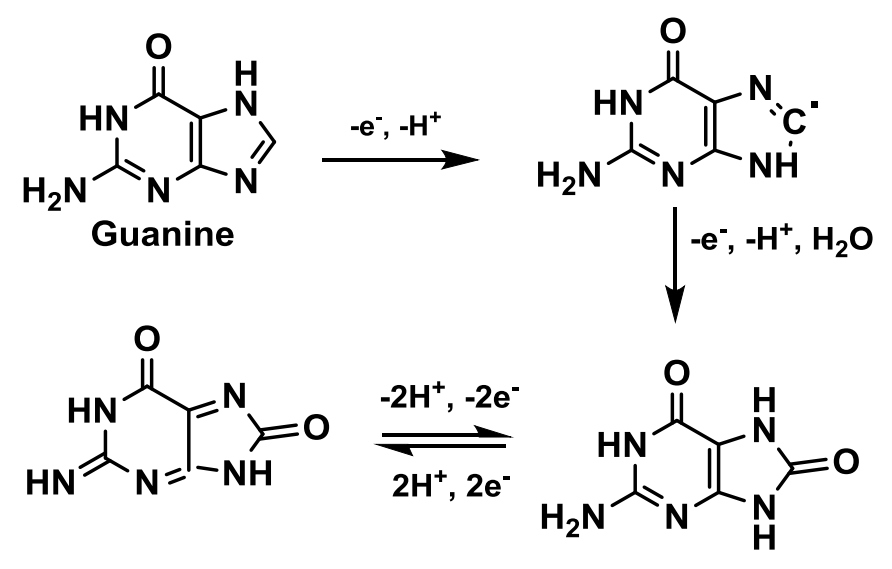

Figure 17. Electrochemical oxidation of guanine. Reaction mechanism, showing the formation of intermediates during the electrochemical oxidation of guanine.

The electro-oxidation of GMP and GTP also follows the similar path due to the same 
core structure of guanine. However, due to the presence of extra ribose sugar in GMP and GTP, we expect the oxidation occurs at different oxidation potentials.

\subsubsection{Optimization of conditions}

We optimize experimental parameters for the detection of guanine and its nucleotide.

\subsection{Surface density of P-SWCNT film electrode}

The surface density of P-SWCNT film electrode affects the kinetics of electrochemical oxidation of guanine and its nucleotides. It changes the conductivity as well as the number of adsorption sites for guanine on the film surface. To find out the optimum surface density of P-SWCNT, we varied the surface density from 1.1 to 11.0 $\mu \mathrm{gcm}^{-2}$.

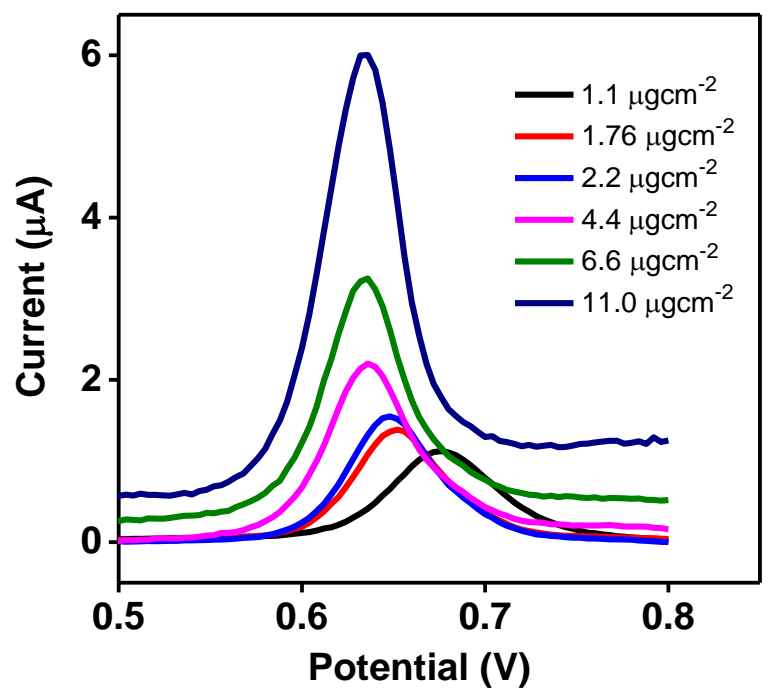

Figure 18. Effect of surface density of P-SWCNT film electrodes on electrochemical oxidation of guanine. Differential pulse voltammograms (DPV) of paper-based PSWCNT film electrodes with densities from 1.1 to $11 \mu \mathrm{gcm}^{-2}$ for the oxidation of $20 \mu \mathrm{M}$ of guanine in $0.1 \mathrm{M}$ phosphate buffer at $\mathrm{pH} 7.0$.

Figure 18 represents the background subtracted DPV of $20 \mu \mathrm{M}$ of guanine on the paper-based P-SWCNT film electrode. We found that surface density affects the peak potential, peak current as well as background current, indicating the electrocatalytic 
response of the paper-based P-SWCNT film electrode for guanine highly depends on the surface density. Both background current, as well as oxidation peak current, increases with the increase of surface density of P-SWCNT. However, peak potential decreases from $\sim 0.68 \mathrm{~V}$ to $\sim 0.64 \mathrm{~V}$ as shown in figure 18 .

To confirm whether the effect of P-SWCNT density is similar for oxidation of guanine nucleotides, we also studied the oxidation of GMP with the paper-based P-SWCNT film electrode prepared with variable surface density from $1.1 \mathrm{\mu gcm}^{-2}$ to $16.6 \mathrm{\mu gcm}^{-2}$. Figure 19A represents the variation in peak potential with the surface density of P-SWCNT. The peak potential decreases from $\sim 0.95 \mathrm{~V}$ to $\sim 0.88 \mathrm{~V}$ with an increase in surface density from $1.1 \mathrm{\mu gcm}^{-2}$ to $6.6 \mathrm{\mu gcm}^{-2}$. No further decrease in peak potential was observed with the surface density of P-SWCNT above $16.6 \mu \mathrm{gcm}^{-2}$.
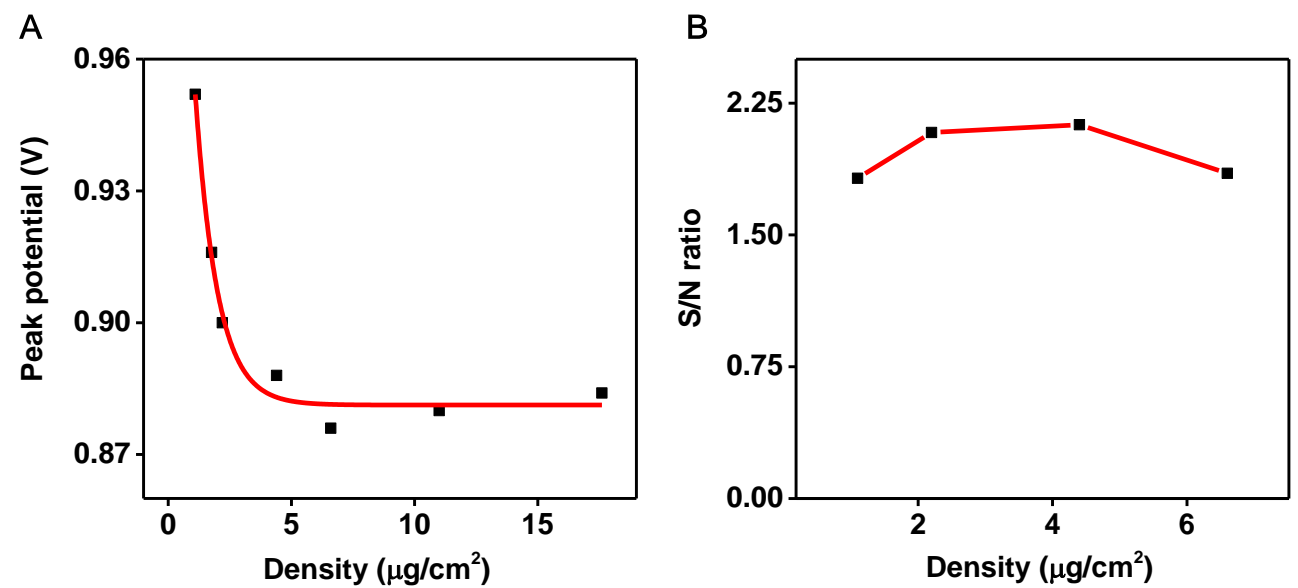

Figure 19. Effect of surface density on electrochemical oxidation of guanine nucleotides using the paper-based P-SWCNT film electrodes. (A) Variation of peak potential and (B) signal to noise ratio as a function of surface density of P-SWCNT for the oxidation of 20 $\mu \mathrm{M}$ of GMP in $0.1 \mathrm{M}$ phosphate buffer at $\mathrm{pH} 7.0$.

We expect the increase in $\mathrm{S} / \mathrm{N}$ ratio with the decrease of surface density as a result of lower background current of low-density P-SWCNT film electrodes. However, we found that $\mathrm{S} / \mathrm{N}$ ratio did not increase significantly when SWCNT density is higher than 
$6.6 \mu \mathrm{gcm}^{-2}$ (Figure19B). To balance the lower peak potential and higher S/N ratio, the PSWCNT film electrode prepared with $6.6 \mu \mathrm{gcm}^{-2}$ was chosen as optimized surface density for further experiments.

\subsection{Effect of $\mathrm{pH}$}

To study the effect of $\mathrm{pH}$ on electrochemical oxidation of guanine and its nucleotides, we changed the buffer $(0.1 \mathrm{M}$ acetate buffer for $\mathrm{pH} 3$ and 5 and $0.1 \mathrm{M}$ phosphate buffer for $\mathrm{pH} 7.0) \mathrm{pH}$ from 3 to 7 . Figure 20A shows background-subtracted DPV of paperbased P-SWCNT film electrodes for the oxidation of guanine at different $\mathrm{pH}$.

A

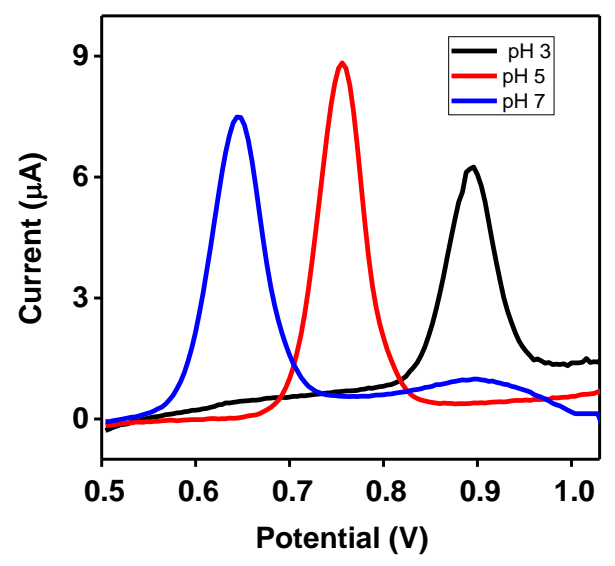

B

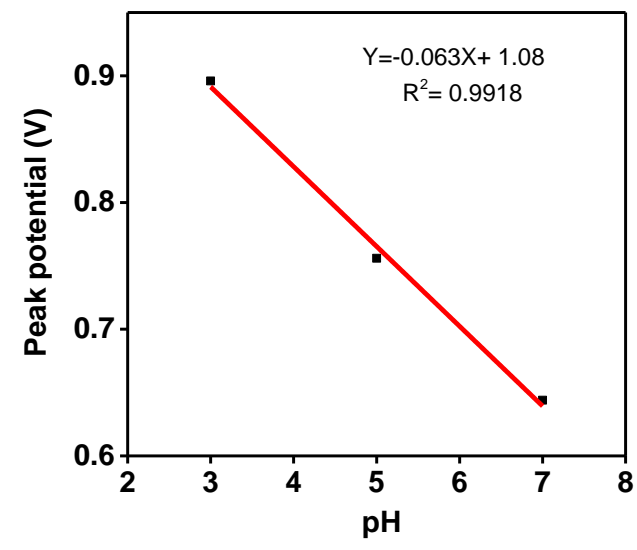

Figure 20. Effect of $\mathrm{pH}$ on electrochemical oxidation of guanine using paper-based $\mathrm{P}$ SWCNT film electrode. (A) Background-subtracted DPV of guanine oxidation using paper-based P-SWCNT film electrode at different $\mathrm{pH}$ and (B) the variation of peak potential and peak current as a function of $\mathrm{pH}$.

The oxidation peak potential decreases from $0.88 \mathrm{~V}$ to $0.64 \mathrm{~V}$ with the increase of $\mathrm{pH}$ from 3 to 7 . The plot of peak potential against $\mathrm{pH}$ gives the slope of $61 \mathrm{mV} / \mathrm{pH}$ which is close to the theoretical value of $59 \mathrm{mV} / \mathrm{pH}$, suggesting that electrochemical oxidation of guanine is governed by changing two hydrogen ions. However, maximum peak current was found in $\mathrm{pH}$ 5.0. To mimic the physiological $\mathrm{pH}$ and use relatively low peak 
potential, the $0.1 \mathrm{M}$ phosphate buffer $(\mathrm{pH} 7)$ was chosen for further experiments.

\subsubsection{Detection of Guanine (G)}

Differential pulse voltammetry (DPV) was used for the detection of guanine in the concentration range of $1 \mu \mathrm{M}$ to $500 \mu \mathrm{M}$. A distinct peak was observed at potential of 0.64 $\mathrm{V}$ (Figure 21A), indicating the electrocatalytic response of guanine on paper-based SWCNT film electrode.

A

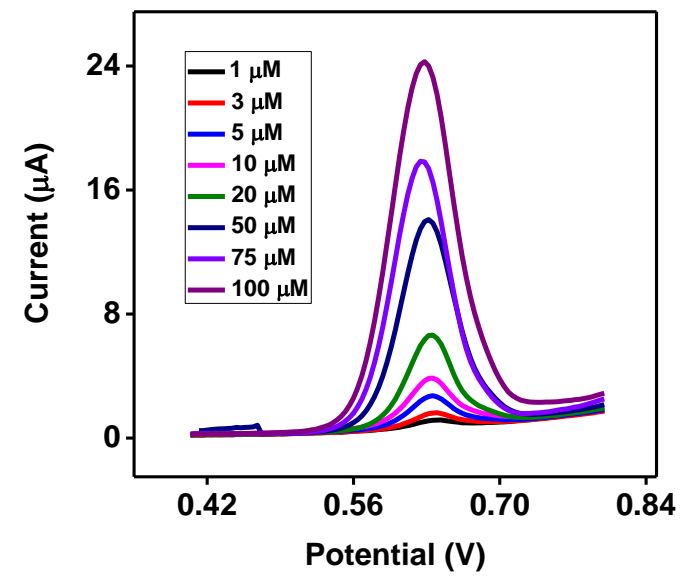

B

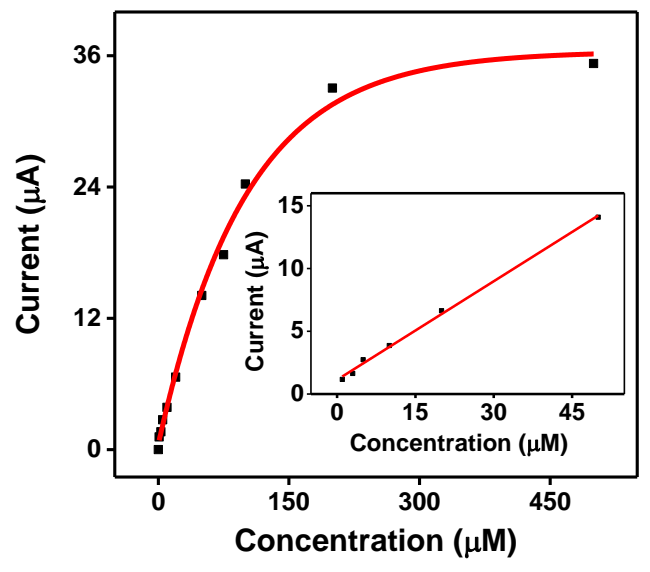

Figure 21. Electrochemical detection of guanine on the paper-based P-SWCNT film electrode. (A) Background-subtracted DVP of various concentrations of guanine. (B) A plot of peak current against the guanine concentration. Inset: linear range. Experiment was performed in $0.1 \mathrm{M}$ phosphate buffer $(\mathrm{pH} 7.0)$.

The peak potential is at least about $35 \mathrm{mV}$ less positive than previously reported values with different CNT-modified electrodes at pH 7.0 (Table1). The peak current increases with the increase of guanine concentration and reaches saturated after concentration of $200 \mu \mathrm{M}$ (Figure 21B). The calibration curve was obtained with a linear range of 1 to $20 \mu \mathrm{M}$ (Figure 21B). The linear regression equation was found to be $\mathrm{I}(\mu \mathrm{A})$ $=0.228 \mathrm{C}+1.47(\mathrm{r}=0.997)$. The calculated limit of detection is determined to be 0.06 $\mu \mathrm{M}$ based on the signal to noise ratio of 3 . Clearly, the detection limit is comparable to 
previously reported SWCNT/MWCNT modified electrodes except few ionic liquid/CNT modified electrodes as shown in Table 1.

Table 1. Performance Comparison of various CNT-modified electrodes for Guanine detection.

\begin{tabular}{|c|c|c|c|c|c|}
\hline Electrode & Method & $\begin{array}{l}\text { Peak } \\
\text { potential } \\
\text { (V) }\end{array}$ & LOD $(\mu M)$ & $\begin{array}{l}\text { Linear } \\
\text { range } \\
(\mu \mathrm{M})\end{array}$ & References \\
\hline MWCNT/Cholin/GCE & DPV & & 0.06 & $0.20-450$ & Wang et al. ${ }^{216}$ \\
\hline $\begin{array}{l}\mathrm{PbO}_{2} / \mathrm{MWCNT} / \mathrm{RTIL} / \mathrm{GC} \\
\mathrm{E}\end{array}$ & DPV & & 0.006 & $0.07-20$ & Liu et al. ${ }^{84}$ \\
\hline MWCNT-OH/GCE & LSV & & 0.02 & $0.2-10.0$ & Tu et al. ${ }^{211}$ \\
\hline MWCNT/PNF/GCE & DPV & 0.707 & 95.7 & $100-850$ & Tang el al. ${ }^{85}$ \\
\hline SWCNT/EPPGE & SWV & & 0.05 & $0.1-2.0$ & Goyal el al. ${ }^{45}$ \\
\hline$\beta$-SWCNT/GCE & DPV & & 0.01 & $0.5-12.0$ & Deng et al. ${ }^{18}$ \\
\hline$\beta$-CD/MWCNT/GCE & DPV & & 0.0337 & $10-200$ & Shen et al. ${ }^{86}$ \\
\hline $\begin{array}{l}\text { PTH/AuNP/MWCNT/GC } \\
\text { E }\end{array}$ & CV & 0.7 & 0.01 & $0.05-5$ & Liu et al. ${ }^{88}$ \\
\hline$\beta$-CD/MWCNT/graphite & DPV & 0.79 & 0.20 & $0-20$ & Wang et $a l^{218}$ \\
\hline IL/MWCNT/AuNP/GCE & CV & 0.73 & 0.005 & $0.008-2$ & Xiao et al. ${ }^{82}$ \\
\hline fMWCNT/AuNP/HPBCD & $\mathrm{CV}$ & 0.703 & 90 & $360-1200$ & Umasankar et al. ${ }^{8 t}$ \\
\hline P-SWCNT paper & DPV & 0.645 & 0.06 & $0-20$ & This work \\
\hline
\end{tabular}

\subsubsection{Detection of Guanosine monophosphate (GMP)}

Differential pulse voltammetry (DPV) was also used for the detection of GMP in the concentration range of $1 \mu \mathrm{M}$ to $500 \mu \mathrm{M}$. A well-defined peak was observed at the potential of $0.88 \mathrm{~V}$ as shown in figure $22 \mathrm{~A}$.

The peak potential is about $0.1 \mathrm{~V}$ less positive than previously reported values obtained with different electrodes at $\mathrm{pH} 7.0$ (Table 2). It demonstrated electrocatalytic activity of paper-based P-SWCNT film electrode for oxidation of GMP. The peak current 
increases with the increase of the concentration of GMP and reaches saturated at the concentration of $1000 \mu \mathrm{M}$. A calibration curve is obtained with a linear range of 3 to 100 $\mu \mathrm{M}$ as shown in figure 22B. The linear regression equation is found to be $\mathrm{I}(\mu \mathrm{A})=0.104$ $\mathrm{C}+2.74(\mathrm{r}=0.997)$. The limit of detection was calculated to be $0.245 \mu \mathrm{M}$ based on the $\mathrm{S} / \mathrm{N}$ ratio of 3 .

A

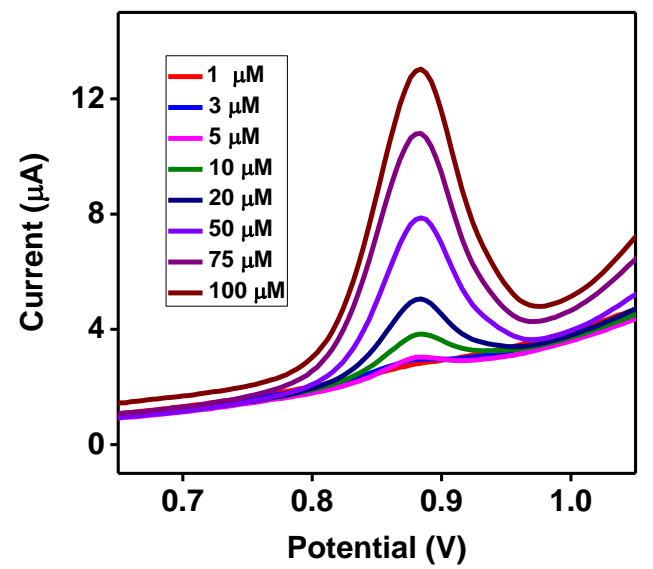

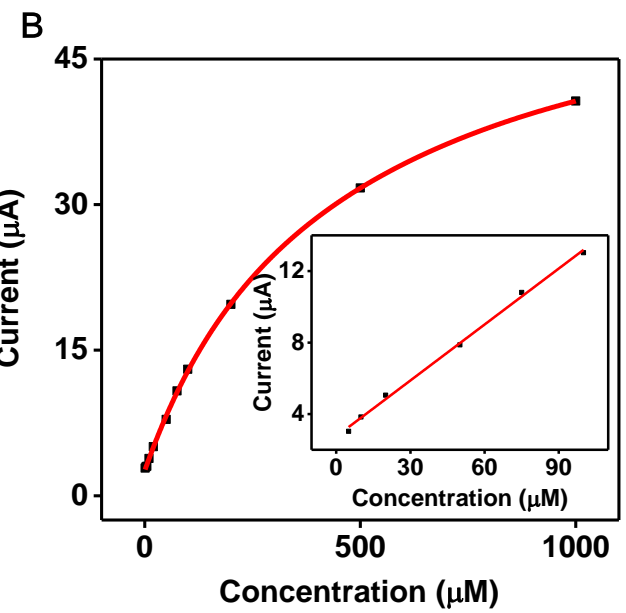

Figure 22. Electrochemical detection of GMP using paper-based P-SWCNT film electrode. (A) Background-subtracted DVP of various concentrations of GMP. (B) A plot of peak current against the concentration showing linear range. Experiment was performed in $0.1 \mathrm{M}$ phosphate buffer $(\mathrm{pH} 7.0)$.

Table 2. Comparison of different carbon-based electrodes for GMP detection

\begin{tabular}{|c|c|c|c|c|c|}
\hline Electrode & Method & $\begin{array}{l}\text { Peak } \\
\text { potential(V) }\end{array}$ & $\begin{array}{l}\text { LOD } \\
(\mu \mathrm{M})\end{array}$ & $\begin{array}{l}\text { Linear } \\
\text { range }(\mu \mathrm{M})\end{array}$ & References \\
\hline Graphene/MWCNT/GCE & Amperometry & 0.987 & 0.025 & 0.1 to 59.7 & ${\text { Huanshun et } a l^{219}}^{219}$ \\
\hline Nanocarbon/treated & SWV & 1.10 & - & - & Kato et al..$^{220}$ \\
\hline GCE (pre-conditioning) & DPV & $0.89(7.4)$ & - & - & Oliveira-Brett et al. ${ }^{221}$ \\
\hline Pyrrolic Graphite & & 1.1 & - & - & Goyal et.al. ${ }^{222}$ \\
\hline P-SWCNT paper & DPV & 0.88 & 0.245 & 3 to 100 & This work \\
\hline
\end{tabular}


Table 2 shows the performance comparison of various carbon-based electrodes for the electrochemical oxidation of GMP. When compared with these previously reported results, our paper-based P-SWCNT film electrode shows at least $0.1 \mathrm{~V}$ positive peak potential showing electrocatalytic activity towards the oxidation of GMP. Except for hybrid graphene and MWCNT-modified GC electrode, the P-SWCNT paper demonstrated a lower LOD of $0.245 \mu \mathrm{M}$ and a higher dynamic range of $3 \mu \mathrm{M}$ to $100 \mu \mathrm{M}$.

\subsubsection{Detection of Guanosine triphosphate (GTP)}

Differential pulse voltammetry (DPV) was also used for the detection of GTP in the concentration range of $3 \mu \mathrm{M}$ to $500 \mu \mathrm{M}$. A well-defined peak was observed at the potential of $0.872 \mathrm{~V}$ as shown in Figure 23A. The oxidation potential of GTP is almost similar to GMP, indicating there is a negligible effect of the phosphate group on oxidation of guanine.

A

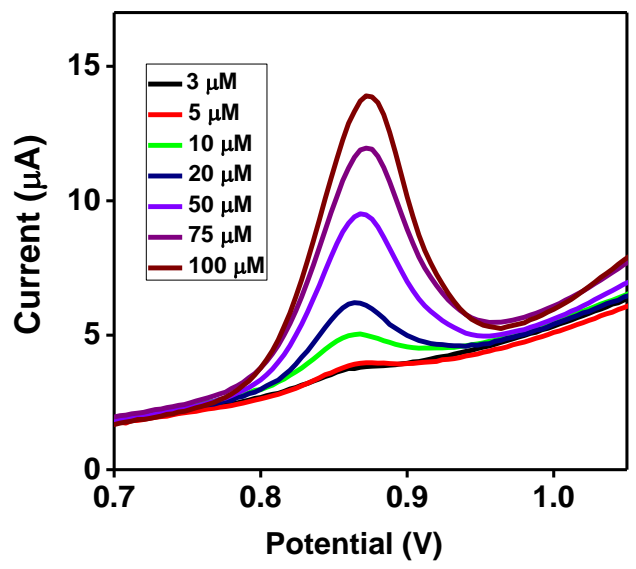

B

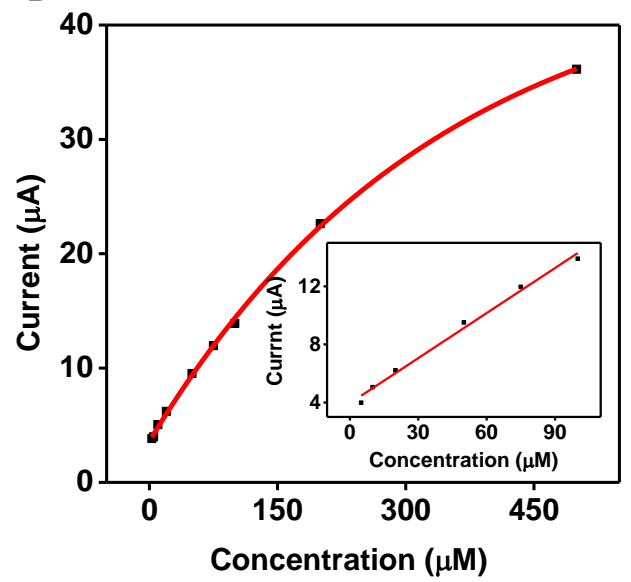

Figure 23. Electrochemical detection of GTP on the paper-based P-SWCNT film electrode. (A) Background-subtracted DVP of various concentrations of GTP. (B) A plot of peak current against the concentration of GTP showing a linear range between 3 to $100 \mu \mathrm{M}$. Experiment was performed in 0.1M PBS (pH 7.0). 
The peak current increases with the increase of the concentration of GTP and reaches saturated at $500 \mu \mathrm{M}$ as shown in Figure 23B. A calibration curve was obtained with a linear range of 3 to $100 \mu \mathrm{M}$ and with linear regression equation, I $(\mu \mathrm{A})=0.1035 \mathrm{C}$ $+3.94(r=0.995)$. The detection limit was calculated to be $0.3 \mu \mathrm{M}$ based on $\mathrm{S} / \mathrm{N}$ ratio of

3. This is the first report showing successful electrochemical oxidation of GTP.

\subsection{Broader Impacts}

We have shown that paper-based P-SWCNT thin film fabricated by routine vacuum filtration method can directly use as disposable electrodes for electrochemical sensing applications. This porous, uniform, well-dispersed, conductive CNT network on the paper substrate provides an excellent alternative of traditional CNT-modified electrodes. As compared to previously used CNT-modified electrodes, the paper-based P-SWCNT thin film is not only is simple and low-cost but also have comparable or even better performance as demonstrated by electrochemical detection of guanine and its nucleotides. Given the electrocatalytic activity of CNT for many electroactive molecules, the paper-based CNT electrode can be used as a general electrode material for electrochemical detection. The paper-based electrochemical detection platform could be incorporated into paper-based analytical devices for sensitive detection of target analytes. We envision that the paper-based CNT electrode could lead to the development of low-cost, portable and disposable analytical devices for on-site or point of care detection of target analytes. 
Chapter 4. A paper-based porous, three-dimensional Pt-CNT hybrid film electrode for methanol oxidation

\subsection{Introduction}

Platinum is a single best-known metal catalyst for a wide range of chemical reactions such as methanol oxidation, ethanol oxidation, and hydrogen iodide decomposition. ${ }^{94,223}$ It also has been widely used as an electrocatalyst for sensing, ${ }^{224}$ fuel cells, ${ }^{4,5}$ and solar cells ${ }^{96}$ applications. The use of platinum for such applications often requires the formation of platinum nanoparticles (PtNPs) or its clusters directly on different conducting supports such as glassy carbon electrode (GCE), ${ }^{225}$ fluorine-doped tin oxide $(\text { FTO })^{226}$ and carbon paper. ${ }^{227}$ Usually, the choice of electrode surfaces and platinum structures is aimed at achieving simple, effective, fast and low-cost platinum electrocatalyst.

PtNPs can be formed on the surface of conducting supports such as multi -walled carbon nanotube (MWCNT), ${ }^{95}$ single-walled carbon nanotube (SWCNT), ${ }^{228}$ carbon black, ${ }^{229}$ carbon nanofibers, ${ }^{101}$ activated carbon ${ }^{230}$ or graphene. ${ }^{231}$ Among them, SWCNT is considered as nearly ideal conducting support for forming platinum catalysts mainly due to its high surface area to disperse PtNPs, good electrical conductivity for faster heterogeneous electron transfer and higher corrosion resistance due to its inertness..$^{91,20,92}$ Therefore, different methods have been developed to fabricate PtSWCNT hybrid material with minimally damaged CNT structure and high surface area to volume ratio of Pt nanostructures.

The most common is solution-based methods where pre-formed PtNP-CNT material was mixed with $5 \%$ Nafion solution before casting on the electrode surface. In most widely used impregnation-reduction method, PtNPs were covalently linked with functionalized CNT by in situ reduction of precursor salt. First, CNT was functionalized 
either by chemical oxidation with nitric acid or by dispersion with surfactants such as sodium dodecyl sulfate (SDS), ${ }^{99}$ polystyrene-alt-maleic acid (PSMA) ${ }^{100}$ or with polymers such as polyvinylpyrrolidone (PVP), ${ }^{101}$ or with ionic liquids ${ }^{98}$ or with other compounds such as 1 - aminopyrene, ${ }^{102}$ perylene tetracarboxylic acid. ${ }^{103}$ The functionalized CNT was then separated from rest of the products by washing and centrifugation steps. Before the reduction, the functionalized CNT was re-dispersed in suitable solvents (glycerol, ${ }^{104}$ ethylene glycol, ${ }^{105}$ mixture of ethylene glycol and acetone ${ }^{106}$ or 1 -propanol ${ }^{95}$ ) containing precursor salt, reducing agents in the presence or absence of stabilizing agents (sodium citrate). The reduction was then carried out at a higher temperature of $100^{\circ} \mathrm{C}-150^{\circ} \mathrm{C}$ or with the aid of microwave using suitable reducing agent. The formed Pt-SWCNT composite is purified by multiple washing and centrifugation cycles and washed with water and dried at $60^{\circ} \mathrm{C}-70^{\circ} \mathrm{C}$ under vacuum.

As an alternative, the colloidal method has been used where PtNPs were synthesized separately (citrate-capped, ${ }^{92} \mathrm{PPh}_{3}$ modified, ${ }^{113}$ ethylene glycol dispersed $^{91,114}$ ) by reducing its precursor salt in the presence of stabilizing agents or suitable solvents. The pre-synthesized PtNPs were then attached on the surface of CNT either by mechanically mixing, or by $\mathrm{pH}$-induced adsorption or by using linkers or polymers or ionic liquids to form PtNP-SWCNT hybrid material. The flexibility of this method have been used to prepare highly dispersed, shape and facet-controlled platinum-CNT composites. ${ }^{105}$ However, adsorption of impurities on the Pt surface and formation of embedded PtNP structures significantly reduces the catalytic efficiency. Furthermore, most commonly used solvents (ethylene glycol, glycerol) have high viscosity and a high boiling point which causes difficulty during filtering and rinsing steps of fabrication and hard to completely remove solvent from the Pt surface. 
In contrast to solution-based methods, pure PtNPs were directly formed on the external accessible surface of SWCNT thin films for better catalytic efficiency by surface deposition methods such as electrochemical deposition, ${ }^{121}$ or electrodeless deposition. ${ }^{126}$ These methods involved cleaner reduction of Pt precursor on the surface of CNT thin film by electrochemical or galvanic force. So far, platinum has been electrodeposited on several CNT thin films fabricated by different routes such as direct growth of $\mathrm{CNT}$ on $\mathrm{SiO}_{2}{ }^{127}$ followed by electrochemical oxidation, or casting of functionalized CNT on GC, or by electrophoretic deposition of functionalized SWCNT on FTO, or by vacuum filtration of CNT suspensions on paper substrate followed by subsequent transfer into the surface of $\mathrm{GC}^{129}$ or carbon fiber ${ }^{130}$ or self-standing thick film of CNT as a buckypaper electrode. ${ }^{131}$ Despite the readily accessible pure PtNP deposits, the overall performance of these surface-based methods is not good due to lack of formation of $\mathrm{Pt}$ nanostructures that can maximize the surface area to volume ratio. To address the problem, the favorable hybrid materials have been achieved either by decreasing the size of spherical PtNPs or by forming different shaped PtNPs or interconnected porous platinum structures.

There is a great need for forming clean and catalytic PtNPs on the surface of CNT thin film with favorable PtNP structure. We here demonstrated the fabrication of interconnected, three-dimension (3D), porous and relatively clean PtNPs on the surface of SWCNT thin film using vacuum filtration technique. We first prepared SWCNT thin film on a porous membrane and then loaded pre-synthesized PtNPs on its top using the downward convective force of vacuum filtration. The porous nature of SWCNT thin film facilitates the removal of the aqueous phase from PtNP solution forming a conductive layer of PtNP/CNT hybrid film. The developed hybrid material has both favorable PtNP as well as pristine SWCNT structure. The porous microstructure SWCNT network 
maximizes both electron and the mass transfer from the surface of the electrode. The SWCNT under layer acts as both rigid support for PtNPs and conducting electrode material. Therefore, the resulting PtNP/SWCNT composite material can directly use as a flexible electrocatalyst without a need of transfer onto different electrode surfaces.

Furthermore, the paper-based PtNP/SWCNT hybrid film has several advantages that could not be achieved with traditional electrode materials such as glassy carbon, fluorine doped tin oxide, graphite: 1) The use of paper instead of electrode material leads to significant reduction of cost of electrode material; 2) It eliminates the requirement of pretreatment or cleaning of electrode surface before modification; 3) It eliminates the requirement of binding polymers to incorporate CNT on paper surfaces; 4) The prepared nanocomposite can directly use as a disposable electrode material or can easily transfer into preferable substrates which otherwise is technologically challenging; ${ }^{128} 5$ ) It is environment-friendly.

To demonstrate the superiority of the hybrid film prepared by vacuum filtration method, PtNPs were also deposited on the surface of SWCNT thin film using electrodeposition method. The electrocatalytic activity of the prepared PtNP/SWCNT hybrid composite was evaluated by oxidation of a benchmark molecule (methanol). The platinum microstructures formed on SWCNT-coated paper were characterized by cyclic voltammetry (CV), scanning electron microscope (SEM), X-ray photoelectron spectroscopy (XPS) and X-ray diffraction (XRD) analysis. Experimental results demonstrated that the PtNP/SWCNT hybrid film prepared using vacuum filtration produced a higher electrocatalytic behavior and the higher degree of resistance to carbon monoxide poisoning during methanol oxidation in the comparison with the hybrid film prepared using electrodeposition. 


\subsection{Experimental Approach}

Chemicals and materials. Mixed nitrocellulose membrane (MF-Millipore Membrane Filter, USA) was purchased from Millipore Corporation (Billerica, MA, USA). All other chemical reagents were purchased from Sigma-Aldrich and used without further purification. The aqueous solutions were prepared using deionized water from a Milli-Q water purification system (Millipore Corporation, Billerica, MA, USA).

Preparation of paper-based SWCNT thin film. SWCNT thin film was prepared on filter paper using reported vacuum filtration method. ${ }^{207}$ Briefly, the concentrated pure single-walled carbon nanotube (P-SWCNT)(stock concentration: $0.25 \mathrm{mg} / \mathrm{mL}$ ) from Nano Integris (prepared by Arc discharge method) was diluted in 1\% sodium dodecyl sulfate (SDS) solution and dispersed using sonication $(335 \mathrm{~W}, 50 / 60 \mathrm{~Hz})$ for 10 minutes. Welldispersed P-SWCNT solution was then poured onto nitrocellulose membrane (diameter: $0.1 \mu \mathrm{m})$ placed in vacuum filtration apparatus. For optimum uniformity, the solution was first sat for 5 minutes before applying vacuum. The vacuum was then applied slowly for 60 minutes to form P-SWCNT interconnected network on the membrane surface. The remaining surfactant was washed away using an excess amount of deionized water. The film was vacuum-dried overnight before making an electrode.

Fabrication of paper-based SWCNT thin film electrodes. A thin P-SWCNT film was used to make 24 working electrodes by cutting thin film into pieces of $0.3 \mathrm{~cm} \times 0.6$ cm size. Silver paint (TED PELLA) was used as a connector between copper wire and P-SWCNT thin film. The silver paint and copper wire were sealed with para-film as an insulator. The para-film covering was adjusted in such a way that it defines the geometrical surface area of working electrode as exactly $0.3 \mathrm{~cm} \times 0.4 \mathrm{~cm}$.

Electrochemical deposition of PtNPs on paper-based SWCNT thin film electrode. The electrochemical deposition of Pt nanoclusters on the SWCNT-loaded 
paper was carried out at constant potential of $-0.20 \mathrm{~V}$ for $600 \mathrm{~s}$ in $0.5 \mathrm{M} \mathrm{H}_{2} \mathrm{SO}_{4}$ solution containing $2.0 \mathrm{mM} \mathrm{H}_{2} \mathrm{PtCl}_{6}$. In control experiments, similar working conditions were used for the electrodeposition of Pt nanoclusters on glassy carbon electrode (GCE) and Fluorine-doped tin oxide (FTO). Before use, GCE was sequentially polished with $1.0 \mu \mathrm{m}$, $0.3 \mu \mathrm{m}$, and $0.05 \mu \mathrm{m}$ alumina powder, respectively. The electrode was washed with ethanol and distilled water after each polishing for 10 minutes using ultrasonication.

Synthesis of PtNPs. Platinum nanoparticles (size $=\sim 29 \mathrm{~nm}$ ) were synthesized by the seed-mediated process according to the procedure reported by Nadja C. Bigall. ${ }^{232}$ The Seed of $\sim 5 \mathrm{~nm}$ was prepared first. Briefly, $3.6 \mathrm{~mL}$ of $0.2 \%$ solution of chloroplatinic acid hexahydrate $\left(\mathrm{H}_{2} \mathrm{PtCl}_{6} \cdot 6 \mathrm{H}_{2} \mathrm{O}\right)$ was added to $46.4 \mathrm{~mL}$ boiling water. After one minute, $1.1 \mathrm{~mL}$ of the solution containing $1 \%$ sodium citrate and $0.05 \%$ citric acid was added, followed half a minute later by a quick injection of $0.08 \% 0.55 \mathrm{~mL}$ freshly prepared sodium borohydride containing $1 \%$ sodium citrate and $0.05 \%$ citric acid. The solution was then cooled to room temperature with stirring. In order to prepare $29 \mathrm{~nm}$ size particles, $1 \mathrm{~mL}$ of platinum seed was added to $29 \mathrm{~mL}$ of deionized water at room temperature. Then, $45 \mu \mathrm{L}$ of $0.4 \mathrm{M}$ chloroplatinic acid solution was added followed by addition of $0.5 \mathrm{~mL}$ of a solution containing $1 \%$ sodium citrate and $1.25 \% \mathrm{~L}$-ascorbic acid. Under stirring, the temperature was slowly increased to the boiling point at $\sim 10{ }^{\circ} \mathrm{C} / \mathrm{min}$, with a total reaction time of $30 \mathrm{~min}$. The solution was then cooled to room temperature with stirring.

Preparation of PtNP/SWCNT hybrid film using vacuum filtration technique. SWCNT thin film was first prepared by vacuum filtration method mentioned above. Before loading PtNPs on the P-SWCNT film, $13.1 \mathrm{~mL}$ of as-prepared PtNPs were washed three times by precipitation in the centrifuge (7100 rpm for 30 minutes). In each step, the supernatant was exchanged with an equal amount of DI water to re-disperse 
the particles. The re-dispersed particles were mixed well and poured slowly from a side on pre-formed SWCNT thin film. After five minutes, the vacuum was applied slowly to remove water to form PtNP/SWCNT hybrid thin film. The film was dried overnight before use.

Determination of amount of PtNP by ICP/MS. The total amount of platinum deposited on SWCNT paper was determined by using ICP/MS (PerkinEImer ELAN DRC) experiments. The PtNP/SWCNT hybrid films prepared by both methods was cut into the same size as an electrode. The film was then digested in $1 \mathrm{~mL}$ aquaregia solution for $12 \mathrm{hrs}$. To ensure complete dissolution of PtNPs from the paper, the film was digested further for 3 hours each in two fresh $1 \mathrm{~mL}$ aquaregia solutions. The sample solution was further diluted $10 \times$ in $2 \%$ nitric acid before the ICP/MS experiment. The solutions were then analyzed for ${ }^{195} \mathrm{Pt}$ signal, and the signal is converted into platinum concentration using calibration curve of sample set of known platinum concentrations from 1 to 100 ppm. The parameters for ICPMS experiments are as follows: RF power 1350W, Torch gas flow rate $(\mathrm{Ar})=0.8 \mathrm{Lmin}^{-1}$, Isotopes for internal standardization $={ }^{89} \mathrm{Y}$.

Characterization of films. The surface morphology of electrochemical deposited and vacuum filtration fabricated Pt/SWCNT hybrid films were characterized by Field emission scanning electron microscope (FE-SEM), X-ray photoelectron spectroscopy (XPS) and X-ray diffraction (XRD). FE-SEM image was obtained on an S-4800 microanalyzer (Hitachi, Japan). XPS was carried out on an ESCALAB 250 spectrometer equipped with a monochromatic Al Ka X-ray source (Thermo Fisher Scientific Inc., U.K.). XRD measurement was performed on a D/MAX 2200 VPC diffractometer using Cu Ka radiation $(\lambda=0.154056 \mathrm{~nm})$ with a Ni filter (Rigaku, Japan). 
Electrochemical measurements of methanol oxidation. All the electrochemical experiments were carried out at ambient condition using $\mathrm{CHI}$ electrochemical station. Different CNT electrode with platinum and other control electrodes were used as working electrode. $\mathrm{Ag} / \mathrm{AgCl}$ and platinum wire were used as reference and counter electrode respectively.

\subsection{Results and Discussion}

\subsubsection{Fabrication of paper-based PtNP/SWCNT hybrid film by electrodeposition} technique.
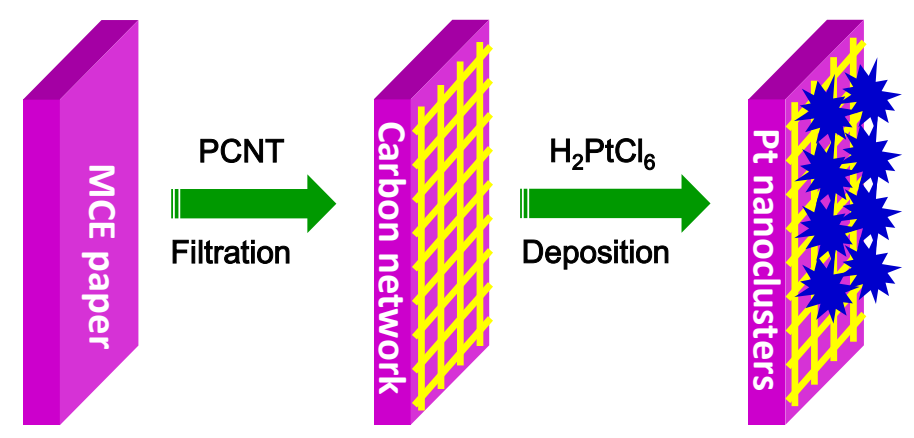

Figure 24. Schematic representation of formation of paper-based SWCNT thin film and deposition of PtNPs on the SWCNT film by electrodeposition method.

Figure 24 represents the schematic of fabrication of paper-based PtNP/SWCNT hybrid thin films by electrodeposition method. Specifically, we first prepared SWCNT thin film (density $=8.8 \mu \mathrm{g} / \mathrm{cm}^{2}$ ) by vacuum filtration method ${ }^{207}$ on mixed cellulose ester membrane. The method is well-established for the fabrication of thin, uniform layer of CNT on porous substrate. ${ }^{207}$ The well-dispersed CNT was trapped on porous membrane substrate (Figure 2B) by vacuum force resulting randomly oriented network of CNT on paper substrate. The formed P-SWCNT layer contains relatively well dispersed, and interconnected tube structure with overlapping of CNT. More importantly, it is mechanically rigid and conductive as a support structure for PtNPs. 


\subsubsection{Electrodeposition of PtNPs on the paper-based SWCNT thin film.}

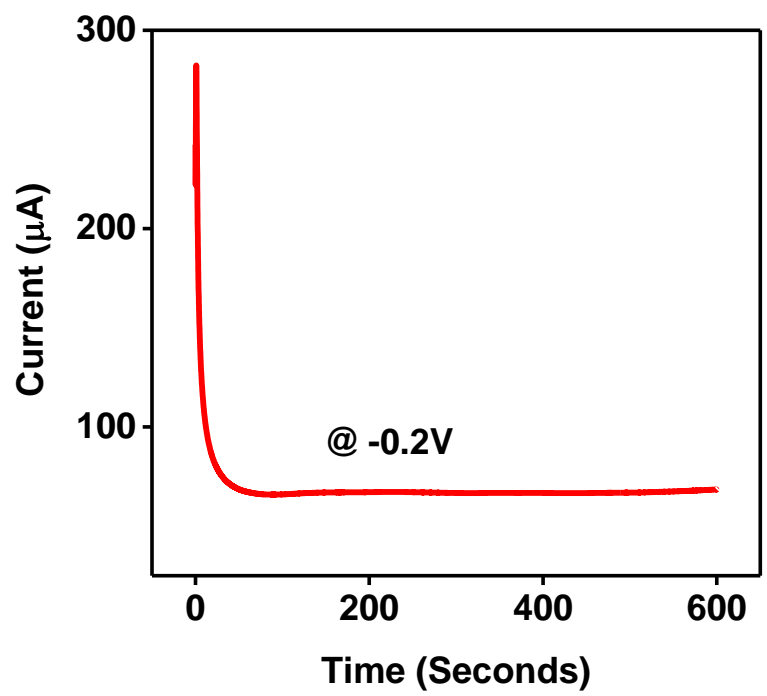

Figure 25. Current transients of SWCNT paper electrode during electrodeposition of platinum in $2.0 \mathrm{mM} \mathrm{H}_{2} \mathrm{PtCl}_{6}$ containing $0.5 \mathrm{M} \mathrm{H}_{2} \mathrm{SO}_{4}$. The deposition was carried out at $0.2 \mathrm{~V}$ for $600 \mathrm{~S}$.

To perform electrochemical deposition of platinum on the surface of paper-based SWCNT film, we first made paper-based SWCNT electrodes from the thin film (see procedure described above). The prepared electrode was directly used to deposit platinum from its precursor salt solution containing $2.0 \mathrm{mM} \mathrm{H}_{2} \mathrm{PtCl}_{6}$ in $0.5 \mathrm{M} \mathrm{H}_{2} \mathrm{SO}_{4}$. The potential and time were optimized as $-0.2 \mathrm{~V}$ for $600 \mathrm{~S}$ to obtain maximum deposition of PtNPs. Figure 25 represents the current transient curve for the electrochemical deposition of platinum on the SWCNT surface. The current transit shows typical behavior of P-SWCNT with initial steep decrease for about 20S followed by a slower decrease until 600S. This is due to initial oxidation of $\mathrm{Pt}^{\prime \prime}$ to $\mathrm{Pt}{ }^{\mathrm{IV}}$ followed by development of diffusion field and its interaction with the oxidation sites of Pt. ${ }^{122}$ 


\subsubsection{Characterization of the PtNP/SWCNT film prepared by electro-deposition.}

\subsubsection{Pt Concentration on the PtNP/SWCNT film by ICP-MS.}

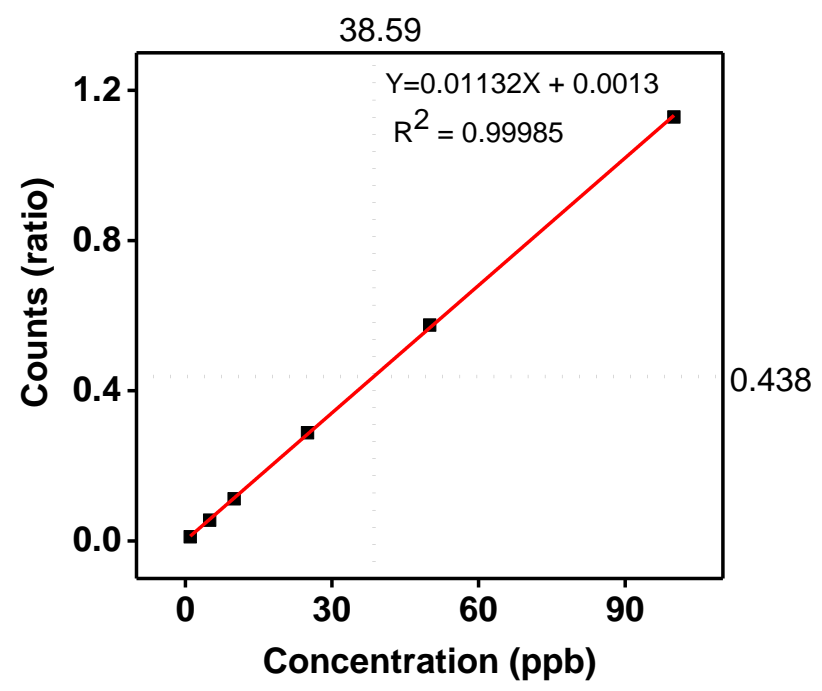

Figure 26. Calibration curve of standard ${ }^{95} \mathrm{Pt}$ for the determination of the amount of electrodeposited Pt from the surface of paper-based SWCNT thin film electrode. The Sample solution was prepared by dissolving Pt from the P-SWCNT surface using aquaregia solution.

The platinum from electrodeposited sample was extracted by incubating the electrode $\left(0.12 \mathrm{~cm}^{2}\right)$ in aqua regia for overnight. The extracted platinum was measured by ICP-MS and calculated with standard ${ }^{95} \mathrm{Pt}$ calculation curve. Figure 26 represents the calibration curve of standard ${ }^{95} \mathrm{Pt}$ in the range of 0 to $100 \mathrm{ppm}$ with the linear regression equation of $y=0.0113 x+0.0013\left(r^{2}=0.99985\right)$. The total amount of platinum per electrode was determined from ICP-MS experiments. We found that Pt density on the paper-based P-SWCNT film is $73.6 \mu \mathrm{g} / \mathrm{cm}^{2}$.

\subsubsection{Morphology and size distributions}

Figure 27 represents the morphology of electrodeposited platinum structures on paper-based P-SWCNT film. We observed that a porous feature in filter membrane (Figure 27A) and interconnected SWCNTs loaded on the paper (Figure 27B). Platinum 
was present in the form of nanoclusters having outer spike like tips on the surface, whichappeared as nano-flowers. The formation of such structure may be due to limited adsorption sites on hydrophobic surface of SWCNT. It has been reported that the adsorption sites control the nucleation and growth of platinum particles on CNT surface during electrodeposition that involves three steps: adsorption of platinum salt, formation of Pt complex and reduction of the Pt-complex to nanoparticle. ${ }^{122}$
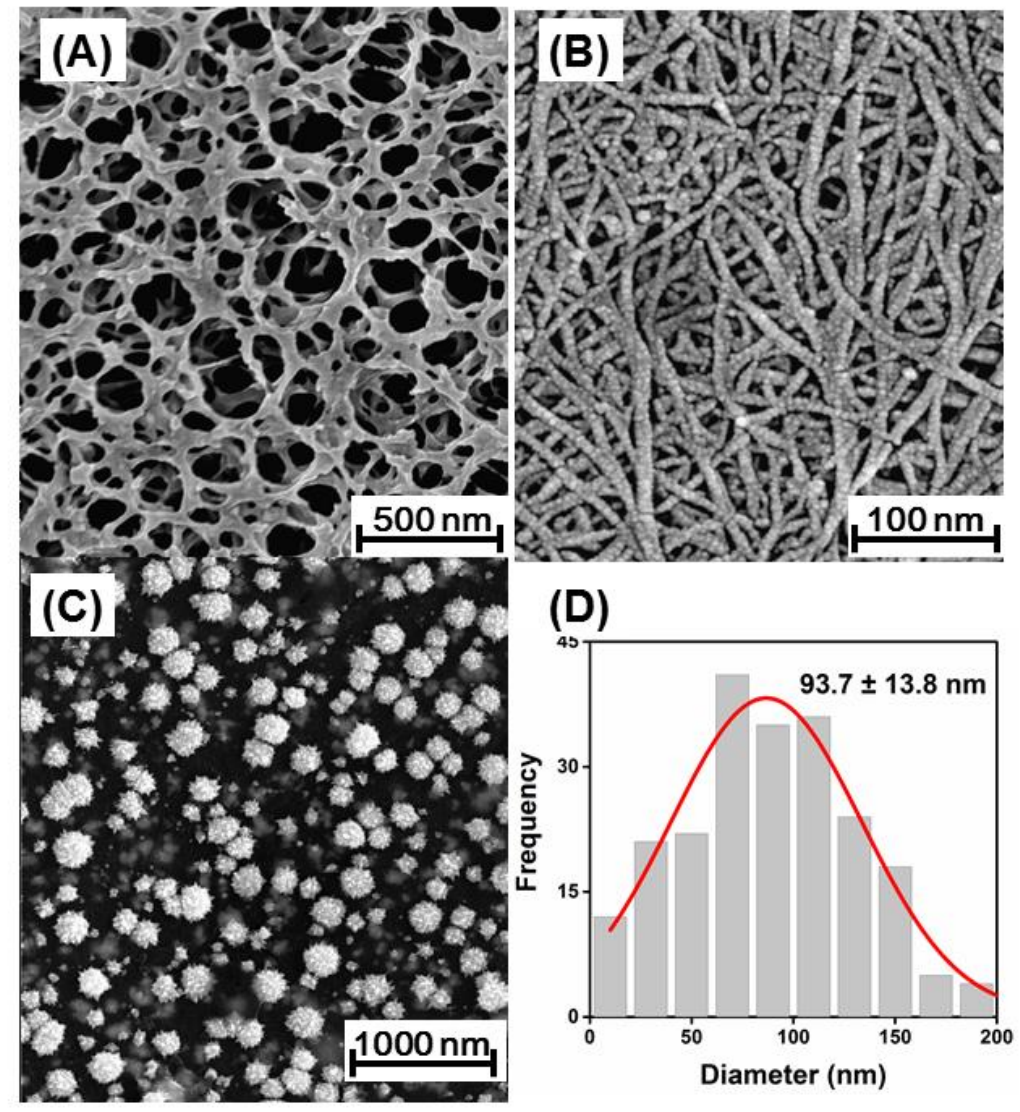

Figure 27. Morphology of various films. FE-SEM images of (A) Mixed cellulose ester filter paper (B) paper-based SWCNT thin films prepared by vacuum filtration method, (C) PtNPs on the SWCNT surface, and (D) size distribution of PtNPs for the paper-based PtNP/SWCNT film analyzed using imageJ software.

Therefore, it is reasonable to predict that nanoparticle formed during the electrodeposition acts as a site of nucleation for further growth of particle to form complex structure. The formation of nanoclusters instead of the continuous thin film is in 
accordance with the previous reports that nano-clusters ${ }^{124}$ are formed on the surface of CNT having very few or not functionalized groups. Statistical analysis of SEM image using imageJ software indicates that average size of platinum nano-flower like structures is $94 \pm 17 \mathrm{~nm}$ (Figure 27, C \& D).

\subsubsection{Platinum state of the paper-based PtNP/SWCNT thin film.}

X-ray photoelectron spectroscopy (XPS) was used to verify the formation of $\mathrm{Pt}$ structures and its oxidation state on paper-based P-SWCNT film. Figure 28A represents the XPS analysis of PtNP/SWCNT composites showing platinum $4 \mathrm{f}$ spectra, indicating the presence of platinum on the surface of the P-SWCNT film. The spectra are wellresolved showing doublets as $\mathrm{Pt}_{47 / 2}$ and $\mathrm{Pt}_{4} \mathrm{f}_{5 / 2}$ on PtNP/SWCNT film with binding energies of $71.63 \mathrm{eV}$ and $74.88 \mathrm{eV}$, respectively. A comparison with literature value suggests that binding energies corresponding to the $\mathrm{Pt}(0) .{ }^{233}$ The presence of a higher percentage of $\mathrm{Pt}(0)$ suggests higher catalytic efficiency could be achieved on the prepared PtNP/SWCNT film.

A

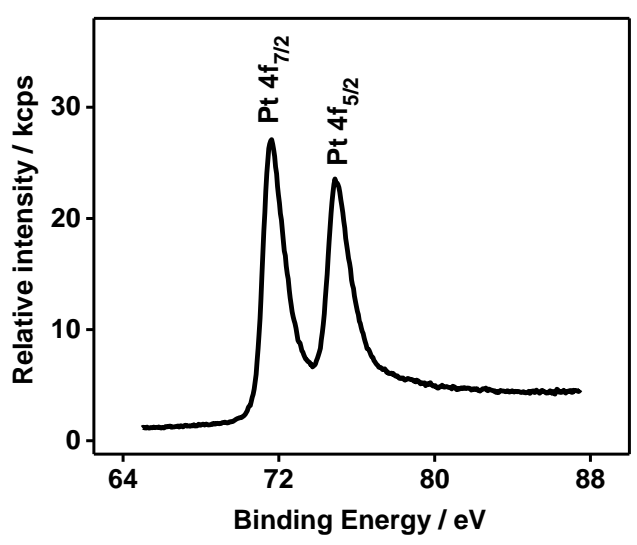

B

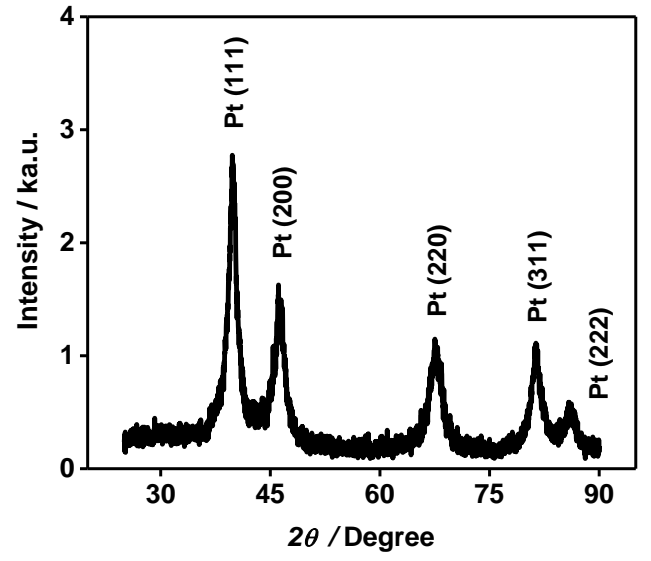

Figure 28. State of Pt on the surface of paper-based SWCNT thin film. (A) XPS spectra showing oxidation state of platinum and (B) XRD spectra showing the crystalline state of PtNPs on the surface of paper-based SWCNT film. 
The crystallinity of the platinum nanostructure was determined by X-ray diffractometry $(\mathrm{XRD})$. Figure $28 \mathrm{~B}$ represents the $\mathrm{XRD}$ pattern of prepared $\mathrm{Pt}$ nanoclusters on the surface of paper-based SWCNT film. The Peaks at $2 \theta=39.8^{\circ}$, $46.12^{\circ}, 67.52^{\circ}, 84.9^{\circ}$ and $87.04^{\circ}$ correspond to the crystalline planes of face-centered cubic as Pt (111), Pt (200), Pt (220), Pt (311) and Pt (222), respectively, indicating that platinum exists in crystalline form as in bulk $\mathrm{Pt}^{234}$

\subsubsection{Evaluation of paper-based PtNP/SWCNT hybrid film electrode for} electrochemical oxidation of methanol

We choose methanol as a benchmark molecule to evaluate the electrocatalytic performance of paper-based PtNP/SWCNT hybrid film electrode. Electrocatalytic activity towards methanol oxidation was determined by performing cyclic voltammetry experiments in $0.1 \mathrm{M} \mathrm{H}_{2} \mathrm{SO}_{4}$ containing $0.1 \mathrm{M}$ methanol at the scan rate of $50 \mathrm{mV} / \mathrm{s}$.

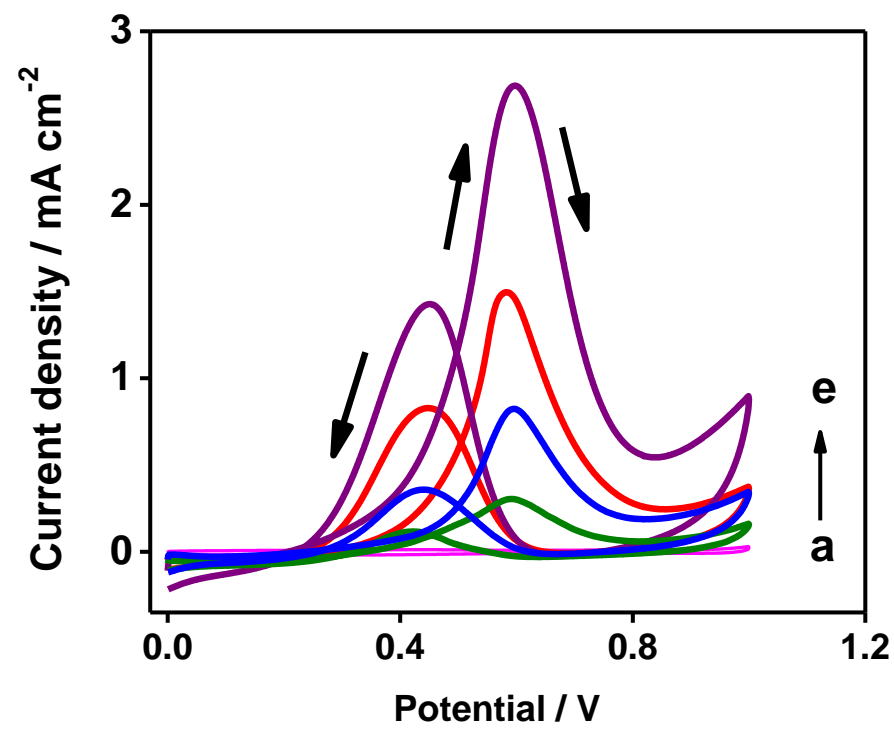

Figure 29. Evaluation of methanol oxidation on various electrodes. Cyclic voltammetry responses of (a) paper-based SWCNT film electrode, (b) commercially purchased $\mathrm{Pt}$ electrode, (c) PtNP-deposited GCE (d) PtNP-deposited FTO and (e) paper-based PtNP/SWCNT hybrid film electrode in $0.1 \mathrm{M} \mathrm{H}_{2} \mathrm{SO}_{4}$ containing $0.1 \mathrm{M}$ methanol at the scan rate of $50 \mathrm{mV} / \mathrm{s}$. All PtNP-modified electrodes are prepared by electrodeposition of $\mathrm{Pt}$ at constant potential of $-0.2 \mathrm{~V}$ for $600 \mathrm{~S}$. 
As a comparison, platinum was also electrochemically deposited on traditional electrode surfaces such as glassy carbon electrode (GCE) and fluorine-doped tin oxide (FTO) using similar experimental conditions.

It is evident from Figure 29 that except for only P-SWCNT film electrode, all the electrodes showed characteristic methanol oxidation peaks with forward peak potential at $\sim 0.65 \mathrm{~V}$ and backward peak potential at $\sim 0.44 \mathrm{~V}$. No methanol peak was observed for the paper-based P-SWCNT film electrode, indicating that SWCNT has no direct role in the oxidation of methanol. Very small oxidation peak current was obtained on commercially-purchased Pt electrode. The peak current densities on PtNPs deposited on GCE, FTO and P-SWCNT electrodes have $2 \times, 4 \times$, and $7 \times$ higher peak current density than the bulk platinum electrode.

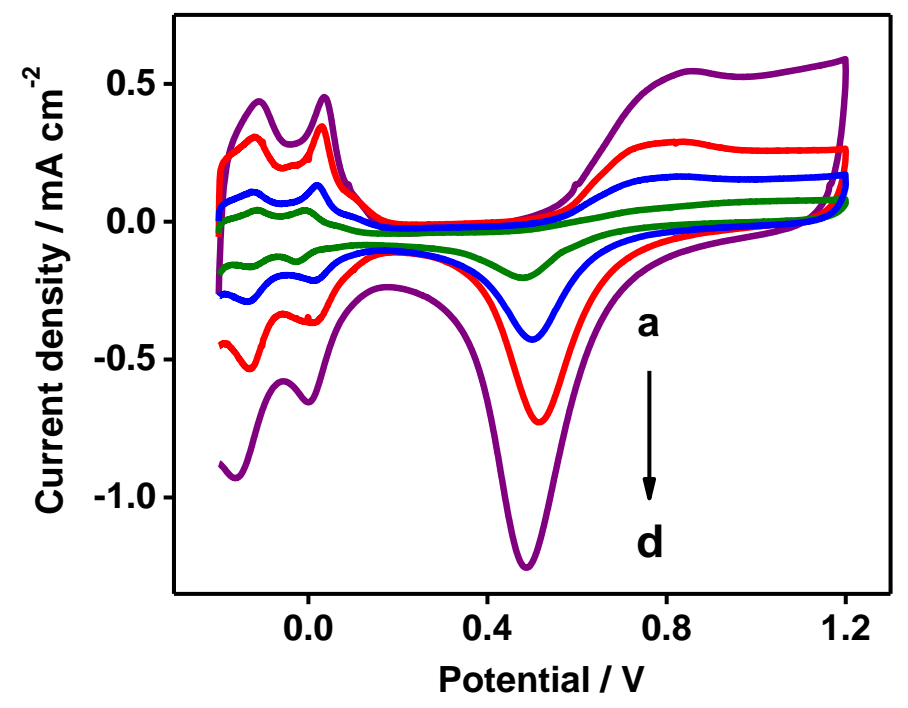

Figure 30. Electroactive surface area of different electrodes. Cyclic voltammetry responses of (a) commercially purchased Pt electrode, (b) PtNP-deposited GCE, (c) PtNP-deposited FTO, and (d) paper-based PtNP/SWCNT film paper in $0.5 \mathrm{M} \mathrm{H}_{2} \mathrm{SO}_{4}$ at the scan rate of $50 \mathrm{mVs}^{-1}$. All PtNP-deposited electrodes are prepared by electrodeposition of $\mathrm{Pt}$ from its precursor salt solution at a constant potential of $-0.2 \mathrm{~V}$ for $600 \mathrm{~S}$. 
The difference in the performance of platinum on various supporting electrode materials could be explained by measuring their electroactive surface area. Figure 30 represents the cyclic voltammetry curve of different electrodes in $0.5 \mathrm{M} \mathrm{H}_{2} \mathrm{SO}_{4}$, used to calculate the electroactive surface area by hydrogen adsorption method. ${ }^{235}$ The electroactive surface area of the PtNP-deposited electrode can be estimated from the equation: $A=Q_{H} / Q_{m}$ (where $Q_{H}$ is the charge of the hydrogen adsorption/desorption region of the $C V$, and $Q_{m}$ is the charge associated with the monolayer adsorption of hydrogen $\left(Q_{m}=210 \mu \mathrm{C} / \mathrm{cm}^{2}\right)$. Using this equation, we calculated that the electroactive surface area for commercially purchased Pt electrode, PtNP-deposited GCE, PtNPdeposited FTO, and paper-based PtNP/SWCNT film electrode were 0.040, 0.465, 0.152, and $1.12 \mathrm{~cm}^{2}$, respectively.

The roughness factor was determined by the following equation: $R_{\mathrm{f}}=A_{\mathrm{ed}} / A_{\mathrm{g}}$ (where $A_{\mathrm{ec}}$ is the electrochemical surface area of the PtNP-modified electrode, and $A_{\mathrm{g}}$ is the geometric surface area of electrode). Thus, the roughness for commercially purchased Pt electrode, PtNP-deposited GCE, PtNP-deposited FTO, and paper-based PtNP/SWCNT film electrode were calculated to be $1.27,2.15,5.16$, and 9.33, respectively. The experimental results demonstrated that the real surface area was increased up to $~ 7.35$ times in SWCNT film as compared to just $\sim 1.69 \times$ in GCE and 4.06x in FTO which indicates SWCNT paper provides a larger surface support for Pt deposition as compared to traditional electrode material with a solid surface.

\subsubsection{Fabrication of paper-based PtNP/SWCNT hybrid film using vacuum filtration technique.}

Although electrochemical method provides a simple, rapid and facile method to load PtNPs on the surface of CNT, it is not suitable for mass production and precise control of amount and size of the platinum structures. Layer-by-layer assembly by vacuum filtration 
has been used as a very simple and versatile method for incorporation of various nanomaterials without the use of a binder. The versatility of vacuum filtration allows the formation of various nanomaterial by downward convective force. ${ }^{236}$ The highly porous structure of CNT thin film, allows removal of surfactant solvent, theoretically, platinum nanoparticles of any size or shape can be deposited on top of porous CNT film. Based on this concept, we have demonstrated the deposition of citrate-capped gold nanoparticles atop of SWCNT thin film on paper substrate. ${ }^{57}$ We further extended the technique to deposit citrate-coated PtNPs on top of CNT film to prepare Pt/SWCNT hybrid thin film on the paper substrate.

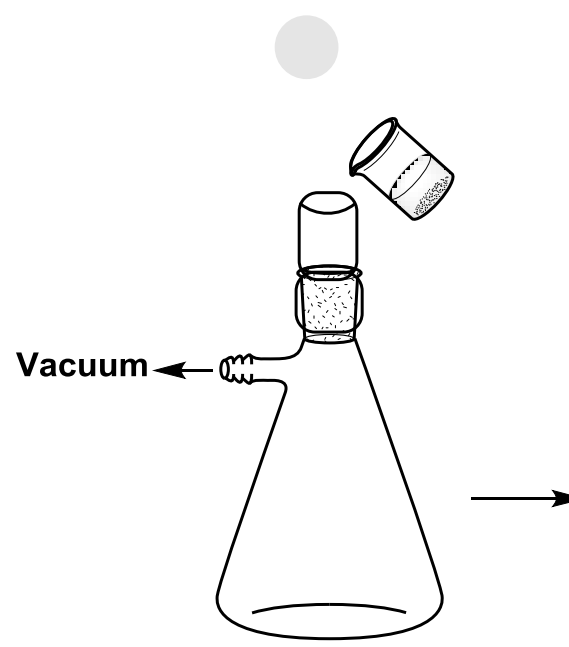

A: SWCNT loading

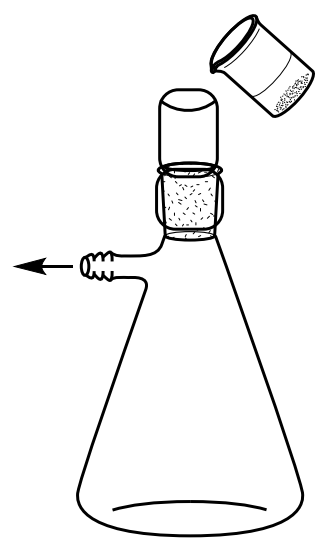

B: Washing

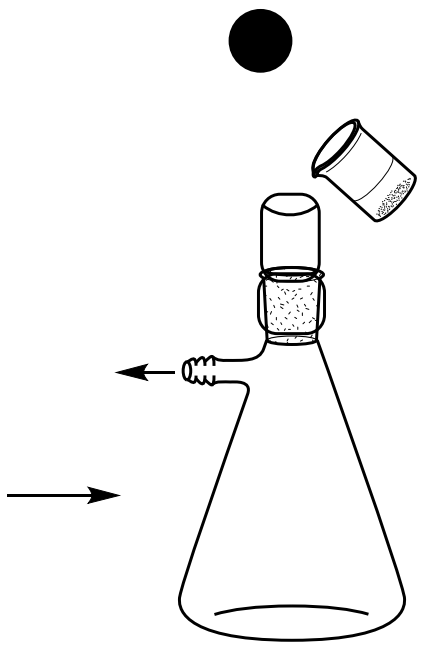

C: PtNP loading

Figure 31. Schematic representation of the preparation of paper-based PtNP/SWCNT hybrid thin film by vacuum filtration technique. (A) The SDS-dispersed aqueous solution of P-SWCNT was loaded on the surface of MCE membrane to form paper-based, porous P-SWCNT thin film. (B) The SDS molecules were washed away using an excess of water. (C) The citrate-capped PtNPs were loaded atop of P-SWCNT thin film to form paper-based PtNP/SWCNT hybrid thin film.

Figure 31 represents the schematic of fabrication of paper-based PtNP/SWCNT hybrid film by vacuum filtration method. During the vacuum filtration, we physically 
trapped citrate-capped PtNPs atop of the porous paper-based SWCNT thin film by using downward convective force. Pre-synthesized PtNPs ( $29 \mathrm{~nm})$ were deposited on the top of paper-based SWCNT film $\left(11.36 \mathrm{~cm}^{2}\right)$. Citrate-capped PtNPs (diameter $\sim 29 \mathrm{~nm}$ ) were chosen to demonstrate the proof-of-concept. The volume of PtNPs was chosen in such a way that the amount of platinum match with the selected electrochemically deposited sample $\left(73.6 \mu \mathrm{g} / \mathrm{cm}^{2}\right)$ that was confirmed by ICP/MS experiments.

\subsubsection{Characterization of synthesized PtNPs}

\subsection{Preparation and characterization of synthesized PtNPs}

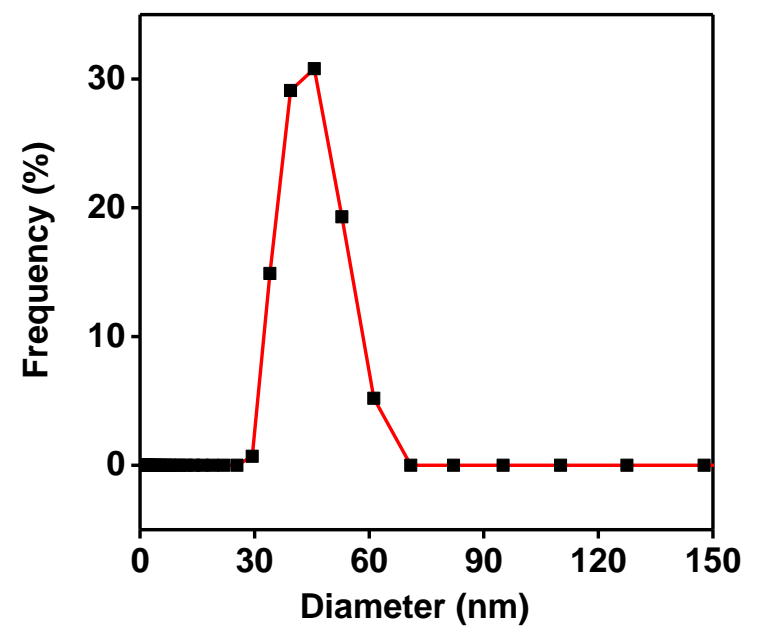

Figure 32. Characterization of PtNPs by dynamic light scattering (DLS) experiments. The synthesized PtNPs have the hydrodynamic size of $35 \pm 5 \mathrm{~nm}$ with a polydispersion index (PDI) of 0.2 .

PtNPs (diameter $~ 29 \mathrm{~nm}$ ) were prepared by reported seed-mediated method using chloroplatinic acid hexahydrate as a precursor salt. ${ }^{232}$ The PtNP seed (diameter $\sim 5 \mathrm{~nm}$ ) was prepared using a mixture of sodium citrate and citric acid as a stabilizer and sodium borohydrate as a reducing agent. Prepared PtNP seeds were further grown in the presence of precursor salt using ascorbic acid as a weak reducing agent. Figure 32 shows the hydrodynamic size and polydispersion index of the synthesized PtNPs. 


\subsection{The concentration of PtNP measured by ICP-MS}

Figure 33 represents a standard calibration curve of different concentration of platinum in the range of 0 to $100 \mathrm{ppm}$. The calibration curve was obtained with linear regression equation of $y=0.0029 x+0.0011\left(r^{2}=0.99992\right)$. By relating the observed counts (ratio) to the calibration curve, the concentration of PtNPs was determined to be $0.8552 \mathrm{mg} / \mathrm{ml}$.

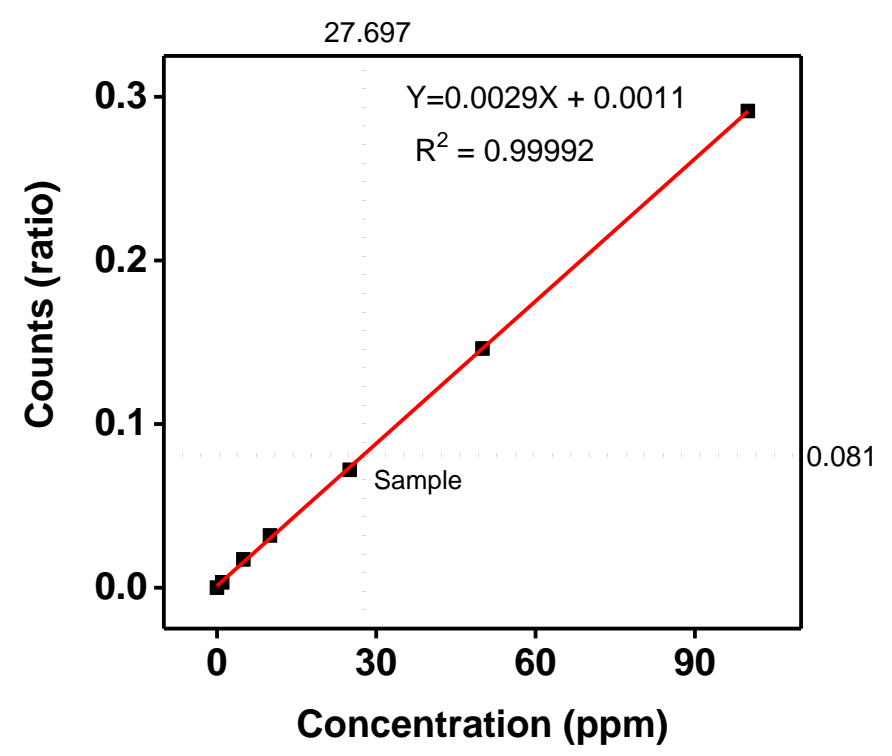

Figure 33. Calibration curve of standard ${ }^{95} \mathrm{Pt}$ for the determination of the amount of $\mathrm{Pt}$ of synthesized PtNPs. PtNPs were separated from the supernatant by three rounds of centrifugation and washing cycles.

PtNPs were separated from the supernatant by centrifugation $(7100 \mathrm{rpm}$ for 30 minutes) before ICPMS experiments. Re-dispersion and centrifugation (three times) with an equal volume of deionized water was used to clean PtNPs further. The total Pt concentration was determined in the supernatant, separated PtNPs and the whole reaction mixture without separation to validate the concentration of $\mathrm{Pt}$ in the sample. The slight difference $(\sim 2.3 \%)$ difference in concentration of Pt before and after washing showed negligible loss of PtNP during the washing process. 


\subsubsection{Characterization of paper-based PtNP/SWCNT hybrid film prepared by vacuum filtration}

\subsubsection{Pt concentration of the paper-based PtNP/SWCNT hybrid film}

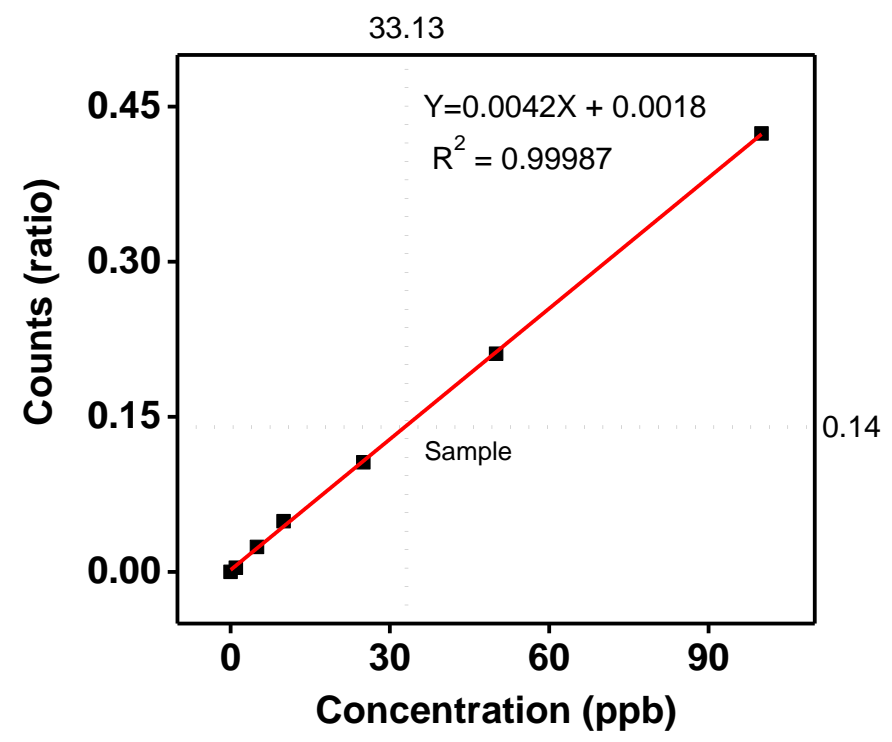

Figure 34. Calibration curve of of standard ${ }^{95} \mathrm{Pt}$ for determination of the amount of $\mathrm{Pt}$ on the surface of paper-based PtNP/SWCNT thin film prepared by vacuum filtration technique. The sample solution was prepared by dissolving Pt from the surface of electrode using aqua-regia solution.

To match the concentration of PtNPs of paper-based electrochemically deposited PtNP/SWCNT hybrid film, $13.1 \mathrm{ml}$ of prepared PtNPs was used to prepare the PtNP/SWCNT hybrid film on the paper by vacuum filtration method. In order to confirm the actual concentration of $\mathrm{Pt}$ on the hybrid film prepared using vacuum filtration technique, we performed ICP/MS experiments. Figure 34 represents the standard calibration curve of different concentration of ${ }^{95} \mathrm{Pt}$ that was used to calculate the concentration of $\mathrm{Pt}$ on the prepared film. The Pt concentration in the sample was determined by comparing the experimental value with the standard linear regression equation. 
Six different pieces of PtNP/SWCNT film (surface area $\sim 0.18 \mathrm{~cm}^{2}$ ) were selected and digested. Their concentration of Pt was determined by ICP/MS. We found that the average concentration of Pt from the film is same with that of PtNP solution employed for film fabrication (less than 1\% error), indicating that no PtNP was lost during the vacuum filtration. The average standard deviation of $\sim 6 \%$ indicated that even distribution of $\mathrm{Pt}$ within the entire film during the preparation. The concentration of $\mathrm{Pt}$ on the film was found to be almost similar to the sample prepared by electrodeposition method $(0.0736$ $\left.\mathrm{mgcm}^{-2}\right)$.

\subsubsection{Morphology of PtNPs on the surface of PtNP/SWCNT hybrid film}

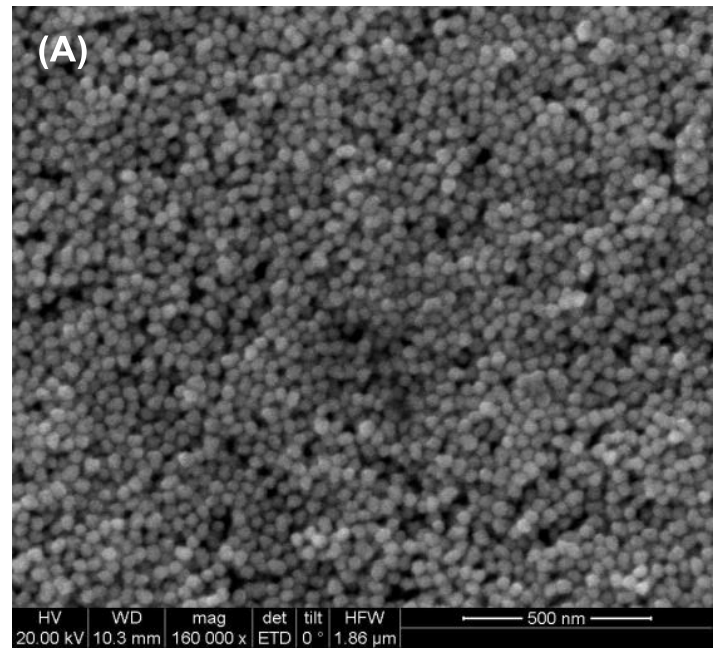

(B)

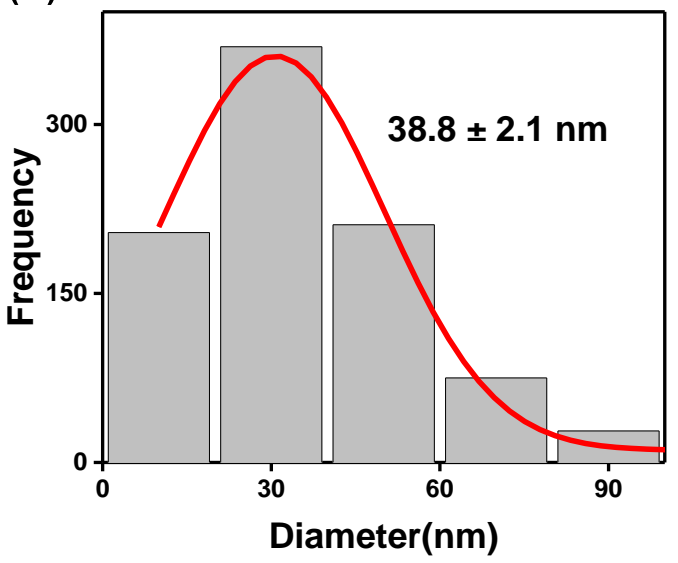

Figure 35. Morphology of of PtNP on the surface of PtNP/SWCNT hybrid film prepared by vacuum filtration. (A) FE-SEM images of paper-based PtNP/SWCNT hybrid film. (B) Size distribution of PtNPs analyzed using an imageJ software.

Figure 35A represents the morphology of platinum structures on paper-based PtNP/SWCNT hybrid film prepared by the vacuum filtration technique. Platinum is present as a continuous network of spherical platinum particles forming threedimensional nano-porous like structure atop of P-SWCNT film. The 3D porous 
continuous structure is different from the discrete platinum clusters observed with the electrochemically deposited PtNP/SWCNT hybrid film. We found that the average size of PtNPs was 38.8 \pm 2.1 . When compared the size distribution of electrochemically deposited PtNP/SWCNT hybrid film, it is clear that a relatively narrow size distribution of PtNPs was achieved using vacuum filtration technique.

\subsubsection{State of Platinum on the surface of paper-based PtNP/SWCNT hybrid film}

X-ray Photoelectron Spectroscopy (XPS) was used for speciation of platinum on paper-based PtNP/SWCNT hybrid film prepared by vacuum filtration technique.

A

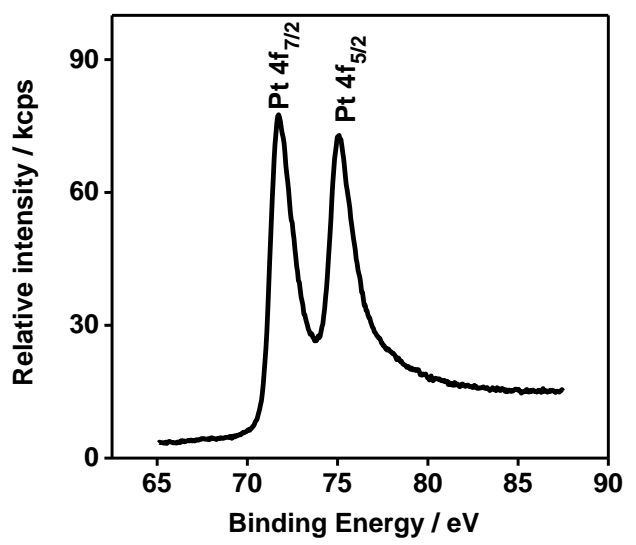

B

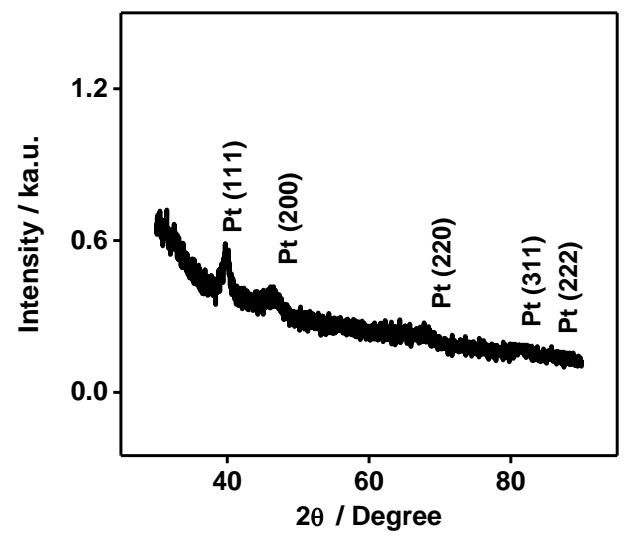

Figure 36. State of Pt on the surface of paper-based PtNP/SWCNT hybrid thin film prepared by vacuum filtration technique. (A) XPS spectra showing oxidation state of platinum and (B) XRD spectra showing the crystalline state of PtNP on the surface of SWCNT.

Figure 36A represents the XPS analysis of the PtNP/SWCNT composites showing platinum $4 f$ spectra, confirming the assembly of platinum on the surface of SWCNT film. The spectra are well-resolved with doublets of $\mathrm{Pt}_{4} \mathrm{f}_{7 / 2}$ and $\mathrm{Pt}_{4} \mathrm{f}_{5 / 2}$ showing the platinum is present in the form of $\mathrm{Pt}(0)^{95}$ with binding energies of $71.73 \mathrm{eV}$ and $75.18 \mathrm{eV}$, respectively. The higher intensity of $\mathrm{Pt}(0)$ in the sample prepared by vacuum filtration (2.84x) as compared to the sample prepared by electrodeposition indicates that higher 
surface area was achieved with vacuum filtration method.

Figure 36B represents the XRD pattern of $\mathrm{Pt}$ nanoclusters on the surface of PtNP/SWCNT hybrid film prepared by vacuum filtration technique. Peaks at $2 \theta=39.8^{\circ}$, $46.12^{\circ}, 67.52^{\circ}, 84.9^{\circ}$ and $87.04^{\circ}$ are assigned to crystalline planes of face-centered cubic of Pt (111), Pt (200), Pt (220), Pt (311) and Pt (222), respectively, indicating that PtNPs exist in crystalline form. ${ }^{119}$ We found that a very low intensity of crystalline planes in the sample prepared by vacuum filtration technique, as compared to the sample prepared by electrodeposition method. This is attributed to random but not a periodic distribution of Pt atoms (Pt nanoparticles) on the surface of P-SWCNT.

\subsubsection{Comparison of methanol oxidation of PtNP/SWCNT hybrid films}

A

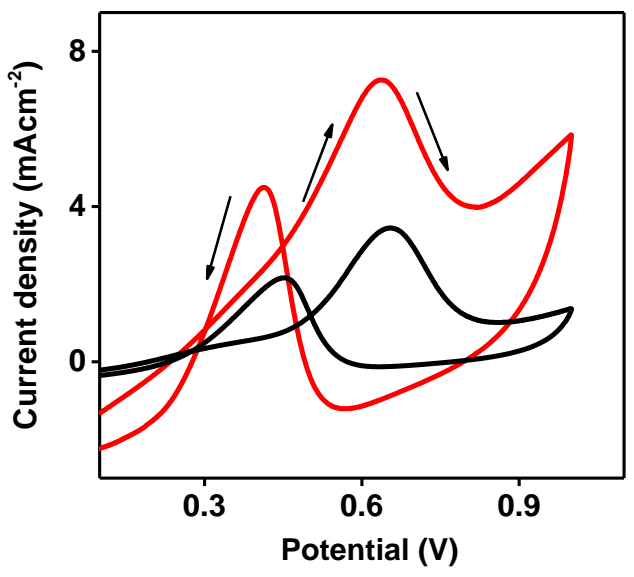

B

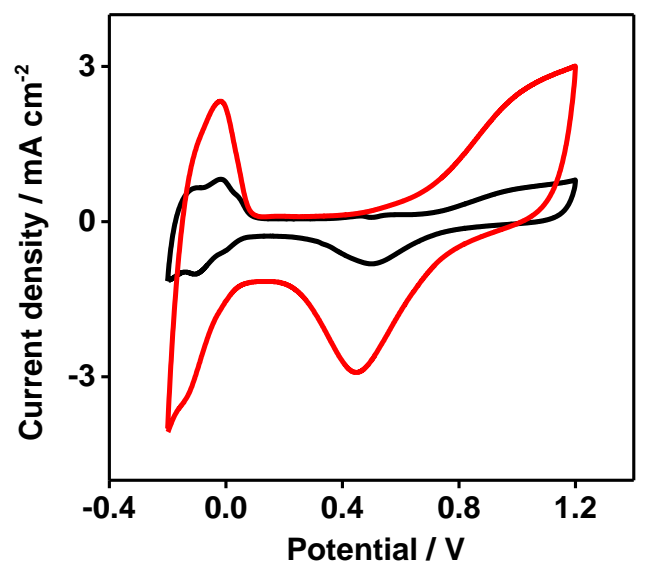

Figure 37. Comparison of electrocatalytic performance of PtNPs prepared by vacuum filtration and electrodeposition methods. (A) CV responses of PtNPs on paper-based SWCNT thin films prepared by vacuum filtration (red curve) or electrodeposition (black curve) in $0.1 \mathrm{M} \mathrm{H}_{2} \mathrm{SO}_{4}$ containing $0.1 \mathrm{M}$ methanol at $50 \mathrm{mV} / \mathrm{s}$. (B) Electroactive surface area of paper-based PtNP/SWCNT hybrid film prepared by vacuum filtration (red curve) or electrodeposition (black curve) in $0.1 \mathrm{M} \mathrm{H}_{2} \mathrm{SO}_{4}$ at $50 \mathrm{mV} / \mathrm{s}$.

We also evaluated the electrocatalytic activity of PtNP/SWCNT hybrid films for methanol oxidation in $0.1 \mathrm{M} \mathrm{H}_{2} \mathrm{SO}_{4}$ containing $0.1 \mathrm{M}$ methanol at the scan rate of 50 
$\mathrm{mV} \cdot \mathrm{s}^{-1}$ and compared the performance of the films prepared by vacuum filtration technique or electrodeposition method. Both films showed characteristic methanol oxidation curve with forward peak potential at $\sim 0.65 \mathrm{~V}$ and backward peak potential at $\sim 0.44 \mathrm{~V}$. With the same amount of Pt loaded on P-SWCNT film, the PtNP/SWCNT hybrid film fabricated with vacuum filtration showed significantly higher $(\sim 2.1 \times)$ forward peak current density as compared to the hybrid film prepared by electrodeposition method. We believe that the higher electrochemical activity is attributed to the porous, uniform and continuous platinum structure on the vacuum filtration fabricated sample as compared to discrete flower-like platinum clusters on the electrodeposition prepared sample.

The difference of methanol electrocatalytic response between vacuum filtration fabricated samples and electrodeposition prepared samples can be explained by the difference in electroactive surface area due to different size and orientation of platinum particles on P-SWCNT film. The roughness factor from hydrogen adsorption-desorption region was found to be 10.88 and 41.66 for vacuum filtration fabricated sample and electrodeposition prepared samples, respectively. The mass specific electroactive surface area of platinum was calculated as $14.1 \mathrm{~m}^{2} / \mathrm{g}$ (electrodeposition) and $42.3 \mathrm{~m}^{2} / \mathrm{g}$ (vacuum filtration), which is much higher than bulk $\mathrm{Pt}\left(2.8 \mathrm{~m}^{2} / \mathrm{g}\right){ }^{237}$ The electroactive surface area was $\sim 0.6 \times$ and $1.8 \times$ higher as compared to previously reported PtNP/SWCNT hybrid film ${ }^{237}$ prepared by vacuum filtration of pre-mixed PtNP and CNT solution.

Both samples showed much better $\mathrm{CO}$ tolerance as compared to commercially available E-TEK catalyst. ${ }^{113}$ The ratio of forward peak current to backward peak current was taken as indicator of tolerance of $\mathrm{CO}$ poisoning of an electrode. This ratio was found to be 1.65 for vacuum filtration fabricated sample and 1.60 for electrodeposition 
prepared sample which is much larger as compared to commercially available E-TEK catalyst $(0.74){ }^{113}$ This is attributed to the porous structure of P-SWCNT thin film where oxidative carbon species can be easily moved away from the surface of the electrode.

\subsubsection{Stability and reproducibility of PtNP/SWCNT hybrid film prepared vacuum filtration technique}

We evaluated the stability and reproducibility of the PtNP/SWCNT hybrid thin film prepared by vacuum filtration by measuring the anodic current for the oxidation of methanol. The oxidation peak current density for $0.1 \mathrm{M}$ methanol in $0.1 \mathrm{M} \mathrm{H}_{2} \mathrm{SO}_{4}$ solution at peak potential of $0.65 \mathrm{~V}$ was chosen for the measurement.
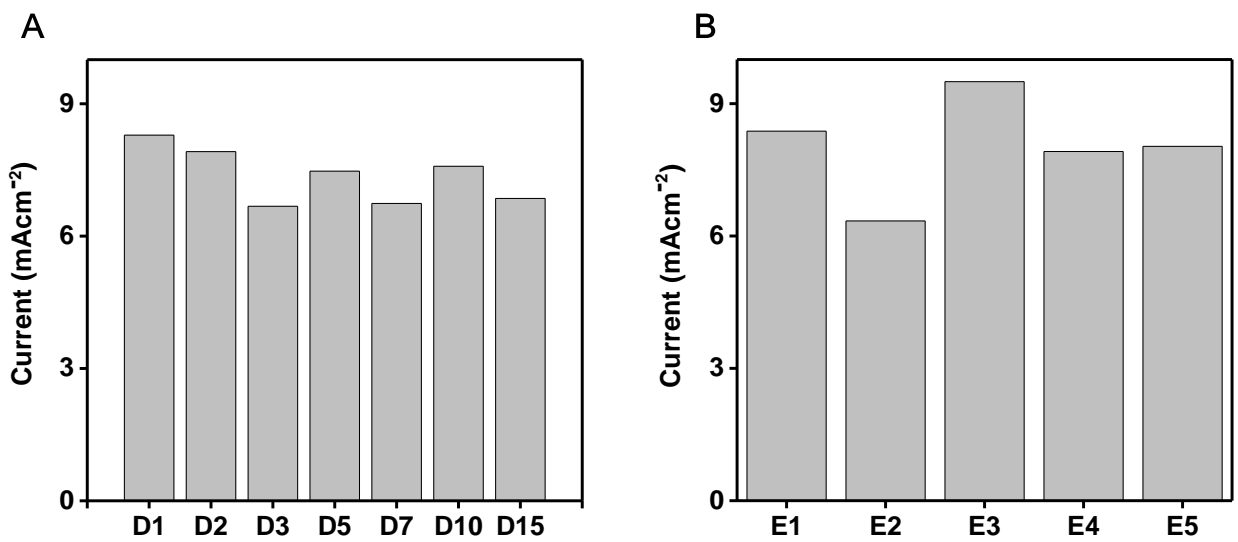

Figure 38. Stability and reproducibility of paper-based PtNP/SWCNT hybrid film prepared by vacuum filtration. (A) Stability and (B) reproducibility of different film electrodes in $0.1 \mathrm{M}$ methanol oxidation in $0.1 \mathrm{M} \mathrm{H}_{2} \mathrm{SO}_{4}$ solution.

Figure 38A represents the stability of the prepared films tested over 15 days. The average standard deviation of peak current is about $9 \%$, which indicated that the prepared film has good stability. The reproducibility of the film was evaluated by randomly choosing five different electrodes from the prepared film (Figure 38B). The calculation shows the reproducibility of the prepared film is within $14 \%$ of the average value. These values have shown that hybrid thin films also have good reproducibility. 


\subsection{Broader Impacts}

We have demonstrated the functionalization of paper-based SWCNT thin film by electrodepositing platinum nanoparticles. As compared to common solid surface electrodes, the PtNPs electro-deposited on the surface of paper-based SWCNT film generated higher electroactive surface area and higher electrocatalytic performance towards methanol oxidation. We further demonstrated the use of paper-based SWCNT thin film as a conducting support by depositing PtNPs on its surface by routine vacuum filtration technique. The newly formed PtNP/SWCNT hybrid film showed a 3D, continuous and porous platinum structure with a relatively narrow size distribution of PtNPs, resulting in the higher electroactive surface area and higher electrocatalytic response towards methanol as compared to the electrochemically deposited sample. This fabrication process provides excellent alternatives for simple, fast and cheap fabrication of PtNP/SWCNT hybrid film. The flexibility of vacuum filtration could easily be extended to a different size, volume, and shape of PtNPs and its alloys for more efficient PtNP/SWCNT composite materials. We envision that flexibility of paper and broad functionality of platinum can be used to incorporate the paper-based Pt-SWCNT composite material in the development of portable sensors, fuel cells, and solar cells. 
Chapter 5. A colorimetric assay for detecting DNA and exonuclease III based on DNA-modified gold nanoparticles

\subsection{Introduction}

DNA modifying enzymes such as polymerases, endonucleases and exonucleases are an integral part of genomic stability due to their prominent role in many biological processes such as recombination, repair, replication, cloning, genotyping and mapping of nucleic acids. ${ }^{179,238}$ In particular, 3' to 5' exonucleases such as E. coli exonuclease III (Exo III) and Mre 11 protein are involved in DNA proofreading and repair through their 3' to 5' exonuclease activity. ${ }^{187,239,240}$ Similarly, Class II Apurinic/apyrimidinic (AP) endonucleases such as exonuclease III and APE1 are involved in recognition and processing of AP sites in Base excision repair through their AP endonuclease activity. ${ }^{241-}$ 243

Based on multi-functions of class II AP endonucleases within cells, numerous studies have reported that elevated or altered expression of these endonucleases in breast, ${ }^{244}$ cervical $^{245}$ and non-small cell lung cancers. ${ }^{246}$ Therefore, the expression of these enzymes can be used as a potential biomarker for the prediction of carcinogenesis risk, ${ }^{247}$ or as a druggable target, ${ }^{185}$ or design, and development of inhibitors ${ }^{186,248,249}$ or in mechanistic study of different cancers. ${ }^{250}$ Similarly, $3^{\prime}-5^{\prime}$ exonucleases are involved in different biological process such as DNA proofreading. Their overexpression in human cells is believed to be related to increased longevity owing to stabilization of the mutation rates in a cell, whereas organisms that lack $3^{\prime}-5^{\prime}$ exonucleases are found to be more susceptible to cancer, especially under stress conditions. ${ }^{187,240}$ Therefore, these enzymes are good target for drug development and radio and/or chemotherapeutic treatments. 
Exonuclease III is major prokaryotic DNA modifying enzyme in Escherichia coli. ${ }^{175} \mathrm{It}$ has AP endonuclease activity and cleaves the phosphodiester bond in duplex DNA immediately $5^{\prime}$ to the abasic sites to produce $3^{\prime} \mathrm{OH}$ group. ${ }^{176-178}$ It has the structural and functional similarity with other class II AP endonucleases such as APE1 of human cells and Apn1 of yeast and often used as a model enzyme for the mechanistic study. ${ }^{179,180}$ Similarly, Exonuclease III also has 3' to 5' exonuclease and removes the mononucleotides from 3' end or nicks of double strand DNA. ${ }^{178,181,182}$ Beside AP endonuclease and 3' to 5' exonuclease activity, Exonuclease III has RNase $\mathrm{H}$ and 3' phosphatase activity.

Exonuclease III was chosen as a target to demonstrate the proof of concept of our assay due to its commercial availability. Various methods have been developed for detection of the activity of Exonuclease III. The most common method is gel electrophoretic-based radiographic assay. ${ }^{192,193}$ The radioisotope $\left({ }^{3} \mathrm{H}\right.$ or $\left.{ }^{32} \mathrm{P}\right)$ labeled DNA substrate was reacted with exonuclease III and digested products are then separated using gel electrophoresis. The concentration and the length of the digested products were determined by comparing the radioactivity of control sample with the products. These assays require stringent safety procedure to control radioactive exposure. Furthermore, these gel-based assays are laborious, requiring lengthy time for the preparation and analysis of gel. As an alternative, fluorescence assays have been developed for quick enzyme analysis in high throughput screening fashion. These solution methods use fluorescence label or label-free DNA substrate for the enzymatic reaction. For example, Wu et al. ${ }^{194}$ used fluorescence labeled molecular beacon as a DNA substrate for the detection of exonuclease activity. The hairpin structure of molecular beacon was modified with a fluorophore (FAM) at its 5 ' end and the quencher (BHQ1) at its 3' end. The fluorescence was quenched in the absence of enzyme due to 
effective forster resonance transfer mechanism (FRET). However, in the presence of the enzyme, it releases the quencher from the molecule beacon due to its $3^{\prime}$ to $5^{\prime}$ activity, which increases the fluorescence of the solution. The change in fluorescence was used as a measure of activity of Exonuclease III. Alternatively, Su et al. ${ }^{195}$ used stacked guanines instead of BHQ1 at the 3' end as a quencher to reduce the cost of the assay. To further simplify the fluorescence assays, label-free fluorescence assays have been developed based on the formation of the G-quadruplex structure upon exonuclease III reaction from linear DNA sequences. The enzyme activity was then monitored by measuring the fluorescence of G-quadruplex-specific dyes such as crystal violet ${ }^{196}$ or $\mathrm{Tb}_{2} \mathrm{O}_{3} \cdot{ }^{197}$

Gold nanoparticles (AuNPs) as a colorimetric probe provides an excellent alternative to traditional gel or fluorescence methods due to their intense optical properties and chemical stability. ${ }^{251-253}$ In particular, the color change can be observed by the naked eye without the need of an instrument. Therefore, DNA-AuNPs have been successfully used as colorimetric probe for the detection of nucleic acids, ${ }^{254}$ proteins, ${ }^{255}$ metal ions, ${ }^{160}$ small molecules ${ }^{162,256}$ and enzymes. ${ }^{155,156,161}$

Here, we have reported a novel colorimetric assay for detecting the activity of Exonuclease III based on the non-cross linking mechanism of AuNP aggregation induced by enzymatic removal of DNA substrate from the DNA-modified AuNPs. This method enables naked-eye colorimetric monitoring of AP endonuclease activity and can be used for high throughput screening of AP endonucleases inhibitors. Compared to the previous assays, we designed the DNA substrate for both Exonuclease III 3' to 5' exonuclease and its AP endonuclease activity. 


\subsection{Experimental approach}

Materials and Reagents. All the chemicals including Trizma, Tris-acetate, potassium acetate, sodium chloride, magnesium chloride, calcium chloride, gold (III) chloride tri-hydrate, tri-sodium citrate, dithiothreitol (DTT) were purchased from SigmaAldrich. All DNA sequences were purchased from Integrated DNA Technology (IDT). Following DNA sequences were used in this work.

\begin{tabular}{|c|c|}
\hline DNA probe ID & Sequences \\
\hline OX_Probe_FAM & $\begin{array}{l}\text { 5'-SH TTT TTT ACC ACA TCA TCC } \text { ATA TAA CTG AAA } \\
\text { GCC AAACAG- FAM-3' }\end{array}$ \\
\hline 1X_probe_FAM & $\begin{array}{l}\text { 5'-SH TTT TTT ACC ACA TCA TCCX TAT AAC TGA AAG } \\
\text { CCA AAC AG- FAM-3' }\end{array}$ \\
\hline 1X_probe & $\begin{array}{l}\text { 5'-SH TTT TTT ACC ACA TCA TCCX TAT AAC TGA AAG } \\
\text { CCA AAC AG-3' }\end{array}$ \\
\hline $\begin{array}{l}\text { Complementary } \\
\text { DNA }\end{array}$ & 5'-CTG TTT GGC TTT CAG TTA TAT GGA TGA TGT GGT-3' \\
\hline
\end{tabular}

Synthesis of gold nanoparticles (AuNPs). Gold nanoparticles of $\sim 13 \mathrm{~nm}$ were prepared by previously reported Fren's citrate method. ${ }^{257}$ Briefly, all glasswares were incubated in aqua regia overnight and washed with deionized water before use. $96 \mathrm{ml}$ of water was boiled in RB flask with vigorous stirring. $2.5 \mathrm{ml}$ of $100 \mathrm{mM}$ freshly prepared gold (III) chloride trihydrate solution was then added. Immediately, after addition of gold salt, $2 \mathrm{ml}$ of $34 \mathrm{mM}$ sodium citrate solution was added rapidly which acts as both reducing and stabilizing agent. The formation of gold nanoparticle was indicated by the appearance of red color due to the reduction of $\mathrm{Au}^{+3}$ to $\mathrm{Au}^{0}$. The solution was kept 
boiling for another 30 minutes and cooled down to room temperature under stirring. The solution was filtered using $0.22 \mu \mathrm{m}$ syringe filter and used immediately for DNA modification experiments.

UV-visible experiments. Prepared gold nanoparticle solution was $5 \mathrm{X}$ diluted and absorbance was measured by using Cary 100 Bio UV-Vis spectrophotometer. The absorbance of DI water was taken as a background and subtracted from the absorbance of gold nanoparticle solution.

Dynamic light scattering experiments. The hydrodynamic radius of citrate-capped gold nanoparticles was determined using Malvern DLS Zeta sizer Nano. $1.2 \mathrm{ml}$ of asprepared AuNPs was used to determine the hydrodynamic radius.

DNA modification of gold nanoparticles. Different DNA probes were modified on the surface of AuNPs by reported method ${ }^{151}$ with slight modification. Briefly, disulfide bond of DNA probes was cleaved by reacting it with freshly prepared TCEP solution (3 mg per $100 \mu \mathrm{l})$. The TCEP and DNA were mixed in the ratio of 75:1 and reacted for $2 \mathrm{hrs}$ in the dark at room temperature. The mixture was used immediately for modification without purification. The mixture of TCEP-DNA was added into $3 \mathrm{ml}$ of AuNPs slowly with stirring so that the final concentration of DNA was $3 \mu \mathrm{M}$. The reaction was incubated for $12 \mathrm{hrs}$ at room temperature before salt addition. The concentration of $\mathrm{NaCl}$ was then increased to $0.05 \mathrm{M}$ using $2 \mathrm{M} \mathrm{NaCl}$ solution dissolved in $100 \mathrm{mM}$ PBS solution ( $\mathrm{pH} 7.0)$ containing $0.01 \%$ SDS. The solution was then sonicated briefly for $\sim 10$ s and allowed to incubate for extra 20 mins. The process was repeated for an increment of $0.05 \mathrm{M} \mathrm{NaCl}$ once and for every $0.1 \mathrm{M} \mathrm{NaCl}$ after that, until the final concentration of $\mathrm{NaCl}$ reached $1.0 \mathrm{M}$. Once the $\mathrm{NaCl}$ concentration reached $1 \mathrm{M}$, the solution was kept for another 12 hrs before the separation. After modification, the DNA-modified AuNPs were separated from excess of DNA by using centrifuge column (100 KD cut-off, Millipore, USA). The 
separation was carried out by centrifugation at 1000 rpm for 20 minutes. The DNAmodified AuNPs were further washed 6 times with $1 \mathrm{X}$ Tris buffer $(\mathrm{pH} 7.4)$ to remove excess of salt and DNA using same centrifugation process. The DNA-modified AuNPs were dissolved in $300 \mu \mathrm{l}$ Tris buffer $(\mathrm{pH} 7.4)$ and stored at $4{ }^{\circ} \mathrm{C}$ before use.

Determination of Surface coverage of FAM-labeled probe. The surface coverage of DNA in gold nanoparticle was determined from previously reported method. ${ }^{151}$ To determine the surface coverage, the concentration of nanoparticle and DNA probes on the surface was determined separately. The concentration of AuNP was determined directly from UV-visible experiments whereas the concentration of DNA was determined after displacing it from the surface using DTT. To displace DNA from the surface, $2 \mu \mathrm{l}$ of DNA-modified gold nanoparticle was mixed with $48 \mu \mathrm{l}$ of $1 \mathrm{X}$ Tris-acetate. The diluted solution was mixed with $50 \mu$ of $1 \mathrm{M}$ of DTT in $1 \mathrm{X}$ Tris -acetate buffer and reacted overnight to completely displace DNA probes from the surface of AuNP. The Gold precipitate was removed by centrifugation (21000 rpm for 20 minutes). Standard DNA samples (0 to $200 \mathrm{nM}$ ) were also prepared by treating with the same amount of DTT in $1 \mathrm{X}$ Tris-acetate buffer $\mathrm{pH}$ 7.9. $60 \mu \mathrm{l}$ of supernatant was placed into 96-plate reader and fluorescence was compared with the standard curve of FAM-labeled DNA probe solution. During fluorescence measurement, fluorophore was excited at $495 \mathrm{~nm}$ and emission was recorded at $520 \mathrm{~nm}$.

Determination of Surface coverage of label-free probe. The surface coverage of label-free DNA probe was determined by the similar method except DNA concentration was determined by using oligreen assay. $20 \mu \mathrm{l}$ of the supernatant solution was diluted to $100 \mu \mathrm{l}$ solution using $1 \mathrm{X}$ TE buffer. To this $100 \mu \mathrm{l} 1 \mathrm{X}$ oligreen dye was added. The mixture was incubated in the dark for 5 minutes before measuring the fluorescence. 60 $\mu \mathrm{l}$ of the mixture was taken out and placed in 96 plate reader for fluorescence 
measurements. The standard solutions were also prepared similarly in the range of 0 to $200 \mathrm{nM}$. The fluorophore was excited at $500 \mathrm{~nm}$ and emission was recorded at $525 \mathrm{~nm}$.

Kinetics of DTT reaction on DNA-modified gold nanoparticle probes. Prepared DNA-modified gold nanoparticles were diluted 25X using 1X Trizma buffer. $25 \mu \mathrm{l}$ of the diluted solution was mixed with $10 \mu \mathrm{l}$ of different concentrations of DTT in 1X Trizma buffer so that final concentration of the DTT becomes 0 to $50 \mu \mathrm{M}$. The kinetics of displacement or change in the configuration of FAM-labeled DNA was monitored by measuring the change in fluorescence of the FAM labeled DNA for 30 minutes. The FAM was excited at $495 \mathrm{~nm}$ and emission was collected at $520 \mathrm{~nm}$.

DDT treatment of DNA-modified AuNPs. $50 \mu \mathrm{l}$ of (25X diluted in $1 \mathrm{X}$ Tris-acetate buffer $\mathrm{pH}$ 7.9) DNA-AuNPs was mixed with $20 \mu \mathrm{l}$ of the different concentrations of DTT (diluted in $1 \mathrm{X}$ Tris-acetate buffer $\mathrm{pH}$ 7.9) and reacted for 30 minutes. The excess amount of DTT was removed by three cycles of washing and centrifugation steps (each at 21000 rpm for 10 minutes), and the supernatant was replaced with the equivalent amount of $1 \mathrm{X}$ Tris-acetate buffer.

Stability of DTT treated samples Stability of DTT-treated DNA modified AuNPs was tested by dispersing it in exonuclease III buffer containing $10 \mathrm{mM}$ tris acetate, 50 $\mathrm{mM}$ potassium acetate, $25 \mathrm{mM} \mathrm{NaCl}, 3 \mathrm{mM} \mathrm{MgCl}_{2}$, and $20 \mathrm{mM} \mathrm{CaCl} 2 \mathrm{pH} 7.9$ before further experiments. The stability towards the aggregation of particles was tested by measuring the UV-visible spectra and by observing the visual change in color.

Exonuclease activity on DTT treated samples The DTT treated samples were finally dispersed in exonuclease III buffer (10 mM tris acetate $\mathrm{pH} \mathrm{7.9,50} \mathrm{mM} \mathrm{potassium}$ acetate, $25 \mathrm{mM} \mathrm{NaCl}, 3 \mathrm{mM} \mathrm{MgCl}_{2}$, and $20 \mathrm{mM} \mathrm{CaCl}_{2}$ ) to which $100 \mathrm{nM}$ of target DNA was added and reacted with 0.2 units $\mu \mathrm{l}^{-1}$ of exonuclease III. The exonuclease activity was determined by measuring the extent of aggregation of gold nanoparticles as 
indicated by the ratio of absorbance at $650 \mathrm{~nm}$ to the absorbance at $520 \mathrm{~nm}$ in UV-visible experiments. To compare exonuclease activity in different probes, optimum DTT concentration for each probe was used.

Optimization of salt conditions for exonuclease assay Samples were pre-treated with DTT as described above. The DTT treated samples were finally dispersed in $1 \mathrm{X}$ tris acetate buffer $\mathrm{pH} 7.9$ containing different concentrations of $\mathrm{NaCl}$ or $\mathrm{MgCl}_{2}$. To this, 50 $\mathrm{nM}$ of target DNA and 0.2 units/ $\mu$ l was added. The effect was monitored by monitoring the extent of aggregation of AuNPs as indicated by the ratio of A650/A520 in UV-visible experiments.

Colorimetric exonuclease assay The DTT treated samples were finally dispersed in optimized buffer i.e. 1x Tris-acetate buffer $\mathrm{pH} 7.9$ containing $6 \mathrm{Mm}$ of $\mathrm{MgCl}_{2}$. To this, $50 \mathrm{nM}$ of target DNA was added before adding different amounts of exonuclease III. The exonuclease III was detected either by visual assessment of change in color from red to blue or by the ratio of A650/A520 in UV-visible experiments.

\subsection{Results and Discussion}

\subsubsection{DNA probe design}

The DNA probe was designed to accommodate both $3^{\prime}$ to $5^{\prime}$ exonuclease and AP endonuclease activities of exonuclease III. This is in contrast to traditional exonuclease III assays based on its 3' to 5' exonuclease activity. By designing new probe, we hypothesized that the assay would be rapid due to the employment of both 3 ' to 5 ' exonuclease activity of Exonuclease III and its AP endonuclease activity. Figure 39A represents the traditional probe design for the exonuclease activity without an abasic site (0X). The probe hybridized with the target to form a DNA duplex. In the presence of exonuclease III, mononucleotides were released from 3' end due to its 3 ' to 5 ' exonuclease activity. The newly designed probe incorporated a 3'-end abasic site (1X) 
at position 21. When hybridized with the target, it formed a DNA duplex that was the substrate for both AP endonuclease activity as well as 3' to 5' endonuclease activity as shown in figure 39B. The AP endonuclease activity of Exonuclease III hydrolyzes the phosphodiester bond 3' to the AP site forming 3' $\mathrm{OH}$ group. The formed nick with 3'OH group then became a new substrate for 3' to 5' exonuclease activity. Therefore, in contrast to the traditional assays, exonuclease acted on multiple sites of a single DNA duplex with dual activities, which greatly enhanced the cleavage rate of DNA substrate.

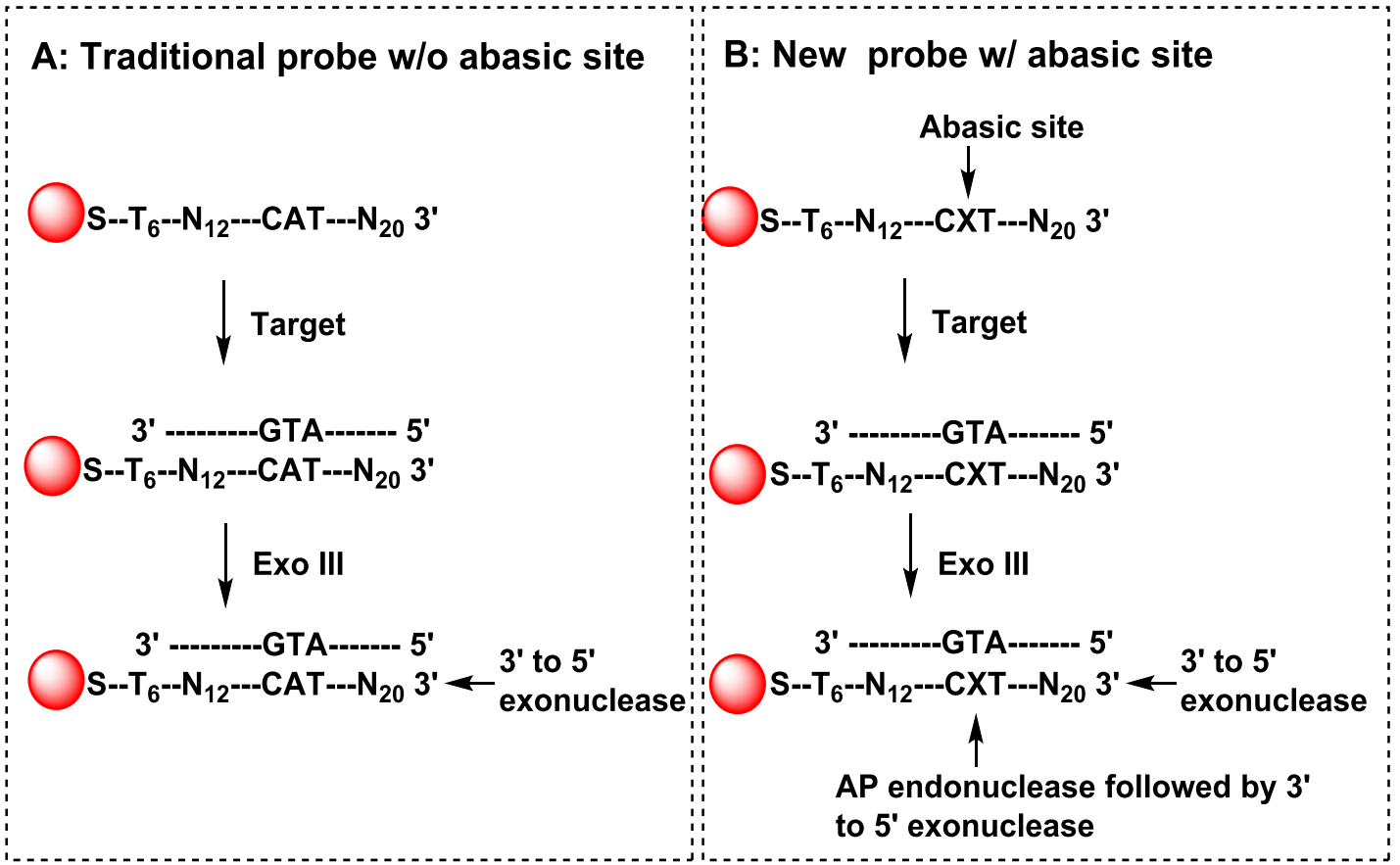

Figure 39. The design of DNA probes for determining the activity of Exonuclease III. (A) Traditional probe without an abasic site is the substrate for its 3' to 5' exonuclease activity. (B) New probe substrate incorporated with AP site, is the substrate for both 3' to 5 ' exonuclease activity and AP endonuclease activity.

Furthermore, the probe DNA (1X probe) with 38 nucleotides and an abasic site was designed as follows: 5'- thiol-poly $\left(\mathrm{T}_{6}\right)-(\mathrm{N})_{12}-\mathrm{X}-(\mathrm{N})_{20}-\mathrm{FAM}-3$ '. The 5' end was modified with the thiol group to attach it on the surface of AuNP whereas 3' end is either 
label-free or fluorescently labeled with the FAM. Poly $\left(T_{6}\right)$ was added to increase the flexibility of probe on the surface of AuNPs.

\subsubsection{DNA modification on gold nanoparticles}

\subsubsection{Characterization of gold nanoparticles}
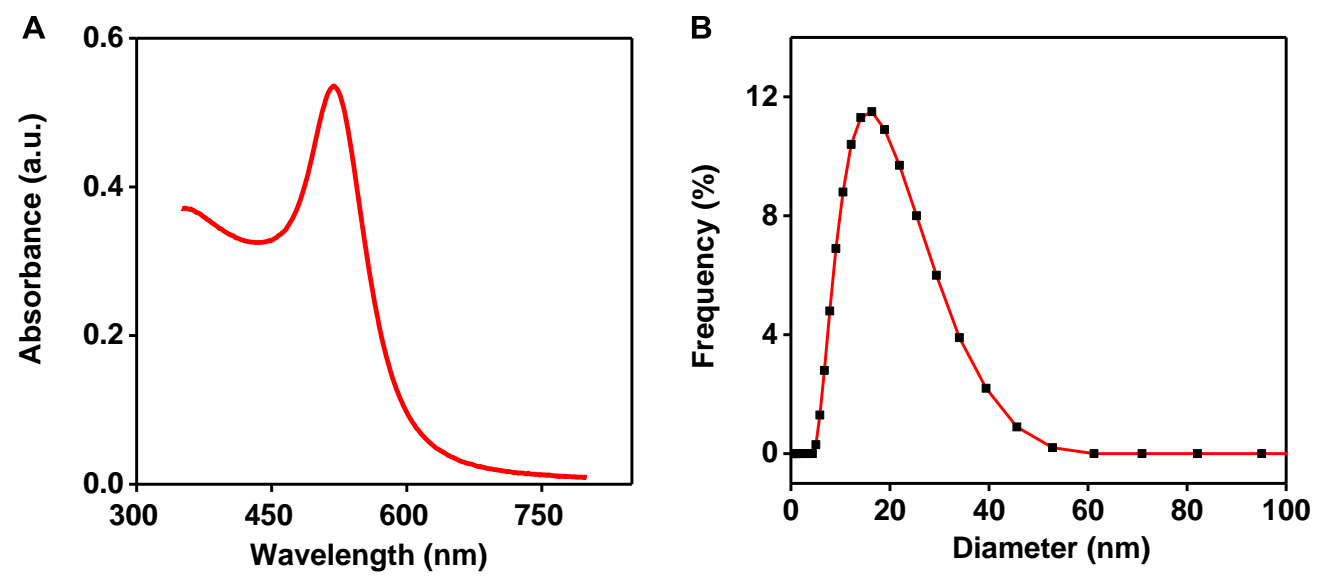

Figure 40. Characterization of gold nanoparticles. (A) UV-visible spectra of the citratecapped gold nanoparticle of $\sim 13 \mathrm{~nm}$ size showing a characteristic peak at $519 \mathrm{~nm}$ and (B) Dynamic light scattering spectra of prepared gold nanoparticle showing the hydrodynamic radius of $\sim 18 \mathrm{~nm}$ with PDI of 0.10 .

The citrate-coated gold nanoparticles of $\sim 13 \mathrm{~nm}$ were prepared by chemical reduction method as described in the procedure. Figure 40A represents the UV-visible spectra of prepared AuNPs showing the characteristic peak at $519 \mathrm{~nm}$. The concentration of AuNPs was determined from UV-Visible spectroscopy using Beer's law. ${ }^{258}$ The molar concentration was found to be $9.83 \mathrm{nM}$ when the molar extinction coefficient of $2.7 \times 10^{8} \mathrm{~cm}^{-1} \mathrm{M}^{-1}$ was used. Figure $40 \mathrm{~B}$ shown the hydrodynamic radius is $18 \mathrm{~nm}$ with a small poly dispersion index (PDI) of 0.10 .

\subsubsection{Modification of probe DNA on the surface of AuNPs}

5' thiol modified $1 \mathrm{X}$ probe DNA was attached to the surface of the AuNPs using standard gold-thiol chemistry whereas its 3 ' end was used for exonuclease III reaction. 
The modification was carried out using previously reported method with slight modification (see experimental section). The 3' end of probe DNA was labeled with FAM (1X_FAM) to study the effect of DTT on probe DNA modified AuNPs. The probe DNA without incorporation of an abasic site (0x probe) was also modified with AuNPs to compare exonuclease assays based on only exonuclease activity or dual activities.

\subsubsection{DNA probe density on AuNPs}

DNA density was calculated by dividing molar concentration of immobilized DNA by molar concentration of the AuNP. The molar concentration of AuNP was determined directly from UV-visible experiments. ${ }^{258}$ The concentration of FAM-labeled DNA immobilized on the surface of AuNP could not measure directly due to the quenching effect of AuNPs on labeled FAM fluorophore molecules. ${ }^{259}$ Therefore, FAM-labeled DNA probes were displaced by DTT ${ }^{151}$ and separated from AuNPs for the measurement.

Figure 41 represents the schematic of DTT displacement assay for the determination of DNA concentration on the surface of AuNP. The thiol-modified DNA was displaced from the surface of AuNP using ligand exchange reaction and separated from AuNPs using centrifugation. The fluorescence of released DNA was then measured, and the concentration of DNA was calculated with the established calibration curve.

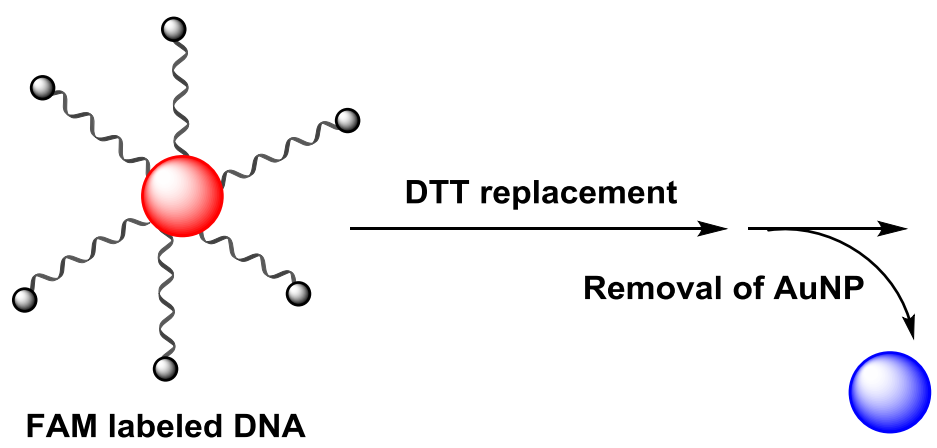

(Fluorescence off)

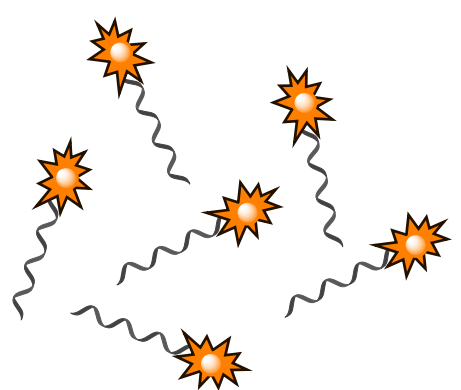

(Fluorescence on)

Figure 41. Schematic representation of DTT displacement fluorescence assay for the determination of the concentration of fluorescence labeled DNA on the AuNPs. 
Figure 42 represents the calibration curve of the standard solution of $1 \mathrm{X}$ probe in the range of 0 to $250 \mathrm{nM}$ with linear regression equation of $=178.07 C(n M)-541.87(r=$ 0.9993). The DNA probe concentration on the surface of AuNP was calculated by using the standard calibration curve. The surface coverage was found to be $106 \pm 5$ strands per AuNP (33.17 $\pm 1.5 \mathrm{pmole} / \mathrm{cm}^{2}$, by considering the 13-nm sized spherical AuNPs). Using similar method, the surface coverage for controlled OX_FAM probe was found to be $95 \pm 4$ strands per AuNP $\left(29.7 \pm 1.25 \mathrm{pmole} / \mathrm{cm}^{2}\right)$. The 0X_FAM and 1X FAM probe modified AuNPs were also used to compare exonuclease III activity due to their comparable surface density.

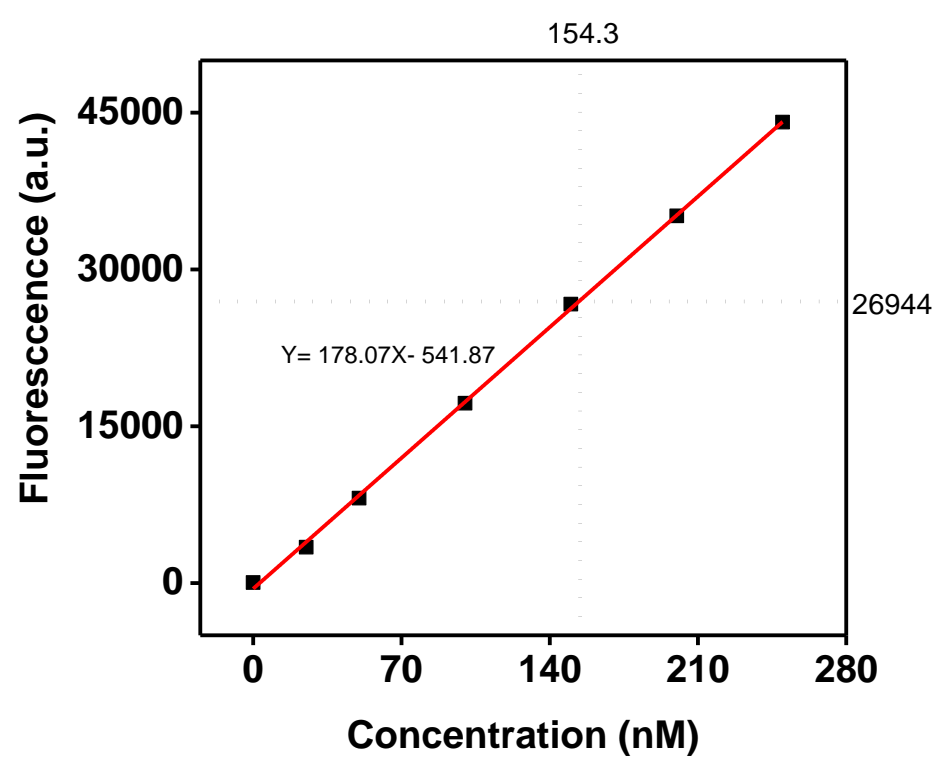

Figure 42. The calibration curve of the standard solution of 1X_FAM probe in the range of 0 to $200 \mathrm{nM}$ for the calculation of DNA probe density on the surface of AuNPs. Both standard solutions and DNA-modified AuNPs were reacted for $12 \mathrm{hrs}$ with an equal volume of $1 \mathrm{M}$ DTT before measuring the fluorescence. 


\subsubsection{Principle of colorimetric assay based on exonuclease III assisted target recycling for determining the activity of Exonuclease III}

The assay is based on non-cross-linking aggregation of DNA-modified gold nanoparticles induced by removal of DNA probes from the AuNPs. Exonuclease assisted target recycling strategy has been used to amplify the signal for sensitive target

detection. ${ }^{189,260} \mathrm{~A}$ low target concentration can be used to monitor the enzyme activity. This is significant in non-cross-linking AuNP-based colorimetric assays, as an aggregation of AuNPs induced by the hybridization of DNA at the concentration of 500 $\mathrm{nM}$ has been reported. ${ }^{261}$

Figure 43 represents the schematic of a colorimetric assay for determining the activity of Exonuclease III based on the enzymatic cleavage of DNA substrate. The DNA probe immobilized on the surface of the gold nanoparticle (Probe-AuNP) acts as both colloidal stabilizer of AuNP as well as a recognition element for its complementary target DNA. The AuNP-attached probe hybridized with its complementary target to form exonuclease specific DNA duplex substrate. In the absence of exonuclease III, the AuNP-attached DNA remained intact; therefore, it prevented the aggregation of AuNPs and was red due to electrostatic repulsion. In the presence of exonuclease III, duplex DNA on the surface of the AuNP was recognized and mononucleotides was released from 3' hydroxyl end of duplex DNA. Meanwhile, its AP endonuclease activity recognized the AP site of duplex DNA and underwent AP endonuclease activity to form a new 3' $\mathrm{OH}$ nick. The newly formed 3' $\mathrm{OH}$ nick also became the substrate for its exonuclease activity and started to release mononucleotides from the probe DNA and ultimately released intact target DNA into the solution. The released target hybridized with another probe on the surface of the gold nanoparticle and formed a new duplex to restart the cycle. This 
cycle repeats until almost all the probes were removed from the AuNPs. The sheared AuNPs were not stable; resulting in a blue aggregation of AuNPs.

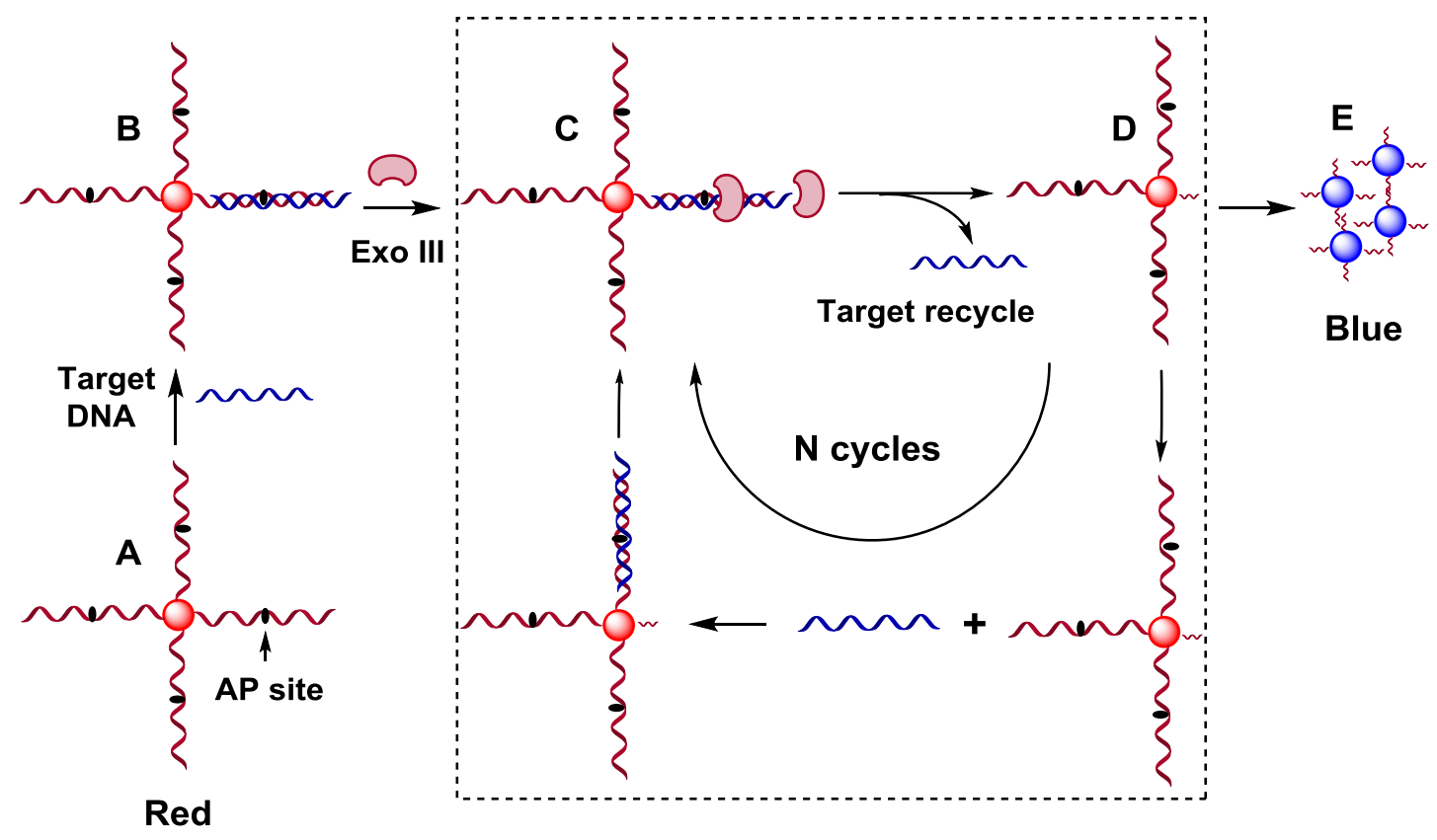

Figure 43. Schematic representation of target recycling in colorimetric assay of exonuclease III: (A) Probe DNA with AP site on the surface of the AuNP with characteristic red color (B) duplex DNA on the surface of AuNP, (C) Exo III recognizes both AP as well as 3' to 5' exonuclease site, (D) probes are cleaved from the surface of AuNP by exonuclease III (E) most of the probes are cleaved on the surface of AuNP resulting aggregation of AuNPs.

Thus, the aggregation of gold nanoparticle is used as an indicator to monitor exonuclease reaction on DNA-modified AuNPs. The extent of exonuclease kinetics can be monitor by measuring the degree of aggregation of AuNPs. The DNA on the surface of gold nanoparticles acts as an electrostatic and steric stabilizer. Therefore, DNAmodified AuNPs are in the red color with a peak at $~ 520 \mathrm{~nm}$. The removal of DNA probe from the surface of the gold nanoparticle destabilized and increased the tendency of aggregation of gold nanoparticles. Therefore, the digested AuNPs produced a blue 
aggregation with a characteristic peak at $\sim 650 \mathrm{~nm}$. Therefore, the ratio of A650/ A520 was taken as a degree of AuNP aggregation.

\subsubsection{DTT treatment of the DNA-AuNP probe}

The activity of enzyme is significantly different between surface-phase reaction and solution-solution reaction. The hybridization of the target on AuNP-conjugated DNA probe is possibly inhibited by non-specific adsorption of DNA on the surface, unfavorable confirmation of DNA probe on the surface and limited space for DNA binding on the gold nanoparticle surface. To solve these problems, we used a thiolated small molecule dithiothreitol (DTT) as back-filler to remove non-specific DNA and reduce DNA surface coverage on the surface of the gold nanoparticle, facilitating favorable DNA hybridization on the nanoparticle surface. We further investigated the kinetics of the enzyme using the DTT-treated AuNPs.

\subsubsection{DTT as a back-filler}

Alkanethiols such as mercaptohexanol $(\mathrm{MCH})$, mercaptoethanol (MCE) and DTT (dithiothreitol) have been widely used to immobilize on the gold surface to form selfassembly monolayer (SAM) to lift DNA probe for enhanced DNA hybridization

efficiency. ${ }^{262,263}$ This backfilling of alkanethiols ensures the vertical orientation of the thiolated DNA probe and also reduces non-specific DNA adsorption and DNA surface coverage. It has been proven that the back-filling of DTT on the surface of the gold electrode is better than that of $\mathrm{MCH}^{264}$ Although such back-filling of the alkanethiols is straight forward on electrode surfaces, it is somehow challenged for the surface of AuNPs due to the poor stability of gold nanoparticle at low DNA surface coverage. Recently, Zhao et al. ${ }^{161}$ have used a low concentration of $\mathrm{MCH}$ as a back-filler to increase DNA hybridization efficiency on AuNPs. 


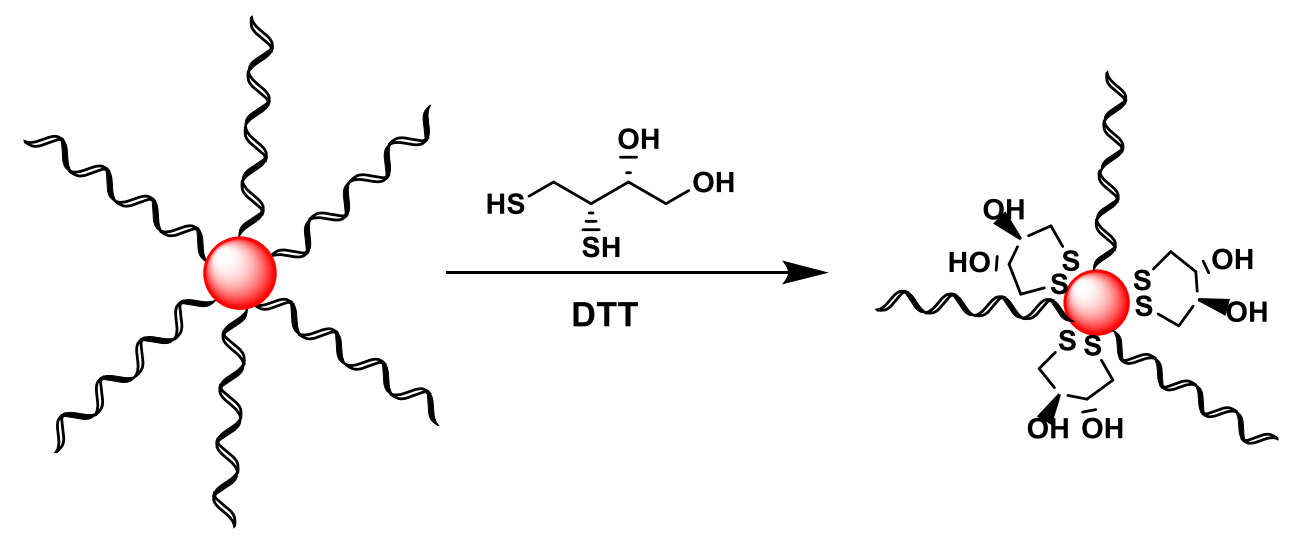

Figure 44. DTT as a back-filler for controlling DNA surface coverage on the DNAmodified AuNPs.

In this study, we used DTT as new back filler to control DNA surface coverage on AuNP surface (Figure 44). DTT is a strong reducing agent with a redox potential of $0.33 \mathrm{~V}$ at $\mathrm{pH} 7{ }^{265}$ The reduction of disulfide bond proceeds by two subsequent thioldisulphide exchange reactions, resulting in the cyclic structure on the surface of the AuNPs. A very high concentration of DTT, typically in molar range, has been used to remove DNA probe from the AuNP ${ }^{151}$ by ligand displacement reaction due to its higher affinity to the gold surface as compared to the thiol modified DNA. To partially remove the probe DNA on from the modified AuNP, we have used very low concentration of DTT in $\mu \mathrm{M}$ range to control DNA surface coverage on the surface of the gold nanoparticle. We hypothesize that it works as a backfiller to change the horizontal or tilted orientation of DNA strands attached to gold nanoparticle into the vertical ones. During the reaction, it may also remove most non-specifically adsorb and some specifically adsorbed DNA from the surface. 


\subsubsection{Kinetics of DTT-displacement for DNA-modified AuNPs}

To find out whether DTT reduced the DNA surface coverage on the surface of the modified AuNPs, we carried out fluorescence experiments using the AuNP-conjugated FAM-labeled DNA probe. Figure 45 represents the kinetics of the FAM-labeled probe modified AuNPs treated with different concentrations of DTT. Although fluorescence of the FAM probe is quenched by AuNPs, we were able to monitor the fluorescence change of the modified AuNPs with different concentration of DTT.

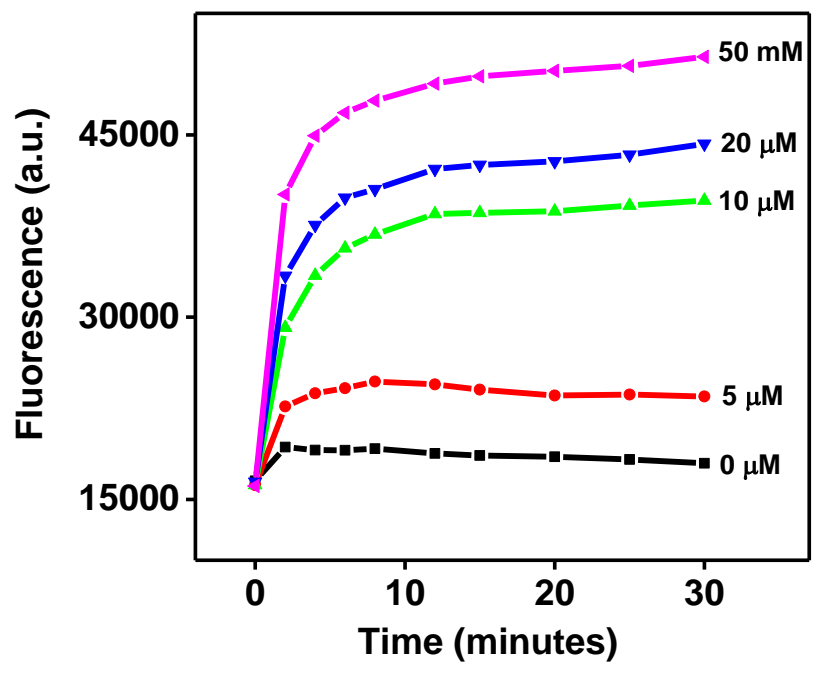

Figure 45. Kinetics of displacement of FAM-labeled DNA from the surface of the gold nanoparticle by DTT.

In the absence of DTT, there was small fluorescence change of about 3000 a.u. during the measurement period, indicating the addition of buffer might have small effect on FAM fluorescence. However, with the addition of DTT solution from $5 \mu \mathrm{M}$ to $50 \mu \mathrm{M}$, the fluorescence of the solution increased, indicating that DTT either changed orientation or/and removed FAM-labeled DNA from the AuNP surface. Both the change in orientation and release of FAM-labeled DNA into the solution increased the fluorescence due to a decreased quenching efficiency of AuNPs. 


\subsubsection{Effect of DTT treatment on surface coverage of the DNA-modified AuNPs}
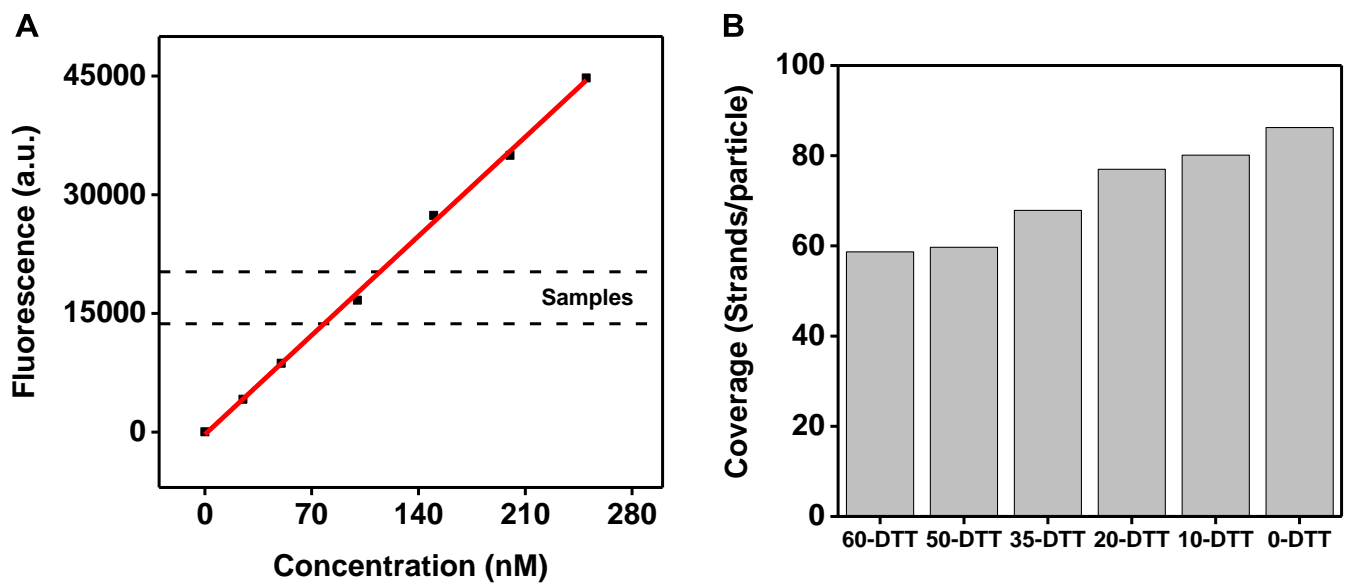

Figure 46. Effect of DTT treatment on the surface coverage of DNA on the surface of AuNP. (A) Calibration curve to determine the change in surface coverage with different concentration of DTT and (B) change in surface coverage as a function of DTT treatment.

To find out the change of DNA surface coverage after the treatment with low concentration of DTT, we separated the DTT-treated AuNPs by washing and centrifugation as described in the procedure. The DNA surface coverage of DTT-treated AuNPs was determined by completely removing DNA from the surface of the gold nanoparticle using the high concentration of DTT $(0.5 \mathrm{M})$. Figure $46 \mathrm{~A}$ represents a calibration curve of standard 1X_FAM probe solutions in the range of 0 to $250 \mathrm{nM}$ with linear regression equation of $Y=179.04 C(n M)-298.98(r=0.9994)$. Using this established calibration curve, we calculated the DNA surface coverage of different DTTtreated samples under same conditions (Figure 46B). The surface coverage of 0, 20, 35, 50, $60 \mu \mathrm{M}$ DTT-treated samples was found to be 86, 80, 77, 68, 60, 59 DNA strand per AuNP which is equivalent to $26.9,25.0,24.0,21.2,18.7$ and $18.4 \mathrm{pmole} / \mathrm{cm}^{2}$, respectively. Clearly, the DNA surface coverage decreased from its initial value (86 DNA strand per AuNP) to 59 DNA strand per AuNP with $60 \mu \mathrm{M}$ DTT treatment for 30 minutes. 


\subsubsection{Activity of Exonuclease III on DTT-treated DNA-modified AuNPs}

\subsubsection{Effect of DTT treatment on the activity of Exonuclease III}

To find the effect of DTT treatment on the activity of Exonuclease III, we treated the DNA-modified AuNPs with DTT concentration in the range of 0 to $60 \mu \mathrm{M}$ range for 30 minutes. We found that the DTT treatment above $60 \mu \mathrm{M}$ concentration produced DNAmodified AuNPs that were not stable in our high salt buffer (10 mM Tris-acetate, $50 \mathrm{mM}$ potassium acetate, $20 \mathrm{mM} \mathrm{CaCl}_{2}, 25 \mathrm{mM} \mathrm{NaCl}$ and $3 \mathrm{mM} \mathrm{MgCl} 2$ ). The activity of Exo III was determined by monitoring the rate of aggregation (the absorbance ratio between $650 \mathrm{~nm}$ and $520 \mathrm{~nm}$ ) of DTT-treated AuNPs in the presence and absence of target. Figure 47 represents the kinetics of the exonuclease III reaction on DNA-modified AuNPs treated with different DTT concentrations.
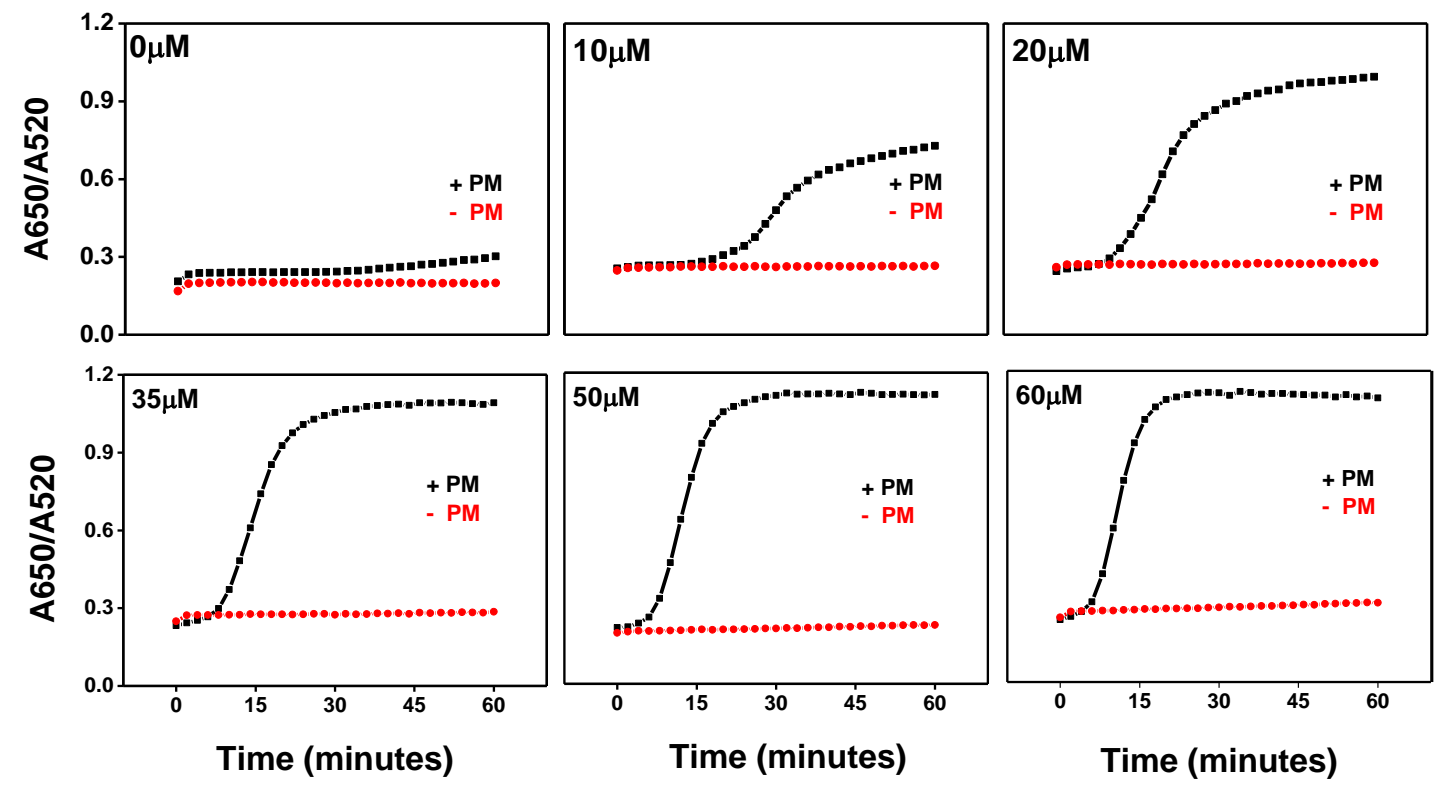

Figure 47. Increase in specificity of exonuclease III by treating with different concentrations of DTT in the reaction buffer containing $10 \mathrm{mM}$ tris acetate $\mathrm{pH} 7.9,50$ $\mathrm{mM}$ potassium acetate, $25 \mathrm{mM} \mathrm{NaCl}, 20 \mathrm{mM} \mathrm{CaCl}_{2}$ and $3 \mathrm{mM} \mathrm{MgCl}_{2}$. The reaction was carried out with 0.02 units $/ \mu$ l of exo III with $100 \mathrm{nM}$ target DNA. 
It was found that the activity of Exo III increased with the increase of DTT concentration in a one-hour reaction time. The enzyme kinetics in the absence of target was very similar for all DTT-treated AuNPs as indicated by constant absorbance ratio of $\sim 0.25$. It means that effect of DTT treatment has no effect on the kinetics of exonuclease III in the absence of target. However, the remarkable difference of exonuclease III kinetics in the presence of target indicated that the activity of Exonuclease III increased with the increase of DTT concentration. After a 60-minute reaction, the difference of absorbance ratio increased from 0.10 in the sample without DTT treatment to 0.79 with $60 \mu \mathrm{M}$ DTT treated sample (Figure 47). This is possibly attributed to more efficient hybridization of target DNA with probe DNA and more active Exo III digestion on the surface of the AuNPs treated with DTT.

\subsubsection{Effect of DTT treatment on rate of Exo III catalyzed reaction}

We also studied the effect of DTT treatment on rate of Exonuclease III-catalyzed reaction.
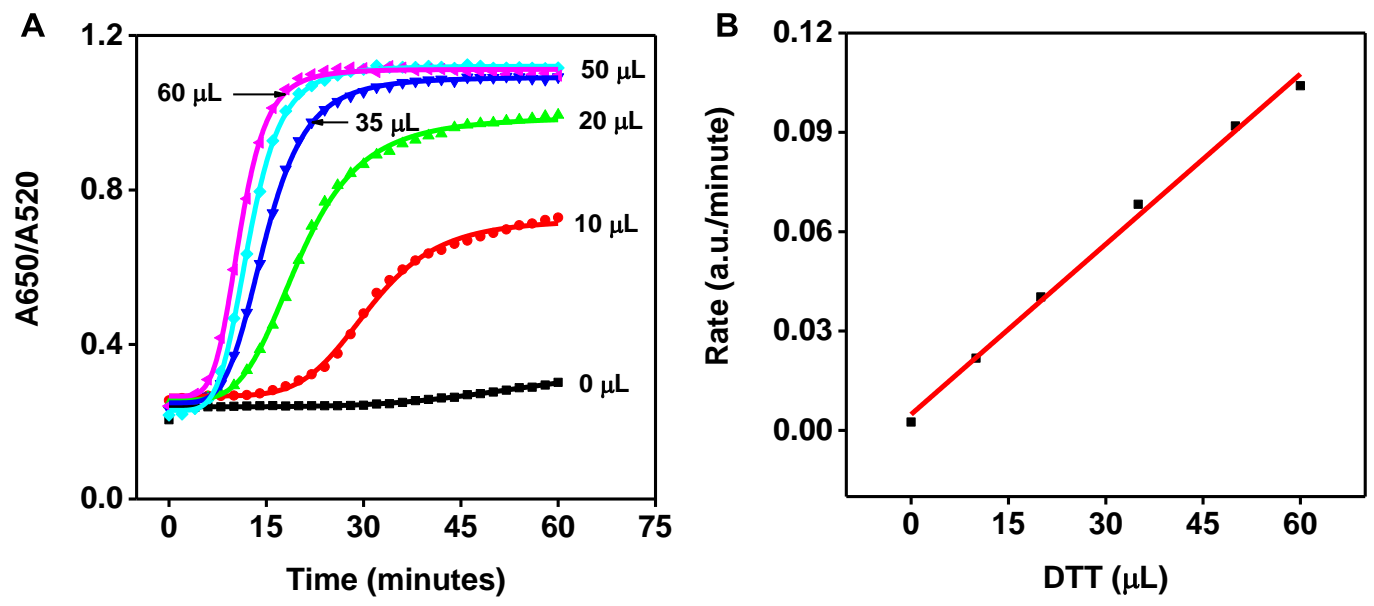

Figure 48. Kinetics of exonuclease III on DTT treated DNA-AuNP probes: $(A)$ kinetics of

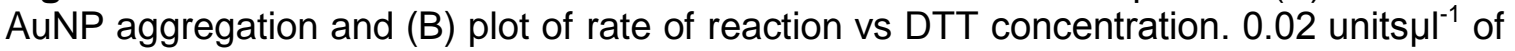
exonuclease III was used in the presence of $100 \mathrm{nM}$ target DNA, with the DTT reaction of probe-AuNPs for 30 minutes in the concentration range of 0 to $60 \mu \mathrm{M}$. 
Figure 48 represents the kinetics of exonuclease III on the DNA-modified AuNPs treated with different concentrations of DTT in the range of 0 to $60 \mu \mathrm{M}$ for 30 minutes. It was found that rate of Exo III digestion increased with the increase of DTT concentration. Figure 48A represents the relationship between the rate of exonuclease III-catalyzed reaction and the concentration of DTT employed in the experiment. We observed that the rate of reaction increased from 0.0025 per minute with the probe without DTT treatment to 0.1040 per minute with $60 \mu \mathrm{M}$ DTT treated AuNPs (Figure 48B).

\subsubsection{Effect of concentration of target DNA on reaction rate of Exo III-catalyzed} reaction

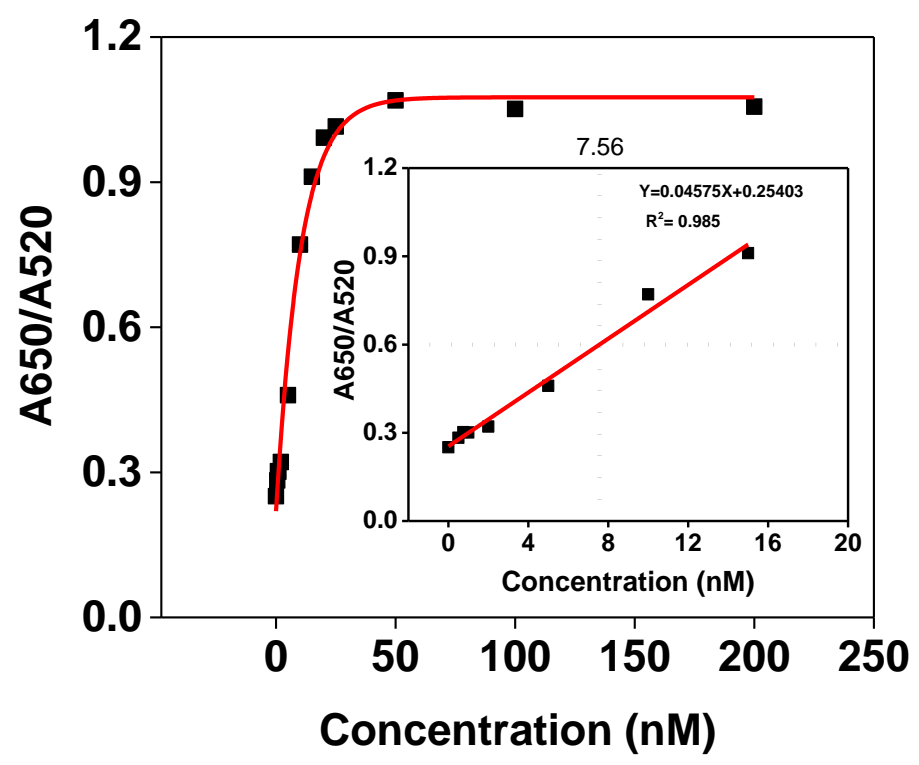

Figure 49. Effect of target concentration on the rate of exonuclease catalyzed reaction: The plot of the rate of aggregation (absorbance ratio) against the target concentration in the range of 0 to $200 \mathrm{nM}$. $60 \mu \mathrm{M}$ DTT treated probe-AuNP substrate was mixed with the different target concentrations in $10 \mathrm{mM}$ Tris-acetate buffer at $\mathrm{pH} 7.9$ containing $50 \mathrm{mM}$ potassium acetate, $20 \mathrm{mM} \mathrm{CaCl}_{2}, 25 \mathrm{mM} \mathrm{NaCl}$ and $3 \mathrm{mM} \mathrm{MgCl}_{2}$ before reacting with 0.2 units $/ \mu$ for 30 minutes. 
We used the DTT-treated AuNPs to study the effect of target concentration on the reaction rate of Exo III-catalyzed reaction. The concentration of target DNA was varied from 0 to $200 \mathrm{nM}$, with optimum DTT-treated DNA-modified AuNPs. Figure 49 represents the plot of absorption ratio of the modified AuNPs with different concentration of targets and 0.02 units/ $\mu$ l exonuclease III after a 30-minute reaction. Experimental results demonstrated that the aggregation rate of AuNPs increased with the increase of target concentration from 0 to $50 \mathrm{nM}$. With further increase in target concentration, the rate remained practically constant as shown in Figure 49. Noted that the rate of reaction increased linearly from 0 to $15 \mathrm{nM}$ as shown in the inset of Figure 49 . When the absorbance ratio of $\sim 0.6$ was used as a threshold value for a clear difference in color from red to blue, a concentration of $~ 7.56 \mathrm{nM}$ was the minimum target concentration that can be observed by naked eye.

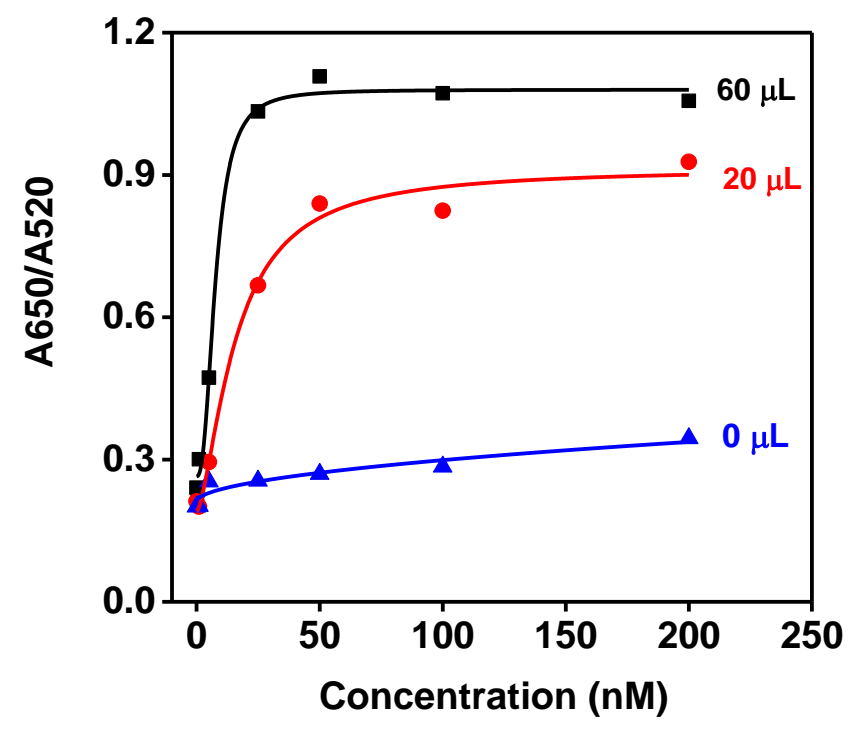

Figure 50. Effect of DTT treatment on target concentration on the rate of exonuclease catalyzed reaction: The plot of rate of aggregation (absorbance ratio) against the target concentration in the range of 0 to $250 \mathrm{nM}$. 0, 20, $60 \mu \mathrm{M}$ DTT treated probe-AuNP substrates were mixed with the different target concentrations in $10 \mathrm{mM}$ Tris-acetate buffer at pH 7.9 containing $50 \mathrm{mM}$ potassium acetate, $20 \mathrm{mM} \mathrm{CaCl}_{2}, 25 \mathrm{mM} \mathrm{NaCl}^{2}$ and 3 $\mathrm{mM} \mathrm{MgCl}_{2}$ before reacting with 0.2 units $\mu^{-1}$ for 30 minutes. 
We further compared the exonuclease activity on different DTT-treated, DNAmodified AuNP. We found that different DTT-treated AuNPs produced different target dependency of exonuclease reaction. Figure 50 represents the comparison of the calibration curves of different DTT-treated DNA-modified AuNPs. The absorbance ratio of DNA-modified AuNPs without DTT treatment remained practically constant in the target concentration range from 0 to $250 \mathrm{nM}$. However, the absorbance ratio of both 20 and $60 \mu \mathrm{M}$ DTT-treated, AuNPs increased with the increase of target concentration from $\sim 0.25$ to $\sim 0.92$ and $\sim 0.25$ to $\sim 1.12$ respectively, indicating DTT treatment greatly enhanced exonuclease III activity.

For $20 \mu \mathrm{M}$ DTT-treated, DNA-modified AuNPs, the absorbance ratio increased up to 0.9 with an increase of target concentration from 0 to $200 \mathrm{nM}$. However, for $60 \mu \mathrm{M}$ DTTtreated DNA-modified AuNPs, the absorbance ratio increased up to 1.1 in concentration range of 0 to $50 \mathrm{nM}$ of target and remains constant on further increase in target concentration up to $200 \mathrm{nM}$. The difference in exonuclease kinetics can be explained by higher enzyme efficiency in DTT-treated samples as compared to the untreated sample. This is attributed to better hybridization efficiency of target DNA, high Exo III activity and faster aggregation of AuNPs in DTT-treated samples due to lower DNA surface coverage on the AuNP surface.

\subsubsection{Performance of newly designed DNA probe}

To compare the performance between newly designed $1 \mathrm{X}$ probe for the dual activity of Exonuclease III and traditional OX probe for 3' to 5' exonuclease activity, we performed the Exo III based colorimetric assay under same experimental conditions with two different DNA-modified AuNPs that had similar surface density of $105 \pm 4$ and $90 \pm 5$ Figure 51 represents the comparison of assay performance of two different DNAmodified AuNPs. The comparison of absorbance ratio between the presence and 
absence of target shows that newly designed probe was more specific. The difference of absorbance ratio was $\sim 0.74$ for $1 \mathrm{X}$ probe as compared to just $\sim 0.35$ for $0 \mathrm{X}$ probe after 60 minutes of reaction time. Similarly, the comparison of the reaction rate of two different probes demonstrated that faster red-to-blue color change could be obtained in the newly designed $1 \mathrm{X}$ probe as compared to the traditional $\mathrm{OX}$ probe.
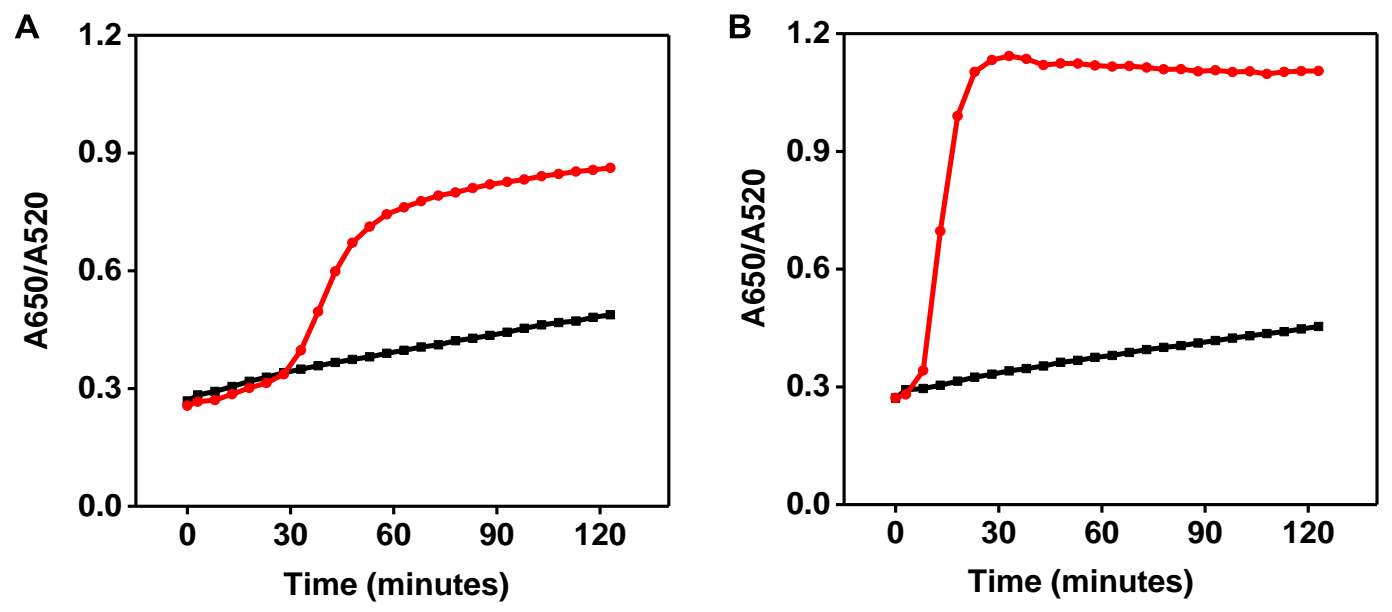

Figure 51. Comparison of performance of newly designed probe with the dual activity of exonuclease III (1X) with the traditional probe with 3' to 5' exonuclease activity (OX): the kinetics of exonuclease III reaction for $(A) 0 X$ and $(B) 1 X$ probe. The reaction was carried out using 0.2 units/ $\mu \mathrm{l}$ of Exo III and $100 \mathrm{nM}$ target DNA in $10 \mathrm{mM}$ tris acetate buffer at $\mathrm{pH}$ 7.9 containing $50 \mathrm{mM}$ potassium acetate, $20 \mathrm{mM} \mathrm{CaCl}_{2}, 25 \mathrm{mM} \mathrm{NaCl}$ and $3 \mathrm{mM} \mathrm{MgCl}_{2}$. Both DNA probes were treated with optimized dithiothreitol concentration before the exonuclease assay.

As a result, the color change from red to blue was observed in $\sim 9$ minutes in the $1 \mathrm{X}$ probe as compared to $\sim 45$ minutes in the $0 \mathrm{X}$ probe. When the probe was hybridized with the target, the newly designed $1 \mathrm{X}$ probe was a substrate for both AP endonuclease and 3' to 5' exonuclease activities. Exo III utilized its AP endonuclease activity to cleave the abasic site incorporated into the probe, forming a new 3' OH group for Exo III to employ its exonuclease activity. 3' to 5' exonuclease activity now acted on two separate positions of duplex DNA to perform digestion, releasing mononucleotides from two 
different positions that remarkably increased the rate of cleavage of probe DNA from the surface of AuNPs.

\subsubsection{Exonuclease assay based on DTT-treated DNA-modified AuNPs}

To apply DTT-treated DNA-modified AuNPs for the colorimetric assay of the activity of exonuclease III, we modified the unlabeled 1X_NoFAM probe on the AuNPs.

\subsubsection{Modification and characterization of unlabeled DNA probes on AuNPs}

Figure 52 represents the schematic of DTT displacement assay for the determination of DNA concentration on the surface of AuNP. The thiol modified DNA was displaced from the surface of AuNP using ligand exchange reaction and separated from gold nanoparticle using centrifugation. The fluorescence of released DNA was then measured by using oligreen dye and compared with the calibration curve using standard DNA solution.
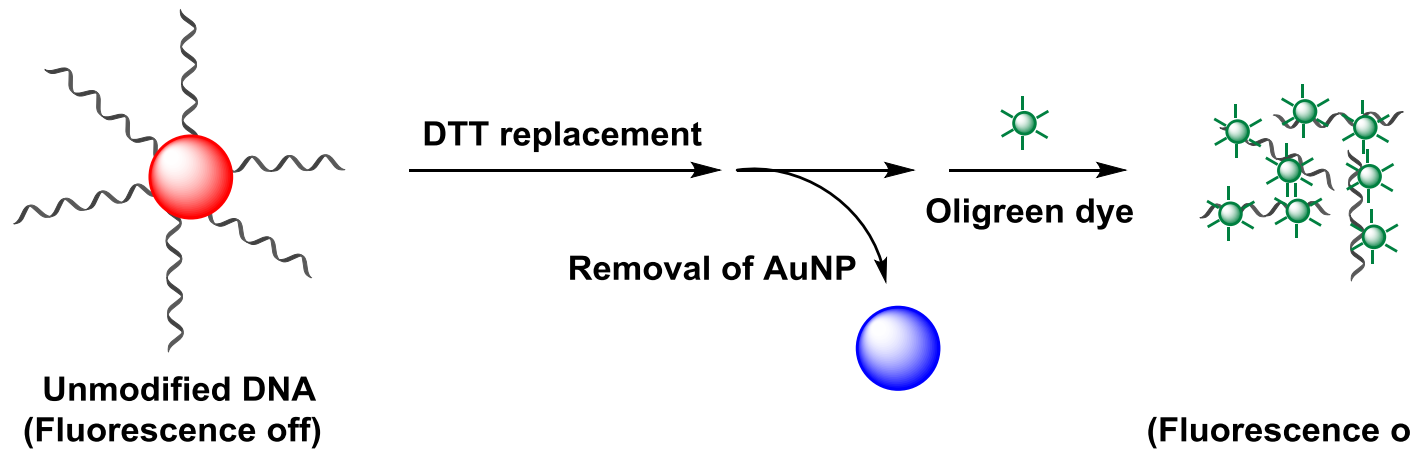

Figure 52. Schematic representation of DTT displacement fluorescence assay for the determination of the concentration of fluorescence labeled DNA on the surface of AuNP.

Figure 53 represents the calibration curve of unmodified 1X_NoFAM probe in the range of 0 to $150 \mathrm{nM}$ using oligreen assay. To avoid the fluorescence change upon addition of DTT, the same DTT solution $(0.5 \mathrm{M})$ was used for both preparations of standard curve and removal of thiol DNA from the AuNP surface. The concentration of DNA on the surface of AuNP was determined using the established calibration curve 
with a linear regression equation $(y=131.26 X+343)$. The surface coverage was determined by dividing concentration of the DNA immobilized on AuNP by the concentration of AuNP (71.68 nM) and was found to be $68 \pm 5$ DNA strands per AuNP $\left(21.2 \pm 1.5\right.$ pmole $\left.\mathrm{cm}^{-2}\right)$.

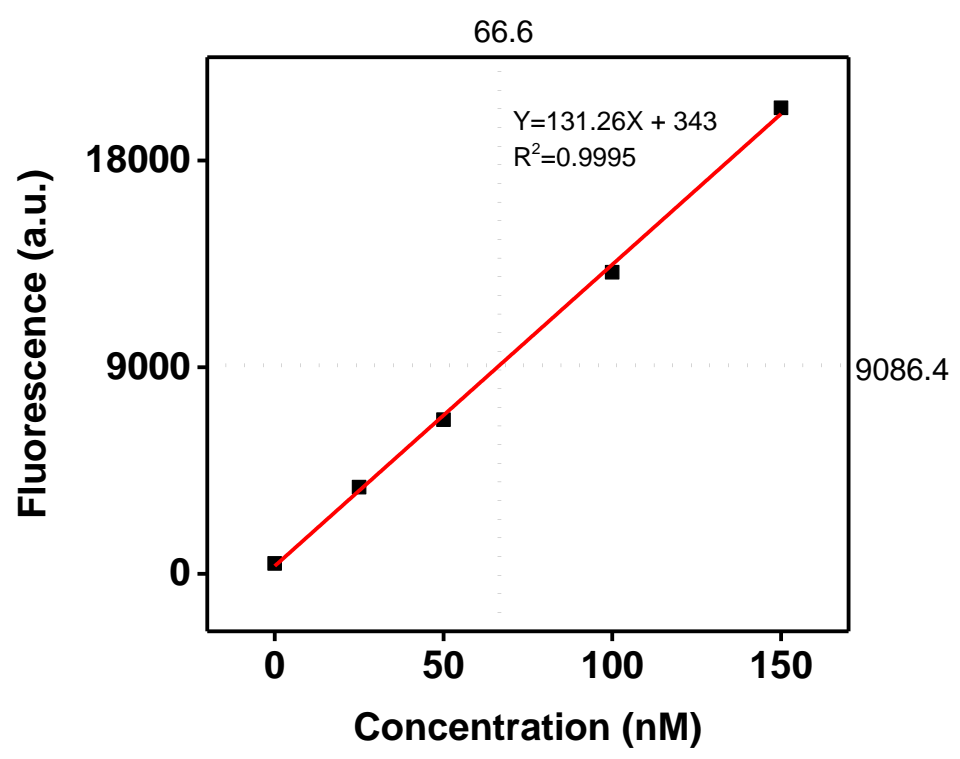

Figure 53. Calibration curve of of DTT treated 1X_NoFAM probe in the range of 0 to 150 $\mathrm{nM}$ for the determination of surface coverage by DTT displacement method. The DNA concentration on DTT treated samples were determined by using oligreen assay.

\subsubsection{2. $\mathrm{NaCl}$ concentration for optimizing sensor performance}

Sodium chloride is essential in our Exo III digested colorimetric assays involving DNA-modified AuNPs. It is necessary not only to increase DNA hybridization efficiency on the nanoparticle surface but also is required to produce a salt-induced aggregation of AuNPs. In order to find out whether $\mathrm{NaCl}$ is required for detecting the activity of Exo III using our DTT-treated DNA-modified AuNPs, we performed experiments with $6 \mathrm{mM}$ concentration of $\mathrm{MgCl}_{2}$ and different concentrations of $\mathrm{NaCl}$ in the range of 0 to $100 \mathrm{mM}$. Figure 54 represented the effect of $0(A), 25(B), 50(C), 100(D) \mathrm{mM} \mathrm{NaCl}$ concentration in 
the presence and absence of target. In the absence of target, there is negligible effect of $\mathrm{NaCl}$ indicated by constant absorbance ratio. However, in the presence of target, the absorbance ratio decreased with the increase of $\mathrm{NaCl}$ concentration (Figure 54E).
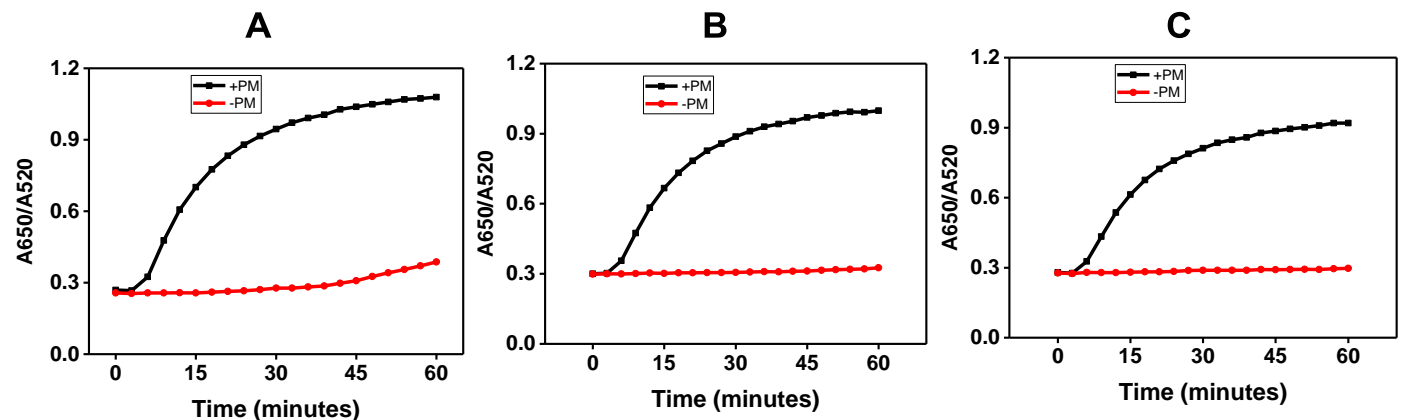

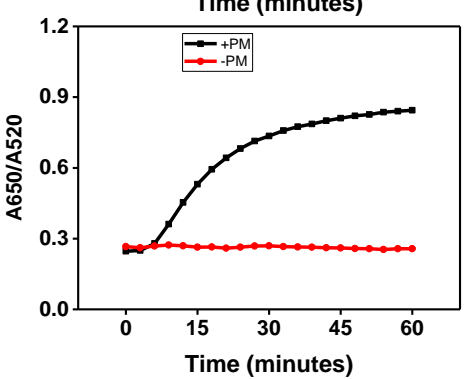

D

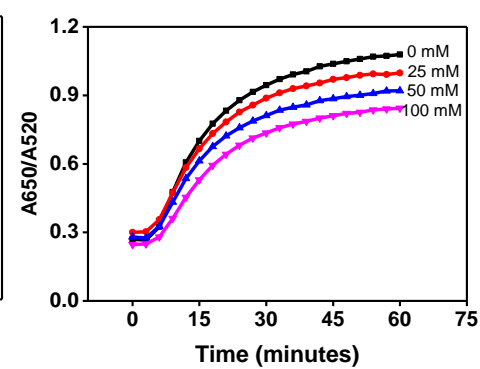

E

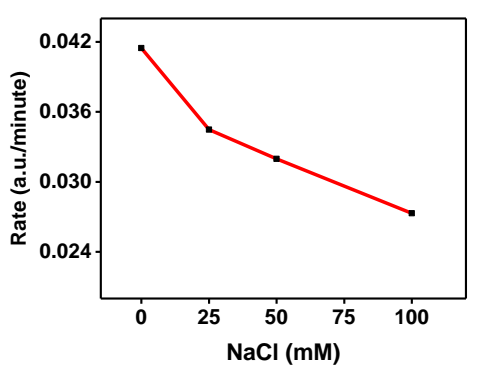

$\mathbf{F}$

Figure 54. Effect of different concentration of $\mathrm{NaCl}(0,25,50$ and $100 \mathrm{mM})$ on the activity of Exonuclease III on DTT treated gold nanoparticle substrate in the presence (+) and the absence (-) of target DNA (A, B, C and D respectively). Comparison of change in absorbance ratio (E) and rate of reaction (F) in different concentration of $\mathrm{NaCl}$ in the presence of $50 \mathrm{nM}$ of target DNA in $10 \mathrm{mM}$ Tris-acetate buffer $\mathrm{pH} 7.9$ containing $6 \mathrm{mM}$ of $\mathrm{MgCl}_{2}$.

The relationship between rate constant and $\mathrm{NaCl}$ concentration indicated that the aggregation rate of AuNPs decreased with the increase of $\mathrm{NaCl}$ concentration. This is due to the decreased activity of Exonuclease III with the increase of salt concentration, which is consistent with the previous reports. ${ }^{182}$ Therefore, we did not use $\mathrm{NaCl}$ for our colorimetric exonuclease assay to maximize the assay's sensitivity.

\subsubsection{3. $\mathrm{MgCl}_{2}$ concentration for optimizing sensor performance}

Magnesium chloride is essential for both AP endonuclease and 3' to 5' exonuclease activity of Exonuclease III. ${ }^{176,178,183}$ In order to find the optimum concentration of $\mathrm{MgCl}_{2}$ 
for utilizing dual activities of exonuclease III, we varied the concentration of $\mathrm{MgCl}_{2}$ in the range of 0 to $10 \mathrm{mM}$ and we found that DTT-treated AuNPs were not stable in the buffer containing $\mathrm{MgCl}_{2}$ above $10 \mathrm{mM}$.
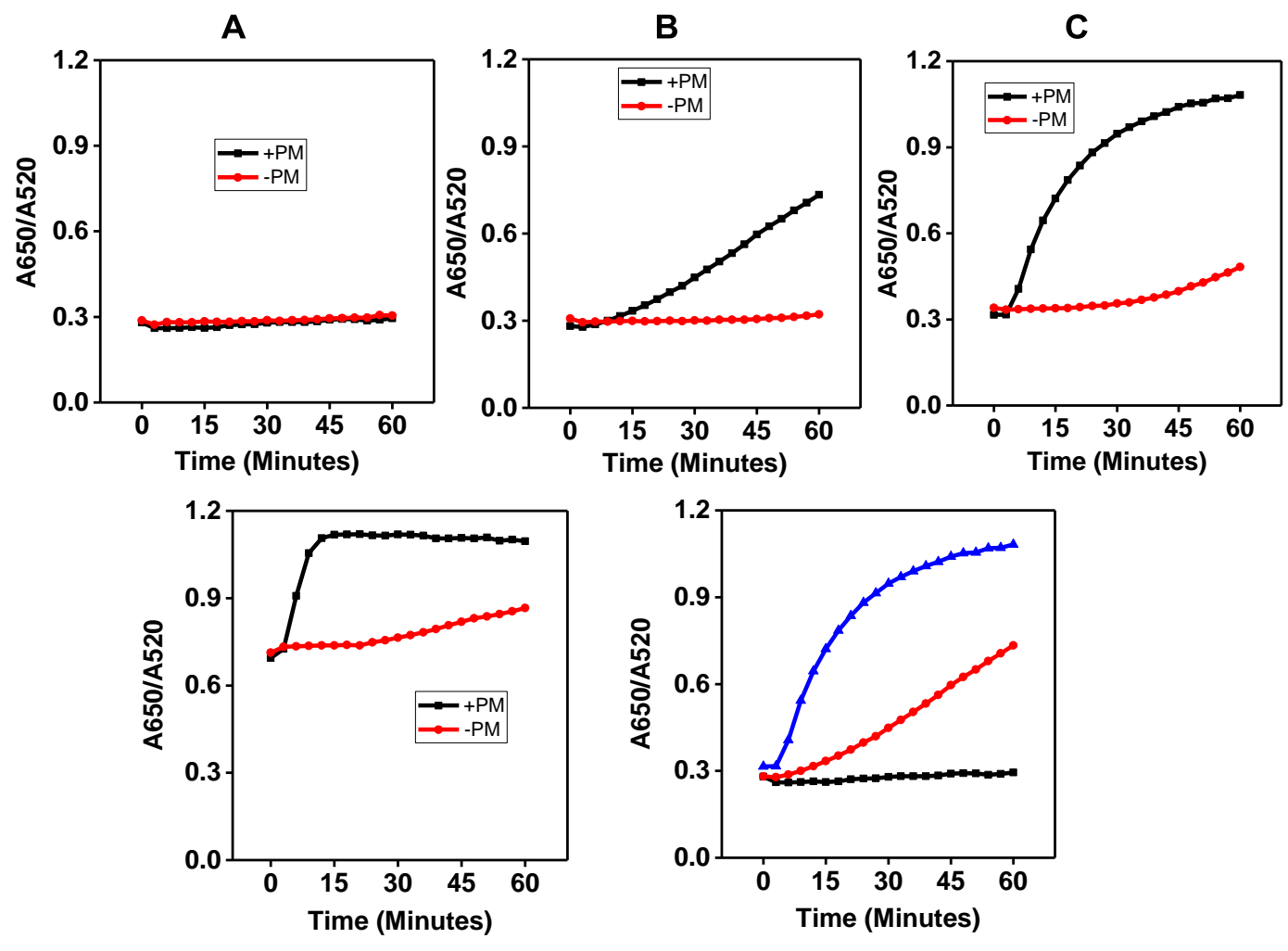

D

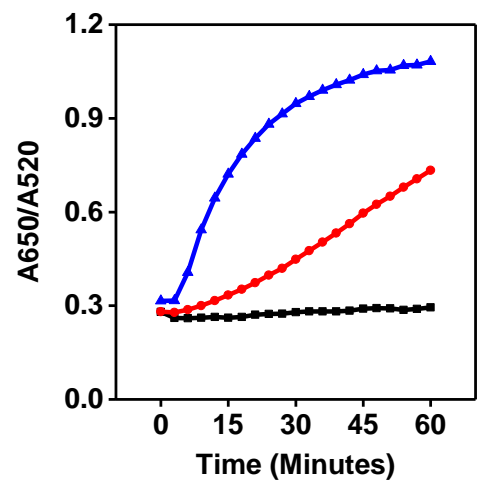

E

Figure 55. Effect of different concentration of $\mathrm{MgCl}_{2}(0,3,6$ and $9 \mathrm{mM})$ on the activity of Exonuclease III on DTT-treated probe-AuNP substrate in the presence $(+)$ and in the absence (-) of target DNA (A, B, C and D respectively). Comparison of rate of reaction (E) in different concentration of $\mathrm{MgCl}_{2}$ in the presence of $50 \mathrm{nM}$ of target DNA in $10 \mathrm{mM}$ Tris-acetate buffer at $\mathrm{pH}$ 7.9.

Figure 55 represented the effect of $\mathrm{MgCl}_{2}$ concentration on Exonuclease activity. In the absence of $\mathrm{MgCl}_{2}$, there was no activity of exonuclease III, indicating that $\mathrm{MgCl}_{2}$ is essential for activating exonuclease activity. The exonuclease reaction rate increased with the increase of $\mathrm{MgCl}_{2}$ concentration (Figure 55E). However, with the higher concentration of $\mathrm{MgCl}_{2}(10 \mathrm{mM})$, the specificity of Exo III was lost as indicated by the smaller difference of absorbance ratio between with and without target $(\sim 0.3)$ (Figure 
55D) as compared to the difference in absorbance ratio in the presence of $6 \mathrm{mM} \mathrm{MgCl}_{2}$

( 0.6). Therefore, $6 \mathrm{mM}$ of $\mathrm{MgCl}_{2}$ was used as the optimum concentration for generating fast and specific exonuclease III reaction.

\subsubsection{Colorimetric exonuclease assay based on DTT-treated DNA-modified}

\section{AuNPs}

Figure 56 represents the relationship between the absorbance ratio and different concentrations of exonuclease $\mathrm{III}$ in the range of 0 to 100 units/ $\mathrm{ml}$ after a 15-minute exonuclease III reaction.

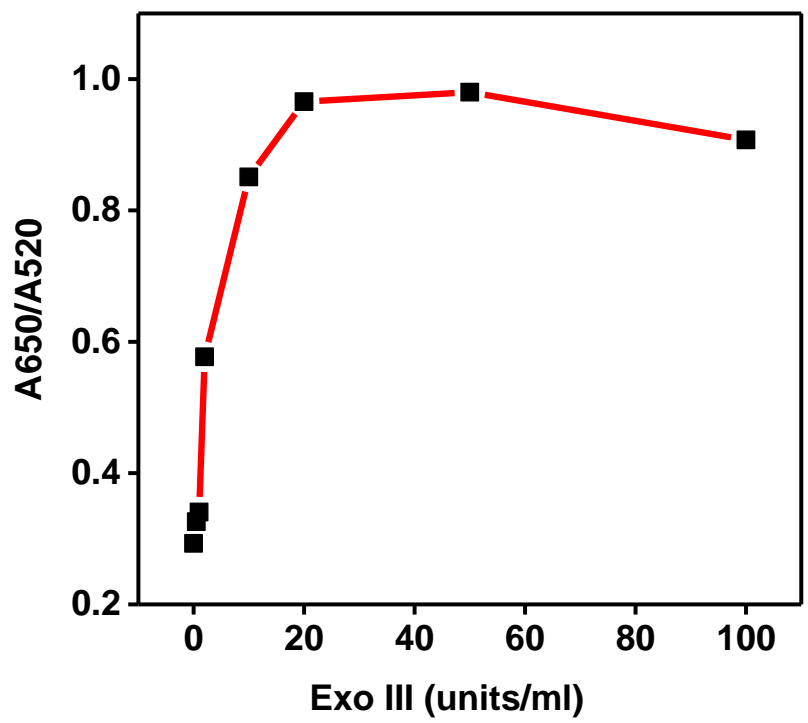

Figure 56. The plot of the ratio of absorbance against different concentration of exonuclease $\mathrm{III}$ in the range of 0 to $100 \mathrm{units} / \mathrm{ml}$. The absorbance ratio was measured after 15 minutes of the reaction of different concentrations of exonuclease III with DTTtreated 1X probe DNA on the surface of the gold nanoparticle in the presence of $50 \mathrm{nM}$ of target DNA in $10 \mathrm{mM}$ Tris-acetate buffer $\mathrm{pH} 7.9$ containing $6 \mathrm{mM}$ of $\mathrm{MgCl}_{2}$.

The aggregation rate of AuNPs, as indicated by absorbance ratio, increased with the increase of exonuclease III concentration, suggesting that aggregation of AuNPs is directly related to exonuclease activity. The absorbance ratio increased with the increase 
of exonuclease concentration and became saturated when 20 units $/ \mathrm{ml}$ or higher of Exonuclease III was used. Clearly, as low as 2 units $/ \mathrm{ml}$ of exonuclease III could be detected by naked eye.

\subsection{Broader Impacts}

We have developed a new colorimetric assay based on a DNA substrate for detecting DNA and exonuclease activity. We were able to enhance the rate of exonuclease reactions on AuNP-conjugated DNA probes by designing new probe and by reducing the surface coverage of DNA on the surface of AuNPs using DTT as a backfiller. We were able to lower the detection time from several hours to 15 minutes by lowering the surface coverage of DNA-modified gold nanoparticles by DTT treatment. The developed method was able to detect as low as $8 \mathrm{nM}$ of DNA and 2 units $/ \mathrm{ml}$ of exonuclease III within 15 minutes by the naked eye, which is significant for point of care applications. The current colorimetric assay could be used to screen the inhibitors of class II AP endonucleases in a high-throughput fashion to develop and design inhibitors for the predictive and prognostic study of cancer markers. The detection platform could be extended to other DNA probes or aptamers for fast and sensitive detection of any other disease markers or small molecules. We envision that development of transferring the solution-based method into paper substrate could lead to the paper-based colorimetric sensor for point of care diagnostics in the future. 


\section{Chapter 6. Summary and Future work}

\subsection{Summary}

This dissertation describes the fabrication, characterization, and application of simple, cheap, and efficient nanomaterial-based platforms for electrochemical and colorimetric sensing applications. This work focused on the use of the excellent electrochemical properties of CNTs for paper-based electrodes and the sensitive optical properties of AuNPs for colorimetric detection. First, we demonstrated the formation of films comprising low-density, three-dimensional, porous, interconnected networks of CNTs on paper by vacuum filtration of well-dispersed CNTs, and demonstrated the use of such films as electrochemical sensors for the sensitive detection of guanine-based nucleotides. Second, we assembled three-dimensional, porous Pt networks on top of a conducting CNT support through vacuum-assisted assembly and demonstrated the use of these hybrid films as an electrocatalyst for methanol oxidation. Finally, we used DTT to modulate DNA surface coverage of modified AuNPs, which we used for sensitive and fast detection of DNA and enzymes.

CNTs have been used in the past as a modifier to increase electron transfer rates on different electrode surfaces, and in Chapter 3 we demonstrated the use of paper as a substrate for CNT-based electrodes. The resulting porous, uniform, well-dispersed, conductive CNT network is an excellent alternative to traditional CNT-based electrodes. We found that the optimum surface density of CNTs is very important in terms of performance as an electrode material, strongly influencing both conductance and electron transfer rates. By lowering the CNT surface density, we were able to decrease background current, enabling sensitive detection of guanine-based nucleotides. While the sensitivity is not yet ideal for practical applications, it is comparable to recently reported CNT-modified electrodes with or without other modifying agents. 
Pt-SWCNT composites have been widely used on the surface of a variety of traditional electrodes, and in Chapter 4 we showed that Pt-SWCNT composites could be directly formed on a paper substrate. We further demonstrated the use of paper-based SWCNT thin films prepared by vacuum filtration method as a conductive support for the platinum catalyst. Our paper-based Pt-SWCNT thin film proved to be a much better conducting support compared to the commonly-used classical GC and FTO solid electrodes. As a demonstration of the functionalization of paper-based CNT electrodes, we deposited platinum nanoparticles via both a vacuum filtration method and an electrodeposition method. The Pt-SWCNT composites produced via the vacuum filtration method showed three-dimensional, continuous and porous platinum structure with a relatively narrow size distribution of PtNPs, resulting in the higher electrochemical surface area and electrocatalytic response towards methanol compared to the electrochemically-deposited hybrid films.

It has been demonstrated that the density of thiol-modified DNA on the surface of AuNPs affects the colloidal stability of the particles, the orientation of probe DNA and its hybridization efficiency with the target, and the activity of DNA-digesting enzymes. The combination of all these factors ultimately dictates the reaction time and sensitivity of DNA-AuNP-based colorimetric assays. In Chapter 5, we demonstrated that DTT could be used as a back-filler to modulate the DNA density on the surface of AuNPs. Using this approach, we were able to greatly increase the reaction rate and sensitivity of DNAAuNP colorimetric assays for both DNA and exonuclease III.

\subsection{Future work}

This dissertation demonstrates the electrochemical and colorimetric detection platforms for the development of low-cost, portable and disposable paper-based analytical devices for on-site or point of care applications. The future work involves development of 
methods to incorporate paper-based SWCNT or PtNP-SWCNT electrode material on paper-based devices for the development of low-cost, portable and disposable paperbased sensors. The flexibility of paper or its ease of dissolution could be used to incorporate into the choice of analytical devices. However, possible changes in electrochemical properties during incorporation need to consider. Similarly, more research is required to adopt solution-based colorimetric detection platform on a paper substrate. The incorporation of DNA modified gold nanoparticle on paper substrate, efficiency of DTT treatment, the conformation of DNA on the surface of the gold nanoparticle and the enzyme efficiency need to be considered before development of portable paper-based device for rapid screening of DNA, small molecules or enzyme inhibitors. 


\section{References}

(1) lijima, S.; Ichihashi, T. Single-Shell Carbon Nanotubes of 1-Nm Diameter. Nature 1993, 363, 603-605.

(2) Yang, W.; Ratinac, K. R.; Ringer, S. R.; Thordarson, P.; Gooding, J. J.; Braet, F. Carbon Nanomaterials in Biosensors: Should You Use Nanotubes or Graphene? Angew. Chemie - Int. Ed. 2010, 49, 2114-2138.

(3) Ajayan, P. M. Nanotubes from Carbon. Chem. Rev. 1999, 99, 1787-1799.

(4) Schnorr, J. M.; Swager, T. M. Emerging Applications of Carbon Nanotubes. Chem. Mater. 2011, 23, 646-657.

(5) Neto, A. H. C.; Guinea, F.; Peres, N. M. R.; Novoselov, K. S.; Geim, A. K. The Electronic Properties of Graphene. Rev. Mod. Phys. 2009, 81, 109-155.

(6) Zavislyak, I. V; Popov, M. A. Fundamental Electronic Properties and Applications of Single-Walled Carbon Naotubes. Acc. Chem. Res. 2002, 35, 1018-1025.

(7) McEuen, P. L.; Fuhrer, M. S. Single-Walled Carbon Nanotube Electronics. IEEE Trans. Nanotechnol. 2002, 99, 78-85.

(8) Saito, R.; Dresselhaus, G.; Dresselhaus, M. S. Physical Propeties of Carbon Nanotubes; Imperial College Press: London, U.K., 1998.

(9) Banks, C. E.; Compton, R. G. New Electrodes for Old: From Carbon Nanotubes to Edge Plane Pyrolytic Graphite. Analyst 2006, 131, 15.

(10) Holloway, A. F.; Wildgoose, G. G.; Compton, R. G.; Shao, L.; Green, M. L. H. The Influence of Edge-Plane Defects and Oxygen-Containing Surface Groups on the Voltammetry of Acid-Treated, Annealed and "super-Annealed" Multiwalled Carbon Nanotubes. J. Solid State Electrochem. 2008, 12, 1337-1348.

(11) Gong, K.; Chakrabarti, S.; Dai, L. Electrochemistry at Carbon Nanotube Electrodes: Is the Nanotube Tip More Active than the Sidewall? Angew. Chemie Int. Ed. 2008, 47, 5446-5450.

(12) Hirsch, A. Functionalization of Single-Walled Carbon Nanotubes. Angew. Chemie - Int. Ed. 2002, 41, 1853-1859. 
(13) Niyogi, S.; Hamon, M. A.; Hu, H.; Zhao, B.; Bhowmik, P.; Sen, R.; Itkis, M. E.; Haddon, R. C. Chemistry of Single-Walled Carbon Nanotubes. Acc. Chem. Res. 2002, 35, 1105-1113.

(14) Xiao, N.; Venton, B. J. Rapid, Sensitive Detection of Neurotransmitters at Microelectrodes Modified with Self-Assembled SWCNT Forests. Anal. Chem. 2012, 84, 7816-7822.

(15) Goyal, R. N.; Singh, S. P. Simultaneous Voltammetric Determination of Dopamine and Adenosine Using a Single Walled Carbon Nanotube - Modified Glassy Carbon Electrode. Carbon N. Y. 2008, 46, 1556-1562.

(16) Yoshii, K.; Tsuda, T.; Arimura, T.; Imanishi, A.; Torimoto, T.; Kuwabata, S. Platinum Nanoparticle Immobilization onto Carbon Nanotubes Using Pt-Sputtered Room-Temperature Ionic Liquid. RSC Adv. 2012, 2, 8262.

(17) Kaempgen, M.; Chan, C. K.; Ma, J.; Cui, Y.; Gruner, G. Printable Thin Film Supercapacitors Using Single-Walled Carbon Nanotubes 2009. Nano Lett. 2009, 9, 1872-1876.

(18) Lee, J. H.; Kong, B. S.; Yang, S. B.; Jung, H. T. Fabrication of Single-Walled Carbon Nanotube/tin Nanoparticle Composites by Electrochemical Reduction Combined with Vacuum Filtration and Hybrid Co-Filtration for High-Performance Lithium Battery Electrodes. J. Power Sources 2009, 194, 520-525.

(19) Rowell, M. W.; Topinka, M. A.; McGehee, M. D.; Prall, H.-J.; Dennler, G.; Sariciftci, N. S.; Hu, L.; Gruner, G. Organic Solar Cells with Carbon Nanotube Network Electrodes. Appl. Phys. Lett. 2006, 88, 233506.

(20) Girishkumar, G.; Vinodgopal, K.; Kamat, P. V. Carbon Nanostructures in Portable Fuel Cells: Single-Walled Carbon Nanotube Electrodes for Methanol Oxidation and Oxygen Reduction. J. Phys. Chem. B 2004, 108, 19960-19966.

(21) Kong, J.; Cassell, A. M.; Dai, H. Chemical Vapor Deposition of Methane for Single-Walled Carbon Nanotubes. Chem. Phys. Lett. 1998, 292, 567-574.

(22) Unalan, H. E.; Chhowalla, M. Investigation of Single-Walled Carbon Nanotube Growth Parameters Using Alcohol Catalytic Chemical Vapour Deposition. Nanotechnology 2005, 16, 2153-2163. 
(23) Zhang, J.; Zou, H.; Qing, Q.; Yang, Y.; Li, Q.; Liu, Z.; Guo, X.; Du, Z. Effect of Chemical Oxidation on the Structure of Single-Walled Carbon Nanotubes. J. Phys. Chem. B 2003, 107, 3712-3718.

(24) Viet, N. X.; Ukita, Y.; Chikae, M.; Ohno, Y.; Maehashi, K.; Matsumoto, K.; Viet, P. H.; Takamura, Y. Fabrication of New Single-Walled Carbon Nanotubes Microelectrode for Electrochemical Sensors Application. Talanta 2012, 91, 88-94.

(25) Yang, C.; Jacobs, C. B.; Nguyen, M. D.; Ganesana, M.; Zestos, A. G.; Ivanov, I. N.; Puretzky, A. A.; Rouleau, C. M.; Geohegan, D. B.; Venton, B. J. Carbon Nanotubes Grown on Metal Microelectrodes for the Detection of Dopamine. Anal. Chem. 2016, 88, 645-652.

(26) Tang, J. M.; Jensen, K.; Waje, M.; Li, W.; Larsen, P.; Pauley, K.; Chen, Z.; Ramesh, P.; Itkis, M. E.; Yan, Y.; et al. High Performance Hydrogen Fuel Cells with Ultralow Pt Loading Carbon Nanotube Thin Film Catalysts. J. Phys. Chem. C 2007, 111, 17901-17904.

(27) Tsai, M. C.; Yeh, T. K.; Juang, Z. Y.; Tsai, C. H. Physical and Electrochemical Characterization of Platinum and Platinum-Ruthenium Treated Carbon Nanotubes Directly Grown on Carbon Cloth. Carbon N. Y. 2007, 45, 383-389.

(28) Kumar, M.; Ando, Y. Chemical Vapor Deposition of Carbon Nanotubes: A Review on Growth Mechanism and Mass Production. J. Nanosci. Nanotechnol. 2010, 10, 3739-3758.

(29) Li, X.; Ye, J. Comparison of the Electrochemical Reactivity of Carbon Nanotubes Paste Electrodes with Different Types of Multiwalled Carbon Nanotubes. Electroanalysis 2008, 20, 1917-1924.

(30) Zhang, X.; Jiao, K.; Wang, X. Paste Electrode Based on Short Single-Walled Carbon Nanotubes and Room Temperature Ionic Liquid: Preparation, Characterization and Application in DNA Detection. Electroanalysis 2008, 20, 1361-1366.

(31) Xiao, F.; Ruan, C.; Liu, L.; Yan, R.; Zhao, F.; Zeng, B. Single-Walled Carbon Nanotube-lonic Liquid Paste Electrode for the Sensitive Voltammetric Determination of Folic Acid. Sensors Actuators, B Chem. 2008, 134, 895-901.

(32) Valentini, F.; Amine, A.; Orlanducci, S.; Terranova, M. L.; Palleschi, G. Carbon Nanotube Purification: Preparation and Characterization of Carbon Nanotube Paste Electrodes. Anal. Chem. 2003, 75, 5413-5421. 
(33) Rubianes, M. D.; Rivas, G. A. Carbon Nanotubes Paste Electrode. Electrochem. commun. 2003, 5, 689-694.

(34) Jang, E. Y.; Kang, T. J.; Im, H. W.; Kim, D. W.; Kim, Y. H. Single-Walled CarbonNanotube Networks on Large-Area Glass Substrate by the Dip-Coating Method. Small 2008, 4, 2235-2261.

(35) Mirri, F.; Ma, A. W. K.; Hsu, T. T.; Behabtu, N.; Eichmann, S. L.; Young, C. C.; Tsentalovich, D. E.; Pasquali, M. High-Performance Carbon Nanotube Transparent Conductive Films by Scalable Dip Coating. ACS Nano 2012, 97379744.

(36) Yang, M.; Kim, D.-H.; Kim, W.-S.; Kang, T. J.; Lee, B. Y.; Hong, S.; Kim, Y. H.; Hong, S.-H. H(2) Sensing Characteristics of $\mathrm{SnO}(2)$ Coated Single Wall Carbon Nanotube Network Sensors. Nanotechnology 2010, 21, 215501-215508.

(37) Jacobs, C. B.; Vickrey, T. L.; Venton, B. J. Functional Groups Modulate the Sensitivity and Electron Transfer Kinetics of Neurochemicals at Carbon Nanotube Modified Microelectrodes. Analyst 2011, 4, 3557-3565.

(38) Liu, C. Electrochemical Characterization of Films of Single-Walled Carbon Nanotubes and Their Possible Application in Supercapacitors. Electrochem. SolidState Lett. 1999, 2, 577.

(39) Luo, H.; Shi, Z.; Li, N.; Gu, Z.; Zhuang, Q. Investigation of the Electrochemical and Electrocatalytic Behavior of Single-Wall Carbon Nano Tube Film on a Glassy Carbon Electrode. Anal. Chem. 2001, 73, 915-920.

(40) Wang, J.; Li, M.; Shi, Z.; Li, N.; Gu, Z. Electrochemistry of DNA at Single-Wall Carbon Nanotubes. Electroanalysis 2004, 16, 140-144.

(41) Lee, S. W.; Kim, B.-S. S.; Chen, S.; Shao-Horn, Y.; Hammond, P. T. Layer-byLayer Assembly of All Carbon Nanotube Ultrathin Films for Electrochemical Applications. J. Am. Chem. Soc. 2008, 131, 671-679.

(42) Xiao, F.; Ruan, C.; Li, J.; Liu, L.; Zhao, F.; Zeng, B. Voltammetric Determination of Xanthine with a Single-Walled Carbon Nanotube-Ionic Liquid Paste Modified Glassy Carbon Electrode. Electroanalysis 2008, 20, 361-366. 
(43) Zhang, Y.; Shen, Y.; Li, J.; Niu, L.; Dong, S.; Ivaska, A. Electrochemical Functionalization of Single-Walled Carbon Nanotubes in Large Quantities at a Room-Temperature lonic Liquid Supported Three-Dimensional Network Electrode. Langmuir 2005, 21, 4797-4800.

(44) Fan, S.; Xiao, F.; Liu, L.; Zhao, F.; Zeng, B. Sensitive Voltammetric Response of Methylparathion on Single-Walled Carbon Nanotube Paste Coated Electrodes Using lonic Liquid as Binder. Sensors Actuators, B Chem. 2008, 132, 34-39.

(45) Goyal, R. N.; Bishnoi, S. Sensitive Voltammetric Sensor for the Determination of Oxidative DNA Damage in Calf Thymus DNA. Biosens. Bioelectron. 2010, 26, 463-469.

(46) Goyal, R. N.; Chatterjee, S.; Rana, A. R. S.; Chasta, H. Application of Modified Pyrolytic Graphite Electrode as a Sensor in the Simultaneous Assay of Adenine and Adenosine Monophosphate. Sensors Actuators, B Chem. 2011, 156, 198203.

(47) Kim, S. Y.; Kim, Y.; Lee, K. M.; Yoon, W. S.; Lee, H. S.; Lee, J. T.; Kim, S.; Ahn, Y. H.; Park, J.; Lee, T. K.; et al. Fully Solution-Processed Transparent Conducting Oxide-Free Counter Electrodes for Dye-Sensitized Solar Cells: Spray-Coated Single-Wall Carbon Nanotube Thin Films Loaded with Chemically-Reduced Platinum Nanoparticles. ACS Appl. Mater. Interfaces 2014, 6, 13430-13437.

(48) Kim, J. H.; Nam, K. W.; Ma, S. B.; Kim, K. B. Fabrication and Electrochemical Properties of Carbon Nanotube Film Electrodes. Carbon N. Y. 2006, 44, 19631968.

(49) Bekyarova, E.; Itkis, M. E.; Cabrera, N.; Zhao, B.; Yu, A.; Gao, J.; Haddon, R. C. Electronic Properties of Single-Walled Carbon Nanotube Networks. J. Am. Chem. Soc. 2005, 127, 5990-5995.

(50) Kaiser, A. B.; Skaalova, V.; Roth, S. Modelling Conduction in Carbon Nanotube Networks with Different Thickness, Chemical Treatment and Irradiation. Phys. E 2008, 40, 2311-2318.

(51) Martinez, A. W.; Phillips, S. T.; Whitesides, G. M.; Carrilho, E. Diagnostics for the Developing World: Microfluidic Paper-Based Analytical Devices. Anal. Chem. 2010, 82, 3-10.

(52) Liang, P.; Yu, H.; Guntupalli, B.; Xiao, Y. Paper-Based Device for Rapid Visualization of NADH Based on Dissolution of Gold Nanoparticles. ACS Appl. Mater. Interfaces 2015, 7, 15023-15030. 
(53) Ge, S.; Ge, L.; Yan, M.; Song, X.; Yu, J.; Huang, J. A Disposable Paper-Based Electrochemical Sensor with an Addressable Electrode Array for Cancer Screening. Chem. Commun. 2012, 48, 9397-9399.

(54) Desmet, C.; Marquette, C. A.; Blum, L. J.; Doumèche, B. Paper Electrodes for Bioelectrochemistry: Biosensors and Biofuel Cells. Biosens. Bioelectron. 2016, $76,145-163$.

(55) Hu, C.; Ding, Y.; Ji, Y.; Xu, J.; Hu, S. Fabrication of Thin-Film Electrochemical Sensors from Single-Walled Carbon Nanotubes by Vacuum Filtration. Carbon N. Y. 2010, 48, 1345-1352.

(56) Da Costa, T. H.; Song, E.; Tortorich, R. P.; Choi, J.-W. A Paper-Based Electrochemical Sensor Using Inkjet-Printed Carbon Nanotube Electrodes. ECS J. Solid State Sci. Technol. 2015, 4, S3044-S3047.

(57) Guntupalli, B.; Liang, P.; Lee, J. H.; Yang, Y.; Yu, H.; Canoura, J.; He, J.; Li, W.; Weizmann, Y.; Xiao, Y. Ambient Filtration Method to Rapidly Prepare Highly Conductive, Paper-Based Porous Gold Films for Electrochemical Biosensing. ACS Appl. Mater. Interfaces 2015, 7, 27049-27058.

(58) Lodish, H.; Berk, A.; Zipursky, S. L.; Matsudaira, P.; Baltimore, D.; Darnell, J. Structure of Nucleic Acids. In Molecular Cell Biology 4th edition; New York: W. H. Freeman, 2000.

(59) Pogson, C. I. Guanine Nucleotides and Their Significance in Biochemical Processes. Am. J. Clin. Nutr. 1974, 27, 380-402.

(60) Graff, S.; Engelman, M.; Graff, M. A. Guanine in Cancer. Cancer Res. 1951, 11, 388-392.

(61) Adebambo, K. F.; Folorunsho, K. Synthesis and Biological Evaluation of Novel Guanine-Containing Compounds as Potential HIV-1 Non-Nucleoside Reverse Transcriptase or Integrase Inhibitors. 2010.

(62) Wang, W.; Zhou, L.; Wang, S.; Luo, Z.; Hu, Z. Rapid and Simple Determination of Adenine and Guanine in DNA Extract by Micellar Electrokinetic Chromatography with Indirect Laser-Induced Fluorescence Detection. Talanta 2008, 74, 10501055. 
(63) Wang, J.; Chen, G.; Muck, A.; Shin, D.; Fujishima, A. Microchip Capillary Electrophoresis with a Boron-Doped Diamond Electrode for Rapid Separation and Detection of Purines. J. Chromatogr. A 2004, 1022, 207-212.

(64) Kato, D.; Sekioka, N.; Ueda, A.; Kurita, R.; Hirono, S.; Suzuki, K.; Niwa, O. A Nanocarbon Film Electrode as a Platform for Exploring DNA Methylation. J. Am. Chem. Soc. 2008, 130, 3716-3717.

(65) Cesar Prado; Flechsig, G.-U.; Peter Grundler; John S. Frood; Frank Marken; Richard G. Compton. Electrochemical Analysis of Nucleic Acids at Boron-Doped Diamond Electrodes. Analyst 2002, 127, 329-332.

(66) Wang, H.; Ju, H.; Chen, H. Simultaneous Determination of Guanine and Adenine in an Electrochemically Pretreated Glassy Carbon Electrode. Anal. Chim. Acta 2012, 461, 243-250.

(67) Yin, H.; Zhou, Y.; Ma, Q.; Ai, S.; Ju, P.; Zhu, L.; Lu, L. Electrochemical Oxidation Behavior of Guanine and Adenine on graphene-Nafion Composite Film Modified Glassy Carbon Electrode and the Simultaneous Determination. Process Biochem. 2010, 45, 1707-1712.

(68) Zhou, M.; Zhai, Y.; Dong, S. Electrochemical Sensing and Biosensing Platform Based on Chemically Reduced Graphene Oxide. Anal. Chem. 2009, 81, 56035613.

(69) Ahammad, S. A. J.; Lee, J.-J.; Rahman, M. A. Electrochemical Sensors Based on Carbon Nanotubes. Sensors 2009, 9, 2289-2319.

(70) Kumar, S. A.; Chen, S.-M. Electroanalysis of NADH Using Conducting and Redox Active Polymer/Carbon Nanotubes Modified Electrodes-A Review. Sensors 2008, 8, 739-766.

(71) Wang, J.; Chen, G.; Chatrathi, M. P.; Musameh, M. Capillary Electrophoresis Microchip with a Carbon Nanotube-Modified Electrochemical Detector. Anal. Chem. 2004, 76, 298-302.

(72) Mazloum-Ardakani, M.; Beitollahi, H.; Ganjipour, B.; Naeimi, H.; Nejati, M. Electrochemical and Catalytic Investigations of Dopamine and Uric Acid by Modified Carbon Nanotube Paste Electrode. Bioelectrochemistry 2009, 75, 1-8. 
(73) Balan, I.; David, I. G.; David, V.; Stoica, A. I.; Mihailciuc, C.; Stamatin, I.; Ciucu, A. A. Electrocatalytic Voltammetric Determination of Guanine at a Cobalt Phthalocyanine Modified Carbon Nanotubes Paste Electrode. J. Electroanal. Chem. 2011, 654, 8-12.

(74) Erdem, A.; Papakonstantinou, P.; Murphy, H. Direct DNA Hybridization at Disposable Graphite Electrodes Modified with Carbon Nanotubes. Anal. Chem. 2006, 78, 6656-6659.

(75) Wang, J.; Kawde, A.-N.; Musameh, M. Carbon-Nanotube-Modified Glassy Carbon Electrodes for Amplified Label-Free Electrochemical Detection of DNA Hybridization. Analyst 2003, 128, 912-916.

(76) Abbaspour, A.; Noori, A. A Cyclodextrin Host-guest Recognition Approach to a Label-Free Electrochemical DNA Hybridization Biosensor. Analyst 2012, 137, 1860.

(77) Erdem, A.; Karadeniz, H.; Caliskan, A. Single-Walled Carbon Nanotubes Modified Graphite Electrodes for Electrochemical Monitoring of Nucleic Acids and Biomolecular Interactions. Electroanalysis 2009, 21, 464-471.

(78) Deng, C.; Xia, Y.; Xiao, C.; Nie, Z.; Yang, M.; Si, S. Electrochemical Oxidation of Purine and Pyrimidine Bases Based on the Boron-Doped Nanotubes Modified Electrode. Biosens. Bioelectron. 2012, 31, 469-474.

(79) Wu, K.; Fei, J.; Bai, W.; Hu, S. Direct Electrochemistry of DNA, Guanine and Adenine at a Nanostructured Film-Modified Electrode. Anal. Bioanal. Chem. 2003, 376, 205-209.

(80) Karadeniz, H.; Erdem, A.; Caliskan, A. Electrochemical Monitoring of DNA Hybridization by Multiwalled Carbon Nanotube Based Screen Printed Electrodes. Electroanalysis 2008, 20, 1932-1938.

(81) Ye, Y.; Ju, H. Rapid Detection of ssDNA and RNA Using Multi-Walled Carbon Nanotubes Modified Screen-Printed Carbon Electrode. Biosens. Bioelectron. 2005, 21, 735-741.

(82) Xiao, F.; Zhao, F.; Li, J.; Liu, L.; Zeng, B. Characterization of Hydrophobic Ionic Liquid-Carbon Nanotubes-Gold Nanoparticles Composite Film Coated Electrode and the Simultaneous Voltammetric Determination of Guanine and Adenine. Electrochim. Acta 2008, 53, 7781-7788. 
(83) Wang, P.; Wu, H.; Dai, Z.; Zou, X. Simultaneous Detection of Guanine, Adenine, Thymine and Cytosine at Choline Monolayer Supported Multiwalled Carbon Nanotubes Film. Biosens. Bioelectron. 2011, 26, 3339-3345.

(84) Liu, T.; Zhu, X.; Cui, L.; Ju, P.; Qu, X.; Ai, S. Simultaneous Determination of Adenine and Guanine Utilizing $\mathrm{PbO}_{2}$-Carbon Nanotubes-Ionic Liquid Composite Film Modified Glassy Carbon Electrode. J. Electroanal. Chem. 2011, 651, 216221.

(85) Tang, C.; Umasankar, Y.; Chen, S. M. Simultaneous Determination of Adenine Guanine and Thymine at Multi-Walled Carbon Nanotubes Incorporated with Poly(new Fuchsin) Composite Film. Anal. Chim. Acta 2009, 636, 19-27.

(86) Shen, Q.; Wang, X. Simultaneous Determination of Adenine, Guanine and Thymine Based on $\beta$-cyclodextrin/MWNTs Modified Electrode. J. Electroanal. Chem. 2009, 632, 149-153.

(87) Umasankar, Y.; Thiagarajan, S.; Chen, S. M. Pinecone Shape Hydroxypropyl- $\beta$ Cyclodextrin on a Film of Multi-Walled Carbon Nanotubes Coated with Gold Particles for the Simultaneous Determination of Tyrosine, Guanine, Adenine and Thymine. Carbon N. Y. 2007, 45, 2783-2796.

(88) Liu, H.; Wang, G.; Chen, D.; Zhang, W.; Li, C.; Fang, B. Fabrication of polythionine/NPAu/MWNTs Modified Electrode for Simultaneous Determination of Adenine and Guanine in DNA. Sensors Actuators B Chem. 2008, 128, 414-421.

(89) Cai, H.; Cao, X.; Jiang, Y.; He, P.; Fang, Y. Carbon Nanotube-Enhanced Electrochemical DNA Biosensor for DNA Hybridization Detection. Anal. Bioanal. Chem. 2003, 375, 287-293.

(90) Bollo, S.; Ferreyra, N. F.; Rivas, G. A. Electrooxidation of DNA at Glassy Carbon Electrodes Modified with Multiwall Carbon Nanotubes Dispersed in Chitosan. Electroanalysis 2007, 19, 833-840.

(91) Zhang, S.; Ji, C.; Bian, Z.; Yu, P.; Zhang, L.; Liu, D.; Shi, E.; Shang, Y.; Peng, H.; Cheng, Q.; et al. Porous, Platinum Nanoparticle-Adsorbed Carbon Nanotube Yarns for Efficient Fiber Solar Cells. ACS Nano 2012, 6, 7191-7198.

(92) Hrapovic, S.; Liu, Y.; Male, K. B.; Luong, J. H. T. Electrochemical Biosensing Platforms Using Platinum Nanoparticles and Carbon Nanotubes. Anal. Chem. 2004, 76, 1083-1088. 
(93) Zhang, S.; Yuan, X.-Z.; Hin, J. N. C.; Wang, H.; Friedrich, K. A.; Schulze, M. A Review of Platinum-Based Catalyst Layer Degradation in Proton Exchange Membrane Fuel Cells. J. Power Sources 2009, 194, 588-600.

(94) Yu, W.; Porosoff, M. D.; Chen, J. G. Review of Pt-Based Bimetallic Catalysis: From Model Surfaces to Supported Catalysts. Chem. Rev. 2012, 112, 5780-5817.

(95) Tian, Z. Q.; Jiang, S. P.; Liang, Y. M.; Shen, P. K. Synthesis and Characterization of Platinum Catalysts on Muldwalled Carbon Nanotubes by Intermittent Microwave Irradiation for Fuel Cell Applications. J. Phys. Chem. B 2006, 110, 5343-5350.

(96) Wang, H. Y.; Wang, F. M.; Wang, Y. Y.; Wan, C. C.; Hwang, B. J.; Santhanam, R.; Rick, J. Electrochemical Formation of Pt Nanoparticles on Multiwalled Carbon Nanotubes: Useful for Fabricating Electrodes for Use in Dye-Sensitized Solar Cells. J. Phys. Chem. C 2011, 115, 8439-8446.

(97) Planeix, J. M.; Coustel, N.; Coq, B.; Brotons, V.; Kumbhar, P. S.; Dutartre, R.; Geneste, P.; Bernier, P.; Ajayan, P. M. Application of Carbon Nanotubes as Supports in Heterogeneous Catalysis. J. Am. Chem. Soc. 1994, 116, 7935-7936.

(98) Wu, B.; Hu, D.; Kuang, Y.; Liu, B.; Zhang, X.; Chen, J. Functionalization of Carbon Nanotubes by an Ionic-Liquid Polymer: Dispersion of Pt and PtRu Nanoparticles on Carbon Nanotubes and Their Electrocatalytic Oxidation of Methanol. Angew. Chemie - Int. Ed. 2009, 48, 4751-4754.

(99) Liu, R.; Ding, H.; Lin, J.; Shen, F.; Cui, Z.; Zhang, T. Fabrication of PlatinumDecorated Single-Walled Carbon Nanotube Based Hydrogen Sensors by Aerosol Jet Printing. Nanotechnology 2012, 23, 505301.

(100) Wang, D.; Li, Z.; Chen, L. Templated Synthesis of Single-Walled Carbon Nanotube and Metal Nanoparticle Assemblies in Solution. JACS Commun. 2006, 128, 15078-15079.

(101) Hsin, Y. L.; Hwang, K. C.; Yeh, C.-T. Poly(vinylpyrrolidone)-Modified Graphite Carbon Nanofibers as Promising Supports for PtRu Catalysts in Direct Methanol Fuel Cells. J. Am. Chem. Soc. 2007, 129, 9999-10010.

(102) Wang, S.; Wang, X.; Jiang, S. P. PtRu Nanoparticles Supported on 1Aminopyrene-Functionalized Multiwalled Carbon Nanotubes and Their Electrocatalytic Activity for Methanol Oxidation. Langmuir 2008, 24, 10505-10512. 
(103) Wu, B.; Hu, D.; Kuang, Y.; Yu, Y.; Zhang, X.; Chen, J. High Dispersion of Platinum-Ruthenium Nanoparticles on the 3,4,9,10-Perylene Tetracarboxylic AcidFunctionalized Carbon Nanotubes for Methanol Electro-Oxidation. Chem. Commun. 2011, 47, 5253-5255.

(104) Selvaraj, V.; Grace, A. N.; Alagar, M. Electrocatalytic Oxidation of Formic Acid and Formaldehyde on Nanoparticle Decorated Single Walled Carbon Nanotubes. J. Colloid Interface Sci. 2009, 333, 254-262.

(105) Lin, Z.; Chu, H.; Shen, Y.; Wei, L.; Liu, H.; Li, Y. Rational Preparation of Faceted Platinum Nanocrystals Supported on Carbon Nanotubes with Remarkably Enhanced Catalytic Performance. Chem. Commun. 2009, 7167-7169.

(106) Liao, S.; Holmes, K.-A.; Tsaprailis, H.; Birss, V. I. High Performance PtRulr Catalysts Supported on Carbon Nanotubes for the Anodic Oxidation of Methanol. J. Am. Chem. Soc. 2006, 128, 3504-3505.

(107) Li, L.; Xing, Y. Pt-Ru Nanoparticles Supported on Carbon Nanotubes as Methanol Fuel Cell Catalysts. J. Phys. Chem. C 2007, 111, 2803-2808.

(108) Liu, Z.; Ling, X. Y.; Guo, B.; Hong, L.; Lee, J. Y. Pt and PtRu Nanoparticles Deposited on Single-Wall Carbon Nanotubes for Methanol Electro-Oxidation. J. Power Sources 2007, 167, 272-280.

(109) Huang, J. E.; Guo, D. J.; Yao, Y. G.; Li, H. L. High Dispersion and Electrocatalytic Properties of Platinum Nanoparticles on Surface-Oxidized Single-Walled Carbon Nanotubes. J. Electroanal. Chem. 2005, 577, 93-97.

(110) Vincenzo Lordi, Nan Yao, and J. W. Method for Supporting Platinum on SingleWalled Carbon Nanotubes for a Selective Hydrogenation Catalyst. Chem. Mater. 2001, 13, 733-737.

(111) Liu, J.; Liu, C.; Wang, F.; Song, Y.; Li, Z.; Ji, J. Preparation of Pt Nanocrystals on Ultrasonic Cavitation Functionalized Pristine Carbon Nanotubes as Electrocatalysts for Electrooxidation of Methanol. Ind. Eng. Chem. Res. 2014, 53, 20099-20106.

(112) Sanles-Sobrido, M.; Correa-Duarte, A.; Carregal-Romero, S.; RodríguezGonzález, B.; Álvarez-Puebla, R. a.; Hervés, P.; Liz-Marzán, L. M. Highly Catalytic Single-Crystal Dendritic Pt Nanostructures Supported on Carbon Nanotubes. Chem. Mater. 2009, 21, 1531-1535. 
(113) Mu, Y.; Liang, H.; Hu, J.; Jiang, L.; Wan, L. Controllable Pt Nanoparticle Deposition on Carbon Nanotubes as an Anode Catalyst for Direct Methanol Fuel Cells. J. Phys. Chem. B 2005, 109, 22212-22216.

(114) Kongkanand, A.; Vinodgopal, K.; Kuwabata, S.; Kamat, P. V. Highly Dispersed Pt Catalysts on Single-Walled Carbon Nanotubes and Their Role in Methanol Oxidation. J. Phys. Chem. B Lett. 2006, 110, 16185-16188.

(115) Harriman, A.; Millward, G. R.; Neta, P.; Richoux, M. C.; Thomas, J. M. Interfacial Electron-Transfer Reactions between Platinum Colloids and Reducing Radicals in Aqueous Solution. J. Phys. Chem. 1988, 92, 1286-1290.

(116) Dalmia, A.; Lineken, C. L.; Savinell, R. F. Synthesis of Ion Conducting Polymer Protected Nanometer Size Platinum Colloids. J. Colloid Interface Sci. 1998, 205, 535-537.

(117) Morsbach, E.; Speder, J.; Arenz, M.; Brauns, E.; Lang, W.; KUnz, S.; Bäumer, M. Stabilizing Catalytically Active Nanoparticles by Ligand-Linking: Towards Three Dimensional Networks with High Catalytic Surface Area. Langmuir 2014, 30 , 5564-5573.

(118) Schrader, I.; Warneke, J.; Backenkohler, J.; Kunz, S. Functionalization of Platinum Nanoparticles with L-Proline: Simultaneous Enhancements of Catalytic Activity and Selectivity. J. Am. Chem. Soc. 2015, 137, 905-912.

(119) Chen, J.; Herricks, T.; Geissler, M.; Xia, Y. Single-Crystal Nanowires of Platinum Can Be Synthesized by Controlling the Reaction Rate of a Polyol Process. J. Am. Chem. Soc. 2004, 126, 10854-10855.

(120) Zhang, S.; Ji, C.; Bian, Z.; Yu, P.; Zhang, L.; Liu, D.; Shi, E.; Shang, Y.; Peng, H.; Cheng, Q.; et al. Porous, Platinum Nanoparticle-Adsorbed Carbon Nanotube Yarns for Efficient. ACS Nano 2012, 7191-7198.

(121) Quinn, B. M.; Dekker, C.; Lemay, S. G. Electrodeposition of Noble Metal Nanoparticles on Carbon Nanotubes. J. Am. Chem. Soc. 2005, 127, 6146-6147.

(122) Zhao, Y.; Fan, L.; Zhong, H.; Li, Y.; Yang, S. Platinum Nanoparticle Clusters Immobilized on Multiwalled Carbon Nanotubes: Electrodeposition and Enhanced Electrocatalytic Activity for Methanol Oxidation. Adv. Funct. Mater. 2007, 17, 1537-1541. 
(123) Wu, G.; Chen, Y. S.; Xu, B. Q. Remarkable Support Effect of SWNTs in Pt Catalyst for Methanol Electrooxidation. Electrochem. commun. 2005, 7, 12371243.

(124) Day, T. M.; Unwin, P. R.; Wilson, N. R.; Macpherson, J. V. Electrochemical Templating of Metal Nanoparticles and Nanowires on Single-Walled Carbon Nanotube Networks. J. Am. Chem. Soc. 2005, 127, 10639-10647.

(125) Choi, H. C.; Shim, M.; Bangsaruntip, S.; Dai, H. Spontaneous Reduction of Metal Ions on the Sidewalls of Carbon Nanotubes. J. Am. Chem. Soc. 2002, 124, 90589059 .

(126) Qu, L.; Dai, L. Substrate-Enhanced Electroless Deposition of Metal Nanoparticles on Carbon Nanotubes. J. Am. Chem. Soc. 2005, 10806-10807.

(127) Tsai, M. C.; Yeh, T. K.; Tsai, C. H. Methanol Oxidation Efficiencies on CarbonNanotube-Supported Platinum and Platinum-Ruthenium Nanoparticles Prepared by Pulsed Electrodeposition. Int. J. Hydrogen Energy 2011, 36, 8261-8266.

(128) Tsai, M. C.; Yeh, T. K.; Tsai, C. H. An Improved Electrodeposition Technique for Preparing Platinum and Platinum-Ruthenium Nanoparticles on Carbon Nanotubes Directly Grown on Carbon Cloth for Methanol Oxidation. Electrochem. commun. 2006, 8, 1445-1452.

(129) Sieben, J. M.; Ansón-Casaos, A.; Martínez, M. T.; Morallón, E. Single-Walled Carbon Nanotube Buckypapers as Electrocatalyst Supports for Methanol Oxidation. J. Power Sources 2013, 242, 7-14.

(130) Zhu, W.; Ku, D.; Zheng, J. P.; Liang, Z.; Wang, B.; Zhang, C.; Walsh, S.; Au, G.; Plichta, E. J. Buckypaper-Based Catalytic Electrodes for Improving Platinum Utilization and PEMFC's Performance. Electrochim. Acta 2010, 55, 2555-2560.

(131) Zhu, W.; Zheng, J. P.; Liang, R.; Wang, B.; Zhang, C.; Au, G.; Plichtae, E. J. Durability Study on SWNT / Nanofiber Buckypaper Catalyst Support for PEMFCs. J. Electrochem. Soc. 2009, 156, B1099-B1105.

(132) Claussen, J. C.; Daniele, M. A.; Geder, J.; Pruessner, M.; Ma, A. J.; Melde, B. J.; Twigg, M.; Verbarg, J. M.; Medintz, I. L. Platinum-Paper Micromotors: An Urchinlike Nanohybrid Catalyst for Green Monopropellant Bubble-Thrusters. ACS Appl. Mater. Interfaces Interfaces 2014, 6, 17837-17847. 
(133) Marr, K. M.; Chen, B.; Mootz, E. J.; Geder, J.; Pruessner, M.; Melde, B. J.; Vanfleet, R. R.; Medintz, I. L.; Iverson, B. D.; Claussen, J. C. High Aspect Ratio Carbon Nanotube Membranes Decorated with Pt Nanoparticle Urchins for Micro Underwater Vehicle Propulsion via $\mathrm{H}_{2} \mathrm{O}_{2}$ Decomposition. ACS Nano 2015, 9, 7791-9803.

(134) Yuan, J.; Wang, Z.; Zhang, Y.; Shen, Y.; Han, D.; Zhang, Q.; Xu, X.; Niu, L. Electrostatic Layer-by-Layer of a Platinum-Loaded Multiwall Carbon Nanotube Multilayer: A Tunable Catalyst Film for Anodic Methanol Oxidation. Thin Solid Films 2008, 516, 6531-6535.

(135) Bergamaski, K.; Pinheiro, A. L. N.; Teixeira-Neto, E.; Nart, F. C. Nanoparticle Size Effects on Methanol Electrochemical Oxidation on Carbon Supported Platinum Catalysts. J. Phys. Chem. B 2006, 110, 19271-19279.

(136) Ma, L.; Wang, C.; Gong, M.; Liao, L.; Long, R.; Wang, J.; Wu, D.; Zhong, W.; Kim, M. J.; Chen, Y.; et al. Control over the Branched Structures of Platinum Nanocrystals for Electrocatalytic Applications. ACS Nano 2012, 6, 9797-9806.

(137) Hansan, L.; Jiujun, Z. Electrocatalysis of Direct Methanol Fuel Cells: From Fundamentals to Applications; 15th ed.; Weinheim: Wiley-VCH, 2009.

(138) Nassr, A. B. A. A.; Sinev, I.; Pohl, M. M.; Grünert, W.; Bron, M. Rapid MicrowaveAssisted Polyol Reduction for the Preparation of Highly Active PtNi/CNT Electrocatalysts for Methanol Oxidation. ACS Catal. 2014, 4, 2449-2462.

(139) Hamnett, A. Mechanism and Electrocatalysis in the Direct Methanol Fuel Cell. Catal. Today 1997, 38, 445-457.

(140) Turkevich, J.; Stevenson, P. C.; Hillier, J. A Study of the Nucleation and Growth Processes in the Synthesis of Colloidal Gold. Discuss. Faraday Soc. 1951, 11, 55-75.

(141) Kimling, J.; Maier, M.; Okenve, B.; Kotaidis, V.; Ballot, H.; Plech, A. Turkevich Method for Gold Nanoparticle Synthesis Revisited. J. Phys. Chem. B 2006, 110, 15700-15707.

(142) Brust, M.; Walker, M.; Bethell, D.; Schiffrin, D. J.; Whyman, R.; Thomas, J. M.; Edwards, P. P.; Faraday, M.; Porter, M. D.; Bright, T. B.; et al. Synthesis of ThiolDerivatised Gold Nanoparticles in a Two-Phase Liquid-Liquid System. J. Chem. Soc., Chem. Commun. 1994, 0, 801-802. 
(143) Willets, K. A.; Van Duyne, R. P. Localized Surface Plasmon Resonance Spectroscopy and Sensing. Annu. Rev. Phys. Chem 2007, 58, 267-297.

(144) Kelly, K. L.; Coronado, E.; Zhao, L. L.; Schatz, G. C. The Optical Properties of Metal Nanoparticles: The Influence of Size, Shape, and Dielectric Environment. J. Phys. Chem. B 2003, 107, 668-677.

(145) Mayer, K. M.; Hafner, J. H. Localized Surface Plasmon Resonance Sensors. Chem. Rev. 2011, 111, 3828-3857.

(146) Liz-Marzán, L. M. Tailoring Surface Plasmons through the Morphology and Assembly of Metal Nanoparticles. Langmuir 2006, 22, 32-41.

(147) Liu, X.; Atwater, M.; Wang, J.; Huo, Q. Extinction Coefficient of Gold Nanoparticles with Different Sizes and Different Capping Ligands. Colloids Surfaces B Biointerfaces 2007, 58, 3-7.

(148) Hao, E.; Bailey, R. C.; Schatz, G. C.; Hupp, J. T.; Li, S. Synthesis and Optical Properties of "Branched" Gold Nanocrystals. Nano Lett. 2004, 4, 327-330.

(149) Nehl, C. L.; Liao, H.; Hafner, J. H. Optical Properties of Star-Shaped Gold Nanoparticles. Nano Lett. 2006, 6, 683-688.

(150) Sener, G.; Uzun, L.; Denizli, A. Lysine-Promoted Colorimetric Response of Gold Nanoparticles: A Simple Assay for Ultrasensitive Mercury(II) Detection. Anal. Chem. 2014, 86, 514-520.

(151) Hurst, S. J.; Lytton-Jean, A. K. R.; Mirkin, C. A. Maximizing DNA Loading on a Range of Gold Nanoparticle Sizes. Anal. Chem. 2006, 78, 8313-8318.

(152) Majzik, A.; Fülöp, L.; Csapó, E.; Bogár, F.; Martinek, T.; Penke, B.; Bíró, G.; Dékány, I. Functionalization of Gold Nanoparticles with Amino Acid, $\beta$-Amyloid Peptides and Fragment. Colloids Surfaces B Biointerfaces 2010, 81, 235-241.

(153) Jazayeri, M. H.; Amani, H.; Pourfatollah, A. A.; Pazoki-Toroudi, H.; Sedighimoghaddam, B. Various Methods of Gold Nanoparticles (GNPs) Conjugation to Antibodies. Sens. Bio-Sensing Res. 2016, 9, 17-22.

(154) Zhao, W.; Chiuman, W.; Brook, M. A.; Li, Y. Simple and Rapid Colorimetric Biosensors Based on DNA Aptamer and Noncrosslinking Gold Nanoparticle Aggregation. Chem. Commun. 2007, 8, 727-731. 
(155) Xu, X.; Han, M. S.; Mirkin, C. A. A Gold-Nanoparticle-Based Real-Time Colorimetric Screening Method for Endonuclease Activity and Inhibition. Angew. Chemie 2007, 119, 3538-3540.

(156) Song, G.; Chen, C.; Ren, J.; Qu, X. A Simple, Universal Colorimetric Assay for Endonuclease / Methyltransferase Activity and Inhibition Basd on an EnZymeResponsive Nanoparticle System. ACS Nano 2009, 3, 1183-1189.

(157) Wang, Z.; Lévy, R.; Fernig, D. G.; Brust, M. Kinase-Catalyzed Modification of Gold Nanoparticles: A New Approach to Colorimetric Kinase Activity Screening. J. Am. Chem. Soc. 2006, 128, 2214-2215.

(158) Choi, Y.; Ho, N.-H.; Tung, C.-H. Sensing Phosphatase Activity by Using Gold Nanoparticles. Angew. Chemie - Int. Ed. 2006, 46, 707-709.

(159) Elghanian, R.; Storhoff, J. J.; Mucic, R. C.; Letsinger, R. L.; Mirkin, C. A. Selective Colorimetric Detection of Polynucleotides Based on the Distance-Dependent Optical Properties of Gold Nanoparticles. Science 1997, 277, 1078-1080.

(160) Liu, J.; Lu, Y. A Colorimetric Lead Biosensor Using DNAzyme-Directed Assembly of Gold Nanoparticles. J. Am. Chem. Soc. 2003, 125, 6642-6643.

(161) Zhao, W.; Lam, J. C. F.; Chiuman, W.; Brook, M. A.; Li, Y. Enzymatic Cleavage of Nucleic Acids on Gold Nanoparticles: A Generic Platform for Facile Colorimetric Biosensors. Small 2008, 4, 810-816.

(162) Zhao, W.; Chiuman, W.; Brook, M. A.; Li, Y. Simple and Rapid Colorimetric Biosensors Based on DNA Aptamer and Noncrosslinking Gold Nanoparticle Aggregation. ChemBioChem 2007, 8, 727-731.

(163) Wu, Z. K. Z.; Wu, Z. K. Z.; Tang, H.; Tang, L. J.; Jiang, J. H. Activity-Based DNAGold Nanoparticle Probe as Colorimetric Biosensor for DNA Methyltransferase/glycosylase Assay. Anal. Chem. 2013, 85, 4376-4383.

(164) Ou, L.-J.; Jin, P.-Y.; Chu, X.; Jiang, J.-H.; Yu, R.-Q. Sensitive and Visual Detection of Sequence-Specific DNA-Binding Protein via a Gold NanoparticleBased Colorimetric Biosensor. Anal. Chem. 2010, 82, 6015-6024.

(165) Wu, S.; Liang, P.; Yu, H.; Xu, X.; Liu, Y.; Lou, X.; Xiao, Y. Amplified Single BasePair Mismatch Detection via Aggregation of Exonuclease-Sheared Gold Nanoparticles. Anal. Chem. 2014, 86, 3461-3467. 
(166) Zhang, X.; Servos, M. R.; Liu, J. Surface Science of DNA Adsorption onto CitrateCapped Gold Nanoparticles. Langmuir 2012, 28, 3896-3902.

(167) Parak, W. J.; Pellegrino, T.; Micheel, C. M.; Gerion, D.; Williams, S. C.; Alivisatos, A. P. Conformation of Oligonucleotides Attached to Gold Nanocrystals Probed by Gel Electrophoresis. Nano Lett. 2003, 3, 33-36.

(168) Park, S.; Brown, K. A.; Hamad-Schifferli, K. Changes in Oligonucleotide Conformation on Nanoparticle Surfaces by Modification with Mercaptohexanol. Nano Lett. 2004, 4, 1925-1929.

(169) Chen, C.; Wang, W.; Ge, J.; Zhao, X. S. Kinetics and Thermodynamics of DNA Hybridization on Gold Nanoparticles. Nucleic Acids Res. 2009, 37, 3756-3765.

(170) Peterson, A. W.; Heaton, R. J.; Georgiadis, R. M. The Effect of Surface Probe Density on DNA Hybridization. Nucleic Acids Res. 2001, 29, 5163-5168.

(171) Wang, J.; Zhou, H. S. Aptamer-Based Au Nanoparticles-Enhanced Surface Plasmon Resonance Detection of Small Molecules. Anal. Chem. 2008, 80, 71747178.

(172) Luo, C.; Tang, H.; Cheng, W.; Yan, L.; Zhang, D.; Ju, H. A Sensitive Electrochemical DNA Biosensor for Specific Detection of Enterobacteriaceae Bacteria by Exonuclease III-Assisted Signal Amplification. Biosens. Bioelectron. 2013, 48, 132-137.

(173) Wu, D.; Yin, B.-C.; Ye, B.-C. A Label-Free Electrochemical DNA Sensor Based on Exonuclease III-Aided Target Recycling Strategy for Sequence-Specific Detection of Femtomolar DNA. Biosens. Bioelectron. 2011, 28, 232-238.

(174) Oh, J.-H.; Lee, J.-S. Designed Hybridization Properties of DNA À Gold Nanoparticle. Anal. Chem. 2011, 1, 7364-7370.

(175) Weiss, B. Endonuclease II of Escherichia Coli Is Exonuclease III. J. Biol. Chem. 1976, 251, 1896-1901.

(176) Kow, W. Y. Mechanism of Action of Escherichia Coli Exonuclease III. Biochemistry 1989, 3280-3287. 
(177) Takeuchiso, M.; Lillisn, R.; Demplen, B.; Takeshitasii, M. Interactions of Escherichia Coli Endonuclease IV and Exonuclease III with Abasic Sites in DNA. J. Biol. Chem. 1994, 269, 21907-21914.

(178) Mol, D. C.; Kuo, C.F.; Thayer, M.M; Cunningham P.R.; Tainer A.J. Structure and Function of the Multifunctional DNA-Repair Enzyme Exonuclease III. Nature 1995, $374,381-386$.

(179) Demple, B.; Harrison, L. Repair of Oxidative Damage to DNA: Enzymology and Biology. Annu. Rev. Biochem. 1994, 63, 915-948.

(180) Wilson III, D. M.; Takeshita, M.; Grollman, A. P.; Demple, B. Incision Activity of Human Endonuclease (Ape) at Abasic Site Analogs in DNA. J. Biol. Chem. 1995, 270, 16002-16007.

(181) Roychoudhury, R.; Wu, R. Novel Properties of Escherichia Coli Exonuclease III. J. Biol. Chem. 1977, 252, 4786-4789.

(182) Hoheisel, J. D. On the Activities of Escherichia Coli Exonuclease III. Anal. Biochem. 1993, 209, 238-246.

(183) Cowan, J. A. Metal-Mediated Hydrolysis of Biological Phosphate Esters A Critical Analysis of the Essential Metal Ion Stoichiometry for Magnesium-Dependent Nuclease Activation. J. Biol. Inorg. Chem. 1997, 2, 168-176.

(184) Luo, M.; Kelley, M. R. Inhibition of the Human Apurinic/apyrimidinic Endonuclease (Ape1) Repair Activity and Sensitization of Breast Cancer Cells to DNA Alkylating Agents with Lucanthone. Anticancer Res. 2004, 24, 2127-2134.

(185) Abbotts, R.; Madhusudan, S. Human AP Endonuclease 1 (APE1): From Mechanistic Insights to Druggable Target in Cancer. Cancer Treat. Rev. 2010, 36, 425-435.

(186) Nyland, R. L.; Luo, M.; Kelley, M. R.; Borch, R. F. Design and Synthesis of Novel Quinone Inhibitors Targeted to the Redox Function of Apurinic/apyrimidinic Endonuclease 1/redox Enhancing Factor-1 (Ape1/Ref-1). J. Med. Chem. 2010, 53, 1200-1210.

(187) Shevelev, I. V.; Hübscher, U. The 3'-5' Exonucleases. Nat. Rev. Mol. Cell Biol. 2002, 3, 364-376. 
(188) Belmont, P.; Demeunynck, M.; Constant, J.-F.; Lhomme, J. Synthesis and Study of a New Adenine-acridine Tandem, Inhibitor of Exonuclease III; Bioorg.Med.Chem.Lett. 2000; Vol. 10.

(189) Zuo, X.; Xia, F.; Xiao, Y.; Plaxco, K. Sensitive and Selective Amplified Fluorescence DNA Detection Based on Exonuclease III-Aided Target Recycling. J. Am. Chem. Soc. 2010, 132, 1816-1818.

(190) Liu, S.; Wang, C.; Zhang, C.; Wang, Y.; Tang, B. Label-Free and Ultrasensitive Electrochemical Detection of Nucleic Acids Based on Autocatalytic and Exonuclease III-Assisted Target Recycling Strategy. Anal. Chem. 2013, 85, 22822288.

(191) Chen, H.; Wang, J.; Liang, G.; Zhang, P.; Kong, J. A Novel Exonuclease III Aided Amplification Method for Sensitive Nucleic Acid Detection Based on Single Walled Carbon Nanotube Induced Quenching. Chem. Commun. 2012, 48, 269-271.

(192) Linxweiler, W.; Horz, W. Sequence Specificity of Exonuclease III from E. Coli. Nucleic Acids Res. 1982, 10, 4845-4859.

(193) Takeuchiso, M.; Lillisn, R.; Demplen, B.; Takeshitasii, M. Interactions of Escherichia Coli Endonuclease IV and Exonuclease III with Abasic Sites in DNA. J. Biol. Chem. 1994, 21907-21914.

(194) Wu, X.; Chen, J.; Zhao, J. X. Ultrasensitive Detection of 3'-5' Exonuclease Enzymatic Activity Using Molecular Beacons. Analyst 2014, 139, 1081-1087.

(195) Su, X.; Zhu, X.; Zhang, C.; Xiao, X.; Zhao, M. In Situ, Real-Time Monitoring of the 3 ' to 5' Exonucleases Secreted by Living Cells. Anal. Chem. 2012, 84, 50595065 .

(196) Leung, C.; Chan, D. S.; Man, B. Y.; Wang, C.; Lam, W.; Cheng, Y.; Fong, W.; Hsiao, W. W.; Ma, D. A Simple and Convenient G-Quadruplex-Based Turn-On Fluorescence Assay for $3^{\prime} \rightarrow 5^{\prime}$ Exonuclease Activity. Anal. Chem. 2011, 65, 463-466.

(197) Yang, W.; Ruan, Y.; Wu, W.; Chen, P.; Xu, L.; Fu, F. A "turn-On" and Label-Free Fluorescent Assay for the Rapid Detection of Exonuclease III Activity Based on $\mathrm{Tb}(3+)$-Induced G-Quadruplex Conjugates. Anal. Bioanal. Chem. 2014, 406, $4535-4540$. 
(198) Gooding, J. J. Nanostructuring Electrodes with Carbon Nanotubes: A Review on Electrochemistry and Applications for Sensing. Electrochim. Acta 2005, 50, 30493060 .

(199) Vairavapandian, D.; Vichchulada, P.; Lay, M. D. Preparation and Modification of Carbon Nanotubes: Review of Recent Advances and Applications in Catalysis and Sensing. Anal. Chim. Acta 2008, 626, 119-129.

(200) Trojanowicz, M. Analytical Applications of Carbon Nanotubes: A Review. TrAC Trends Anal. Chem. 2006, 25, 480-489.

(201) Kim, S. N.; Rusling, J. F.; Papadimitrakopoulos, F. Carbon Nanotubes for Electronic and Electrochemical Detection of Biomolecules. Adv. Mater. 2007, 19, 3214-3228.

(202) Harreither, W.; Trouillon, R.; Poulin, P.; Neri, W.; Ewing, A. G.; Safina, G. Carbon Nanotube Fiber Microelectrodes Show a Higher Resistance to Dopamine Fouling. Anal. Chem. 2013, 85, 7447-7453.

(203) Wooten, M.; Gorski, W. Facilitation of NADH Electro-Oxidation at Treated Carbon Nanotubes. Anal. Chem. 2010, 82, 1299-1304.

(204) Yuan, D.; Ding, L.; Chu, H.; Feng, Y.; McNicholas, T. P.; Liu, J. Horizontally Aligned Single-Walled Carbon Nanotube on Quartz from a Large Variety of Metal Catalysts. Nano Lett. 2008, 8, 2576-2579.

(205) Bishnoi, S.; Goyal, R. N.; Shim, Y. B. A Novel Nanogold-Single Wall Carbon Nanotube Modified Sensor for the Electrochemical Determination of 8Hydroxyguanine, a Diabetes Risk Biomarker. Bioelectrochemistry 2014, 99, 2429.

(206) Liana, D. D.; Raguse, B.; Justin Gooding, J.; Chow, E. Recent Advances in Paper-Based Sensors. Sensors 2012, 12, 11505-11526.

(207) Wu, Z.; Chen, Z.; Du, X.; Logan, J. M.; Sippel, J.; Nikolou, M.; Kamaras, K.; Reynolds, J. R.; Tanner, D. B.; Hebard, A. F.; et al. Transparent, Conductive Carbon Nanotube Films. Science 2004, 305, 1273-1276.

(208) De, S.; Lyons, P. E.; Sorel, S.; Doherty, E. M.; King, P. J.; Blau, W. J.; Nirmalraj, P. N.; Boland, J. J.; Scardaci, V.; Joimel, J.; et al. Transparent, Flexible, and Highly Conductive Thin Films Based on Polymer-Nanotube Composites. ACS Nano 2009, 3, 714-720. 
(209) Barisci, J. N.; Wallace, G. G.; Baughman, R. H. Electrochemical Characterization of Single-Walled Carbon Nanotube Electrodes. J. Electrochem. Soc. 2000, 147, 4580 .

(210) Li, J.; Cassell, A.; Delzeit, L.; Han, J.; Meyyappan, M. Novel Three-Dimensional Electrodes: Electrochemical Properties of Carbon Nanotube Ensembles. J. Phys. Chem. B 2002, 106, 9299-9305.

(211) Wilson, N. R.; Guille, M.; Dumitrescu, I.; Fernandez, V. R.; Rudd, N. C.; Williams, C. G.; Unwin, P. R.; Macpherson, J. V. Assessment of the Electrochemical Behavior of Two-Dimensional Networks of Single-Walled Carbon Nanotubes. Anal. Chem. 2006, 78, 7006-7015.

(212) Nicholson, R. S. Theory and Application of Cyclic Voltammetry F M Measurement of Electrode Reaction Kinetics. Anal. Chem. 1965, 37, 1351-1355.

(213) Shukla, M. K.; Dubey, M.; Zakar, E.; Namburu, R.; Czyznikowska, Z.; Leszczynski, J. Interaction of Nucleic Acid Bases with Single-Walled Carbon Nanotube. Chem. Phys. Lett. 2009, 480, 269-272.

(214) Oliveira-Brett, A. M.; Diculescu, V.; Piedade, J. A. . Electrochemical Oxidation Mechanism of Guanine and Adenine Using a Glassy Carbon Microelectrode. Bioelectrochemistry 2002, 55, 61-62.

(215) Akhavan, O.; Ghaderi, E.; Rahighi, R. Toward Single-DNA Electrochemical Biosensing by Graphene Nanowalls. ACS Nano 2012, 6, 2904-2916.

(216) Thangaraj, R.; Senthil Kumar, A. Simultaneous Detection of Guanine and Adenine in DNA and Meat Samples Using Graphitized Mesoporous Carbon Modified Electrode. J. Solid State Electrochem. 2013, 17, 583-590.

(217) Tu, X.; Luo, X.; Luo, S.; Yan, L.; Zhang, F.; Xie, Q. Novel Carboxylation Treatment and Characterization of Multiwalled Carbon Nanotubes for Simultaneous Sensitive Determination of Adenine and Guanine in DNA. Microchim. Acta 2010, 169, 3340.

(218) Wang, Z.; Xiao, S.; Chen, Y. $\beta$-Cyclodextrin Incorporated Carbon NanotubesModified Electrodes for Simultaneous Determination of Adenine and Guanine. J. Electroanal. Chem. 2006, 589, 237-242. 
(219) Yin, H.; Zhou, Y.; Ma, Q.; Liu, T.; Ai, S.; Zhu, L. Electrochemical Oxidation Behavior of Guanosine-5'-Monophosphate on a Glassy Carbon Electrode Modified with a Composite Film of Graphene and Multi-Walled Carbon Nanotubes, and Its Amperometric Determination. Microchim. Acta 2011, 172, 343-349.

(220) Kato, D.; Sumimoto, M.; Ueda, A.; Hirono, S.; Niwa, O. Evaluation of Electrokinetic Parameters for All DNA Bases with Sputter Deposited Nanocarbon Film Electrode. Anal. Chem. 2012, 84, 10607-10613.

(221) Oliveira-Brett, A. M.; Piedade, J. A. P.; Silva, L. A.; Diculescu, V. C. Voltammetric Determination of All DNA Nucleotides. Anal. Biochem. 2004, 332, 321-329.

(222) Goyal, R. N.; Puri, B. K.; Jain, N. Electrochemical Oxidation of Guanosine-5'monophosphate at the Pyrolytic Graphite Electrode. J. Chem. Soc. Perkin Trans. $12001,832-837$.

(223) Chen, A.; Holt-hindle, P. Platinum-Based Nanostructured Materials: Synthesis , Properties, and Applications. Chem. Rev. 2010, 110, 3767-3804.

(224) Hrapovic, S.; Liu, Y.; Male, K. B.; Luong, J. H. T. Electrochemical Biosensing Platforms Using Platinum Nanoparticles and Carbon Nanotubes. Anal. Chem. 2004, 76, 1083-1088.

(225) Wang, C.; Daimon, H.; Onodera, T.; Koda, T.; Sun, S. A General Approach to the Size- and Shape-Controlled Synthesis of Platinum Nanoparticles and Their Catalytic Reduction of Oxygen. Angew. Chemie 2008, 47, 3588-3591.

(226) Pham, T. H.; Le, M. L. P.; Nguyen, T. H.; Nguyen, T. P. T. Synthesis, Properties and Performance of Platinum and Platinum/Carbon Nanotube Films as Cathode Materials for Dye-Sensitized Solar Cells. J. Electrochem. Soc. 2014, 161, 235239.

(227) Quan, X.; Mei, Y.; Xu, H.; Sun, B.; Zhang, X. Optimization of Pt-Pd Alloy Catalyst and Supporting Materials for Oxygen Reduction in Air-Cathode Microbial Fuel Cells. Electrochim. Acta 2015, 165, 72-77.

(228) Kongkanand, A.; Kuwabata, S.; Girishkumar, G.; Kamat, P. Single-Wall Carbon Nanotubes Supported Platinum Nanoparticles with Improved Electrocatalytic Activity for Oxygen Reduction Reaction. Langmuir 2006, 22, 2392-2396. 
(229) Pozio, A.; Francesco, M. De; Cemmi, A.; Cardellini, F.; Giorgi, L. Comparison of High Surface Pt/C Catalysts by Cyclic Voltametry. J. Power Sources 2002, 105, 13-19.

(230) Joo, S. H.; Choi, S. J.; Oh, I.; Kwak, J.; Liu, Z.; Terasaki, O.; Ryoo, R. Ordered Nanoporous Arrays of Carbon Supporting High Dispersions of Platinum Nanoparticles. Nature 2001, 412, 169-172.

(231) Xu, P.; Dong, L.; Neek-Amal, M.; Ackerman, M. L.; Yu, J.; Barber, S. D.; Schoelz, J. K.; Qi, D.; Xu, F.; Thibado, P. M.; et al. Self-Organized Platinum Nanoparticles on Freestanding Graphene. ACS Nano 2014, 8, 2697-2703.

(232) Bigall, N. C.; Härtling, T.; Klose, M.; Simon, P.; Eng, L. M.; Eychmüller, A. Monodisperse Platinum Nanospheres with Adjustable Diameters from 10 to 100 Nm: Synthesis and Distinct Optical Properties. Nano Lett. 2008, 8, 4588-4592.

(233) Marcus, P.; Hinnen, C. XPS Study of the Early Stages of Deposition of Ni, Cu and Pt on HOPG. Surf. Sci. 1997, 392, 134-142.

(234) Borchert, H.; Shevchenko, E. V.; Robert, A.; Mekis, I.; Kornowski, A.; Grübel, G.; Weller, H. Determination of Nanocrystal Sizes: A Comparison of TEM, SAXS, and XRD Studies of Highly Monodisperse $\mathrm{CoPt}_{3}$ Particles. Langmuir 2005, 21, $1931-$ 1936.

(235) Fisica, C.; Milano, U. Real Surface Area Measurements In Electrochemistry. Pure Appl. Chem. 1991, 63, 711-734.

(236) Hyder, N.; Kavian, R.; Sultana, Z.; Saetia, K.; Chen, P.; Lee, S. W.; Shao-horn, Y.; Hammond, P. T. Vacuum-Assisted Layer-by-Layer Nanocomposites for SelfStanding 3D Mesoporous Electrodes. Chem. Mater. 2014, 26, 5310-5318.

(237) Ostojic, G. N.; Liang, Y. T.; Hersam, M. C. Catalytically Active Nanocomposites of Electronically Coupled Carbon Nanotubes and Platinum Nanoparticles Formed via Vacuum Filtration. Nanotechnology 2009, 20, 434019.

(238) Rittié, L.; Perbal, B. Enzymes Used in Molecular Biology: A Useful Guide. J. Cell Commun. Signal. 2008, 2, 25-45.

(239) Byrnes, J. J.; Downey, K. M.; Black, V. L.; So, A. G. A New Mammalian DNA Polymerase with 3' to 5' Exonuclease Activity: DNA Polymerase $\delta$. Biochemistry 1976, 15, 2817-2823. 
(240) Paull, T. T.; Gellert, M. The 3' to 5' Exonuclease Activity of Mre11 Facilitates Repair of DNA Double-Strand Breaks. Mol. Cell 1998, 1, 969-979.

(241) Chaudhry, M. A.; Weinfeld, M. Reactivity of Human Apurinic/apyrimidinic Endonuclease and Escherichia Coli Exonuclease III with Bistranded Abasic Sites in DNA. J. Biol. Chem. 1997, 272, 15650-15655.

(242) Greenberg, M. M.; Weledji, Y. N.; Kim, J.; Bales, B. C. Repair of Oxidized Abasic Sites by Exonuclease III, Endonuclease IV, and Endonuclease III. Biochemistry 2004, 43, 8178-8183.

(243) Sukhanova, M. V.; Khodyreva, S. N.; Lebedeva, N. A.; Prasad, R.; Wilson, S. H.; Lavrik, O. I. Human Base Excision Repair Enzymes Apurinic/apyrimidinic endonuclease1 (APE1), DNA Polymerase $\beta$ And poly(ADP-Ribose) Polymerase 1: Interplay between Strand-Displacement DNA Synthesis and Proofreading Exonuclease Activity. Nucleic Acids Res. 2005, 33, 1222-1229.

(244) Puglisi, F.; Barbone, F.; Tell, G.; Aprile, G.; Pertoldi, B.; Raiti, C.; Kelley, M. R.; Damante, G.; Sobrero, A.; Beltrami, C. A.; et al. Prognostic Role of Ape/Ref-1 Subcellular Expression in Stage I-III Breast Carcinomas. Oncol. Rep. 2002, 9, 1117.

(245) Koukourakis, M. I.; Giatromanolaki, A.; Kakolyris, S.; Sivridis, E.; Georgoulias, V.; Funtzilas, G.; Hickson, I. D.; Gatter, K. C.; Harris, A. L. Nuclear Expression of Human Apurinic/apyrimidinic Endonuclease (HAP1/Ref-1) in Head-and-Neck Cancer Is Associated with Resistance to Chemoradiotherapy and Poor Outcome. Int. J. Radiat. Oncol. Biol. Phys. 2001, 50, 27-36.

(246) Puglisi, F.; Aprile, G.; Minisini, A. M.; Barbone, F.; Cataldi, P.; Tell, G.; Kelley, M. R.; Damante, G.; Beltrami, C. A.; Di Loreto, C. Prognostic Significance of Ape1/ref-1 Subcellular Localization in Non-Small Cell Lung Carcinomas. Anticancer Res. 2001, 21, 4041-4050.

(247) Park, J. S.; Kim, H. L.; Kim, Y. J.; Weon, J. Il; Sung, M. K.; Chung, H. W.; Seo, Y. R. Human AP Endonuclease 1: A Potential Marker for the Prediction of Environmental Carcinogenesis Risk. Oxid. Med. Cell. Longev. 2014, 2014.

(248) Mohammed, M. Z.; Vyjayanti, V. N.; Laughton, C. A.; Dekker, L. V; Fischer, P. M.; Wilson, D. M.; Abbotts, R.; Shah, S.; Patel, P. M.; Hickson, I. D.; et al. Development and Evaluation of Human AP Endonuclease Inhibitors in Melanoma and Glioma Cell Lines. Br. J. Cancer 2011, 104, 653-663. 
(249) Zawahir, Z.; Dayam, R.; Deng, J.; Pereira, C.; Neamati, N. Pharmacophore Guided Discovery of Small-Molecule Human Apurinic/ Apyrimidinic Endonuclease 1 Inhibitors. J. Med. Chem. 2009, 52, 20-32.

(250) Gros, L.; Ishchenko, A. A.; Ide, H.; Elder, R. H.; Saparbaev, M. K. The Major Human AP Endonuclease (Ape1) Is Involved in the Nucleotide Incision Repair Pathway. Nucleic Acids Res. 2004, 32, 73-81.

(251) Daniel, M.-C.; Astruc, D. Gold Nanoparticles: Assembly, Supramolecular Chemistry, Quantum-Size-Related Properties, and Applications toward Biology, Catalysis, and Nanotechnology. Chem. Rev. 2004, 104, 293-346.

(252) Saha, K.; Agasti, S. S.; Kim, C.; Li, X.; Rotello, V. M. Gold Nanoparticles in Chemical and Biological Sensing. Chem. Rev. 2012, 112, 2739-2779.

(253) Rosi, N. L.; Mirkin, C. A. Nanostrucutres in Biodiagnostics. Chem. Rev. 2005, 105, 1547-1562.

(254) Degliangeli, F.; Kshirsagar, P.; Brunetti, V.; Pompa, P. P.; Fiammengo, R. Absolute and Direct MicroRNA Quantification Using DNA - Gold Nanoparticle Probes. J. Am. Chem. Soc. 2014, 136, 2264-2267.

(255) Pavlov, V.; Xiao, Y.; Shlyahovsky, B.; Willner, I. Aptamer-Functionalized Au Nanoparticles for the Amplified Optical Detection of Thrombin. J. Am. Chem. Soc. 2004, 126, 11768-11769.

(256) Lee, J. S.; Ulmann, P. A.; Han, M. S.; Mirkin, C. A. A DNA-Gold NanoparticleBased Colorimetric Competition Assay for the Detection of Cysteine. Nano Lett. 2008, 8, 529-533.

(257) Frens, G. Controlled Nucleation for the Regulation of the Particle Size in Monodisperse Gold Suspensions. Nature 1973, 241, 20.

(258) Haiss, W.; Thanh, N. T. K.; Aveyard, J.; Fernig, D. G. Determination of Size and Concentration of Gold Nanoparticles from UV-Vis Spectra. Anal. Chem. 2007, 79, 4215-4221.

(259) Dulkeith, E.; Morteani, A. C.; Niedereichholz, T.; Klar, T. A.; Feldmann, J.; Levi, S. A.; Van Veggel, F. C. J. M.; Reinhoudt, D. N.; Möller, M.; Gittins, D. I. Fluorescence Quenching of Dye Molecules near Gold Nanoparticles: Radiative and Nonradiative Effects. Phys. Rev. Lett. 2002, 89, 203002. 
(260) Xu, Q.; Cao, A.; Zhang, L.; Zhang, C. Rapid and Label-Free Monitoring of Exonuclease III-Assisted Target Recycling Amplification. Anal. Chem. 2012, 84, 10845-10851.

(261) Sato, K.; Hosokawa, K.; Maeda, M. Rapid Aggregation of Gold Nanoparticles Induced by Non-Cross-Linking DNA Hybridization. J. Am. Chem. Soc. 2003, 125, 8102-8103.

(262) Herne, T. M.; Tarlov, M. J. Characterization of DNA Probes Immobilized on Gold Surfaces. J. Am. Chem. Soc. 1997, 119, 8916-8920.

(263) Levicky, R.; Herne, T. M.; Tarlov, M. J.; Satija, S. K. Using Self-Assembly To Control the Structure of DNA Monolayers on Gold: A Neutron Reflectivity Study. J. Am. Chem. Soc. 1998, 120, 9787-9792.

(264) Wu, J.; Campuzano, S.; Halford, C.; Haake, D. A.; Wang, J. Ternary Surface Monolayers for Ultrasensitive (Zeptomole) Amperometric Detection of Nucleic Acid Hybridization without Signal Amplification. Anal. Chem. 2010, 82, 88308837.

(265) Cleland, W. W. Dithiothreitol, a New Protective Reagent for SH Groups. Biochemistry 1964, 3, 480-482. 


\section{VITA}

JANAK PAUDYAL

Born, Baglung, Nepal

1999-2003

B.S., Chemistry

Birendra Multiple College

Bharatpur, Nepal

2006

M.S., Chemistry, Tribhuvan University

Kathmandu, Nepal

2011-2016

Doctoral Candidate

Florida International University

Miami, Florida

\section{PUBLICATIONS AND PRESENTATIONS}

Wang P., Paudyal J., Zhou F.Y. \& Xiao Y.* (2016) Electrocatalytic oxidation of methanol based on platinum nanoclusters decorated, carbon nanotubes functionalized paper chips. Submitted.

Paudyal J., Guntupalli B., Dai J.Y., Li W.Z. \& Xiao Y.* (2016) Disposal paper-based SWCNT thin film for electrocatalytic detection of small-molecule targets in complex matrices for disease diagnosis and drug detection. Submitted.

Paudyal J. \& Xiao Y.* (2016) A rapid and sensitive colorimetric assay based on DNAmodified gold nanoparticles for detecting DNA and exonuclease III. In preparation.

Xiao Y., Dai J.Y., Paudyal J., Guntupalli B., Liang P.P., Pubillones F., Yang Y.H., Li W.Z. \& Wang X.T. (2013) Paper-based single-walled carbon nanotube thin film for catalytic detection of nicotinamide adenine dinucleotide in human blood. Invited talk, $89^{\text {th }}$ Florida Annual Meeting and Exposition. Palm Harbor, FL, May 9 - 11. 